\author{
UNIVERSIDADE de SÃO PAULO \\ INSTITUTO de FÍSICA de SÃO CARLOS \\ DEPARTAMENTO de FÍSICA e INFORMÁTICA
}

\title{
APLICAÇÕES das TÉCNICAS de LENTE TÉRMICA e Z-SCAN ao ESTUDO de SÓLIDOS DOPADOS
}

Acácio A. de Castro Andrade

Tese apresentada ao Instituto de Física de São

Carlos, da Universidade de São Paulo, para obtenção do título de Doutor em Ciências: Física Aplicada.

Orientador: Prof. Dr. Tomaz Catunda

São Carlos 
Andrade, Acácio A. de Castro

Aplicações das técnicas de Lente Térmica e Z-scan no estudo dos sólidos dopados/ Acácio A. de Castro Andrade - São Carlos, 2002-08-16

Tese (Doutorado) - Instituto de Física de São Carlos da Universidade de São

Paulo, 2002 - páginas: 155

Área: Física Aplicada

Prof. Dr. Tomaz Catunda

1) Sólidos dopados. 2. Eficiência quântica. 3. Efeito não-linear.

I. Título 
À minha mulher, Rosimeire, que de uma maneira muito especial alegrou à minha vida.

À minha mãe, minha verdadeira mestra.

A todos os meus familiares que acreditaram na minha capacidade e vontade de vencer. 


\section{Agradecimentos}

Nenhum trabalho é feito sem auxílio. Gostaria de agradecer à $\boldsymbol{F A P E S P}$ pelo suporte financeiro e dizer um muito obrigado a um sem fim de pessoas maravilhosas.

Ao Prof. Tomaz Catunda pela orientação, apoio, incentivo e amizade durante o desenvolvimento deste trabalho de doutorado. Todo resultado obtido eu o divido com ele.

Aos meus amigos Sandro Márcio Lima e Juraci A. Sampaio, pela ajuda no laboratório e na interpretação dos resultados.

À amiga Dr. Dione F. de Souza pela leitura do meus relatórios enviados à FAPESP e pela valiosa ajuda na minha qualificação.

Aos amigos do grupo: Prof. Luiz Antonio, Carlos Jacinto, Djalmir, André, Rui, Ariane, Gláucia, Samuel, Andréia, Daniel, Alessandra, Tânia e Josimar pelos momentos de alegria e descontração.

Ao Prof. Mauro L. Baesso e ao Dr. Ronan M. Lebullenger pelas amostras de vidro aluminato de cálcio e fluoreto, respectivamente, estudadas neste trabalho.

À Ester e Gélia, secretárias do grupo, pela amizade e serviços prestados.

Às funcionárias da biblioteca do IFSC, pelo pronto atendimento, sempre com muita simpatia e eficiência.

À Wladerez A. Gounella Caiado da seção de pós-graduação, pelo pronto atendimento.

Aos funcionários da oficina mecânica do IFSC pelos serviços prestados.

À todas as pessoas, cujos nomes não constam nesta lista, mas que de uma maneira ou outra contribuíram para a realização deste trabalho. 
Índice

RESUMO ......................................................................................................................XV

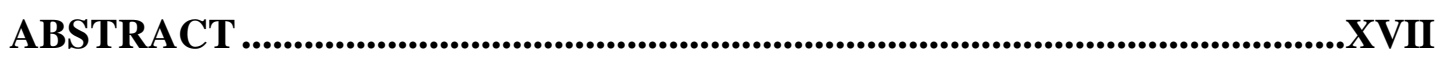

CAPÍTULO 1.............................................................................................................................1

1. INTRODUÇÃ̃

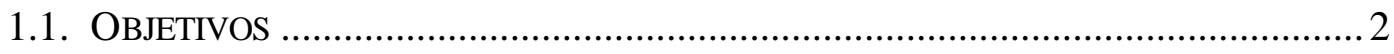

1.2. CARACTERÍSTICAS ESPECTROCóPICAS DE SÓLIDOS DOPADOS COM $N d^{3+} \ldots \ldots \ldots . .5$

1.3. INVESTIGAÇ̃̃O DA ORIGEM DA NÃO-LINEARIDADE EM SÓLIDOS DOPADOS.........8

1.4. EFEITO NÃO-LINEAR ELETRÔNICO ………………………............................ 10

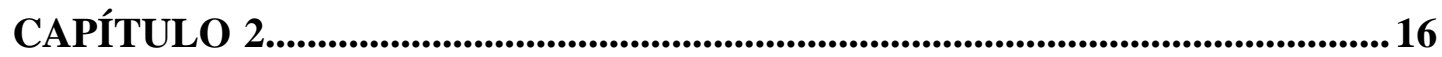

2. ESPECTROMETRIA DE LT .............................................................................. 16

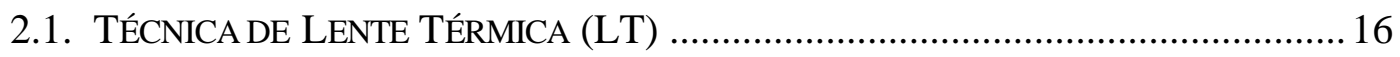

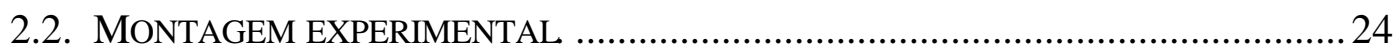

2.3. DeterminaÇão DA CINTURA DO FEIXE DO LASER. ........................................... 26

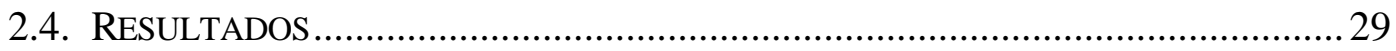

CAPÍTULO 3...........................................................................................................31

3. EFICIÊNCIA QUÂNTICA. .................................................................................31

3.1. MEdidAS de EFICIÊNCIA QuÂNTICA DA FLUORESCÊNCIA................................. 31

3.2. MEDIDAS DE EFICIÊNCIA QUÂNTICA COM A TÉCNICA DE LT............................... 40

3.3. MEDIDA DE LT EM FUNÇÃO DO COMPRIMENTO DE ONDA DE EXCTTAÇÃO..........50

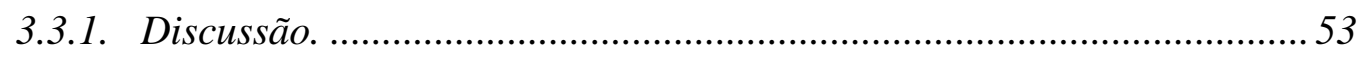

3.4. MEdIDAS DE LT NO FILME DE $P P V$ (P-FENILENO-VINILENO)...........................57

CAPÍTULO 4.................................................................................................62

4. MEDIDAS DE $d s / d T$................................................................................... 62 


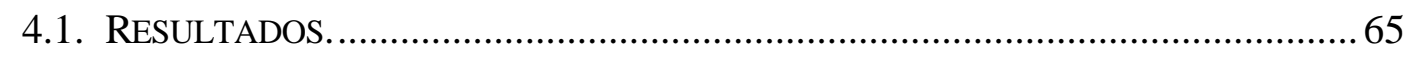

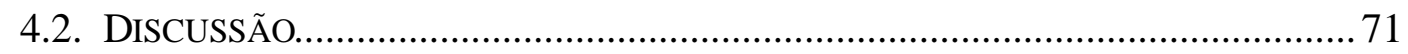

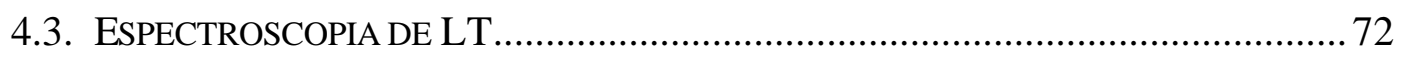

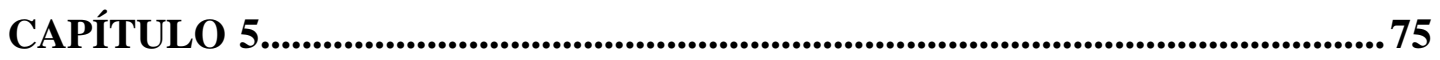

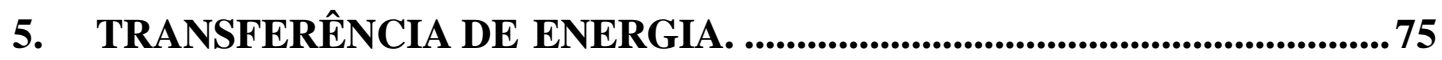

5.1. ESTUdO DOS MECANISMOS DE TRANSFERÊNCIA DE ENERGIA NOS SÓLIDOS DOPADOS COM $\mathrm{Cr}^{3+} \mathrm{E} \mathrm{Nd}^{3+}$ UTILIZANDO A TÉCNICA DE LT................................ 75

5.2. MEDidAS DE "UPCONVERSION AUGER" NO CRISTAL LiSAF DOPADO COM

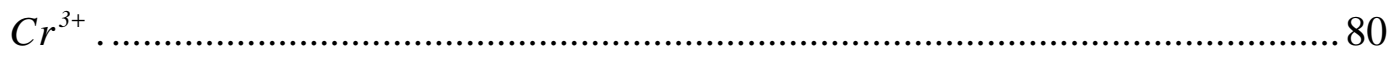

5.3. MEDIDAS DE TRANSFERÊNCIA DE ENERGIA EM SÓLIDOS DOPADOS COM $\mathrm{Nd}^{3+} \ldots$ .84

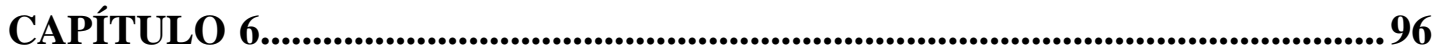

6. ESTUDO DOS EFEITOS DE LP E LT ATRAVÉS DA TÉCNICA DE Z

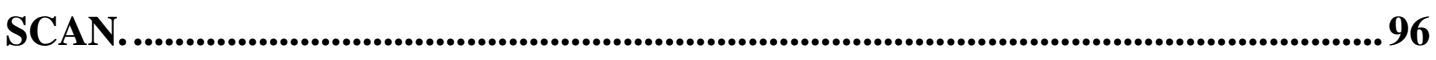

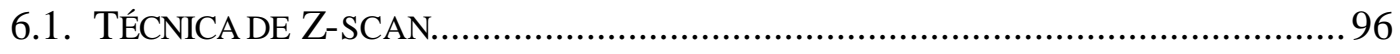

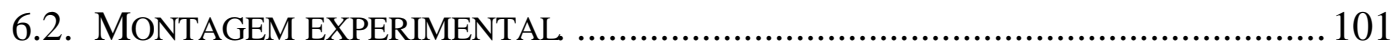

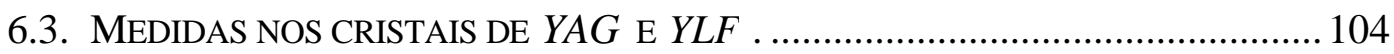

6.4. Contribuições de Lente TérmicA (LT) E Lente de PopulaçÃo (LP) EM

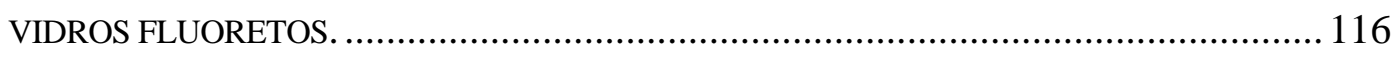

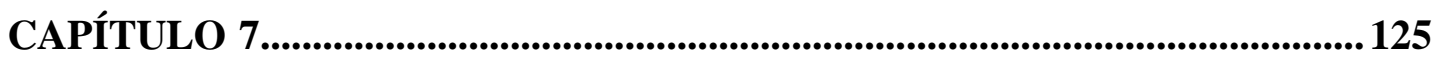

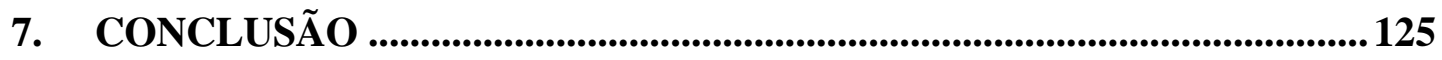

7.1. PersPeCtivas de Trabalhos FUtUROS. ..................................................... 130

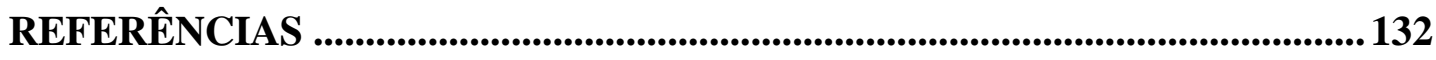

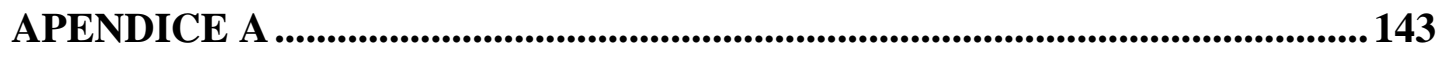

A1 TRABALHOS PUBLICADOS.................................................................... 143 


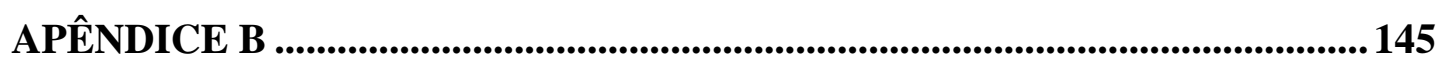

B1 AMOSTRAS ESTUDADAS ..................................................................................... 145

APÊNDICE C ................................................................................................................. 153

C1 FATOR DE CORREÇÃ 0 ................................................................................. 153 
Lista de Figuras

Figura 1.1 - Diagrama de níveis de energia do $\mathrm{Nd}^{3+}$. Figura tirada da referência [47]

Figura 1.2 - Intervalo dos tempos de vida radiativo para o estado metaestável ${ }^{4} F_{3 / 2}$ [46]

Figura 1.3 - Espectro da seção de choque de emissão da transição ${ }^{4} F_{3 / 2} \rightarrow{ }^{4} I_{11 / 2}$ para vários vidros [46]. 8

Figura 1.4 - ? a do $\mathrm{Nd}^{3+}$ em diversos materiais [4]. 10

Figura 1.5 - Diagrama de um sistema genérico de 4 níveis. 11

Figura 2.1 - Distribuição de temperatura ?T(r) na LT em função do tempo [57]. Sendo $P(\mathrm{~mW})$ a potência do laser de excitação, $A\left(\mathrm{~cm}^{-1}\right)$ o coeficiente de absorção da amostra. 17

Figura 2.2 - Arranjo dos feixes de excitação e prova na configuração do experimento de LT com feixe duplo de modo descasado, onde $L_{1}$ e $L_{2}$ são as lentes $e \mathrm{w}_{\mathrm{e}} e \mathrm{w}_{\mathrm{op}}$ os raios dos feixes de excitação e prova respectivamente... 20

Figura 2.3 - Curva da evolução temporal do efeito de LT em uma amostra de LSCAS dopada com $2 \% \mathrm{Nd}^{3+}$, com $d s / d T$ positivo. Com $A=3,3 \mathrm{~cm}^{-1} e$ $L=0,38 \mathrm{~cm}$. A medida foi realizada com $?_{\text {exc }}=810 \mathrm{~nm}$ e $P=69 \mathrm{~mW}$. Ajuste teórico realizado com a Eq. (2.3), com $m=23$ e $V=1,73$. 21

Figura $2.4-\theta$ e $t_{c}$ normalizados versus $t / t_{c}$ .22

Figura 2.5 - Erro relativo de $\theta$ e $t_{c}$ versus $t / t_{c}$ .22

Figura 2.6 - Defeito quântico. Onde g é o estado fundamental, $I_{1}$ e $I_{2}$ estados intermediários e ex o estado excitado. 24

Figura 2.7 - Aparato experimental do experimento de LT. Onde M são os espelhos, $L$ as lentes com distância focal $f \approx 20 \mathrm{~cm}, F$ os filtros, $D$ os detetores e ch o “chopper”. .25

Figura 2.8 - Quadrado do diâmetro do feixe do laser de HeNe em função da distância da lente convergente de foco, $f=20 \mathrm{~cm}$. Os dados experimentais foram ajustados com a equação da parábola. 
Figura 2.9 - Quadrado do diâmetro do feixe de prova (HeNe) em 632nm, com o centro da parábola em $z=0$. Os dados experimentais foram ajustados com a equação da parábola. .28

Figura 2.10 - Sinal do feixe de prova, $?_{p}=632 \mathrm{~nm}$, com $P=54 \mathrm{~mW} \lambda_{\text {exc }}=808 \mathrm{~nm}$. Ajuste teórico Eq. (2.3), com $V=1,73$ e $m=16,4$.

Figura 3.1 - Processos de “quenching” da fluorescência para o $\mathrm{Nd}^{3+}$. Figura tirada da Ref. [68].

Figura 3.2 - Tempo de vida em função da concentração. Figura tirada da Ref. [68].

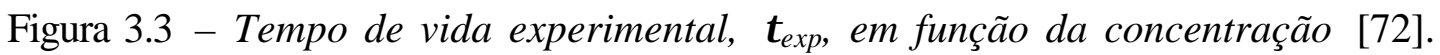
Ajuste teórico usando a expressão (3.3). 36

Figura 3.4 - Eficiência quântica do vidro silicato ED-2:Nd ${ }^{3+}$ em função da concentração. Os dados de (a) foram obtidos por Rosencwaig [66] por método fotoacústico, (b) pelo método de Judd-Ofelt por Linford e colaboradores [83] com $t_{R}=359 \mu \mathrm{s}$ e (c) pelo método de Judd-Ofelt por Krupke [84] com $t_{R}=372 \mu s$.

Figura 3.5 - Eficiência quântica do vidro fosfato LG-750:Nd ${ }^{3+}$ em função da concentração. Os resultados de (a) e (b) foram determinados por Caird e colaboradores [85] utilizando o método da esfera integradora e o método de Judd-Ofelt com $t_{R}=352 \mu \mathrm{s}$, respectivamente. $O$ resultado indicado por (c) foi obtido por Ramponi [86] utilizando o método de calorimetria.

Figura 3.6 - Eficiência quântica do YAG:Nd ${ }^{3+}$ em função da concentração obtida por vários métodos. (a) Krupke [79] com $t_{R}=259 \mu \mathrm{s}$; (b) Powell $e$ colaboradores [87], (c) Singh e colaboradores [88] e (d) Rosencwaig e Edward [66] 40

Figura 3.7 - Diagrama parcial dos níveis de energia do $\mathrm{Nd}^{3+}$ 41

Figura 3.8 - Sinal de lente térmica do vidro aluminato de cálcio (LSCAS), para diferentes valores da potência de excitação do laser em $810 \mathrm{~nm}$. Comprimento de onda do feixe de prova $?_{p}=632 \mathrm{~nm}$. A linha sólida é o ajuste linear. 
Figura 3.9 - Sinal de lente térmica normalizado versus a concentração de $\mathrm{Nd}^{3+}$ para $o$ vidro aluminato $\left(\right.$ LSCAS) em $?_{\mathrm{exc}}=810 \mathrm{~nm}$

Figura 3.10 - Eficiência quântica do vidro aluminato (LSCAS) em função da concentração de $\mathrm{Nd}^{3+}$. (a) Resultado de LT em $?_{\text {exc }}=810 \mathrm{~nm} ;$ (b) resultado em $?_{\text {exc }}=514 \mathrm{~nm} ;(\mathrm{c})$ resultado obtido pelo método de Judd-Ofelt e (d) ajuste teórico usando a Eq. (3.3) obtendo $N_{c q}=(5,48 \pm 0,05) \times 10^{20}$ ions $/ \mathrm{cm}^{3}, p=2 e$ $t_{0}=(322 \pm 2) \mu s$ .44

Figura 3.11 - Eficiência quântica do vidro fluoreto ZBLAN. ? ${ }_{\text {exc }}=797 \mathrm{~nm}$. $N_{c q}=(5,3 \pm 0,2) \times 10^{20}$ ions $/ \mathrm{cm}^{3}, p=2$ e $t_{0}=(527 \pm 11) \mu \mathrm{s}$ 46

Figura 3.12 - Eficiência quântica do vidro fluoreto PGIZCa. $\lambda_{\text {exc }}=797 \mathrm{~nm}$.

$$
N_{c q}=(4,8 \pm 0,2) \times 10^{20} \text { ions } / \mathrm{cm}^{3}, p=2 \text { e } t_{0}=(446 \pm 8) \mu \mathrm{s}
$$

Figura 3.13 - Eficiência quântica, $\eta$, versus a concentração de $\mathrm{Nd}^{3+}$ para $Y A B C$, ZBLAN, PGIZCa LSCAS e GaLaS .48

Figura 3.14 - Sinal de lente térmica normalizado versus o comprimento de onda de excitação no aluminato ( LSCAS) com 2,0\% $\mathrm{Nd}^{3+}$. Comprimento de onda de prova $?_{p}=632 \mathrm{~nm}$ .51

Figura 3.15 - Sinal de lente térmica normalizado versus o comprimento de onda de excitação, no fluorozirconato (ZBLAN) com $0,5 \% \mathrm{Nd}^{3+}$. Comprimento de onda de prova $?_{p}=632 \mathrm{~nm}$. .52

Figura 3.16 - Medidas de lente térmica em função do comprimento de onda no YAG. Espessura da amostra $L=2,1 \mathrm{~mm}$ e concentração de $0,75 \%$ de $\mathrm{Nd}^{3+}$. Comprimento de onda de prova $?_{p}=632 \mathrm{~nm}$ e $\left\langle ?_{\text {em }}^{-1}\right\rangle=0,952 \mu \mathrm{m}^{-1}$

Figura 3.17 - Comportamento do inverso da eficiência quântica em função da concentração de $\mathrm{Nd}^{3+}$ para os vidros: LSCAS, ZBLAN e PGIZCa. Gráficos feitos com os dados da Tabela 3.4. A linha sólida representa o ajuste feito com a Eq. (3.7). .54 
Figura 3.18 - O símbolo $(o)$ representa o gráfico de $\left(t_{0} / t_{\text {exp }}\right)-1$ e $(\nabla)$ o gráfico de $\left(?_{0} / ?_{L T}\right)-1$, ambos em função da concentração de $\mathrm{Nd}^{3+}$. A linha sólida é o ajuste linear dos dados. .55

Figura 3.19 - O símbolo $(o)$ representa o gráfico de $\left(t_{0} / t_{\text {exp }}\right)-1$ e $(\nabla)$ o gráfico de $\left(?_{0} /\right.$ ?) - 1. Os dados de (a) foram tirados da Ref. [43]; (b) da Ref. [85]; (c) da Ref. [72] e (d) da Ref. [66].. .56

Figura 3.20 - Espectro de absorção ótica e fotoluminescência $\left(T=300^{\circ} \mathrm{K}\right)$ do filme PPV tratado a $110^{\circ} \mathrm{C}$. As medidas foram realizadas pelo Dr. A. Marlleta no grupo de polímeros do IFSC. .58

Figura 3.21 - Espectro de excitação da fotoluminescência (PLE). .59

Figura 3.22 - Sinal da fotolumenescência (linha forte) e intensidade da luminescência (o). Ambos os sinais foram normalizados pela absorbância (linha fraca) e pelo comprimento de onda de excitação. Os resultados estão apresentados mesma escala.

Figura 3.23 - Sinal de lente térmica normalizado versus o comprimento de onda de excitação para o PPV. Comprimento de onda de prova $?_{p}=632 \mathrm{~nm} e$ $\left\langle ?_{\text {em }}^{-1}\right\rangle=1,81 \mu m^{-1}$

Figura 4.1 - Diagrama esquemático da variação do caminho ?l(r) induzida pelo laser.

Figura 4.2 - Orientação das amostras investigadas em relação a polarização do feixe de prova. Onde $\hat{k}$ é o vetor de onda.

Figura 4.3 - Sinal de LT no cristal LiSAF com $1 \%$ de $\mathrm{Cr}^{3+}$. Feixe de excitação $\left(\lambda_{\text {exc }}=488 \mathrm{~nm}\right)$ com polarização $E \perp c$. (o) feixe de prova $\left(?_{p}=632 \mathrm{~nm}\right)$ polarizado de acordo com configuração $(a)$ e $(\cap)$ configuração $(b)$ da Figura 4.2, respectivamente. Ajuste teórico feito com a Eq. (2.3). Com $w=4,15 \times 10^{-3} \mathrm{~cm}^{2}, \quad V=1,73, \quad m=19,2$ e $t_{c}=(0,58 \pm 0,01) \times 10^{-3} \mathrm{~s}$ para ambas as curvas. 68

Figura 4.4 - Medidas de LT no cristal LiSAF com o feixe de prova $\left(?_{p}=632 \mathrm{~nm}\right)$ polarizado. (ロ) representa a medida feita com o laser de prova com 
polarização paralela ao eixo c da amostra e (o) com polarização perpendicular ao eixo c.

Figura 4.5 - Sinal de LT no cristal LiSAF com $1 \%$ de $\mathrm{Cr}^{3+}$. Feixe de excitação $\left(\lambda_{\text {exc }}=488 \mathrm{~nm}\right) \mathrm{com}$ polarização $E \perp c$ e feixe de prova $\left(?_{p}=632 \mathrm{~nm}\right)$ polarizado de acordo com a configuração (a). Ajuste teórico feito com a Eq.

$$
w_{e}=4,15 \times 10^{-3} \mathrm{~cm}^{2}, \quad t_{c}=(0,70 \pm 0,01) \times 10^{-3} \mathrm{~s}
$$

$$
?=(11,23 \pm 0,02) \times 10^{-2}
$$

Figura 4.6 - Espectro de transmitância do vidro calcohaleto no infravermelho próximo. .73

Figura 4.7 - Variação do caminho ótico com o calor depositado por unidade de volume, ds/dQ, (círculo aberto) e coeficiente de absorção, A, (círculo fechado) versus o comprimento de onda, $\lambda$.

Figura 5.1 - Diagrama esquemático dos processos de transferência de energia no $\mathrm{Nd}^{3+}$ (a) "Upconversion Auger" (b) Relaxação cruzada. Figura tirada da Ref. [49]. .76

Figura 5.2 - Simulação da fração de população do estado excitado em função do parâmetro do saturação $S$, para vários valores do parâmetro de perda $\beta$....79

Figura 5.3 - Simulações teórica do sinal de LT normalizado versus o parâmetro de saturação $\mathrm{S}$ para vários valores de $\mathrm{B}$ onde $T=$ ?/PAL $L_{\text {eff }}$. Para estas simulações foram adotados $\left(1 / k ?_{p}\right) d s / d T=1 \quad e ?_{e x c}\left\langle ?_{e m}^{-1}\right\rangle=0,5$

Figura 5.4 - Níveis de energia do $\mathrm{Cr}^{3+}$. Sendo $h ?_{\text {exc }}$ a energia do fóton de excitação e $h ?_{\text {em }}$ a energia do fóton emitido.

Figura 5.5 - Espectro de absorção do LiSAF com $1 \% \mathrm{Cr}^{3+}$. O espectro mostra a absorção nas direções paralela e perpendicular ao eixo c. $L=3,2 \mathrm{~mm}$.

Figura 5.6 - Sinal de LT para diferentes potências do feixe de excitação em $\lambda_{\text {exc }}=488 \mathrm{~nm}$, para o LiSAF com $7 \%\left(L=1,4 \mathrm{~mm}\right.$ e $\left.A=3,37 \mathrm{~cm}^{-1}\right)$ e com $1 \%\left(L=2,4 m m\right.$ e $\left.A=0,66 \mathrm{~cm}^{-1}\right)$.

Figura 5.7 - (a) $\Theta$ em função do parâmetro de saturação $S_{0}$. Os valores de $T_{0}$ são $0,31 \pm 0,01$ e 0,44 \pm 0,01 para 1 e 7\%, respectivamente. Ajuste realizado com a Eq.(5.10). (b) Eficiência quântica em função de $S_{0}$ 
Figura 5.8 - Sinal de LT normalizado por $L_{\text {eff }}$ em função da potência do feixe de excitação em $?_{\text {exc }}=797 \mathrm{~nm}$, no vidro fluorozirconato dopado com $\mathrm{Nd}^{3+}$ (ZBLAN).

Figura 5.9 - $T$ em função do parâmetro de saturação $S_{0}$. Ajuste realizado com a Eq.(5.10). 85

Figura 5.10 - Comportamento da eficiência quântica da fluorescência em função do parâmetro $S_{0}$ .86

Figura 5.11 - Valores de ? ${ }_{u p}$ do fluorozirconato (ZBLAN) em função da concentração de $\mathrm{Nd}^{3+}$ 87

Figura 5.12 - Sinal de LT normalizado por $L_{\text {eff }}$ em função da potência do feixe de excitação em $?_{\mathrm{exc}}=797 \mathrm{~nm}$, no vidro fluoroindato $(P G I Z C a)$ dopado com $\mathrm{Nd}^{3+}$. 90

Figura 5.13 - $T$ em função do parâmetro de saturação $S_{0}$. Ajuste realizado com a Eq.(5.10). 90

Figura 5.14 - Eficiência quântica da fluorescência em função do parâmetro $S_{0} \ldots . .91$

Figura 5.15 - Valores de $?_{u p}$ em função $N_{o}$ para o PGIZCa .91

Figura 5.16 - Sinal de LT normalizado por $A L_{\text {eff }}$ em função da potência do feixe de excitação em $\lambda_{\text {exc }}=514 \mathrm{~nm}$. .93

Figura 5.17 - T em função do parâmetro de saturação $S_{0}$, com $\lambda_{\text {exc }}=514 \mathrm{~nm} \ldots . . .93$

Figura 5.18 - Sinal de LT normalizado por $A L_{\text {eff }}$ em função da potência do feixe de excitação em $\lambda_{\text {exc }}=869 \mathrm{~nm}$. .94

Figura 5.19 - T em função do parâmetro de saturação $S_{0}$, com $\lambda_{\text {exc }}=869 n$ m...... 94 Figura 5.20 - Sinal de LT normalizado por $A L_{\text {eff }}$ em função da potência do feixe de excitação em $\lambda_{\text {exc }}=808 \mathrm{~nm}$ .95

Figura 5.21 - T em função do parâmetro de saturação $S_{0}$, com $\lambda_{\text {exc }}=808 \mathrm{~nm} \ldots . . . .95$ Figura 6.1 - Efeito de lente na técnica de Z-scan para o caso em que $n_{2}>0$. Em (a) temos o caso em que a amostra está situada antes do foco $(z<0)$ e o feixe aparece expandido na posição da íris. Em (b) a amostra se encontra após o foco $(z<0)$ e o feixe tende a ser colimado. Em (c) temos a curva característica 
de Z-scan, com $n_{2}>0$. A linha pontilhada representa o feixe quando o efeito não-linear é nulo $\left(n_{2}=0\right)$. .98

Figura 6.2 - Perfil do índice de refração $n(r)$ num meio não-linear saturável para $S=0$ e $S=10$. 101

Figura 6.3 - Aparato experimental utilizado nas medidas de Zscan. L representa lentes convergentes, $E$ espelhos, $D$ detetores, $D v$ divisor de feixe e Ch “chopper”. Os sistema de aquisição é constituído por um microcomputador (M), um osciloscópio (OS) e um amplificador (AM). 103

Figura 6.4 - Evolução temporal do sinal de Zscan da amostra de $\mathrm{GdAlO}_{3}: \mathrm{Cr}^{3+}$ $\left(n_{2}>0\right)$, colocada em (a) $z=0.85 z_{c}$, (b) $z=-0.85 z_{c}$ e (c) $z=7 z_{c}[11] \ldots \ldots 103$

Figura 6.5 - Medida de Zscan resolvido no tempo no cristal de YAG:Nd ${ }^{3+}$, com 0,44\% de $\mathrm{Nd}^{3+}(L=1,4 \mathrm{~mm})$. Freqüência do chopper $(f=820 \mathrm{~Hz}), \lambda_{\text {exc }}=514 \mathrm{~nm}$ e potência $(P=190 \mathrm{~mW})$. Ajuste teórico feito com a expressão de Z-scan , Eq. (6.2) [107]. $A_{b 1}$ e $A_{b 2}$ representam a abertura da fenda. 105

Figura 6.6 - Medida de Z-scan resolvido no tempo no cristal de YAG:Nd ${ }^{3+}(0,44 \%$ de $\mathrm{Nd}^{3+}$ e $\left.L=0,14 \mathrm{~cm}\right)$, com $\lambda=808 \mathrm{~nm}, P=102 \mathrm{~mW}\left(I_{0}=7,2 \mathrm{KW} / \mathrm{cm}^{2}\right) e$ $f=820 \mathrm{~Hz}$. (a) fenda fechada $A_{b 1}=50 \%$ (ajuste teórico realizado com a Eq. (6.2)); (b) fenda aberta $A_{b 2}=100 \%$ (ajuste realizado com a Eq. (6.5)) [107]. 106

Figura 6.7 - Variação de ? $T_{\mathrm{pv}}\left(Y A G\right.$ com $0,75 \%$ de $\left.\mathrm{Nd}^{3+}\right)$ em função da intensidade, $I_{0}$, com $A_{b 1}=50 \%$ e $\lambda=808 \mathrm{~nm}$. 108

Figura 6.8 - Simulações teóricas de ? T versus $S_{0}$. (a) $\beta=0$ com a Eq. (6.16), (b) $\beta=5$ com a Eq. (6.18) integrada e (c) $\beta=5$ com a Eq. (6.18) sem integrar. 110

Figura 6.9 - Variação da amplitude do sinal da fenda aberta em função do parâmetro de saturação $S_{0}$. YAG com $0,75 \%$ de $\mathrm{Nd}^{3+}$. Ajuste realizado com a Eq. (6.18) usando $I_{s}=17,6 \mathrm{KW} / \mathrm{cm}^{2}$. Do ajuste obtivemos $\beta=5,7 \pm 1,1 \mathrm{e}$ $? \mathrm{~s}=-(4,5 \pm 2) \times 10^{-20} \mathrm{~cm}^{2}$. 
Figura 6.10 - (a) Dependência temporal do sinal de Zscan no YAG excitando em $\lambda=808 n m .(b)$ - valores de $\tau^{-1}$ versus $I_{p}$

Figura 6.11 - Z-scan resolvido no tempo para o YLF . (a) fenda fechada $A_{b 1}=50 \%$, (b) fenda fechada $A_{b 2}=100 \%, f=820 \mathrm{~Hz}, P=250 \mathrm{~mW}$, linha contínua ajuste teórico feito com as expressões de Z-scan. 114

Figura 6.12 - Variação de T $_{\mathrm{pv}}$ do cristal YLF em função da intensidade, $I_{0}$, com $A_{b 1}=50 \%$ e $\lambda=802 \mathrm{~nm}$. A linha sólida representa o ajuste feito com a Eq.(6.18).

Figura 6.13 - Variação da amplitude do sinal da fenda aberta em função do parâmetro de saturação $S_{0}$, para o cristal YLF. Ajuste com a Eq.(6.18). $\beta=15,7 \pm 4,6$

Figura 6.14 - Medida de Z-scan resolvido no tempo com abertura, $A_{b 2}=100 \%$, para o InSBZnGdN dopado com $N d^{3+}$, com $f=824 \mathrm{~Hz}, \quad P=187 \mathrm{~mW} \quad e$ $\lambda=514 \mathrm{~nm}$. A linha cheia indica o ajuste feito com a Eq. (6.8).

Figura 6.15 - Z-scan resolvido no tempo com $\lambda=514 \mathrm{~nm}$ e abertura $A_{b 1}=50 \%$, para o InSBZnGdN dopado com $N d^{3+}$. (•?) freqüência do "chopper" $f=180 \mathrm{~Hz}, P=230 \mathrm{~mW}$, (o) $f=824 \mathrm{~Hz}$ e $P=187 \mathrm{~mW}$

Figura 6.16 - Z-scan resolvido no tempo com $\lambda=514 \mathrm{~nm}$ e abertura $A_{b 1}=50 \%$, para o vidro ZBLAN dopado com $\mathrm{Nd}^{3+}$. (O) freqüência do "chopper" $f=150 \mathrm{~Hz}, P=145 \mathrm{~mW},(\bullet \rho)=824 \mathrm{~Hz}$ e $P=245 \mathrm{~mW}$.

Figura 6.17 - Z-scan resolvido no tempo com $\lambda=514 \mathrm{~nm}$ e abertura $A_{b 1}=50 \%$, para o InSBZnGdN dopado com CoF . $_{2}$ (•) Freqüência do "chopper" $f=824 \mathrm{~Hz}, P=100 \mathrm{~mW}$, (o) $f=10 \mathrm{~Hz}$ e $P=100 \mathrm{~mW}$.

Figura B 1 - Espectro de absorção dos vidros fluorozirconato (ZBLAN) e aluminato (LSCAS). 147

Figura B 2- Espectro de absorção do YAG: $\mathrm{Nd}^{3+}$, amostra (1). Espessura da amostra $L=1,4 \mathrm{~mm}$. 
Figura B 3- Espectro de absorção do $Y A G: N d^{3+}$, amostra (2). Espessura da amostra $L=2,1 \mathrm{~mm}$.

Figura C 1 - A Figura (a) e (c) onde há a deformação na forma do sinal. A Figura (b) e (d) apresenta um caso ideal, sem deformação do sinal. 153

Figura C 2 - Forma do sinal com uma deformação devido ao tempo de abertura do chopper. 155 
Lista de Tabelas

Tabela 2.1 - Valores típicos dos parâmetros usados nos experimentos de LT.......... 28 Tabela 2.2 - Valores de difusividade térmica $(D)$. Sendo $\rho$ a densidade, c o calor específico, $k_{\text {Cal }}$ a condutividade térmica calculada, $k_{\text {Lit }}$ a condutividade térmica da literatura. (a) [61], (b) [62], (c) [7], (d) [16], (e) [6, 13], (f) [31]. 30

Tabela 3.1 - Valores da concentração de “quenching” para o $\mathrm{Nd}^{3+}$ 35

Tabela 3.2 - Tabela comparando a concentração cúbica de supressão $\left(N_{c q}\right), ?_{J O}$ eficiência quânticao, tempo de vida de baixa concentração $t_{0}$ (obtido através da Eq. (3.3)) e o tempo de vida radiativo, $t_{R}$, calculado pelo método de JO. .. 37

Tabela 3.3 - Tabela comparando o tempo de vida de baixa concentração $t_{0}$ (obtido com a Eq. (3.3)) e o tempo de vida radiativo, $t_{R}$, calculado pelo método de JO. $?_{\text {Jo }}$ obtido pelo método de Judd-Ofelt e ? ${ }_{0}$ obtido por LT. (a) Ref. [71]; (b) Ref. [72]; (c) Ref. [89].

Tabela 3.4 - Parâmetros termo óptico experimental dos vidros: Aluminato (LSCAS) com os resultados em $\lambda_{\text {exc }}=810 \mathrm{~nm}$; Fluoraluminato (YABC), Fluoroindato $(P G I Z C a)$ e Fluorozirconato (ZBLAN) com resultados obtidos em $\lambda_{\text {exc }}=797 \mathrm{~nm} . t_{\text {exp }}$ representa o valor experimental do tempo de vida. Nos fluoretos $\left\langle ?_{e m}^{-1}\right\rangle=0,97 \mu m^{-1}$ [72]. (a) Dados obtidos da Ref. [7].

Tabela 3.5 - Tabela com os parâmetros obtidos através das medidas de LT e do tempo de vida. (a) Dados tirados da Ref. [72], (b) dado tirado da Ref. [30]; (c) obtido por esfera integradora [85]; (d) obtido por fotoacústica [66].....

Tabela 3.6 - Eficiência quântica do PPV determinada pelo método de lente térmica.

Tabela 4.1 - Propriedade das amostras estudados. (b) Ref. [93]; (c) Ref. [94]; (a) Ref. [51]. $?_{a}=(n-1)(1+\mu) a e ?_{q}=n^{3} Y \alpha\left(q_{\perp}+q_{/ /}\right) / 4$

Tabela 4.2 - Propriedade das amostras estudados. (a) Ref. [96]; (b) Ref. [61]; (c) dados do LiSAF ; (d) $\Delta_{q}$ calculado com os dados do YAG; (e) calculado com os dados do $\mathrm{CaF}_{2} . ?_{a}=(n-1)(1+\mu) a e ?_{q}=n^{3} Y \alpha\left(q_{\perp}+q_{/ /}\right) / 4 \ldots \ldots \ldots \ldots \ldots \ldots . . . .70$

Tabela 5.1 - Resultados obtidos de ? ${ }_{\text {up }}$ para o LiSAF. .84 
Tabela 5.2 - Resultados do processo de transferência de energia em função da concentração de $\mathrm{Nd}^{3+}$ no vidro fluorozirconato (ZBLAN). 86

Tabela 5.3 - Resultados do processo de transferência de energia, em função da concentração de $\mathrm{Nd}^{3+}$ no vidro fluoroindato ( PGIZCa) e no cristal YLF .....91

Tabela 6.1 - Valores dos parâmetros obtidos no YAG. (a) valor obtido da Ref. [108]. $O$ valor de $I_{s}$ foi obtido através da relação $I_{s}=h ? /$ ? st . 106

Tabela 6.2 - Tempo de vida da fluorescência, $\tau_{0}$, e intensidade de saturação, $I_{s}$ do $Y A G$ 113

Tabela 6.3 - Resultados do parâmetro de transferência de energia $\gamma$. 116

Tabela 6.4 - Principais parâmetros e resultados de Z-scan em vidros fluoretos dopados com $\mathrm{Nd}^{3+} \lambda=514 \mathrm{~nm}$.

Tabela B 1- Tabela com a composição dos vidros estudados neste trabalho. Para o aluminato $x=0,5 ; 1,0 ; 2,0 ; 2,5 ; 3,0 ; 4,0$ e 4,5 mol \%. Para o ZBLAN $x=0,5$; 1,0; 2,0 e 3,0 mol\%. Para o PGIZCa $x=0,5 ; 1,0 ; 2,0$, e 3,0 mol \%. Para o YABC $x=1,0$ e 2,0 $\mathrm{mol} \%$.

Tabela B 2 - Valores do coeficiente de absorção para o YAG em 514 e 808nm.. 151

Tabela B 3 - Tabela com os valores de absorção e de seção de choque encontrados na literatura para amostras de YAG com diferentes concentrações e nos comprimentos de onda 514 e $808 \mathrm{~nm}$..... 152 


\section{Resumo}

Neste trabalho utilizamos como ferramentas de estudo as técnicas de Lente Térmica (LT) e Z-scan, ambas resolvidas no tempo, para caracterizar parâmetros térmicos e eletrônicos do índice de refração não-linear de sólidos dopados.

O método de LT usando uma amostra referência e o método variando o comprimento de onda de excitação, foram usados para determinar a eficiência quântica da fluorescência de sólidos dopados com $\mathrm{Nd}^{3+}$. $\mathrm{O}$ efeito da concentração e os processos de transferência de energia por "upconversion Auger" e relaxação cruzada também foram estudados neste trabalho.

A técnica de LT também foi aplicada para determinar o coeficiente de caminho ótico com a temperatura em vários materiais. Foram estudados vidros fluoretos, vidros aluminato de cálcio com baixa concentração de sílica e os cristais YAG, $\mathrm{YVO}_{4}, \mathrm{YLF}, \mathrm{LiSAF}$ e LiSGaF. Os resultados mostram o potencial da técnica de LT para obter o valor absoluto deste importante parâmetro ótico, o qual é crucial para quem trabalha no projeto de cavidade de laseres de estado sólido.

Em alguns sólidos dopados com íons terras-rara, existe um índice de refração não linear $\mathrm{n}_{2}$ devido a diferença de polarizabilidade, ? a, entre os estados fundamental e excitados, chamado efeito de Lente de População (LP). Geralmente, parte da energia absorvida é convertida em calor, gerando o efeito chamado de Lente Térmica (LT). Neste trabalho mostramos que a técnica de Z-scan pode, em alguns casos, discriminar as não linearidades eletrônica (LP) e térmica (LT). Nós medimos $\mathrm{n}_{2}$ em vários vidros fluoretos dopados com $\mathrm{Nd}^{3+}$. A diferença de polarizabilidade, ?a, e a diferença de seção de choque de absorção, ? $\sigma$, entre o estado excitado 
$\left({ }^{4} \mathrm{~F}_{3 / 2}\right)$ e o estado fundamental $\left({ }^{4} \mathrm{I}_{9 / 2}\right)$ foram determinados em fluorozirconato, fluoroindato e fluoroaluminato. 


\section{Abstract}

In this work we have associated the time-resolved Thermal Lens (TL) and Z scan techniques to characterize the thermal and electronic parameters of solids.

The TL method, using a reference sample, and the multiwavelength method were used to determine the fluorescence quantum efficiency of $\mathrm{Nd}^{3+}$-doped solids. The concentration doping effects and energy transfer process via Auger upconversion, as well as the cross relaxation were also studied in this work.

The TL technique was also applied to determine the thermal optical path length coefficient in several materials. The sample studied were fluoride and low silica-content calcium aluminate glasses, and the crystals YAG, YVO ${ }_{4}, \mathrm{YLF}$, LiSAF and LiSGaF. Results indicate the potentiality of the TL to obtain the absolute value of this important optical parameter, which is of crucial importance to those working with solid state laser cavities.

In some rare-earth ion-doped solids there is a non-linear refractive index $n_{2}$ due to the polarizability difference, $\Delta \alpha$, between excited and ground states, the socalled Population Lens (PL) effect. Usually, part of the absorbed energy is converted into heat originating the so-called Thermal Lens (TL) effect. In this work, it is also shown that the Z-scan technique may, in some cases, discriminate the electronic (PL) and thermal (TL) non-liearities. We measured the $\mathrm{n}_{2}$ in several $\mathrm{Nd}^{3+}$ doped fluoride glasses. The polarizability difference, $\Delta \alpha$, and absorption cross-section difference, $\Delta \sigma$, between excited $\left({ }^{4} \mathrm{~F}_{3 / 2}\right)$ and ground $\left({ }^{4} \mathrm{I}_{9 / 2}\right)$ states of $\mathrm{Nd}^{3+}$ were determined in fluorozirconate, fluoroindate and fluoroaluminate glasses. 


\section{Capítulo 1}

\section{Introdução}

As propriedades não-lineares de sólidos dopados com íons têm sido estudadas por diferentes técnicas: medidas interferométricas [1], misturas de ondas [2-4], automodulação espacial de fase, lente térmica [5-7] e Z-scan [5, 8-12]. Os sólidos dopados com $\mathrm{Nd}^{3+}$ e $\mathrm{Cr}^{3+}$ têm sido bastante estudados devido suas aplicações em laseres. Nestes materiais as propriedades não lineares ocorrem devido à população do estado excitado (metaestável) de íons dopantes, a qual tem uma susceptibilidade diferente do estado fundamental. Denominamos este efeito de lente de população (LP), o qual gera um índice de refração não linear complexo. As partes real $\left(n_{2}\right)^{\prime}$ e imaginária $\left(\mathrm{n}_{2}^{\prime \prime}\right)$ do índice de refração $\mathrm{n}_{2}$ são proporcionais à polarizabilidade eletrônica ?a e a seção de choque de absorção, ?s entre os estados excitados e o estado fundamental, respectivamente. Na maior parte dos sólidos dopados com $\mathrm{Cr}^{3+}$ ou $\mathrm{Nd}^{3+}, \mathrm{n}_{2}$ é uma ordem de magnitude maior do que $\mathrm{n}_{2}$. Geralmente, parte da população do estado excitado decai via um processo não radiativo, de maneira que o material é aquecido, gerando uma variação no caminho óptico devido ao coeficiente de temperatura do caminho óptico, ds/dT . Este efeito chamamos de efeito de Lente Térmica (LT).

Uma das mais importantes propriedades de materiais óticos fluorescentes é a eficiência quântica, ?, definida pela razão entre o número de fótons absorvidos e emitidos. É bem conhecido que para obter laseres de estado sólido eficientes, o meio ativo, geralmente constituído de vidros ou cristais dopados com íons, tem que 
apresentar alta taxa de emissão de radiação. Além disso, para outras aplicações, tal como amplificadores óticos, o valor de ? precisa ser próximo da unidade. No entanto, a determinação do valor absoluto de ? para materiais luminescentes é muito importante e indispensável para várias aplicações.

Apesar do grande número de trabalhos realizados nos últimos anos, ainda existem muitos resultados controversos apresentados na literatura, principalmente sobre eficiência quântica. De modo que, estudos cada vez mais aprofundados, são necessários para o conhecimento destas propriedades, térmicas e eletrônicas, cuja importância é fundamental para quem trabalha no projeto de cavidades de laseres de estado sólido. De modo geral, este trabalho contribui para um melhor entendimento destas propriedades em sólidos dopados, utilizados como meio ativo para laseres.

\subsection{Objetivos}

Este trabalho tem como objetivos apresentar os resultados de:

$\checkmark$ Difusividade térmica;

$\checkmark$ Eficiência quântica em sólidos dopados com diferentes concentrações de $\mathrm{Nd}^{3+}$

$\checkmark$ Variação do caminho ótico com a temperatura (ds/dT );

$\checkmark$ Processos de transferência de energia (por "upconversion Auger" e relaxação cruzada);

$\checkmark$ Estudo da separação entre as propriedades não lineares eletrônica e térmica.

Para isto, nós fizemos uso de duas importantes técnicas: a de Lente Térmica (LT) [13] e a de Z-scan [14], ambas resolvidas no tempo. A idéia básica destas 
técnicas consiste em relacionar a medida da variação da intensidade no centro do feixe no campo distante com a variação do índice de refração da amostra. Estas técnicas se destacam por sua simplicidade na montagem experimental, pela alta sensibilidade e por serem técnicas não contaminantes e não destrutivas.

O presente trabalho está estruturado da seguinte maneira:

$\checkmark$ Capítulo 1 - Introdução. Neste capítulo é feito uma breve revisão das características de sólidos dopados com $\mathrm{Nd}^{3+}$ e uma revisão sobre a origem da não linearidade eletrônica em sólidos dopados.

$\checkmark$ Capítulo 2 - Espectrometria de LT. Neste capítulo é apresentado o efeito de LT, a configuração experimental utilizada e os resultados das propriedades térmicas em sólidos, difusividade térmica e condutividade térmica.

$\checkmark$ Capítulo 3 - Medidas de eficiência quântica. Neste capítulo apresentamos um estudo sobre o decréscimo da eficiência quântica com a concentração de $\mathrm{Nd}^{3+}$ e comparamos os nossos resultados de LT com outras técnicas.

$\checkmark$ Capítulo 4 - Medidas de ds/dT. Apresentamos um estudo pioneiro de comparação de valores de ds/dT experimentais, obtidos através da técnica de LT, com o teórico que considera a contribuição de $d n / d T$, coeficiente de expansão térmica e do efeito fotoelástico.

$\checkmark$ Capítulo 5 - Transferencia de energia. Neste capítulo mostramos que a técnica de LT também pode ser utilizada como uma ferramenta aternativa para determinar os processos de transferência de energia, que pode ser por "upconversion Auger" ou relaxação cruzada. Estes processos são responsáveis pela diminuição do valor absoluto da eficiência quântica 
com o aumento da concentração de dopante. Nós estudamos conjuntos de amostras com diferentes concentrações. Apesar de existir vários trabalhos a respeito deste assunto, são poucos os trabalhos que retratam estes processos para um mesmo conjunto de amostra com diferentes concentrações.

$\checkmark$ Capítulo 6 - Estudo dos efeitos de LP e LT através da técnica de Z-scan. Este capítulo é dedicado ao estudo da separação entre as propriedades não lineares eletrônica e térmica, através da técnica de Z-scan. No caso de óxidos (cristais e vidros) geralmente a contribuição do efeito de LP é muito maior que o devido a LT. Entretanto, os materiais fluoretos (cristais e vidros) geralmente apresentam ?a e condutividade térmica muito menores que os óxidos, o que diminui o efeito de LP e aumenta a LT. Consequentemente, a LP e LT podem dar contribuições de mesma magnitude ao sinal de Z-scan. Nestes casos, é preciso discriminar estes dois efeitos para determinar ? a .

$\checkmark$ Capítulo 7 - Conclusão.

Gostaria de observar que, esta tese dá continuidade a uma linha de trabalhos que vem sendo desenvolvidos com o objetivo de caracterizar materiais por meio das técnicas de Lente Térmica e Zscan. Os trabalhos são desenvolvidos em nosso grupo, Espectroscopia de Sólidos em conjunto com o grupo de Fototérmica da Universidade Estadual de Maringá. As técnicas de Lente Térmica e Zscan têm sido aplicadas no estudo de diversos materiais, tais como: vidros (fluoretos, calcohaletos, calcogenetos, silicatos (puro e dopados com $\mathrm{Nd}^{3+}$ ) e aluminato de cálcio (dopado com $\mathrm{Nd}^{3+} \mathrm{e}$ $\left.\mathrm{Er}^{3+}\right)$; cristais dopados com $\mathrm{Nd}^{3+}\left(\mathrm{YAG}, \mathrm{YVO}_{4}\right.$ e YLF) e com $\mathrm{Cr}^{3+}(\mathrm{LiSAF}, \mathrm{LiSGaF}$ $\mathrm{Al}_{2} \mathrm{O}_{3}$ (Rubi) e $\mathrm{BeA}_{12} \mathrm{O}_{4}$ (Alexandrita)). Recentemente, a técnica de Lente Térmica 
também vem sendo aplicada na caracterização de cristais líquidos [5, 7-9, 12, 15-44] e polímeros [25-27, 41, 45]. Isto mostra a grande potencialidade destas duas técnicas na caracterização de propriedade em materiais utilizados como meio ativo e/ou amplificadores óticos.

\subsection{Características espectrocópicas de sólidos dopados com $\mathrm{Nd}^{3+}$.}

Os íons terras raras têm uma longa história em aplicações óticas, e dentre ele o que mais se destacou, principalmente como meio ativo para laser, foi o $\mathrm{Nd}^{3+}$. Embora não tenha sido o primeiro íon a emitir luz em um sólido, ele se tornou o mais importante íon ativador para laseres. O interesse por este íon vem do fato dele apresentar um sistema de quatro níveis quando inserido em matrizes cristalinas ou vítreas. Num sistema de três níveis pode ocorrer reabsorção do fóton emitido entre o nível metaestável e o nível fundamental, enquanto que num sistema de quatro níveis a probabilidade de ocorrer a eabsorção é pequena, pois a transição ocorre entre o níveis metaestáveis e um nível intermediário. Outra vantagem do uso do $\mathrm{Nd}^{3+} \mathrm{em}$ meio ativo para laser é a alta seção de choque de absorção da banda em torno de $800 \mathrm{~nm}$, permitindo um bombeio com laser de diodo.

Para uma melhor compreensão dos laseres de $\mathrm{Nd}^{3+}$, é necessário uma certa familiaridade com os detalhes da estrutura dos níveis de energia do $\mathrm{Nd}^{3+}$. Referindose a Figura 1.1, o estado fundamental é o nível ${ }^{4} \mathrm{I}_{9 / 2} ;$ o espectro de absorção visível ocorre do estado fundamental para vários níveis do estado excitado. $\mathrm{O}$ nível ${ }^{4} \mathrm{~F}_{3 / 2}$ é o nível laser. Ele possui um tempo de vida radiativo tipicamente da ordem de $200-1100 \mu s$. Do nível ${ }^{4} \mathrm{~F}_{3 / 2}$ os átomos decaem radiativamente através de quatro 
possíveis transições $\quad\left[{ }^{4} \mathrm{~F}_{3 / 2} \rightarrow{ }^{4} \mathrm{I}_{15 / 2}(1,80 \mu \mathrm{m}), \quad{ }^{4} \mathrm{I}_{13 / 2}(1,35 \mu \mathrm{m}), \quad{ }^{4} \mathrm{I}_{11 / 2}(1,06 \mu \mathrm{m})\right.$, $\left.{ }^{4} \mathrm{I}_{9 / 2}(0,88 \mu \mathrm{m})\right]$. Note que a transição $\left({ }^{4} \mathrm{~F}_{3 / 2} \rightarrow{ }^{4} \mathrm{I}_{9 / 2}\right)$ é uma emissão ressonante com o estado fundamental. É muito comum em materiais laseres, as radiações $1,80 \mu \mathrm{m}$ e $1,35 \mu \mathrm{m}$ não serem detectadas por causa do forte processo de relaxação cruzada neste comprimento de onda no $\mathrm{Nd}^{3+}$ [46].

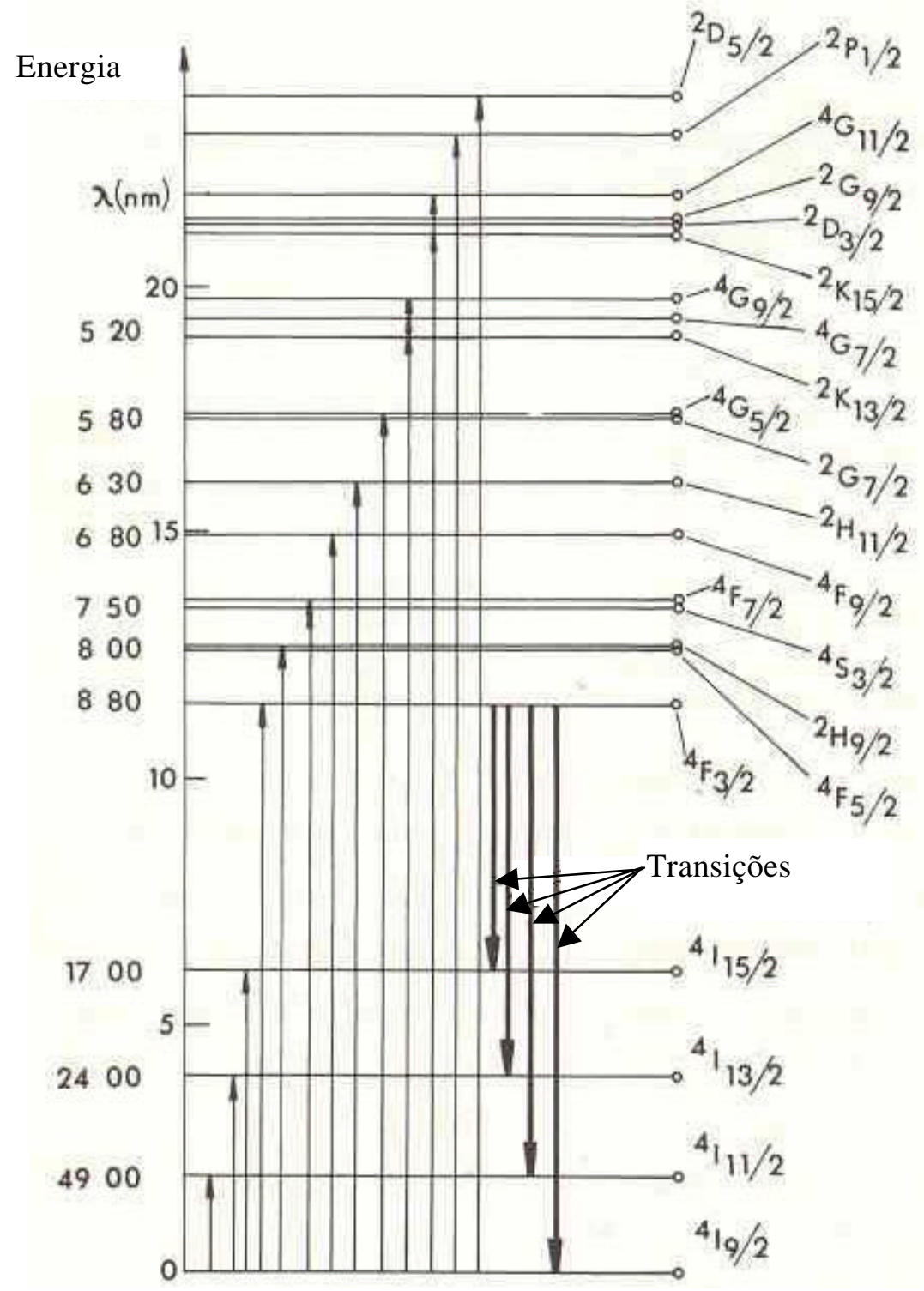

Figura 1.1 - Diagrama de níveis de energia do $\mathrm{Nd}^{3+}$. Figura tirada da referência [47].

Entre os laseres de estado sólido mais importantes temos o de $Y A G: N d^{3+}$, que é também o mais utilizado. Ele possui uma combinação de propriedades 
favoráveis para a operação laser, como por exemplo sistema de 4 níveis, boa qualidade ótica, alta condutividade térmica $(\sim 14 \mathrm{~W} / \mathrm{Kcm})$ e longo tempo de vida do nível emissor $(\sim 260 \mu \mathrm{s})$. Além disso, a sua estrutura cúbica favorece uma estreita largura de linha de fluorescência, a qual resulta em um alto ganho e um baixo limiar ("threshold") para a operação laser.

A Figura 1.2 ilustra os intervalos dos tempos de vida do nível laser emissor ${ }^{4} \mathrm{~F}_{3 / 2}$ para vários tipos de vidros encontrados na literatura [46]. Podemos observar que os silicatos exibem o maior intervalo, seguido pelos borosilicatos, enquanto que os menores intervalos ficam por conta dos vidros teluretos e fluorozirconatos.

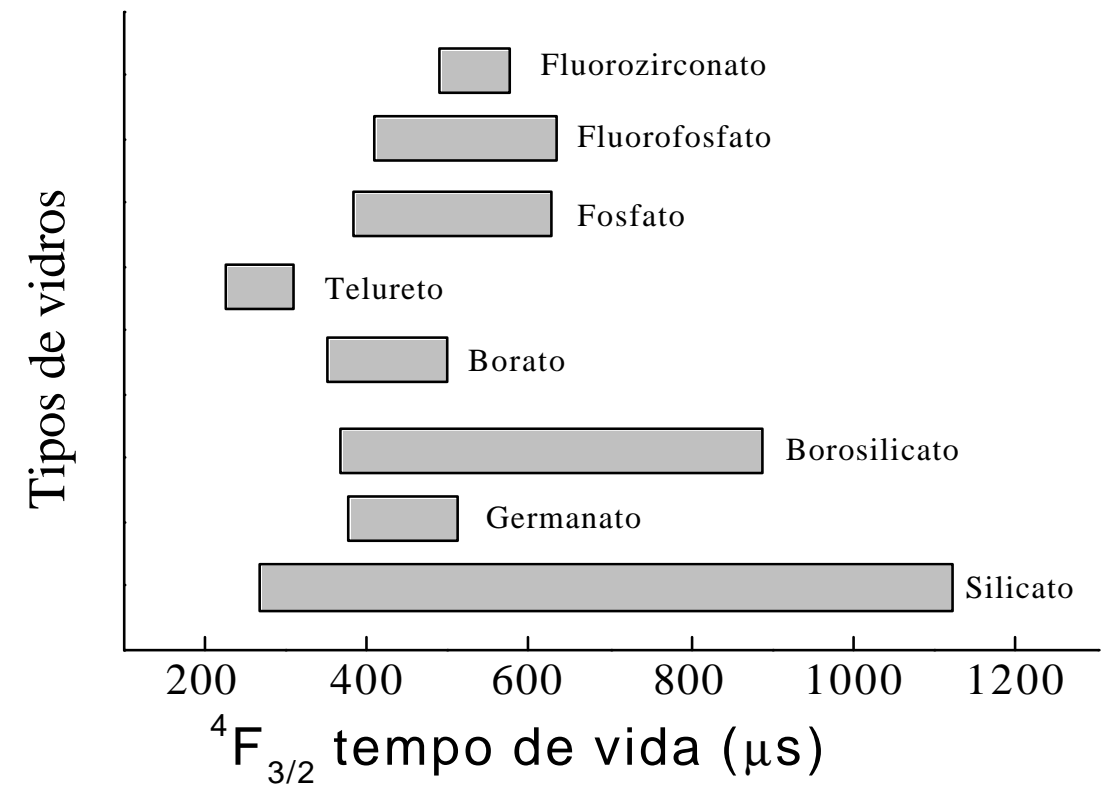

Figura 1.2 - Intervalo dos tempos de vida radiativo para o estado metaestável ${ }^{4} F_{3 / 2}$ [46].

A Figura 1.3 ilustra a emissão da transição ${ }^{4} \mathrm{~F}_{3 / 2} \rightarrow{ }^{4} \mathrm{I}_{11 / 2}$ para vários vidros dopados com $\mathrm{Nd}^{3+}$. Podemos observar que, de uma matriz para outra podem ocorrer deslocamentos, alargamentos ou estreitamentos da banda de emissão do íon. Isto é devido ao desdobramento e deslocamento dos níveis de energia do íon luminescente. 


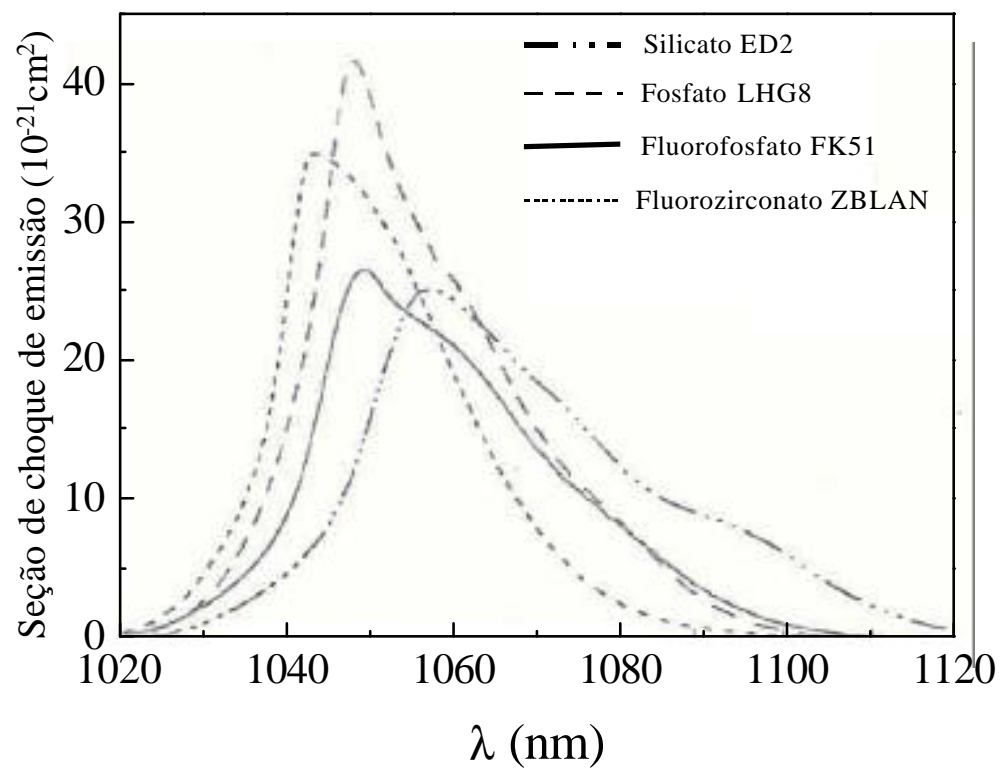

Figura 1.3 - Espectro da seção de choque de emissão da transição ${ }^{4} F_{3 / 2} \rightarrow{ }^{4} I_{11 / 2}$ para vários vidros [46].

Os materiais, vidros ou cristais, dopados com íons, podem apresentar mecanismos de perda que comprometem a sua operação laser. Um exemplo disto é a transferência de energia entre os íons, por "upconversion Auger" e relaxação cruzada, que resulta em um forte efeito de lente térmica no material [48-50]. Isto ocorre porque o processo de transferência de energia propicia os processos nãoradiativos, ao invés dos processos radiativos que são desejados para a obtenção do laser. Estes processos são discutidos no Capítulo 5.

\subsection{Investigação da origem da não-linearidade em sólidos dopados.}

Efeitos não-lineares são particularmente importantes em fibras óticas, onde a energia laser é concentrada no centro da fibra, produzindo uma alta intensidade de luz, mesmo no caso de uma onda contínua. Também os efeitos térmicos tal como a 
variação térmica do índice de refração, expansão térmica e a tensão termicamente induzida são importantes no projeto de laseres de estado sólido [51].

A técnica de Z-scan, utilizada neste trabalho, tem se mostrado muito eficiente no estudo de materiais onde a não-linearidade tem um tempo de resposta $>10^{-4} \mathrm{~s}[5$, 8-12, 52, 53]. Em sólidos dopados a não-linearidade origina-se da variação da população do estado metaestável do íon dopante, o qual apresenta uma susceptibilidade diferente do estado fundamental. A este processo denominamos de efeito de Lente de População (LP) [54]. Neste caso, a parte real do índice de refração não-linear é proporcional à diferença de polarizabilidade $\Delta \alpha$ entre o estado excitado e o estado fundamental, enquanto que, a parte imaginária do índice de refração nãolinear é proporcional à diferença de seção de choque de absorção ?s, entre o estado excitado e o estado fundamental [52]. Geralmente, parte da população do estado excitado decai via um processo não-radiativo, aquecendo a amostra e gerando uma variação no caminho ótico devido ao coeficiente de temperatura do índice de refração, dn/dT, ao coeficiente de expansão térmica ao longo do caminho ótico e aos efeitos fotoelásticos. Todos estes processos conduzem a uma mudança do caminho ótico com a temperatura, ds/dT, o qual causa o chamado efeito de Lente Térmica (LT) em sólidos (em líquidos ds/dT = dn/dT ) [6].

Entre os materiais dopados, o $\mathrm{Cr}^{3+}[4,10,11,53,55,56]$ e o $\mathrm{Nd}^{3+}[3,5]$ têm sido os mais estudados devido a suas aplicações em laseres [3, 12]. Estes íons foram estudados em diferentes matrizes hospedeiras. Foi observado que a parte real do índice de refração não-linear $\mathrm{n}_{2}{ }^{\prime}$ é tipicamente um ordem de magnitude maior do que a parte imaginária $\mathrm{n}_{2}{ }^{\prime \prime}$ e que ?a varia num intervalo de $10^{-26}-10^{-25} \mathrm{~cm}^{3}$, sendo maior nos óxidos do que nos fluoretos (cristais e vidros). 
Weaver e Powell $[4,56]$ usaram a técnica de mistura de quatro ondas para investigar a diferença de polarizabilidade ?a em diferentes cristais dopados com $\mathrm{Cr}^{3+}$. A Figura 1.4 mostra os resultados de ? a obtidos pelos autores acima em sólidos dopados com $\mathrm{Nd}^{3+}$. No caso dos cristais fluoretos, seus experimentos não apresentavam sensibilidade suficiente para determinar ?a, de forma que obtiveram apenas um limite superior para ? a . No caso dos sólidos dopados com $\mathrm{Nd}^{3+}$, ? a apresenta ser maior nos óxidos do que nos fluoretos. Devido aos fluoretos terem menor condutividade térmica do que os óxidos, eles têm um efeito de LT maior. Portanto, a grande dificuldade em medir ? a dos materiais fluoretos, não é somente por causa do pequeno valor de ? a mas também devido ao seu grande efeito de LT.

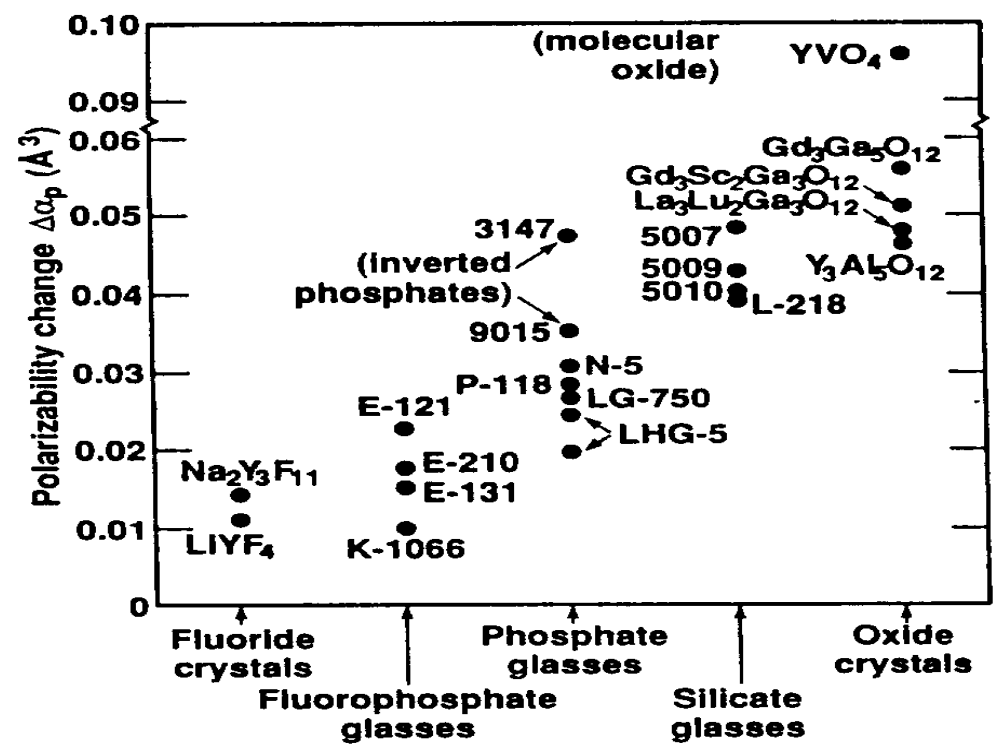

Figura 1.4 - ? a do $\mathrm{Nd}^{3+}$ em diversos materiais [4].

\subsection{Efeito não-linear eletrônico}

Quando um ion é bombeado em ressonância com uma linha de absorção, ambos efeitos LP e LT são proporcionais à população do estado excitado, $\mathrm{N}_{\mathrm{ex}}(\mathrm{t})$, cuja evolução temporal pode ser calculada através das equações de taxa. 
Para entendermos como se comportam os sólidos dopados, vamos considerar o sistema de diagrama de níveis generalizado da Figura 1.5 que pode ser usado para representar sólidos dopados $\mathrm{cm} \mathrm{Nd}^{3+}$ e $\mathrm{Cr}^{3+}$. Supomos que o sistema é excitado a partir do estado fundamental $g$ para um nível intermediário $\mathrm{I}_{1} \mathrm{o}$ qual decai rapidamente $(\sim \mathrm{ns})$ para um estado metaestável ex (com tempo de vida $\mathrm{t}_{0}$ da ordem de milisegundos). No caso do $\mathrm{Cr}^{3+}$ temos a banda ${ }^{4} \mathrm{~T}_{2}$ como nível intermediário $\left(\mathrm{I}_{1}\right)$ e o nível ${ }^{2} E$ (ex) decai diretamente para o estado fundamental $(g)$. Neste caso não existe o nível $\mathrm{I}_{2}$. No caso do $\mathrm{Nd}^{3+}$ temos $e x={ }^{4} \mathrm{~F}_{3 / 2}$, o qual pode decair diretamente para o estado fundamental $g$ ou para outro estado intermediário $\left(\mathrm{I}_{2}\right)$ que corresponde aos níveis ${ }^{4} \mathrm{I}_{15 / 2},{ }^{4} \mathrm{I}_{13 / 2}$ e ${ }^{4} \mathrm{I}_{1 / 2}$ (Figura 1.1). Entretanto, uma vez que a diferença de energia $? \mathrm{E}=\mathrm{I}_{2}-g$ é pequena $\left(\sim 2000 \mathrm{~cm}^{-1}\right)$, o sistema relaxa rapidamente para o estado fundamental com a emissão de fônons.

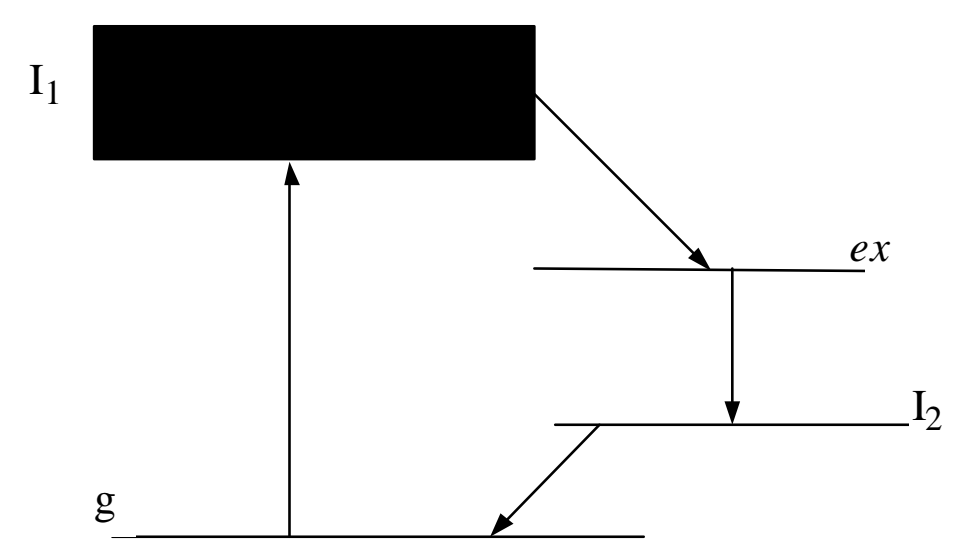

Figura 1.5 - Diagrama de um sistema genérico de 4 níveis.

Por meio das equações de taxa, podemos determinar as populações $\mathrm{N}_{\mathrm{g}}$ e $\mathrm{N}_{\mathrm{ex}}$ correspondentes aos níveis $g$ e ex. Uma vez que o tempo de vida dos níveis intermediários I é muito pequeno ( ns, muito menor que o tempo de vida do 
estado ex), podemos considerar $\mathrm{N}_{\mathrm{I}_{1}} \sim \mathrm{N}_{\mathrm{I}_{2}} \sim 0$. Portanto, trataremos apenas as populações dos estados excitado $\mathrm{N}_{\mathrm{ex}}$ e fundamental $\mathrm{N}_{\mathrm{g}}$. Além disso desprezaremos o decaimento direto do nível $\mathrm{I}_{1}$ para o nível fundamental $g$, pois a taxa de transição do nível $\mathrm{I}_{1}$ para o nível ex é muito maior. Desta forma podemos aproximar a 1 a eficiência quântica de bombeio do nível ex. Desta maneira temos:

$$
\begin{aligned}
& \frac{\mathrm{dN}_{\mathrm{g}}}{\mathrm{dt}}=-\frac{\mathrm{sI}}{\mathrm{h} ?} \mathrm{~N}_{\mathrm{g}}+\frac{\mathrm{N}_{\mathrm{ex}}}{\mathrm{t}_{0}} \\
& \frac{\mathrm{dN}_{\mathrm{ex}}}{\mathrm{dt}}=\frac{\mathrm{sI}}{\mathrm{h} ?} \mathrm{~N}_{\mathrm{g}}-\frac{\mathrm{N}_{\mathrm{ex}}}{\mathrm{t}_{0}} \\
& \mathrm{~N}_{0}=\mathrm{N}_{\mathrm{g}}+\mathrm{N}_{\mathrm{ex}}
\end{aligned}
$$

sendo I é a intensidade do laser de excitação, $t_{0}$ o tempo de vida espontâneo no estado metaestável $e x, h$ ? a energia do fóton de excitação e $\mathrm{s}$ a seção de choque desta transição. Como a população total $\mathrm{N}_{0}$ é constante devemos ter

$$
\frac{\mathrm{dN}_{\mathrm{g}}}{\mathrm{dt}}=-\frac{\mathrm{dN}_{\mathrm{ex}}}{\mathrm{dt}}
$$

como pode ser observado por meio das equações (1.1) e (1.3). Substituindo a equação (1.3) em (1.2) obtemos:

$$
\frac{\mathrm{dN}_{\mathrm{ex}}}{\mathrm{dt}}=\mathrm{S} \frac{\mathrm{N}_{0}}{\mathrm{t}_{0}}-\frac{(1+\mathrm{S})}{\mathrm{t}_{0}} \mathrm{~N}_{\mathrm{ex}}
$$

sendo $\mathrm{S}=\mathrm{I} / \mathrm{I}_{\mathrm{s}}$ é o parâmetro de saturação e $\mathrm{I}_{\mathrm{s}}$ é a intensidade de saturação dada por:

$$
\mathrm{I}_{\mathrm{s}}=\frac{\mathrm{hc}}{? \mathrm{st}_{0}}
$$

Supondo que em $\mathrm{t}=0$ o sistema está no estado fundamental, ou seja, $\mathrm{N}_{\mathrm{g}}=\mathrm{N}_{0}$ e $\mathrm{N}_{\mathrm{ex}}=0$, e neste instante é ligado o laser com intensidade $\mathrm{I}$, das equações (1.2) e (1.5) obtemos: 


$$
\begin{aligned}
& N_{e x}(t)=N_{0} \frac{S}{1+S}\left(1-e^{-t / t}\right) \\
& N_{g}(t)=N_{0} \frac{S}{1+S}\left(1-e^{-t / t}\right) \\
& t=\frac{t_{0}}{1+S}
\end{aligned}
$$

Devido a distribuição da população entre o estado excitado (ex) e o fundamental ( $g$ ) a susceptibilidade do meio fica dada por:

$$
?=\frac{\mathrm{N}_{\mathrm{g}} ?_{\mathrm{g}}+\mathrm{N}_{\mathrm{ex}} ?_{\mathrm{ex}}}{\mathrm{N}_{0}}+?_{\mathrm{m}}
$$

que pela Eq. (1.3) podemos rescrever como:

$$
?=?_{\mathrm{m}}+?_{\mathrm{g}}+\left(?_{\mathrm{ex}}-?_{\mathrm{g}}\right) \frac{\mathrm{N}_{\mathrm{ex}}}{\mathrm{N}_{0}}
$$

sendo $?_{\mathrm{j}}$ a susceptibilidade do íon no estado $j=g$ ou ex e $?_{\mathrm{m}}$ a contribuição da matriz hospedeira (cristal ou vidro). A polarizabilidade do estado $j$ é dada pela parte real de $?_{\mathrm{j}}$ normalizada pela concentração $\mathrm{N}_{0}$ :

$$
\mathrm{a}_{\mathrm{j}}=\operatorname{Re}\left\{?_{\mathrm{j}}\right\} / \mathrm{N}_{0}
$$

A parte imaginária de $?_{\mathrm{j}}$ é proporcional a seção de choque de absorção $\mathrm{s}_{\mathrm{j}}$, o qual está relacionado com o coeficiente de absorção da intensidade da luz (lei de Beer) $A_{j}=N_{0} s_{j}$, ou seja

$$
\mathrm{s}_{\mathrm{j}}=f_{\mathrm{L}}^{2} \operatorname{Im}\left\{?_{\mathrm{j}}\right\} / \mathrm{N}_{0}
$$

com

$$
f_{\mathrm{L}}=\frac{\mathrm{n}_{0}^{2}+2}{3}
$$

sendo $f_{L}$ o fator de Lorenz, que é responsável pela correção devido ao efeito do campo local no dielétrico. 
O índice de refração complexo $n$ pode ser calculado, a partir de ?, através da equação de Clausius-Mossotti (no sistema CGS):

$$
\frac{\mathrm{n}^{2}-1}{\mathrm{n}^{2}+2}=\frac{4 \mathrm{p}}{3}\left(\frac{\mathrm{N}_{\mathrm{g}} ?_{\mathrm{g}}+\mathrm{N}_{\mathrm{ex}} ?_{\mathrm{ex}}}{\mathrm{N}_{0}}+?_{\mathrm{m}}\right)
$$

Supondo que a variação de índice de refração é pequena, podemos escrever $\mathrm{n}=\mathrm{n}_{0}+? \mathrm{n}$ com $? \mathrm{n}<<\mathrm{n}_{0}$, sendo $\mathrm{n}_{0}$ o índice de refração na ausência do efeito não-linear, ou seja, quando $\mathrm{I}=0 \quad\left(\mathrm{~N}_{\mathrm{g}}=\mathrm{N}_{0}\right.$ e $\left.\mathrm{N}_{\mathrm{ex}}=0\right)$. Como supomos que ? $\mathrm{n}<<\mathrm{n}_{0}$, podemos expandir (1.15) e usando (1.7) e (1.8) temos:

$$
\begin{aligned}
& \frac{\mathrm{n}_{0}^{2}-1}{\mathrm{n}_{0}^{2}+2}=\frac{4 \mathrm{p}}{3}\left(?_{\mathrm{g}}+?_{\mathrm{m}}\right) \\
& ? \mathrm{n}=\frac{2 \mathrm{p}}{9} f_{\mathrm{L}}^{2}\left(?_{\mathrm{ex}}-?_{\mathrm{g}}\right)\left(\frac{\mathrm{S}}{1+\mathrm{S}}\right)\left(1-\mathrm{e}^{-\mathrm{t} / \mathrm{t}}\right)
\end{aligned}
$$

No caso estacionário $(\mathrm{t}>\mathrm{t}$ ) podemos escrever:

$$
\mathrm{n}=\mathrm{n}_{0}+\frac{\mathrm{n}_{2} \mathrm{I}}{1+\mathrm{S}}
$$

com

$$
\mathrm{n}_{2}=\frac{2 \mathrm{p}}{\mathrm{n}_{0}} f_{\mathrm{L}}^{2}\left(\frac{?_{\mathrm{ex}}-?_{\mathrm{g}}}{\mathrm{I}_{\mathrm{s}}}\right)
$$

A parte real de $\mathrm{n}_{2}=\mathrm{n}_{2}{ }^{\prime}-$ in ${ }_{2}$ " é proporcional a diferença de polarizabilidade $? \mathrm{a}=\mathrm{a}_{\mathrm{ex}}-\mathrm{a}_{\mathrm{g}}$ entre os estados ex e $g$. Utilizando as Eqs. (1.19) e (1.12) obtemos:

$$
\mathrm{n}_{2}^{\prime}=\frac{2 \mathrm{p} f_{\mathrm{L}}^{2}}{\mathrm{n}_{0}} \mathrm{~N}_{0} \frac{? \mathrm{a}}{\mathrm{I}_{\mathrm{s}}}
$$

A parte imaginária de $\mathrm{n}_{2}$ pode ser escrita em termo das seções de choque $\mathrm{s}_{\mathrm{ex}}$ e $\mathrm{s}_{\mathrm{g}}$ dos estados excitados e fundamental como: 


$$
\mathrm{n}_{2}^{\prime \prime}=\frac{?}{4 \mathrm{p}} \mathrm{N}_{0} \frac{? \mathrm{~s}}{\mathrm{I}_{\mathrm{s}}}
$$

com ? $\mathrm{s}=\mathrm{s}_{\mathrm{ex}}-\mathrm{s}_{\mathrm{g}}$ sendo a diferença entre as seções de choque de absorção dos dois estados e ? o comprimento de onda. Portanto, a redistribuição das populações $\mathrm{N}_{\mathrm{g}}$ e $\mathrm{N}_{\mathrm{ex}}$ provoca uma alteração na parte real do índice de refração do meio.

As expressões acima são muito importantes neste trabalho, pois elas permitem a determinação de quantidades microscópicas como ?a e ?s, através de medidas do parâmetro $\mathrm{n}_{2}$. Deve-se notar ainda a grande importância do fator de Lorenz $f_{L}$, principalmente nos cristais óxidos que apresentam um alto índice de refração $\left(\mathrm{n}_{0} \sim 2\right)$ e consequentemente um alto valor de $f_{L}$. 


\section{Capítulo 2}

\section{Espectrometria de LT}

As propriedades espectroscópicas, como por exemplo, eficiência quântica e tempo de vida da fluorescência desempenham papel fundamental na escolha de um material para laser de estado sólido. As propriedades térmicas, tais como difusividade térmica e condutividade térmica, também são importantes nessa escolha, já que durante a operação laser ocorre uma variação de temperatura no meio ativo, que pode distorcer o feixe do laser e consequentemente reduzir o seu desempenho.

Este capítulo será dedicado à apresentação da técnica de Lente Térmica (LT), isto é, a teoria envolvida e a montagem experimental utilizada. Também serão apresentados resultados obtidos para as propriedade térmicas: difusividade térmica e condutividade térmica.

\subsection{Técnica de Lente Térmica (LT)}

O efeito de lente é causado pela deposição de calor por um processo de decaimento não-radiativo, após a luz do laser ter sido absorvida pela amostra, gerando uma distribuição de temperatura. Quando um laser contínuo incide na amostra, ela vai se esquentando e ao mesmo tempo o calor vai se difundindo radialmente (no caso de excitação por um feixe gaussiano TEM $_{00}$ que tem simetria radial) tal como mostra a Figura 2.1. Este processo tem um tempo característico dado por: 


$$
\mathrm{t}_{\mathrm{c}}=\frac{\mathrm{w}_{\mathrm{e}}^{2}}{4 \mathrm{D}}
$$

sendo $\mathrm{w}_{\mathrm{e}}(\mathrm{cm})$ a cintura do feixe de excitação na amostra, $\mathrm{D}=\mathrm{k} /$ ? $\mathrm{c}$ a difusividade térmica, ? $\left(\mathrm{gcm}^{-3}\right)$ a densidade, $\mathrm{c}\left(\mathrm{Jg}^{-1} \mathrm{~K}^{-1}\right)$ o calor específico e $\mathrm{k}(\mathrm{W} / \mathrm{Kcm})$ a condutividade térmica. Podemos observar pela Figura 2.1 que o aumento da temperatura é maior no centro do feixe e tende a zero para valores de $\mathrm{r} / \mathrm{w}_{\mathrm{e}}$ muito maiores do que a unidade. Além disso, a temperatura aumenta inicialmente com o tempo, tendendo ao estado estacionário quando $\mathrm{t}>>\mathrm{t}_{\mathrm{c}}$. Podemos estimar a variação de temperatura ? T sofrida no centro da amostra após a radiação laser ser absorvida. Como exemplo tomemos um vidro fluoreto com $\mathrm{k} \sim 7 \times 10^{-3} \mathrm{~W} / \mathrm{Kcm}$, coeficiente de absorção $A=1 \mathrm{~cm}^{-1}$ e sendo excitado por uma potência de $\mathrm{P}=100 \mathrm{~m} \mathrm{~W}$, para um $\mathrm{t} / \mathrm{t}_{\mathrm{c}}=25$ temos que $? \mathrm{~T} \sim 1,1{ }^{0} \mathrm{C}$.

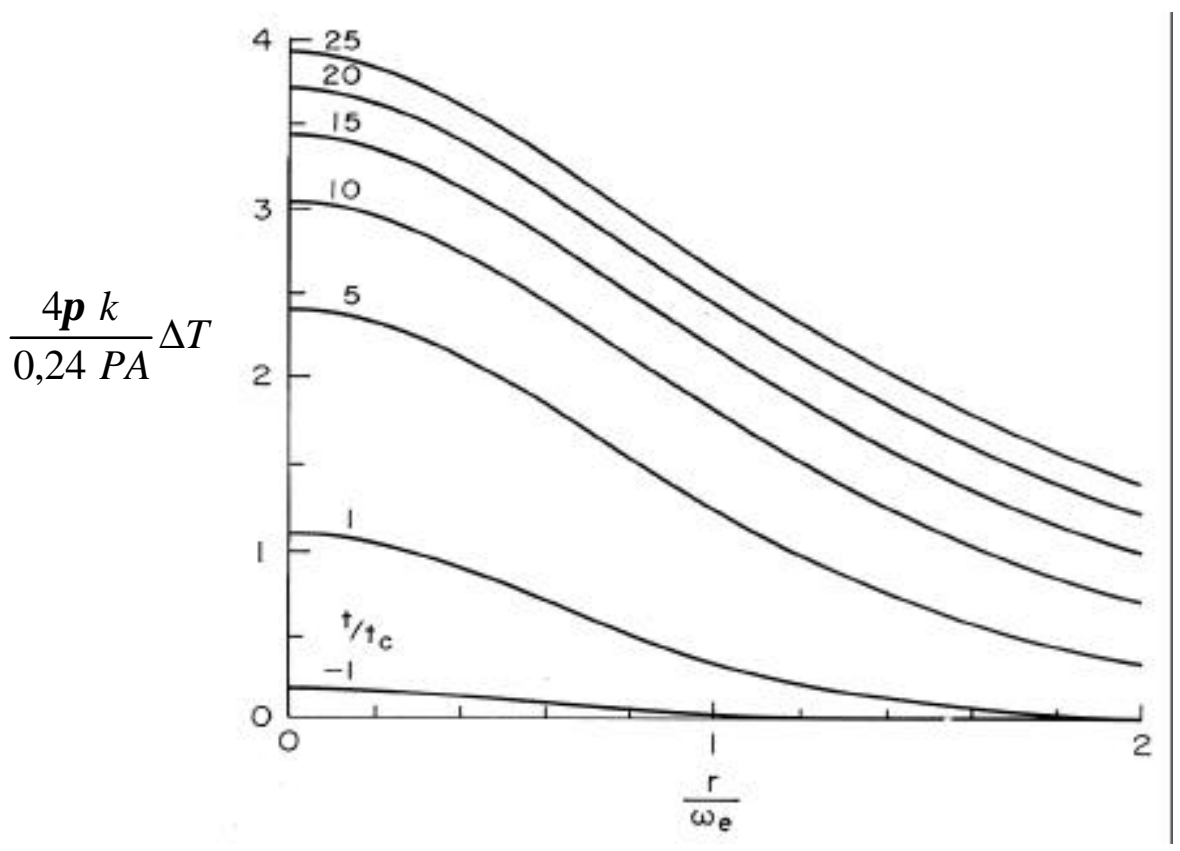

Figura 2.1 - Distribuição de temperatura ?T(r) na LT em função do tempo [57]. Sendo $P(m W)$ a potência do laser de excitação, $A\left(\mathrm{~cm}^{-1}\right)$ o coeficiente de absorção da amostra. 
No primeiro tratamento teórico do efeito de LT foi usado a chamada aproximação parabólica, onde a evolução temporal do perfil de temperatura ?T(r) e consequentemente, o perfil do índice de refração ?n(r) são dados por uma parábola. Neste caso, ?n(r) é equivalente a uma lente fina com distância focal $f$ a qual no regime estacionário é dada por [58]

$$
f^{-1}=2,3 \frac{\mathrm{PAL} \mathrm{dn} / \mathrm{dT}}{\mathrm{pkn}_{0} \mathrm{w}^{2}}
$$

com L (cm) sendo a espessura da amostra, $\mathrm{n}_{0} \mathrm{o}$ índice de refração inicial e $\mathrm{dn} / \mathrm{dT}\left(\mathrm{K}^{-1}\right)$ o coeficiente do índice de refração com a temperatura, $\mathrm{P}(\mathrm{mW})$ a potência do laser de excitação e $\mathrm{A}\left(\mathrm{cm}^{-1}\right)$ o coeficiente de absorção da amostra. Em líquidos, $\mathrm{dn} / \mathrm{dT}$ é geralmente negativo, tal que a LT é equivalente a uma lente divergente. A mudança da divergência de um laser passando através de uma amostra, devido ao efeito de LT, pode ser determinada através da medida da intensidade no centro do feixe no campo distante. Isto é feito colocando um "pinhole" na frente do detetor que se encontra no campo distante. A mudança relativa no sinal do detetor é proporcional a $f^{-1}$ ou a $\mathrm{k}^{-1} \mathrm{~A}(\mathrm{dn} / \mathrm{dT})$ como mostra a Eq. (2.2).

Embora a aproximação parabólica descreva qualitativamente as principais características da LT, ela não é quantitativamente correta, ou seja, fornece resultados de difusividade e $\mathrm{dn} / \mathrm{dT}$ errados (fator de $\approx 50 \%$ ), como mostrado mais tarde por Sheldon e colaboradores [57]. Eles usaram a integral de difração de Kirchhoff para calcular o perfil do feixe no campo distante, o qual foi chamado modelo aberrante, pois considera termo de ordem superior a 2 em $(r / w)^{\mathrm{n}}$, sendo equivalente as aberrações óticas. 
Os experimentos de LT podem ser feitos tanto na configuração de feixe único quanto de feixe duplo (excitação e prova), sendo que no segundo caso pode ser na configuração casada ou descasada [6]. Na configuração de feixe duplo, um feixe de maior intensidade é utilizado para provocar a LT e outro de menor intensidade para provar a LT criada. Na configuração descasada, o feixe de maior intensidade (feixe de excitação) tem sua cintura na amostra e o de menor intensidade (feixe de prova) tem a amostra na sua posição confocal (Figura 2.2). A cintura do feixe de prova é $\mathrm{w}_{\mathrm{op}}$, e os raios do feixe de prova e de excitação na amostra são $\mathrm{w}_{\mathrm{p}}$ e $\mathrm{w}_{\mathrm{e}}$, respectivamente. No caso da configuração de feixe simples, o feixe de excitação faz simultaneamente o papel de excitação e de prova, tal como no experimento de Zssan convencional. Neste caso tem-se $\mathrm{w}_{\mathrm{e}}=\mathrm{w}_{\mathrm{p}}$. A vantagem, no caso da configuração de modo descasado, em relação ao modo casado ou de feixe simples, é que a sensibilidade pode ser significativamente aumentada $\operatorname{com} \mathrm{w}_{\mathrm{p}}>\mathrm{w}_{\mathrm{e}}$. Este aumento na sensibilidade é uma conseqüência da difusão de calor que faz com que o perfil do índice de refração ?n(r) seja mais longo do que o perfil de intensidade do feixe de excitação. A partir da utilização de um pequeno orifício posicionado na frente do detetor do feixe de prova, a intensidade do centro do feixe $\left(r_{1}=0\right)$ pode ser monitorada e associada à absorção do material, permitindo a determinação quantitativa de suas propriedades termo óticas tais como: difusividade térmica, $D$, variação do caminho ótico com a temperatura, ds/dT e $\varphi$ a fração de energia convertida em calor. 


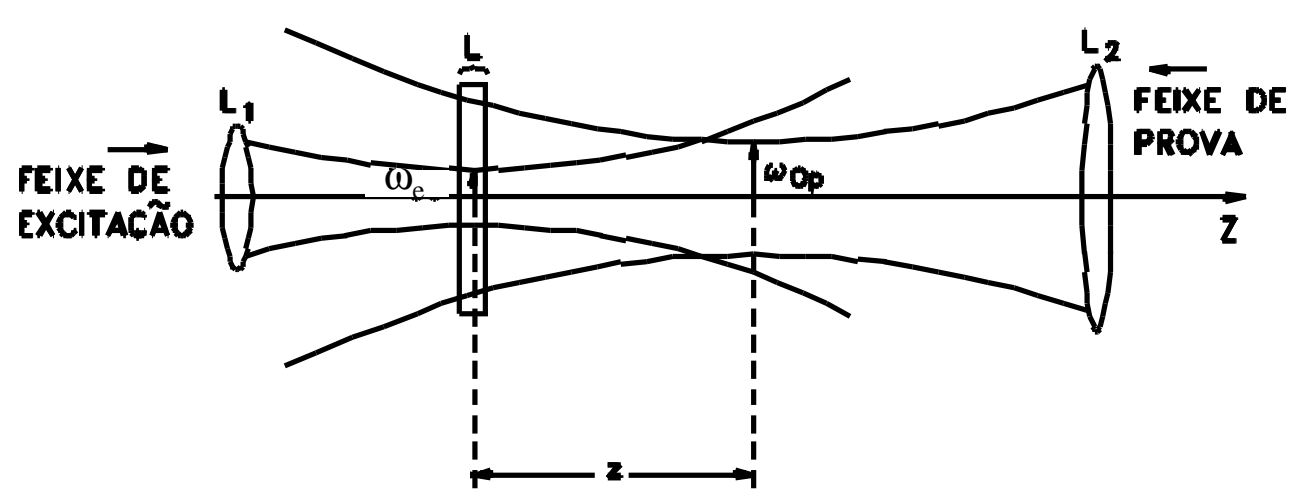

Figura 2.2 - Arranjo dos feixes de excitação e prova na configuração do experimento de LT com feixe duplo de modo descasado, onde $L_{1}$ e $L_{2}$ são as lentes e $\mathrm{w}_{\mathrm{e}}$ e $\mathrm{w}_{\mathrm{op}}$ os raios dos feixes de excitação e prova respectivamente.

O modelo aberrante foi generalizado para o caso de modo descasado por Shen e colaboradores [59] e a seguinte expressão para a intensidade no detetor foi obtida:

$$
\mathrm{I}(\mathrm{t})=\mathrm{I}(0)\left[1+\frac{?}{2} \tan ^{-1}\left(\frac{2 \mathrm{mV}}{\left[(1+2 \mathrm{~m})^{2}+\mathrm{V}^{2}\right](\mathrm{t} / 2 \mathrm{t})+1+2 \mathrm{~m}+\mathrm{V}^{2}}\right)\right]^{2}
$$

onde

$$
\mathrm{m}=\left(\frac{\mathrm{w}_{\mathrm{p}}}{\mathrm{w}_{\mathrm{e}}}\right)^{2}, \quad \mathrm{~V}=\frac{\mathrm{z}}{\mathrm{z}_{\mathrm{cp}}}, \quad \mathrm{t}_{\mathrm{c}}=\frac{\mathrm{w}_{\mathrm{e}}^{2}}{4 \mathrm{D}}
$$

$\mathrm{z}(\mathrm{cm})$ é a distância entre a cintura do laser de prova e a amostra, $\mathrm{z}_{\mathrm{cp}}(\mathrm{cm}) \quad \mathrm{o}$ parâmetro confocal do feixe de prova e $\mathrm{I}(0)$ o valor de $\mathrm{I}(\mathrm{t})$ quando $t$ ou $\theta$ é zero. A amplitude do sinal de LT, $\theta$, é proporcional a sua variação de fase dada por [6, 13]:

$$
?=-\frac{\mathrm{PAL}_{\mathrm{eff}}}{\mathrm{k} ?_{\mathrm{p}}} \varphi \frac{\mathrm{ds}}{\mathrm{dT}}
$$

sendo $\mathrm{P}(\mathrm{mW})$ a potência do feixe de excitação, $\mathrm{A}\left(\mathrm{cm}^{-1}\right)$ o coeficiente de absorção da amostra, $\lambda_{\mathrm{p}}(\mathrm{nm})$ o comprimento de onda do feixe de prova, $\mathrm{L}_{\text {eff }}=\left(1-\mathrm{e}^{-\mathrm{AL}}\right) / \mathrm{A}$ a espessura efetiva e L (cm) a espessura da amostra. A Figura 2.3 mostra um sinal 
típico de LT para a o vidro aluminato de cálcio (LSCAS ). Os dados são ajustados pela Eq. (2.3), de onde se obtém o parâmetro $?=-(0,2018 \pm 0,0001) \mathrm{rad}$ e $t_{c}=(0,685 \pm 0,001) \mathrm{ms}$. Usando este valor de $t_{c}$ juntamente com o valor medido de $\mathrm{w}_{\mathrm{e}}=(3,80 \pm 0,04) \times 10^{-3} \mathrm{~cm}$ na expressão $\mathrm{D}=\mathrm{w}_{\mathrm{e}}^{2} / 4 \mathrm{t}_{\mathrm{c}} \quad$ (Eq. (2.4)), nós obtemos $\mathrm{D}=(5,3 \pm 0,1) \times 10^{-3} \mathrm{~cm}^{2} / s$

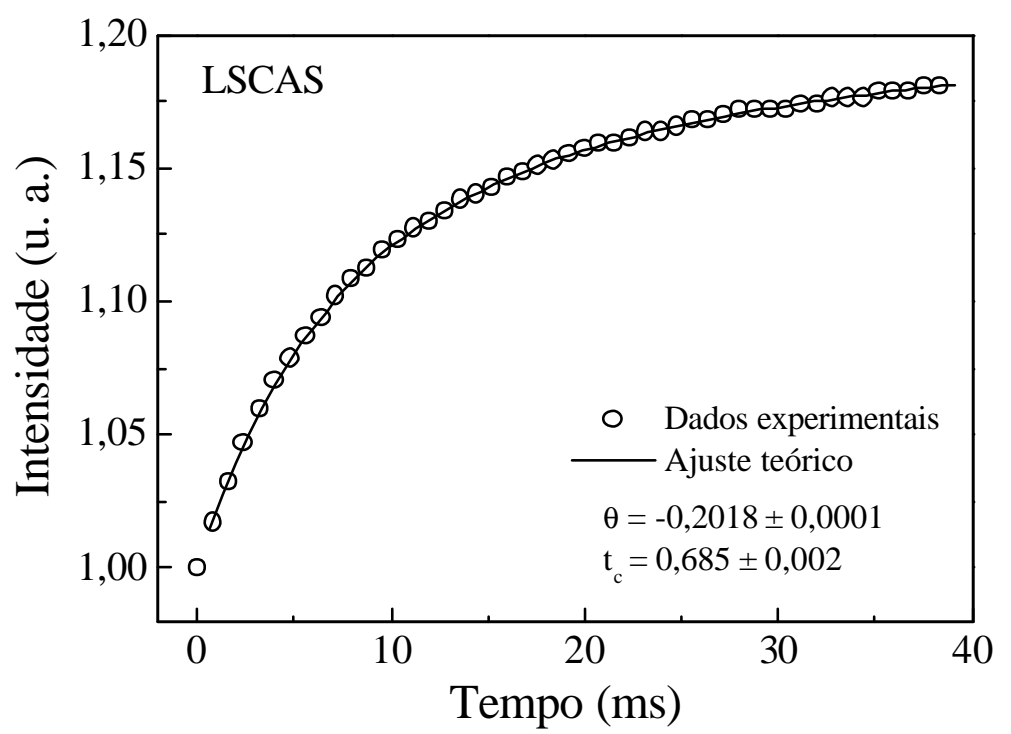

Figura 2.3 - Curva da evolução temporal do efeito de LT em uma amostra de LSCAS dopada com $2 \% \mathrm{Nd}^{3+}$, com ds $/ d T$ positivo. Com $A=3,3 \mathrm{~cm}^{-1}$ e $L=0,38 \mathrm{~cm}$. A medida foi realizada com $?_{\text {exc }}=810 \mathrm{~nm}$ e $P=69 \mathrm{~mW}$. Ajuste teórico realizado com a Eq. (2.3), com $m=23$ e $V=1,73$.

Na prática observa-se que os parâmetros $\theta$ e $t_{c}$ dependem ligeiramente do intervalo de tempo utilizado no ajuste. Por exemplo, para $t_{f}=40 \mathrm{~ms}$ obtém-se $\mathrm{t}_{\mathrm{c}}=0,685 \mathrm{~ms}, \quad ?=-0,2018 \mathrm{rad}, \quad$ enquanto que para $t_{f}=20 \mathrm{~ms}$ obtém-se $\mathrm{t}_{\mathrm{c}}=0,66 \mathrm{~ms}$ e $?=-0,2 \mathrm{rad}$. Observa-se que os valores de $\mathrm{t}_{\mathrm{c}}$ e $\theta$ tendem a estabilizar para $t_{f}$ longo (Figura 2.4).

A Figura 2.5 mostra que o erro relativo do ajuste diminui significativamente para $t_{f}>30 t_{c}$. Por isso, fizemos a maioria dos ajuste com $t_{f} \sim 40 t_{c}$. Outro fator que 
pode influenciar nos resultados da difusividade térmica é a não homogeneidade da amostra. Em amostras com qualidade ótica não muito boa, há uma variação de até $\sim 20 \%$ no valor da difusividade térmica.

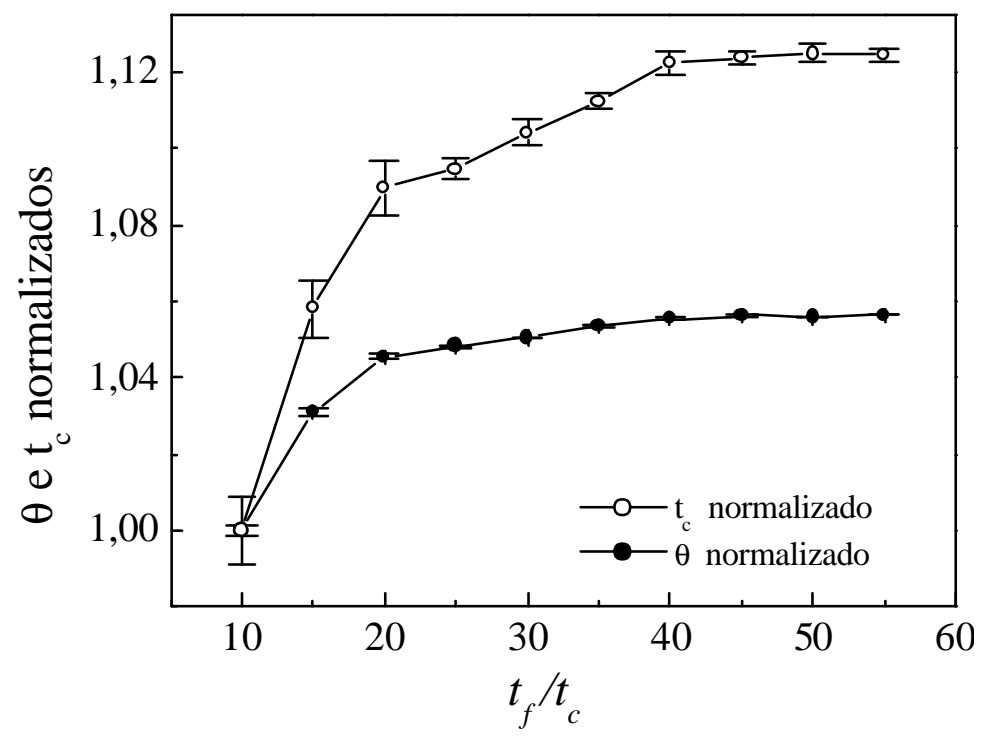

Figura 2.4 - $\theta$ e $t_{c}$ normalizados versus $t / t_{c}$.

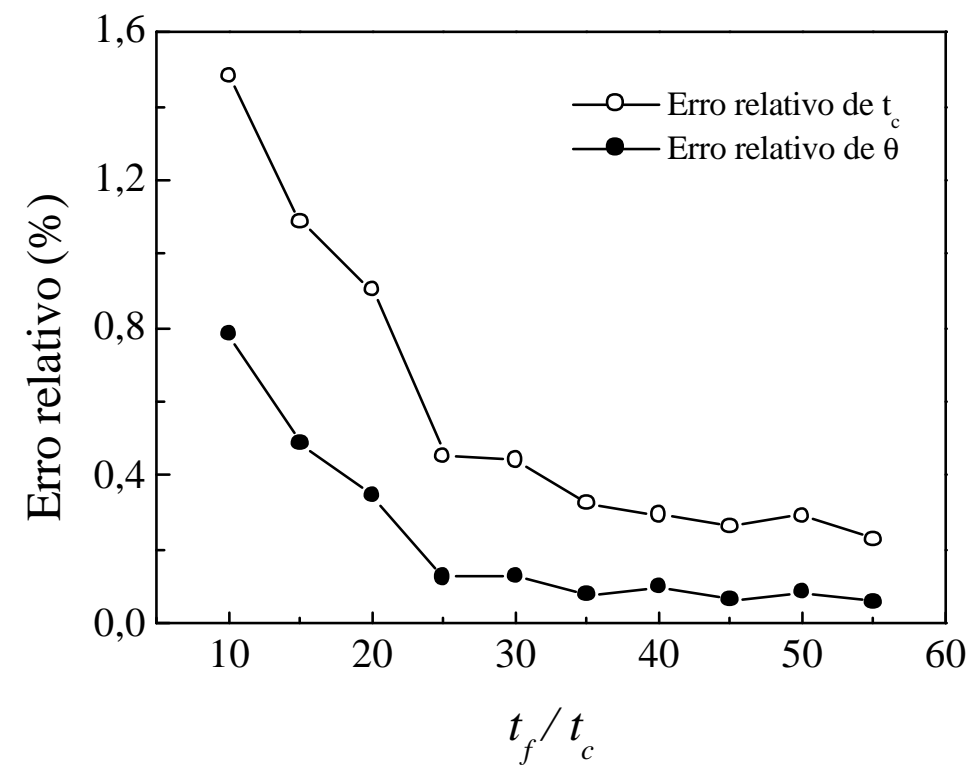

Figura 2.5 - Erro relativo de $\theta$ e $t_{c}$ versus $t / t_{c}$

$\mathrm{Na}$ grande maioria dos líquidos, o índice de refração diminui com a temperatura de modo que teremos dn/dT (variação do índice de refração com a temperatura) negativo e a lente formada provocará uma divergência do feixe laser. 
Em sólidos, dependendo da composição da amostra, dn/dT pode ser negativo ou positivo. No caso de amostras que apresentem alta mudança de polarizabilidade com a temperatura, dn/dT tende a ser positivo e assim o feixe laser é convergido após passar pela amostra.

É importante observar que com o parâmetro $\theta$ obtido do ajuste dos dados experimentais, nós obtemos $\varphi \mathrm{ds} / \mathrm{dT}$. Por exemplo no caso do LSCAS (Figura 2.3), $\varphi \mathrm{ds} / \mathrm{dT}=4,3 \times 10^{-6} \mathrm{~K}^{-1}$, onde $\mathrm{k}=1,53 \times 10^{-2} \mathrm{~W} / \mathrm{Kcm}$.

O termo $\varphi$ representa a fração de energia que é transformada em calor, sendo dada por:

$$
\varphi=1-? \frac{\left\langle ?_{\mathrm{em}}\right\rangle}{?_{\mathrm{exc}}}=1-?\left\langle ?_{\mathrm{em}}^{-1}\right\rangle ?_{\mathrm{exc}}
$$

em que $?_{\text {exc }}(\mathrm{Hz})$ a freqüência de excitação, $\left\langle ?_{\text {em }}\right\rangle$ é a frequiência média de emissão, $?_{\text {exc }}(\mathrm{nm})$ o comprimento de onda de excitação, ? a eficiência quântica da fluorescência e $\left\langle ?_{\mathrm{em}}^{-1}\right\rangle$ o valor médio do inverso do comprimento de onda da emissão, $?_{\text {em }}$. Normalmente a diferença de energia entre a excitação e a emissão é transferida para o sistema via emissão de fônons gerando calor (vide Figura 2.6). Na literatura o termo $\mathrm{h}\left(?_{\mathrm{exc}}-?_{\mathrm{em}}\right)$ é conhecido como defeito quântico, parâmetro que deve ser minimizado em laseres, uma vez que ele gera calor. Além disso, o calor gerado aumenta se a eficiência quântica do estado excitado for diferente da unidade, isto é, $?<1$. Sendo $?=1,\left\langle ?_{\mathrm{em}}^{-1}\right\rangle=0,95 \mu \mathrm{m}^{-1}$ (valor típico em sólidos dopados com $\mathrm{Nd}^{3+}$ ) e $?_{\text {exc }}=810 \mathrm{~nm}$ temos $\varphi=0,23$, enquanto que para $?_{\text {exc }}=514 \mathrm{~nm}$ temos $\varphi=0,51$, um aumento de aproximadamente 2 vezes no calor gerado dentro do material. 


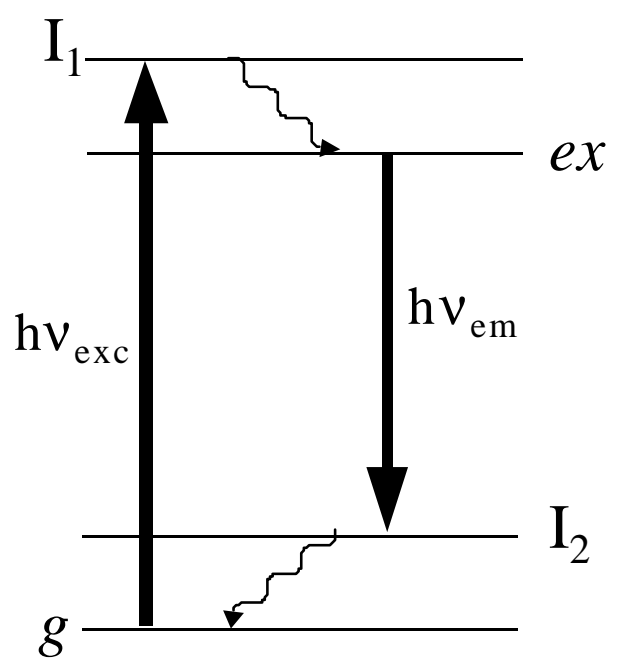

Figura 2.6 - Defeito quântico. Onde $g$ é o estado fundamental, $I_{1}$ e $I_{2}$ estados intermediários e ex o estado excitado.

\subsection{Montagem experimental.}

Na Figura 2.7 está esquematizado a montagem experimental para a LT na configuração no modo descasado. Em nossos experimentos utilizamos como laser de prova, um laser de HeNe marca Coherent, operando em 632nm com potência de $5 \mathrm{~mW}$ e como laser de bombeio um laser de $\mathrm{Ar}^{+}$marca Coherent, ou laseres sintonizáveis de corante Rhodamina $(?=540-630 \mathrm{~nm})$ e Ti-safira $(?=700-1100 \mathrm{~nm})$. Os laseres sintonizáveis eram excitados pelo lazer de $\mathrm{Ar}^{+} . \mathrm{A}$ montagem se dá da seguinte forma: o feixe laser de excitação é conduzido até a amostra pelos espelho M-1 e M-2, sendo focalizado por uma lente convergente de foco $f$. A amostra é posicionada na cintura do feixe de excitação, onde a intensidade é máxima, e ligeiramente deslocada da cintura do feixe de prova. O tempo de exposição da amostra ao feixe de excitação é controlada por um chopper, ch, cuja freqüência é variada de acordo com o tempo de resposta da amostra 
(dependendo de $\mathrm{w}_{\mathrm{e}}$ ). O feixe de excitação, após passar pela amostra, é atenuado por um filtro de densidade neutra, F-2, e incide no detetor D-1. Este sinal é utilizado para disparar o osciloscópio digital. O laser de $\mathrm{HeNe}$ é focado por uma lente convergente de foco maior do que a da lente do feixe de excitação, de forma que a suas posições focais fiquem descasadas [60]. O alinhamento é feito de maneira que o laser de HeNe cruze na cintura do feixe do laser de excitação, posição em que se encontra a amostra, com um ângulo de aproximadamente 1,5 graus. Ao passar pela amostra o feixe de $\mathrm{HeNe}$ percorre um longo caminho garantindo que apenas o centro do feixe do laser seja detectado pelo detetor D2. O sinal é armazenado por um osciloscópio digital Tektronix modelo TDS 210, que está conectado a um computador, onde é feito a aquisição e a análise dos dados. A utilização de filtros na frente dos detetores é necessário para eliminar sinais indesejáveis do laser de excitação no fotodiodo.

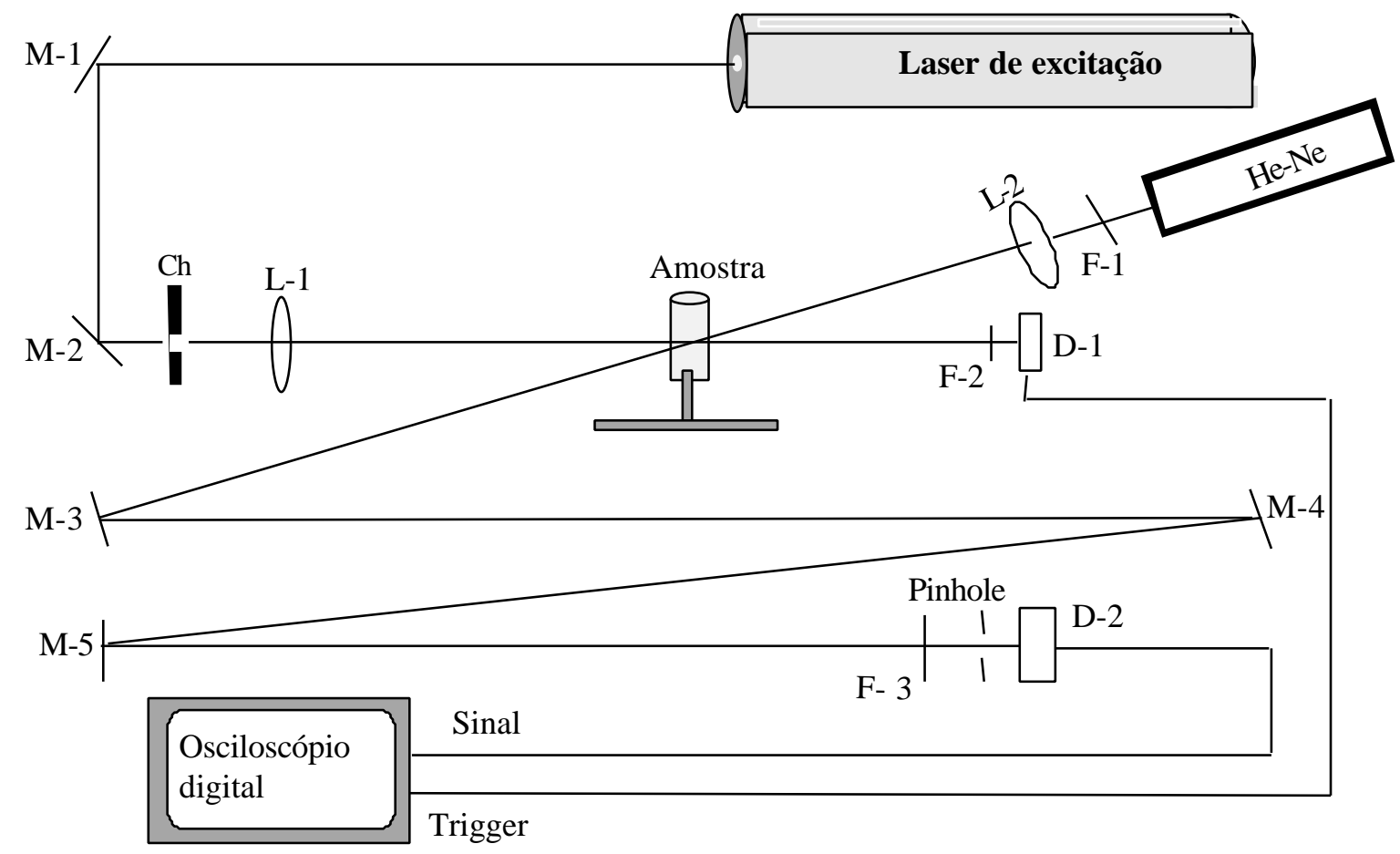

Figura 2.7 - Aparato experimental do experimento de LT. Onde M são os espelhos, L as lentes com distância focal $f \approx 20 \mathrm{~cm}, F$ os filtros, $D$ os detetores e ch o "chopper". 


\subsection{Determinação da cintura do feixe do laser.}

Para obter a cintura do feixe do laser e seus parâmetros confocais, utilizamos um medidor de diâmetro de feixe (Omega Meter - Beam Profiler, marca Thorblass, modelo WM100).

Da teoria sabemos que um feixe gaussiano $\mathrm{TEM}_{00}$, tem um perfil de intensidade dado por:

$$
\mathrm{I}(\mathrm{r})=\mathrm{I}_{0} \mathrm{e}^{-2 \mathrm{r}^{2} / \mathrm{w}^{2}}
$$

com

$$
\mathrm{w}^{2}=\mathrm{w}_{0}^{2}\left(1+\mathrm{z}^{2} / \mathrm{z}_{\mathrm{c}}^{2}\right)
$$

sendo $\mathrm{z}_{\mathrm{c}}=\mathrm{pw}_{0}^{2} /$ ? o chamado parâmetro confocal do feixe ${ }^{1}, \mathrm{w}_{0}$ a cintura do feixe no ponto de máxima ntensidade axial. Para obter-mos $\mathrm{w}_{0}$, nós medimos o diâmetro do feixe em várias posições na frente da lente, tanto da lente que focaliza o feixe de prova quanto na lente que focaliza o feixe de excitação (vide Figura 2.7), passando pelo seu foco e indo um pouco alem deste. Fazendo o gráfico do quadrado dos valores de $w$ obtido como o medidor, em função da distância, temos uma parábola, que pode ser ajustada pela equação $\mathrm{y}=\mathrm{a}+\mathrm{bz}+\mathrm{cz}^{2}$, fornecendo as constantes $a, b$ e $c$. O centro da parábola $\mathrm{z}_{1}=-\mathrm{b} / 2 \mathrm{c}$ indica a posição de menor diâmetro do feixe, normalmente $\mathrm{z}_{1} \approx f$ onde $f$ é a distância focal da lente.

Na Figura 2.8 temos os valores experimentais para o diâmetro do feixe do laser de $\mathrm{HeNe}$ em $632 \mathrm{~nm}$ utilizado em nossos experimentos como feixe de prova. A partir do ajuste obtivemos: $a=\left(0,3 \pm 0,9 \times 10^{-3}\right), \quad b=-(25,6 \pm 0,1) \times 10^{-3} \quad$ e

\footnotetext{
${ }^{1} \mathrm{O}$ parâmetro $\mathrm{z}_{\mathrm{c}}$ também é conhecido como comprimento de Rayleigh.
} 
$c=(5,97 \pm 0,02) \times 10^{-4}$. Utilizando estes dados encontramos a posição da cintura do laser de prova $(\mathrm{HeNe}) \mathrm{em} \mathrm{z}_{1}=\mathrm{z}_{\mathrm{p}}=(21,4 \pm 0,2) \mathrm{cm}$.

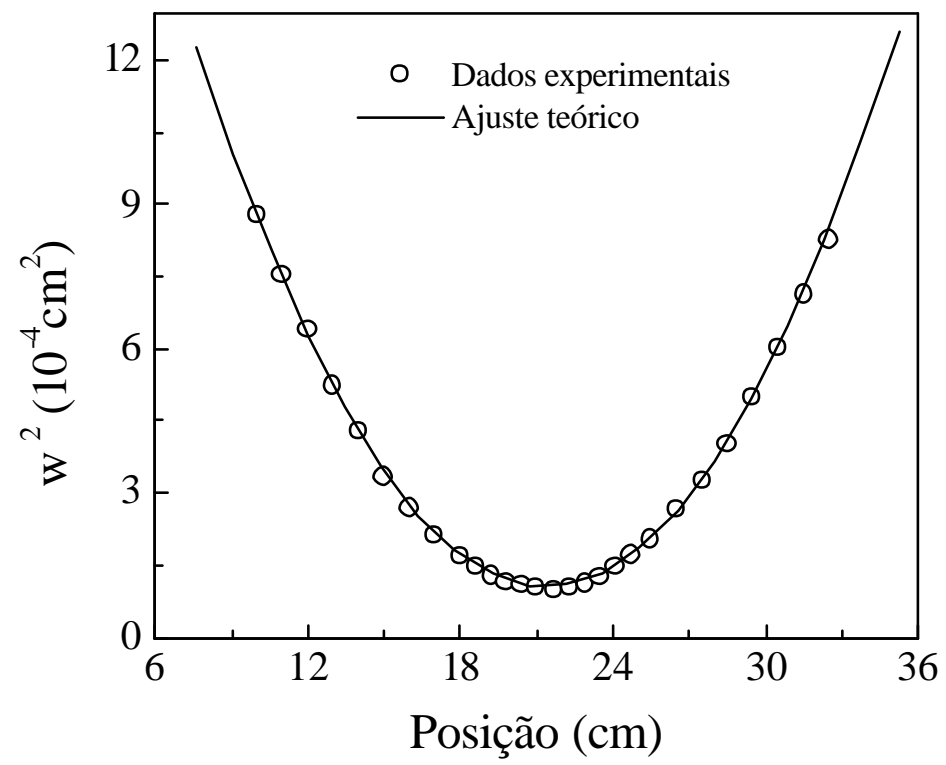

Figura 2.8 - Quadrado do diâmetro do feixe do laser de HeNe em função da distância da lente convergente de foco, $f=20 \mathrm{~cm}$. Os dados experimentais foram ajustados com a equação da parábola.

Para determinar $\mathrm{o}$ valor do parâmetro confocal do feixe, $\mathrm{z}_{\mathrm{c}}$, fizemos $\mathrm{o}$ seguinte procedimento: diminuímos dos valores das abscissas o valor da posição da cintura do laser, $\mathrm{z}_{1}$. Fizemos novamente $\mathrm{o}$ gráfico de $\mathrm{w}^{2}$ em função da posição (vide Figura 2.9) e ajustamos com a equação da parábola. Os novos valores das constantes foram: $\quad \mathrm{a}=\left(0,01 \pm 0,1 \times 10^{-3}\right), \quad \mathrm{b}=(8,2 \pm 0,1) \times 10^{-8} \quad \mathrm{e}$ $\mathrm{c}=(5,97 \pm 0,02) \times 10^{-4}$. De modo que fazendo $\mathrm{z}_{\mathrm{c}}=\sqrt{\mathrm{a} / \mathrm{c}}$, determinamos $\mathrm{o}$ parâmetro confocal do feixe. Para o feixe de prova $(\mathrm{HeNe}) \mathrm{z}_{\mathrm{cp}}=(4,1 \pm 0,2) \mathrm{cm}$. Usando este valor determinamos o diâmetro da cintura do feixe na posição de foco $\left(\mathrm{w}_{\mathrm{op}}=(9,1 \pm 0,2) \times 10^{-3} \mathrm{~cm}\right)$. Este mesmo procedimento foi usado para determinar $\mathrm{o}$ diâmetro do feixe de excitação $\mathrm{w}_{\mathrm{e}}$. Após determinar $\mathrm{w}_{\mathrm{e}} \mathrm{e} \mathrm{w}_{\mathrm{op}}$, calculamos através 
da Eq. (2.4) o parâmetro $m$ que é utilizado na equação de ajuste (Eq. (2.3)). Em nossas medidas trabalhamos com $\mathrm{V}=1,73$, onde se tem a otimização do sinal de LT (demonstrado por Sheldon e colaboradores [57]).

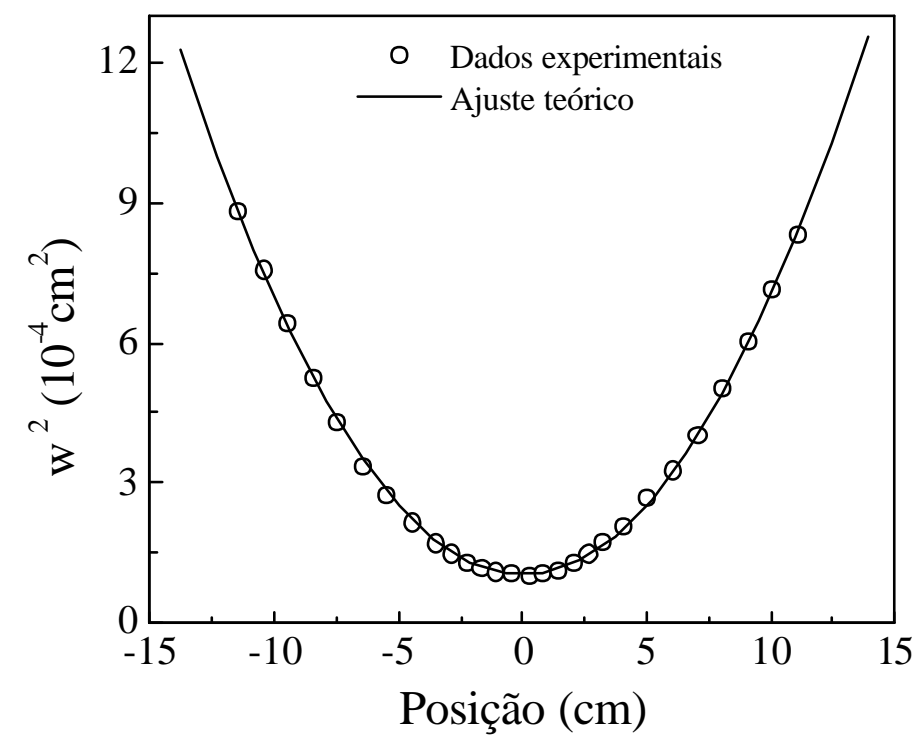

Figura 2.9 - Quadrado do diâmetro do feixe de prova ( HeNe) em 632nm, com o centro da parábola em $z=0$. Os dados experimentais foram ajustados com a equação da parábola.

Na Tabela 2.1 são apresentados os parâmetros utilizados nas medidas de LT, para os comprimentos de onda 632488514 e 808nm. É importante dizer que nós utilizamos em nossos experimentos vários comprimentos de onda, cujos dados não estão apresentados na tabela abaixo.

\begin{tabular}{|c|c|c|c|c|}
\hline$\lambda \quad(\mathrm{nm})$ & 632 & 488 & 514 & 810 \\
\hline$z_{c} \quad(\mathrm{~cm})$ & 4,1 & 1,1 & 0,75 & 0,56 \\
\hline $\mathrm{V}$ & 1,73 & 1,73 & 1,73 & 1,73 \\
\hline $\mathrm{w}_{\mathrm{e}}\left(10^{-3} \mathrm{~cm}\right)$ & - & 4,2 & 3,5 & 3,8 \\
\hline $\mathrm{w}_{\mathrm{p}}\left(10^{-3} \mathrm{~cm}\right)$ & 18,2 & - & - & - \\
\hline$m$ & - & 18,8 & 27,0 & 23,0 \\
\hline
\end{tabular}

Tabela 2.1 - Valores típicos dos parâmetros usados nos experimentos de LT. 


\subsection{Resultados}

É preferível que os materiais utilizados como meio ativo para laser de estado sólido possuam alta condutividade térmica, a fim de que seja possível ter um processo eficiente de dispersão do calor durante a operação laser, evitando desta forma aberrações óticas devido ao calor que possa ficar confinado no material.

A determinação da difusividade térmica foi realizada utilizando a técnica de LT. A Figura 2.10 mostra o transiente de LT excitando em 808nm a amostra de YAG dopada com $0,75 \%$ de $\mathrm{Nd}^{3+}$. Com o ajuste teórico a partir da Eq. (2.3), determinamos os parâmetros $\quad=-(2,400 \pm 0,005) \times 10^{-2} \mathrm{rad} \quad \mathrm{e}$ $\mathrm{t}_{\mathrm{c}}=(12,00 \pm 0,06) \times 10^{-2} \mathrm{~ms}$. Com o valor de $t_{c}$ e usando a equação $t_{c}=\mathrm{w}_{\mathrm{oe}}^{2} / 4 \mathrm{D}$, obtivemos $\mathrm{D}=(4,80 \pm 0,05) \times 10^{-2} \mathrm{~cm}^{2} \mathrm{~s}^{-1}$. Este valor está em bom acordo com o valor encontrado na literatura $\mathrm{D}=4,6 \times 10^{-2} \mathrm{~cm}^{2} \mathrm{~s}^{-1}$ [51]. Na Tabela 2.2 estão apresentados os resultados para todas as outras amostras estudadas.

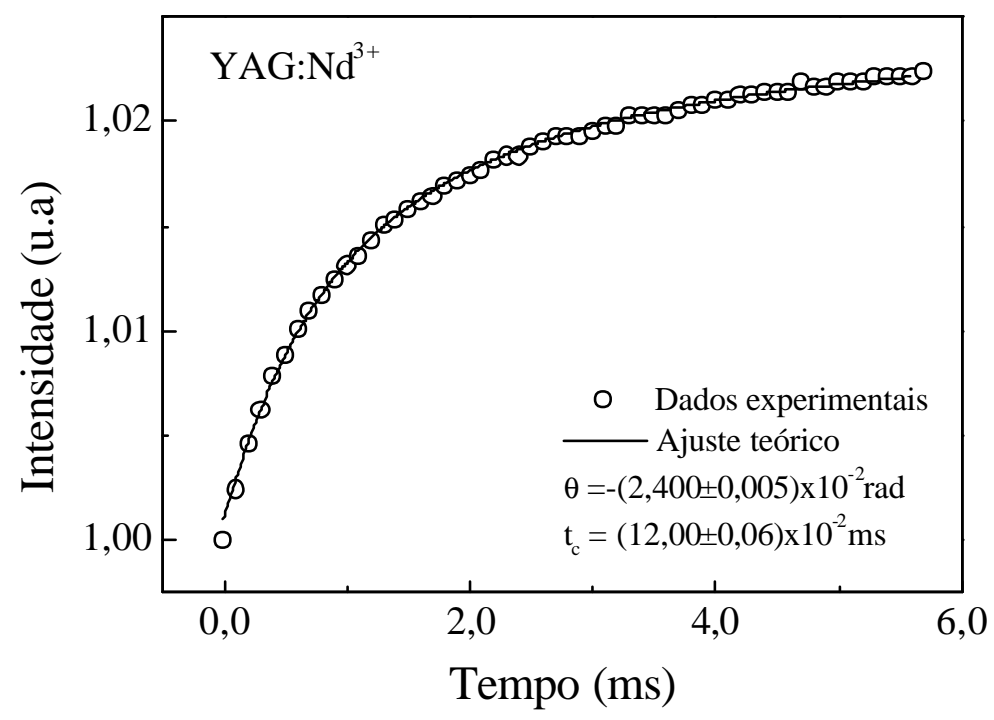

Figura 2.10 - Sinal do feixe de prova, ? ${ }_{p}=632 \mathrm{~nm}$, com $P=54 \mathrm{~mW} \lambda_{\text {exc }}=808 \mathrm{~nm}$. Ajuste teórico Eq. (2.3), com $V=1,73$ e $m=16,4$. 


\begin{tabular}{|c|c|c|c|c|}
\hline Amostra & $\begin{array}{c}? \mathrm{c} \\
\mathrm{J} / \mathrm{cm}^{3} \mathrm{~K}\end{array}$ & $\begin{array}{c}\mathrm{D} \\
10^{-3} \mathrm{~cm}^{2} / \mathrm{s} \\
\end{array}$ & $\begin{array}{c}\mathrm{k}_{\mathrm{Cal}} \\
10^{-3} \mathrm{~W} / \mathrm{Kcm}\end{array}$ & $\begin{array}{c}\mathrm{k}_{\mathrm{Lit}} \\
10^{-3} \mathrm{~W} / \mathrm{Kcm}\end{array}$ \\
\hline $\begin{array}{c}Y A G \\
\left(\mathrm{Y}_{3} \mathrm{Al}_{5} \mathrm{O}_{12}\right)\end{array}$ & $2,69^{\text {(a) }}$ & 48 & 129 & $130^{\text {(a) }}$ \\
\hline$Y V O_{4} / / c$ & $1,1^{\text {(a) }}$ & 48 & 52 & $52^{\text {(a) }}$ \\
\hline $\mathrm{YVO}_{4} \perp c$ & $1,1^{\text {(a) }}$ & 44 & 48,4 & $51^{(\mathrm{a})}$ \\
\hline $\begin{array}{c}B T O \\
\left(\mathrm{Bi}_{12} \mathrm{TiO}_{20}\right)\end{array}$ & $1,9^{\text {(b) }}$ & 6,8 & 12,9 & \\
\hline $\begin{array}{c}B S O \\
\left(\mathrm{Bi}_{12} \mathrm{SiO}_{20}\right)\end{array}$ & $2,5^{\text {(b) }}$ & 5,8 & 14,5 & \\
\hline $\begin{array}{l}\text { Fluorozirconato } \\
\qquad(Z B L A N)\end{array}$ & $2,96^{(\mathrm{c})}$ & $2,6^{(\mathrm{c})}$ & 7,7 & \\
\hline Fluoroindato ( $P G I Z C a)$ & $3,58^{(\mathrm{c})}$ & $2,9^{(\mathrm{c})}$ & 10,4 & \\
\hline Fluoroaluminato $(Y A B C)$ & $2,48^{(c)}$ & $3,3^{(\mathrm{c})}$ & 8,2 & \\
\hline $\begin{array}{l}\text { Aluminato } \\
(L S C A S)\end{array}$ & $2,82^{(\mathrm{d})}$ & $5,5^{(\mathrm{d})}$ & 15,5 & \\
\hline Soda lime & $2,04^{(\mathrm{e})}$ & $4,9^{(\mathrm{e})}$ & 10 & \\
\hline Calcohaleto & $1,5^{(\mathrm{f})}$ & $1,3^{(\mathrm{f})}$ & 1,9 & \\
\hline $\begin{array}{c}\text { Filme polimérico }(P P V) \\
(p \text {-fenileno-vinileno })\end{array}$ & 1,48 & 3,9 & 5,8 & \\
\hline
\end{tabular}

Tabela 2.2 - Valores de difusividade térmica (D). Sendo $\rho$ a densidade, c o calor específico, $k_{\text {Cal }}$ a condutividade térmica calculada, $k_{\text {Lit }}$ a condutividade térmica da literatura. (a) [61], (b) [62], (c) [7], (d) [16], (e) [6, 13], (f) [31].

Apesar dos cristais BTO e BSO pertencerem a mesma família dos silenitas, observamos uma diferença de $\sim 17 \%$ entre os valores obtidos para a difusividade térmica. Para o calcohalet o a difusividade térmica é aproximadamente a metade da difusividade dos vidros fluoretos e um quarto do vidro LSCAS . No cristal $\mathrm{YVO}_{4}$ foi observado uma pequena diferença no valor da difusividade com a orientação. Esta diferença é esperada, uma vez que, neste tipo de material o valor da difusividade depende da orientação [63-65]. 


\section{Capítulo 3}

\section{Eficiência Quântica.}

A eficiência quântica da fluorescência ? é uma das propriedades mais importantes de materiais óticos. Nas últimas duas décadas várias técnicas têm sido utilizadas para determinar o valor da eficiência quântica ?. Apesar de vários métodos terem sido desenvolvidos para determinar ?, a determinação precisa de seu valor, especialmente em amostras sólidas, tem sido muito difícil [16, 66, 67]. A distribuição espacial e espectral da luz emitida combinada com alguns processos, tal como reabsorção, reemissão, polarização, mudança no índice de refração e transferência de energia entre os íons, são limitações nos métodos aplicados para determinar ?, e pode ser a principal razão para os controvérsios resultados encontrados na literatura para este parâmetro.

\subsection{Medidas de Eficiência Quântica da Fluorescência.}

A eficiência quântica da fluorescência, ?, é definida como o número de fótons emitidos espontaneamente dividido pelo número de fótons de bombeio absorvidos. É comum expressar esta eficiência quântica através da relação dada por:

$$
?=\frac{\mathrm{W}_{\mathrm{R}}}{\mathrm{W}_{\mathrm{T}}}=\frac{\mathrm{t}_{\text {exp }}}{\mathrm{t}_{\mathrm{R}}}
$$


onde $\mathrm{W}_{\mathrm{R}}$ é a taxa de transição radiativa, $\mathrm{W}_{\mathrm{T}}$ é a taxa de decaimento total, $\tau_{\text {exp }}$ é o tempo de vida obtido experimentalmente e $t_{R}$ o tempo de vida puramente radiativo calculado pela teoria de Judd-Ofelt.

Vários fatores podem contribuir para o decaimento não radiativo de íons terras raras. Desta forma a taxa de decaimento total dada por $\mathrm{W}_{\mathrm{T}}=\mathrm{t}_{\text {exp }}^{-1}$, pode ser expressa como uma soma de várias contribuições [46]:

$$
\mathrm{W}_{\mathrm{T}}=\mathrm{W}_{\mathrm{R}}+\mathrm{W}_{\mathrm{mp}}+\mathrm{W}_{\mathrm{I}}+\mathrm{W}_{\mathrm{rc}}
$$

onde $\mathrm{W}_{\mathrm{mp}}$ representa $\mathrm{o}$ processo de decaimento por fônon (do inglês “multiphonon"), $\mathrm{W}_{\mathrm{I}}$ a transferência de energia para impurezas da matriz hospedeira e $\mathrm{W}_{\mathrm{rc}} \mathrm{o}$ processo de transferência de energia entre íons terras raras, normalmente atribuído a um processo de relaxação cruzada ("cross-relaxation").

(a) $\mathrm{Nd}-\mathrm{Nd}$

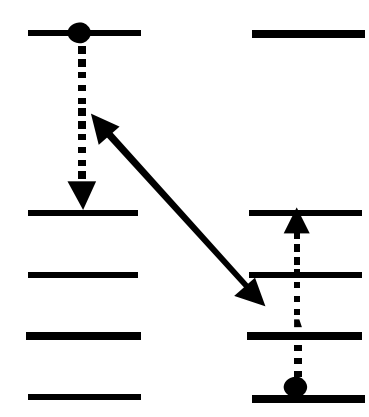

(c) Decaimento multifonon

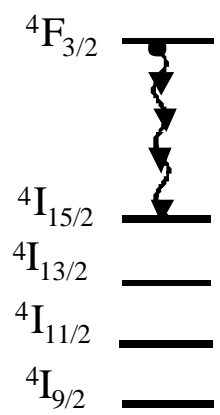

(b) $\mathrm{Nd}$ - ion metal
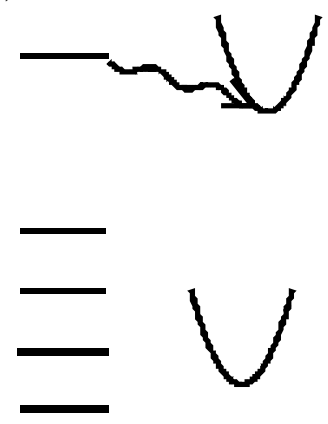

(d ) $\mathrm{Nd}-\mathrm{OH}^{-}$

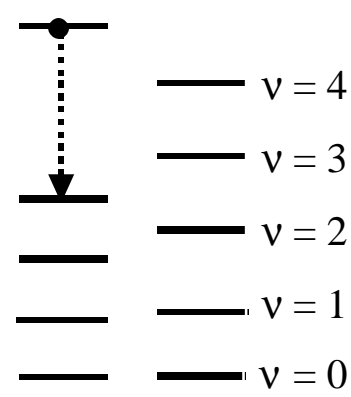

Figura 3.1 - Processos de "quenching" da fluorescência para o $\mathrm{Nd}^{3+}$. Figura tirada da Ref. [68]. 
$\mathrm{O}$ tempo de vida radiativo, $\mathrm{t}_{\mathrm{R}}$, é obtido através do método de Judd-Ofelt (JO). O cálculo é feito através das forças de oscilador obtidas a partir das várias linhas de absorção determinadas experimentalmente. Entretanto, deve-se lembrar que este método requer o conhecimento da concentração de íons, $\mathrm{N}_{0}$, de modo que há uma imprecisão no cálculo do tempo de vida, geralmente entre $10-15 \%$ [46].

No processo de relaxação cruzada $\left(\mathrm{W}_{\mathrm{cr}}\right)$, um íon no estado ${ }^{4} \mathrm{~F}_{3 / 2}$ relaxa não radiativamente para $\mathrm{o}{ }^{4} \mathrm{I}_{15 / 2}$ transferindo a energia para um outro íon $\mathrm{Nd}^{3+}$ inicialmente no estado fundamental (Figura 3.1 a). Na situação final, portanto, os dois íons encontram-se no mesmo estado ${ }^{4} \mathrm{I}_{15 / 2}$ e o resultado final é a conversão da energia de excitação em calor devido ao decaimento não radiativo em cascata até o nível fundamental.

Um outro processo que contribui para o decaimento não radiativo, é a transferência de energia para impureza (vide Figura 3.1 b e d). É sabido que no caso dos vidros fosfatos, o tempo de vida da fluorescência é fortemente afetado pela presença de $\mathrm{OH}^{-}$. Atualmente, se consegue controlar o processo de fabricação de vidros de tal forma a tornar desprezível o efeito de impurezas (água ou íons).

A taxa de relaxação por multi-fônons $\mathrm{W}_{\mathrm{mp}}$ depende fortemente do espectro dos fônons da matriz hospedeira, pois decresce exponencialmente com o número de fônons $q \approx ? E / \eta \omega$, onde ?E é diferença de energia entre o estado excitado e o nível inferior mais próximo e $\eta \omega$ é a energia de fônon. No caso do $\mathrm{Nd}^{3+}, ? \mathrm{E} \approx 5500 \mathrm{~cm}^{-1}$ entre os níveis ${ }^{4} \mathrm{~F}_{3 / 2}$ e ${ }^{4} \mathrm{I}_{15 / 2}$, vide Figura 3.1(c). Os vidros em geral apresentam energia de fônons entre 500 e $1000 \mathrm{~cm}^{-1}$. Por exemplo, o vidro fluoreto ZBLAN tem uma energia de fônon $\sim 580 \mathrm{~cm}^{-1}$ e o aluminato (LSCAS) $\sim 800 \mathrm{~cm}^{-1}$, o que 
corresponde a $q \sim 10$ e $q \sim 7$, respectivamente. Isto quer dizer que, no caso do vidro fluoreto, são necessários 10 fônons para ocorrer o decaimento não radiativo do nível ${ }^{4} \mathrm{~F}_{3 / 2} \rightarrow{ }^{4} \mathrm{I}_{15 / 2}$, o que é um número muito grande. Na grande maioria dos vidros (com exceção dos vidros boratos) o efeito de $\mathrm{W}_{\mathrm{mp}}$ é despresível [46].

Tanto em vidros quanto em cristais, tem-se observado que $t_{\text {exp }}$ decresce com o aumento da concentração, vide Figura 3.2. Este comportamento pode ser descrito pela seguinte expressão empírica [69]:

$$
\mathrm{t}_{\text {exp }}=\frac{\mathrm{t}_{0}}{1+\left(\mathrm{N}_{0} / \mathrm{N}_{\mathrm{cq}}\right)^{\mathrm{p}}}
$$

onde $\mathrm{t}_{0}$ é o tempo de vida experimental no limite de concentração zero, $\mathrm{N}_{0}$ (íons $/ \mathrm{cm}^{3}$ ) é a concentração de íons, $\mathrm{N}_{c q}$ (íons $/ \mathrm{cm}^{3}$ ) é a concentração de supressão crítica (“quenching”), na qual o tempo de vida experimental é reduzido pela metade. O parâmetro $p$ indica o número de íons envolvidos no mecanismo de supressão.

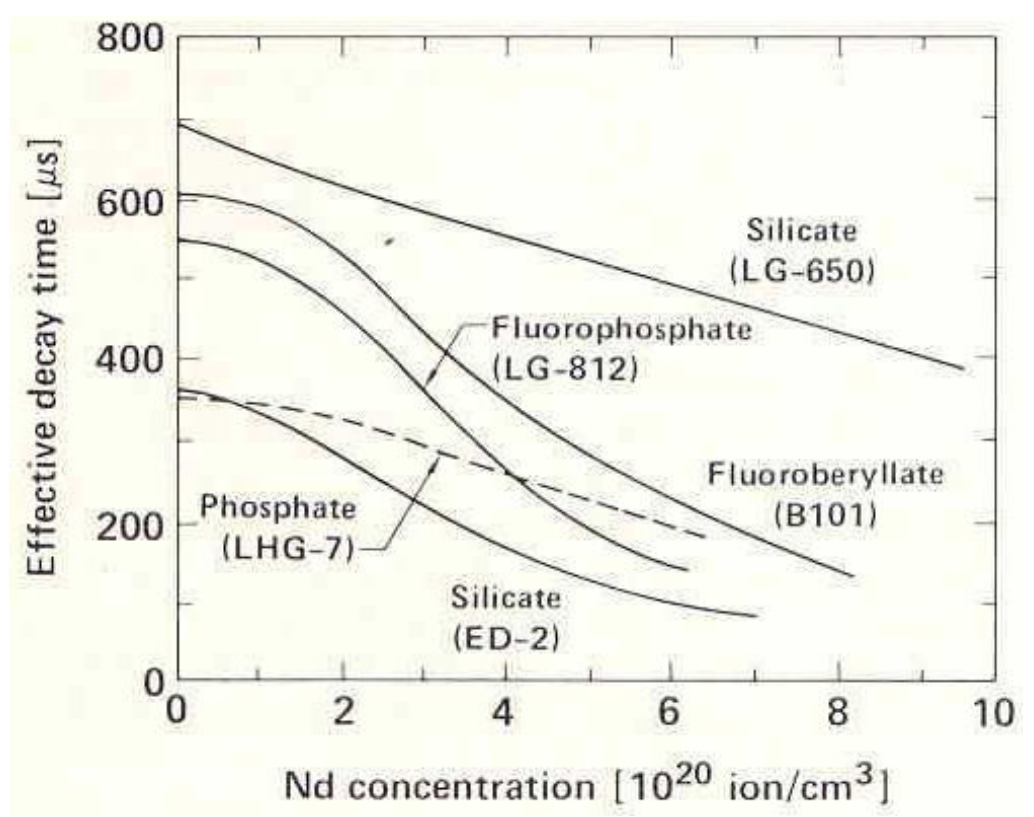

Figura 3.2 - Tempo de vida em função da concentração. Figura tirada da Ref. [68]. 
Observa-se que para um mesmo íon (no nosso caso o $\mathrm{Nd}^{3+}$ ) $\mathrm{N}_{\mathrm{cq}}$ varia muito com a matriz. Na Tabela 3.1 estão listados vários valores da concentração de “quenching” para vários vidros dopados com $\mathrm{Nd}^{3+}$ encontrados na literatura.

\begin{tabular}{ccc}
\hline \hline Tipo de vidro & $\begin{array}{c}\mathrm{N}_{\mathrm{cq}} \\
\left(10^{20} \mathrm{~cm}^{-3}\right)\end{array}$ & Ref. \\
\hline \hline Silicato & $3,9-6,0$ & {$[46]$} \\
Fosfato & $3,9-8,6$ & {$[46]$} \\
Fluorofosfato & $3,0-4,0$ & {$[46]$} \\
$\begin{array}{c}\text { Fluorozirconato } \\
\text { (ZBLAN) }\end{array}$ & 7,9 & {$[70]$} \\
$\begin{array}{c}\text { Aluminato } \\
\text { (LSCAS) }\end{array}$ & 2,3 & {$[71]$} \\
\hline
\end{tabular}

Tabela 3.1 - Valores da concentração de “quenching” para o $\mathrm{Nd}^{3+}$.

Balda e colaboradores [72] estudaram a dependência do tempo de vida com a concentração de $\mathrm{Nd}^{3+}$ em vários tipos de vidros fluoretos (ZBLAN, BIGaZYTZr e PZG ) tal como mostra a Figura 3.3. Eles compararam o tempo de vida radiativo $t_{R}$, obtido pelo método de Judd-Ofelt com o tempo de vida experimental, $\mathrm{t}_{\text {exp }}$, para determinar a eficiência quântica ? pela razão $t_{\exp } / t_{R}$. Nós usamos a expressão (3.3) para ajustar os resultados de Balda, tal como mostra a Figura 3.2, e determinamos $\tau_{0}$ e $\mathrm{N}_{c q}$ (vide Tabela 3.2). É esperado que $\tau_{0}$ seja aproximadamente igual ao tempo de vida radiativo $t_{R}$. A diferença observada entre $\tau_{0}$ e $t_{R}$, pode ser explicada considerando que no cálculo de JO tem uma imprecisão intrínseca de $10-15 \%$. 

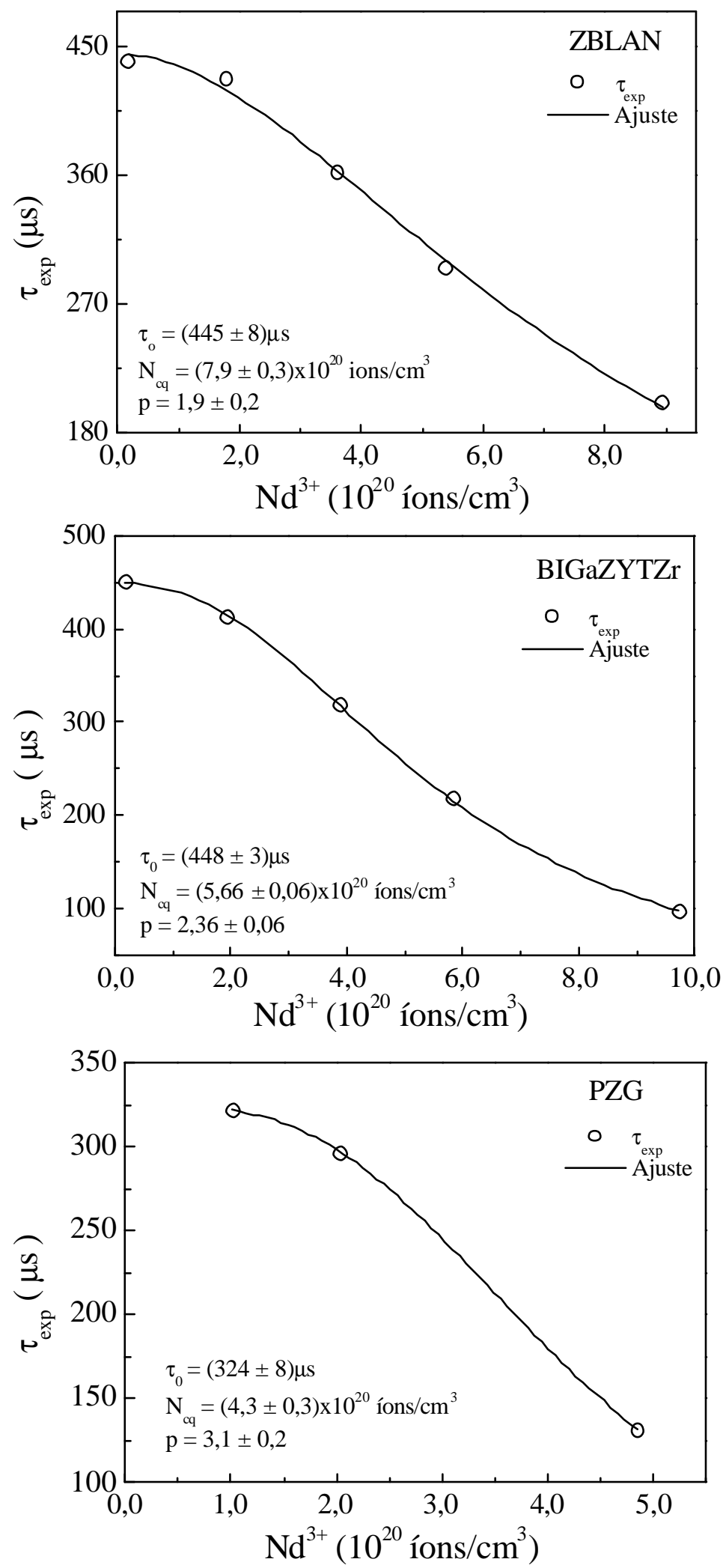

Figura 3.3 - Tempo de vida experimental, $\tau_{\text {exp }}$ em função da concentração [72]. Ajuste teórico usando a expressão (3.3). 


\begin{tabular}{|c|c|c|c|c|}
\hline Vidros & $\begin{array}{c}\mathrm{N}_{\mathrm{cq}} \\
\left(10^{20} \text { íns } / \mathrm{cm}^{3}\right)\end{array}$ & $\begin{array}{c}\mathrm{t}_{0} \\
(\mu s)\end{array}$ & $\begin{array}{l}\mathrm{t}_{\mathrm{R}} \\
(\mu \mathrm{s})\end{array}$ & $?_{\mathrm{JO}}$ \\
\hline $\begin{array}{c}\text { Fluorozirconato } \\
\qquad(Z B L A N)\end{array}$ & 7,9 & 445 & 499 & 0,89 \\
\hline $\begin{array}{l}\text { Fluoroindato } \\
(B I G a Z Y T Z r)\end{array}$ & 5,7 & 448 & 537 & 0,83 \\
\hline $\begin{array}{l}\text { Fluoroindato } \\
\qquad(P Z G)\end{array}$ & 4,3 & 324 & 375 & 0,86 \\
\hline $\begin{array}{c}\text { Fosfato } \\
(L G-750)\end{array}$ & 6,87 & 366 & 352 & 1,04 \\
\hline
\end{tabular}

Tabela 3.2 - Tabela comparando a concentração cúbica de supressão $\left(N_{c q}\right), ?_{J O}$ eficiência quânticao, tempo de vida de baixa concentração $t_{0}$ (obtido através da Eq. (3.3)) e o tempo de vida radiativo, $t_{R}$, calculado pelo método de JO.

Há várias dificuldades envolvidas na determinação experimental da eficiência quântica, $\eta$. Dentre os métodos experimentais mais utilizados para determinar $\eta$, podemos citar o método da esfera integradora cuja desvantagem é a necessidade de calibração do detetor e imprecisão nos resultados obtidos, uma vez que a luz da fluorescência é policromática. O método da fluorescência, cuja dependência com o tempo de vida e o decaimento da fluorescência, torna-o inadequado para sistemas de energia de multi-níveis, uma vez que o comportamento destes parâmetros não obedecem uma forma exponencial. A idéia fundamental dos métodos fototérmicos, é a detecção dos processos não radiativos, ou seja, determinar a fração da energia que é absorvida e convertida em calor devidos aos processos não radiativos. Nesta área, a espectroscopia fotoacústica foi um dos métodos pioneiros na determinação da eficiência quântica da fluorescência de sólidos [73]. Desde então estes métodos têm sido aplicados para determinar a eficiência quântica da fluorescência de líquidos [74], polímeros [75], vidros [16, 66] e cristais [76, 77]. 
Outro método utilizado para determinar a eficiência quântica da fluorescência é o método de LT. Este método foi introduzido por Brannon e Magde em 1979 [78]. Em 1982 ele foi usado para determinar a eficiência quântica, $\eta$, de soluções fluorescentes por Lesiecki e Drake [75] e em 1989 em polímeros por Shen e Snook [74]. Em nosso grupo, este método foi utilizado pela primeira vez em 1998 para determinar o valor absoluto de $\eta$ em sólidos dopados [16].

Devido a dificuldade experimental e incerteza dos métodos tradicionais, existem poucas medidas absolutas de eficiência quântica na literatura. Também são poucos os trabalhos que relatam medidas da eficiência quântica em função da concentração de dopante. A Figura 3.4 e Figura 3.5 mostra valores da eficiência quântica dos vidro silicato $\mathrm{ED}-2$ e do fluoreto $\mathrm{LG}-750$ dopados com $\mathrm{Nd}^{3+}$. Na Figura 3.6 temos resultados de eficiência quântica no YAG. Como podemos observar, existe muitos resultados controversos.

No caso do YAG, cálculos de Judd-Ofelt e tempo de vida [79] indica que uma amostra com $1 \%$ de $\mathrm{Nd}^{3+}$ pode ter uma eficiência quântica de $\sim 0,91$. Medidas de luminescência por Dianov e colaboradores [80], um experimento de refrigeração ótica por Kushida e Geusic [81] e estudos dos tempos de vida com a temperatura por Liao e Weber [82] concordam com esta previsão e dão valores de ? que varia de 0,98-0,88. O resultado obtido por Rosencwaig e Edward [66] $(?=0,97 \pm 0,02)$ para uma amostra com 1,2\% (vide Figura 3.6), também corrobora com estes valores. 


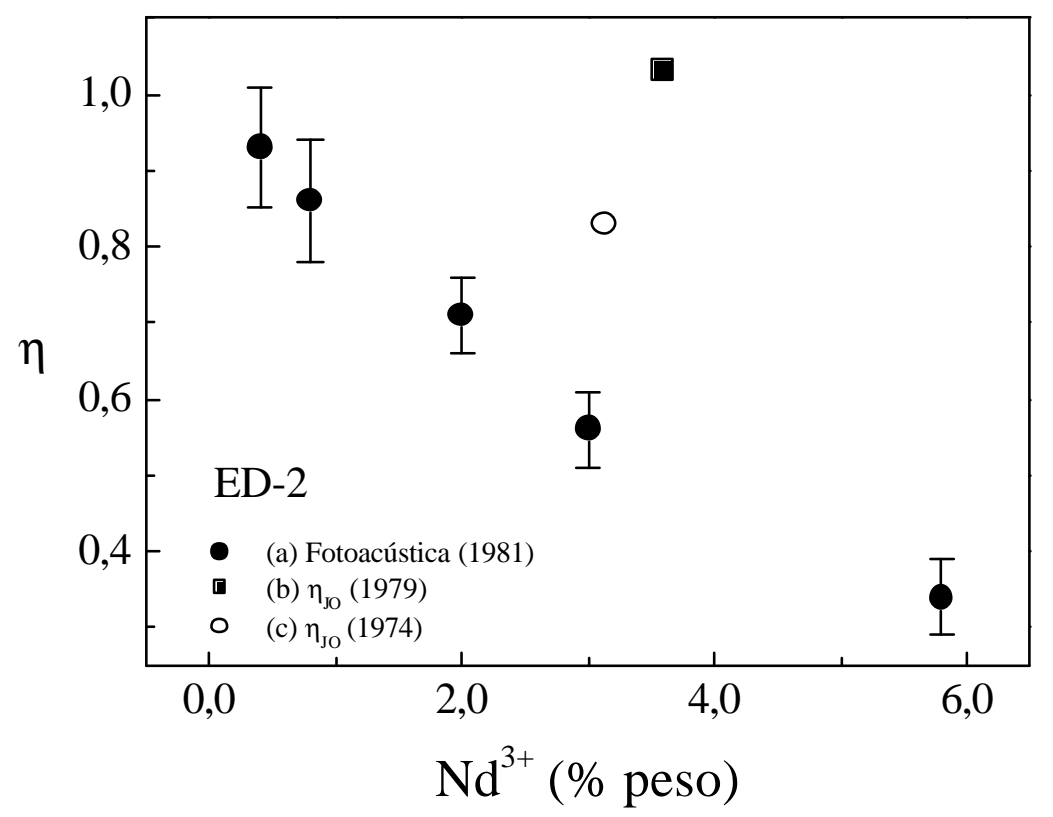

Figura 3.4 - Eficiência quântica do vidro silicato ED-2:Nd ${ }^{3+}$ em função da concentração. Os dados de (a) foram obtidos por Rosencwaig [66] por método fotoacústico, (b) pelo método de Judd-Ofelt por Linford e colaboradores [83] com $t_{R}=359 \mu \mathrm{s}$ e (c)pelo método de Judd-Ofelt por Krupke [84] com $t_{R}=372 \mu \mathrm{s}$.

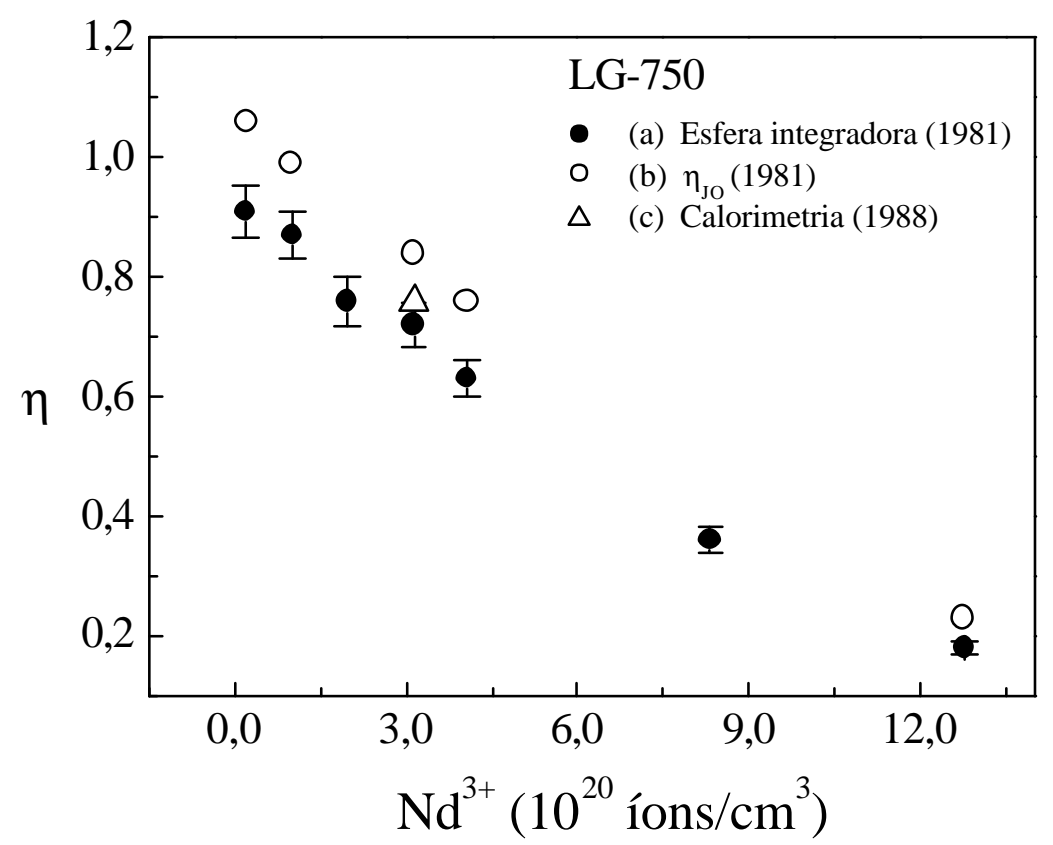

Figura 3.5 - Eficiência quântica do vidro fosfato LG-750:Nd ${ }^{3+}$ em função da concentração. Os resultados de (a) e (b) foram determinados por Caird e colaboradores [85] utilizando o método da esfera integradora e o método de Judd-Ofelt com $t_{R}=352 \mu \mathrm{s}$, respectivamente. $O$ resultado indicado por (c) foi obtido por Ramponi [86] utilizando o método de calorimetria. 


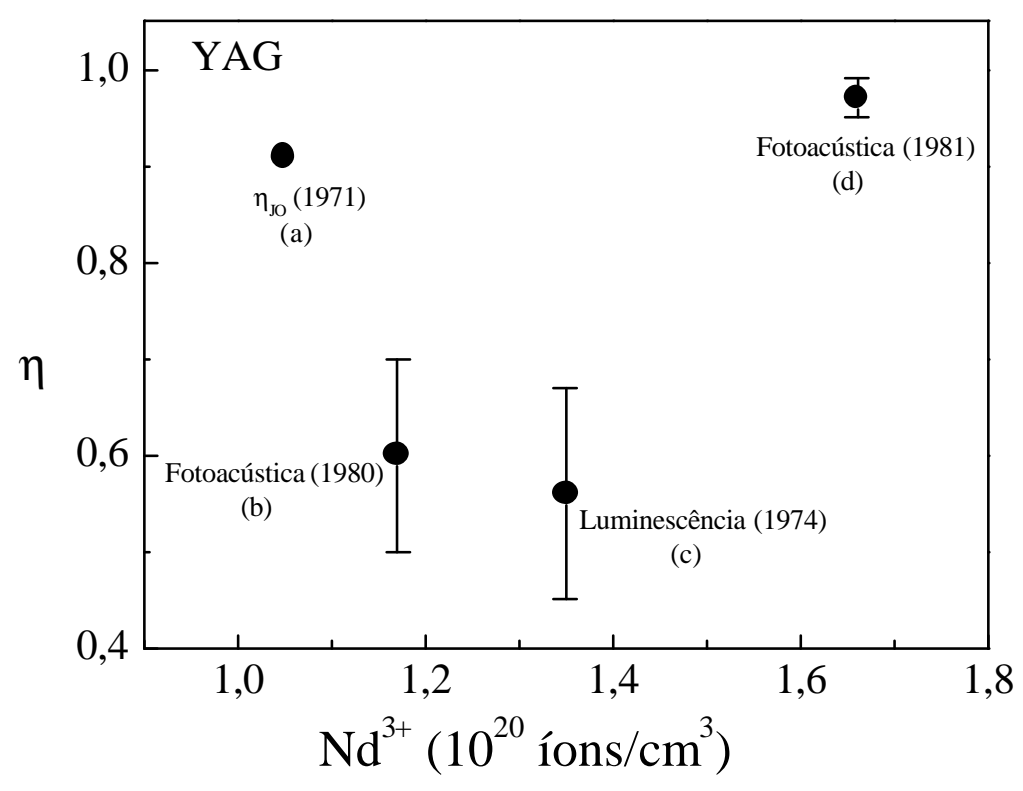

Figura 3.6 - Eficiência quântica do YAG: $\mathrm{Nd}^{3+}$ em função da concentração obtida por vários métodos. (a) Krupke [79] com $t_{R}=259 \mu \mathrm{s}$; (b) Powell e colaboradores [87], (c) Singh e colaboradores [88] e (d) Rosencwaig e Edward [66].

\subsection{Medidas de eficiência quântica com a técnica de LT}

Neste item iremos apresentar os resultados de eficiência quântica obtidos por meio da técnica de LT. As medidas foram realizadas no vidro silicato LSCAS e nos vidros fluoretos YABC, ZBLAN, PGIZCa .

É importante relembrar que o sinal de LT é proporcional a ? $=\mathrm{TPAL}_{\text {eff }}$, dado por (Eq. (2.5))

$$
\mathrm{T}=\left(\frac{?}{\mathrm{PAL}_{\mathrm{eff}}}\right)=\left[\frac{1}{\mathrm{k} ?_{\mathrm{p}}} \frac{\mathrm{ds}}{\mathrm{dT}}\right] \varphi
$$

Como vimos no Capítulo 1, em sólidos dopados com $\mathrm{Nd}^{3+}$, geralmente todos os níveis de energia excitados com energia maior do que o nível ${ }^{4} \mathrm{~F}_{3 / 2}$ decaem não radiativamente em forma de cascata, com emissão de fônons, para o nível metaestável ${ }^{4} \mathrm{~F}_{3 / 2}$ (Figura 3.7). A partir deste nível a fluorescência pode ser gerada. 
A frequiência média da emissão pode ser obtida através da relação $\left\langle ?_{\text {em }}\right\rangle=\sum \beta_{i} ?_{i}$, onde $\beta_{i}$ são as razões de ramificação ou "branching ratios". Este parâmetro mede a relação entre as probabilidades das transições eletrônicas que ocorrem a partir do mesmo nível, neste caso o nível ${ }^{4} \mathrm{~F}_{3 / 2}$ como pode ser visto na Figura 3.7.

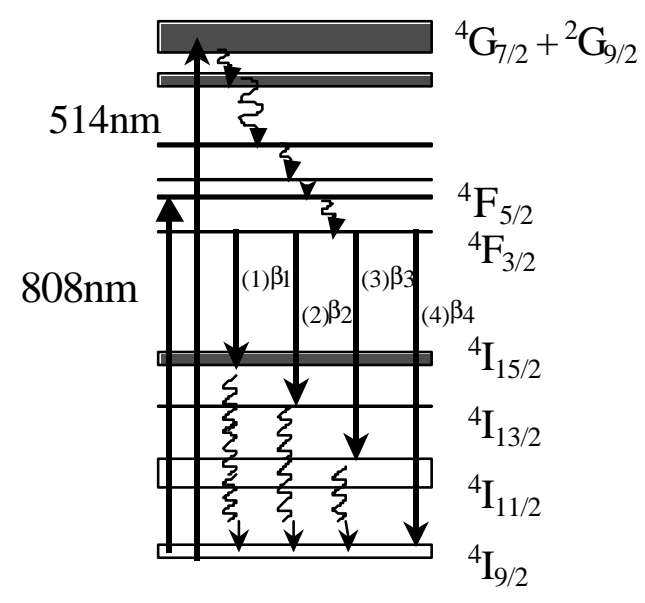

Figura 3.7 - Diagrama parcial dos níveis de energia do $\mathrm{Nd}^{3+}$.

Como a medida de LT é feita de maneira a determinar o valor de $\varphi$, o valor de ? pode ser obtido pela equação $\varphi=1-?\left\langle ?_{\mathrm{em}}\right\rangle / ?_{\mathrm{exc}}$ (Eq. (2.6)) com o prévio conhecimento do valor de $\left\langle ?_{\mathrm{em}}\right\rangle$. Esta é a idéia básica para determinar ? por qualquer método fototérmico. Em princípio, $\varphi$ pode ser determinado a partir de $\theta$ se o fator $\mathrm{k}^{-1} \mathrm{ds} / \mathrm{dT}$ for conhecido. Porém, $\mathrm{k}^{-1} \mathrm{ds} / \mathrm{dT}$ geralmente não é encontrado na literatura. No nosso primeiro trabalho utilizamos uma amostra como referência que apresenta fluorescência desprezível, consequentemente $?=0$ e $\varphi_{\mathrm{r}}=1$. No caso de amostras com baixa concentração de dopante, suas propriedades físicas (particularmente $\mathrm{k}$ de $\mathrm{ds} / \mathrm{dT}$ ) são similares aos valores dos sólidos não dopados (a matriz hospedeira) [16]. Portanto comparando os dados da amostra dopada $\left(\mathrm{T}_{\mathrm{d}}\right)$ com $\mathrm{a}$ amostra referencia $\left(\mathrm{T}_{\mathrm{r}}\right)$, Eq. (3.4), temos 


$$
\frac{\mathrm{T}_{\mathrm{Nd}}}{\mathrm{T}_{\mathrm{r}}}=\varphi_{\mathrm{Nd}}=1-?\left\langle ?_{\mathrm{em}}^{-1}\right\rangle ?_{\mathrm{ex}}
$$

Pela lei de Lambert-Beer, $\mathrm{T}=[1-\mathrm{R}]^{2} \mathrm{e}^{-\mathrm{AL}}$, podemos estimar o quanto de luz é transmitida por uma amostra, onde $\mathrm{R}$ é o coeficiente de reflexão, A o coeficiente de absorção e L a espessura da amostra. De acordo com essa expressão, por exemplo, o vidro aluminato (LSCAS) com $0 \%$ de $\quad \mathrm{Nd}^{3+} \quad$ (não dopada), $\mathrm{L}=0,36 \mathrm{~cm}, \quad \mathrm{~A}=0,07 \mathrm{~cm}^{-1} \quad$ em $\quad ?=810 \mathrm{~nm} \quad$ e $\mathrm{R}=0,06$, apresenta uma transmitância de $\mathrm{T} \approx 86 \%$. Ou seja, quase toda luz incidente é transmitida pela amostra. Este exemplo ilustra que a principal dificuldade deste método, em amostras não dopadas, está em determinar o coeficiente de absorção da amostra referência, que é um parâmetro crucial na normalização de $T_{r}$. A precisão da medida de $A$ é pequena se $\mathrm{AL}<<1$. Quanto maior for a transparência da amostra, maior será o erro na determinação da eficiência quântica.

Na Figura 3.8 temos os resultados de LT no aluminato (LSCAS ) em função da potência de excitação, para várias amostras com diferentes concentrações de $\mathrm{Nd}^{3+}(0$ a 4,5\%). As medidas foram realizadas excitando com um laser de Ti:safira sintonizado em $810 \mathrm{~nm}$. A vantagem de excitar nesta linha, é que ela que corresponde ao comprimento de onda máximo do pico de absorção do nível ${ }^{4} \mathrm{~F}_{5 / 2}$ (Figura 3.7). Como a amplitude do sinal de LT é proporcional a absorção, erros na determinação do parâmetro $\mathrm{T}$ podem ser minimizados.

Nós ajustamos os dados da Figura 3.8 com uma função linear, $? / \mathrm{L}_{\text {eff }}=\mathrm{a}+\mathrm{bP}$, obtendo as constantes $a$ e $b$ para cada amostra. Desde que $\mathrm{b}=$ ?/PL $\mathrm{effi}$, normalizando pelo coeficiente de absorção, A, nós obtemos o parâmetro $\mathrm{T}_{\mathrm{Nd}}$ para cada amostra, cujos resultados estão apresentados na Figura 3.9. 
Usando o parâmetro $\mathrm{T}_{\mathrm{r}}=11,54 \mathrm{~W}^{-1}$ da amostra base, nós calculamos a fração da energia absorvida e convertida em calor para cada amostra, $\varphi$, respectivamente. A eficiência quântica da fluorescência, $\eta$, é então obtida pela Eq. (3.5) usando o valor $?_{\text {exc }}\left\langle ?_{\text {em }}^{-1}\right\rangle=0,767$, onde $\left\langle ?_{\text {em }}^{-1}\right\rangle=0,949 \mu \mathrm{m}^{-1}[16]$

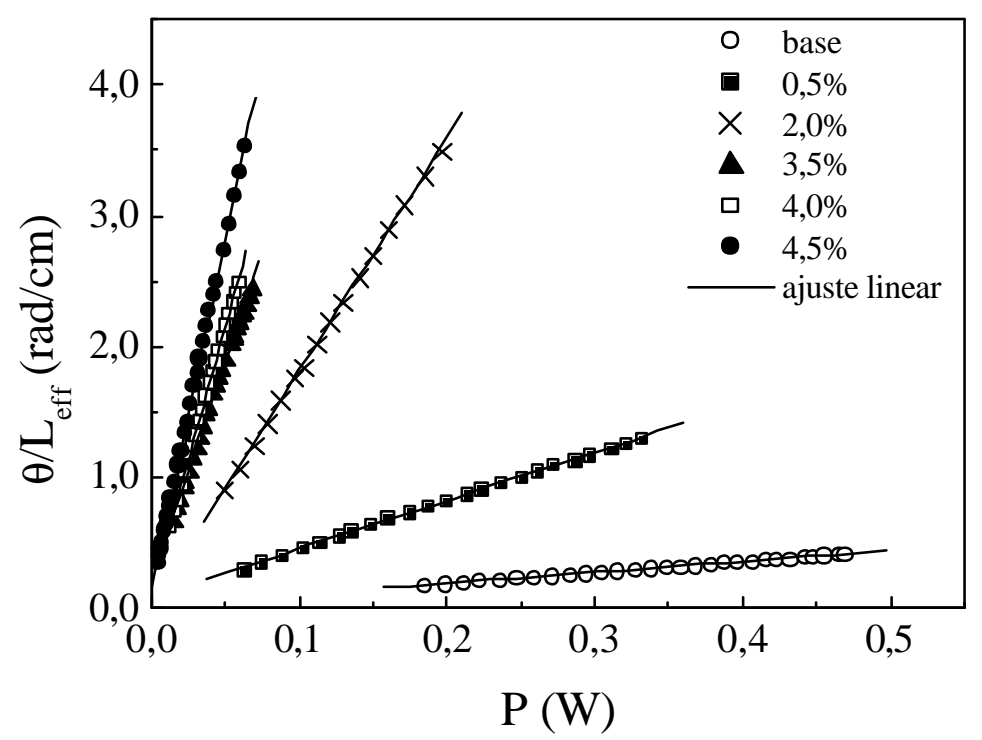

Figura 3.8 - Sinal de lente térmica do vidro aluminato de cálcio (LSCAS), para diferentes valores da potência de excitação do laser em $810 \mathrm{~nm}$. Comprimento de onda do feixe de prova $?_{p}=632 \mathrm{~nm}$. A linha sólida é o ajuste linear.

Estas mesmas amostras já haviam sido estudadas pelo nosso grupo em 1998 excitando em 514nm [16]. Porém, neste trabalho anterior cometemos um erro na análise dos resultados. Em geral, nos artigos de LT, na Eq. (3.4), é usado a AL ao invés de $\mathrm{AL}_{\text {eff }}$. Para baixo coeficiente de absorção onde $\mathrm{AL}<0,1$, é válida a aproximação $\mathrm{L} \approx \mathrm{L}_{\text {eff }}$. No entanto, esta aproximação não é válida nas amostras que estudamos, levando ao erro mencionado acima. Nós refizemos os cálculos da eficiência quântica considerando agora o termo $\mathrm{AL}_{\text {eff }}$. Os resultados podem ser vistos na Figura 3.10. Nesta figura temos também o comportamento da eficiência 
quântica em função da concentração de $\mathrm{Nd}^{3+}$. Podemos verificar ainda que os resultados obtidos tanto em $810 \mathrm{~nm}$ como em $514 \mathrm{~nm}$ estão em bom acordo.

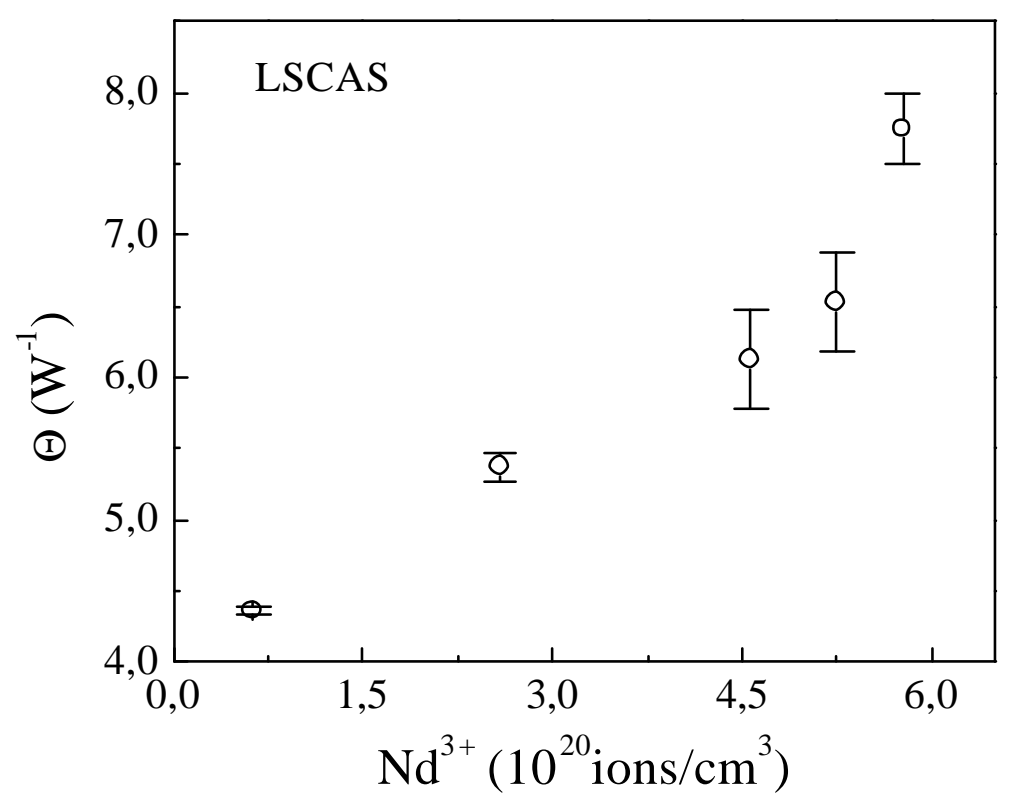

Figura 3.9 - Sinal de lente térmica normalizado versus a concentração de $\mathrm{Nd}^{3+}$ para o vidro aluminato (LSCAS) em $?_{\mathrm{exc}}=810 \mathrm{~nm}$.

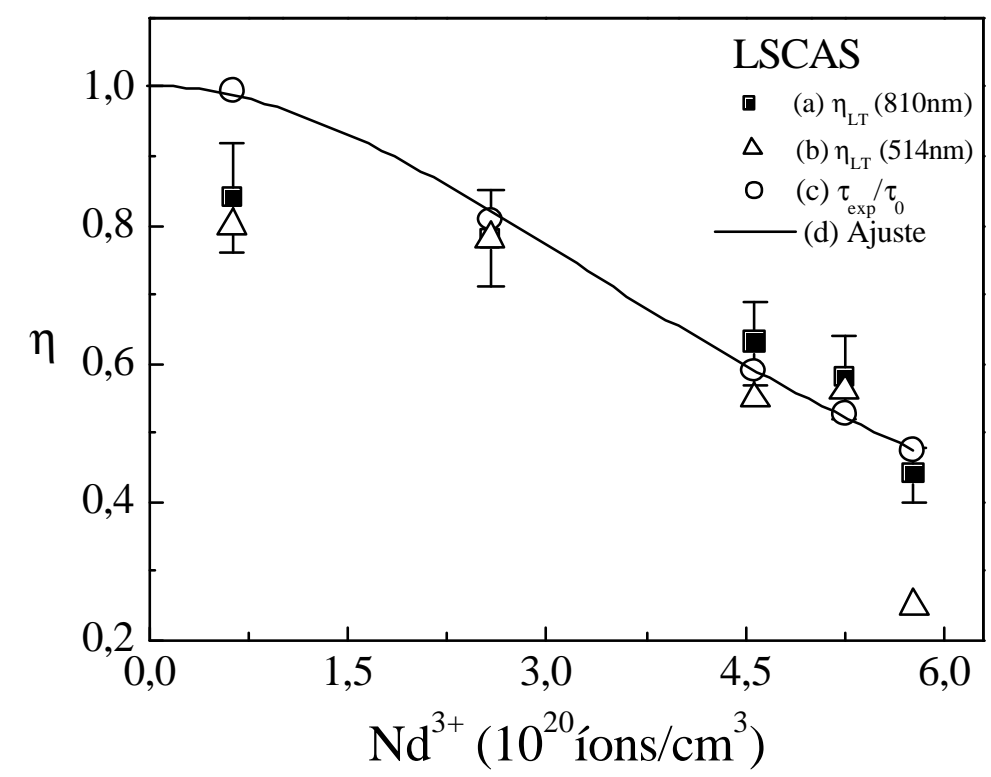

Figura 3.10 - Eficiência quântica do vidro aluminato (LSCAS) em função da concentração de $\mathrm{Nd}^{3+}$. (a) Resultado de LT em ? exc $=810 \mathrm{~nm} ;$ (b) resultado em $?_{\text {exc }}=514 \mathrm{~nm} ;($ c) resultado obtido pelo método de Judd-Ofelt e (d) ajuste teórico usando a Eq. (3.3) obtendo $N_{c q}=(5,48 \pm 0,05) \times 10^{20}$ ions $/ \mathrm{cm}^{3}, p=2$ e $t_{0}=(322 \pm 2) \mu \mathrm{s}$. 
Utilizando a mesma metodologia, nós determinamos a eficiência quântica nos vidros fluoretos ZBLAN e PGIZCa, excitando em $797 \mathrm{~nm}$, comprimento de onda que corresponde ao pico máximo da absorção do nível ${ }^{4} \mathrm{~F}_{3 / 2}$ destes vidros. Os resultados estão apresentados na Figura 3.11 e Figura 3.12. Nestas amostras, devido a grande dificuldade em determinar o coeficiente de absorção da amostra referencia (amostra pura), nós utilizamos como referência matrizes dopadas com baixa concentração de $\mathrm{Co}^{2+}$. Neste caso, é conhecido que matriz dopada com este íon a fluorescência é desprezível, tal que $\varphi \sim 1(? \approx 0)$ [7].

O decréscimo do tempo de vida experimental com a concentração, Figura 3.10, Figura 3.11 e Figura 3.12, foi ajustado com a expressão empírica Eq. (3.3), de onde determinamos o tempo de vida no limite de baixa concentração $t_{0}$ e $a$ concentração de "quenching" $\mathrm{N}_{\mathrm{cq}}$. $\mathrm{O}$ resultados podem ser vistos na Tabela 3.3. Como podemos notar, o tempo de vida $\mathrm{t}_{0}$ obtido do ajuste é bem próximo do tempo de vida radiativo $t_{R}$ obtido pelo método de Judd-Ofelt.

Nossos resultados de tempo de vida, Figura 3.10, Figura 3.11 e Figura 3.12, decrescem com a concentração similarmente aos dados de Balda e colaboradores [72]. Como as nossas amostras foram preparadas em atmosfera seca, não podemos atribuir o decaimento não radiativo observado devido à presença de água. E levando em conta o fato deles apresentarem baixa freqüência de fônon (aluminato $\sim 800 \mathrm{~cm}^{-1}$ e fluoretos $\sim 500 \mathrm{~cm}^{-1}$ ), a probabilidade de decaimento não radiativo do nível ${ }^{4} \mathrm{~F}_{3 / 2} \rightarrow{ }^{4} \mathrm{I}_{15 / 2}$ pode ser desprezada e valores de ? $\sim 1$ são esperados no regime de baixa concentração. 
A Figura 3.13 mostra o comportamento de ? com a concentração de $\mathrm{Nd}^{3+}$ para as quatro amostras estudadas e juntamente os resultados do vidro calcogeneto GaLaS obtidos no grupo [43]. Para o intervalo de concentração $0,5-1,0$ mol \% de $\mathrm{Nd}^{3+}$, os valores obtidos de ? variaram entre $0,76-1,0$ com um erro experimental de aproximadamente $10 \%$. Desde que $?=\mathrm{t}_{\exp } / \mathrm{t}_{\mathrm{R}}$, nossos resultados mostram que ? se aproxima da unidade para todas as amostras com baixa concentração de dopante, com exceção do LSCAS, onde a eficiência quântica obtida pelo método de JO é aproximadamente $17 \%$ maior do que a eficiência quântica obtida pela LT. Na Tabela 3.4 sumarizamos os resultados obtidos neste item.

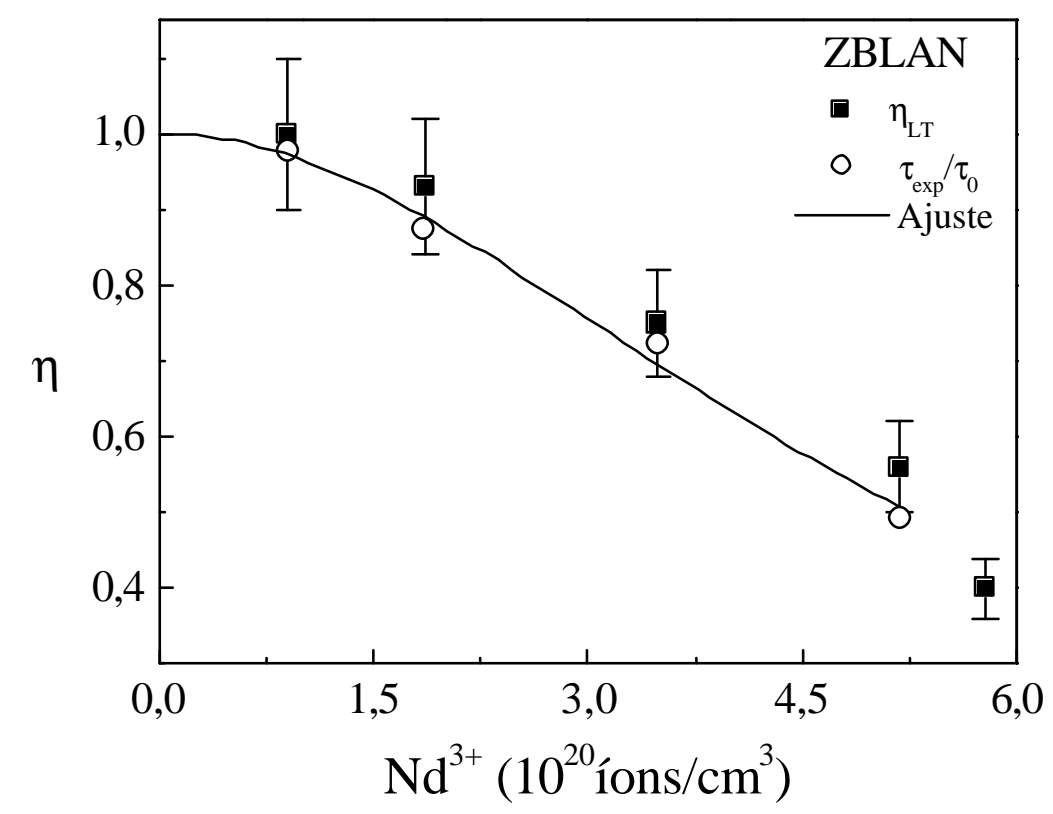

Figura 3.11 - Eficiência quântica do vidro fluoreto ZBLAN. ? ${ }_{\text {exc }}=797 \mathrm{~nm}$. $N_{c q}=(5,3 \pm 0,2) \times 10^{20}$ ions $/ \mathrm{cm}^{3}, p=2$ e $t_{0}=(527 \pm 11) \mu \mathrm{s}$. 


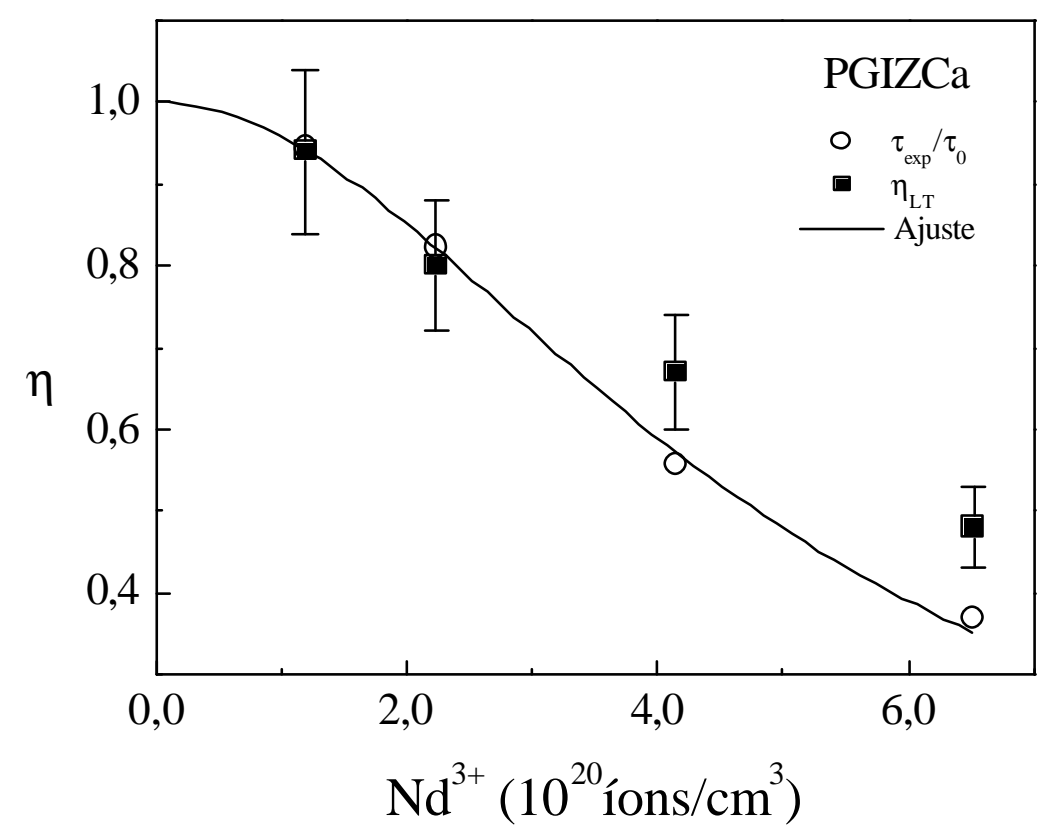

Figura 3.12 - Eficiência quântica do vidro fluoreto PGIZCa. $\lambda_{\text {exc }}=797 \mathrm{~nm}$. $N_{c q}=(4,8 \pm 0,2) \times 10^{20}$ ions $/ \mathrm{cm}^{3}, p=2$ e $t_{0}=(446 \pm 8) \mu \mathrm{s}$.

\begin{tabular}{cccccc}
\hline \hline Vidros & $\begin{array}{c}\mathrm{N}_{\mathrm{cq}} \\
\left(10^{20} \text { ions } / \mathrm{cm}^{3}\right)\end{array}$ & $\begin{array}{c}\mathrm{t}_{0} \\
(\mu s)\end{array}$ & $\begin{array}{c}\mathrm{t}_{\mathrm{R}} \\
(\mu s)\end{array}$ & $?_{\mathrm{JO}}$ & $?_{0}$ \\
\hline \hline $\begin{array}{c}\text { Aluminato } \\
(L S C A S)\end{array}$ & 5,48 & 322 & $320^{(\mathrm{a})}$ & 1,0 & 0,85 \\
$\begin{array}{c}\text { Fluorozirconato } \\
(\text { ZBLAN })\end{array}$ & 5,3 & 527 & $499^{(\mathrm{b})}$ & 1,05 & 1,02 \\
$\begin{array}{c}\text { Fluoroindato } \\
(P G I Z C a)\end{array}$ & 4,8 & 446 & $448^{(\mathrm{c})}$ & 0,99 & 0,97 \\
\hline
\end{tabular}

Tabela 3.3 - Tabela comparando o tempo de vida de baixa concentração $t_{0}$ (obtido com a Eq. (3.3)) e o tempo de vida radiativo, $t_{R}$, calculado pelo método de JO. ? ${ }_{\text {JO }}$ obtido pelo método de Judd-Ofelt e ? o obtido por LT. (a) Ref. [71]; (b) Ref. [72]; (c) Ref. [89]. 


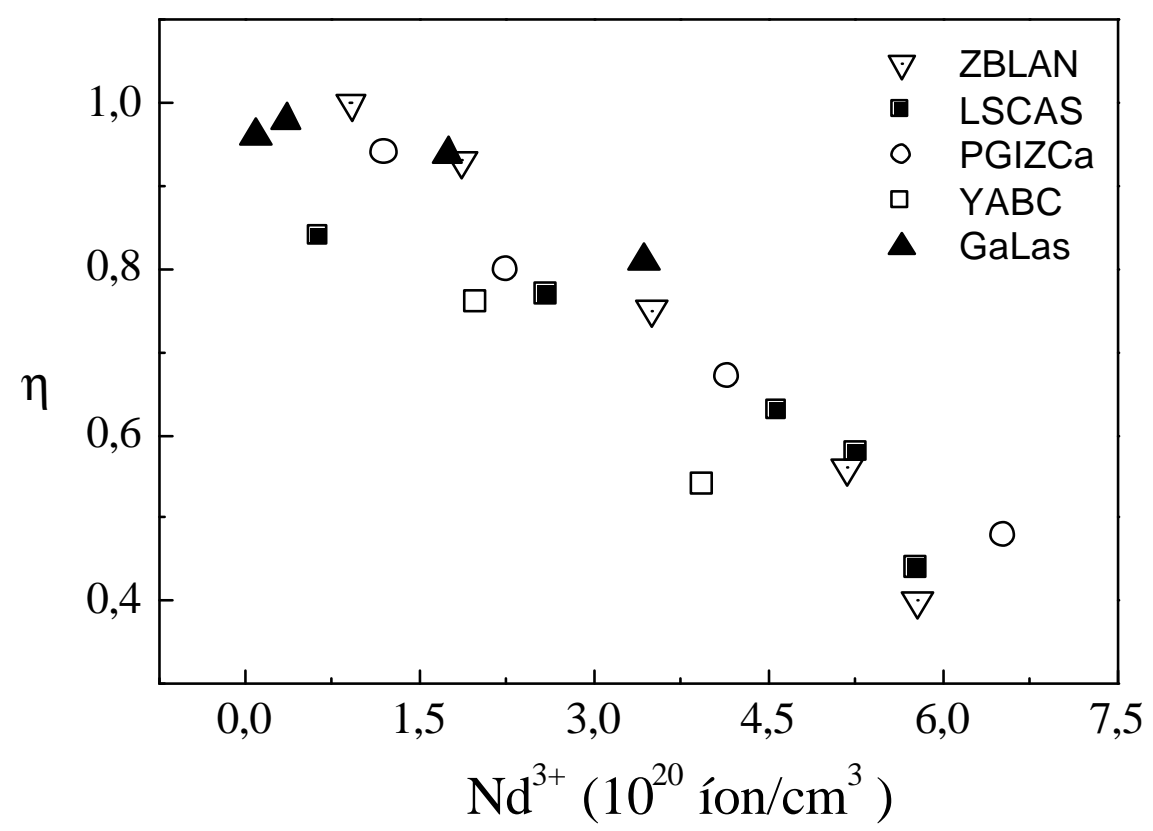

Figura 3.13 - Eficiência quântica, $\eta$, versus a concentração de $\mathrm{Nd}^{3+}$ para $Y A B C$, ZBLAN, PGIZCa LSCAS e GaLaS. 


\begin{tabular}{|c|c|c|c|c|c|c|}
\hline Amostras & $\begin{array}{c}\mathrm{A} \\
\left(\mathrm{cm}^{-1}\right) \\
\pm 4 \% \\
\end{array}$ & $\begin{array}{c}\mathrm{L} \\
(\mathrm{cm}) \\
\pm 2 \% \\
\end{array}$ & $\begin{array}{l}\mathrm{t}_{\exp } \\
(\mu s) \\
\pm 5 \%\end{array}$ & $\begin{array}{c}\mathrm{k}^{-1} \mathrm{ds} / \mathrm{dT}^{(\mathrm{a})} \\
\left(10^{-4} \mathrm{cmK}^{-1}\right) \\
\pm 5 \%\end{array}$ & $\begin{array}{c}\varphi_{\mathrm{Nd}} \\
\pm 10 \%\end{array}$ & $\begin{array}{r}? \\
+8 \%\end{array}$ \\
\hline $\begin{array}{c}\text { Aluminato } \\
\text { Base }\end{array}$ & 0,073 & 0,36 & - & 7,3 & 1 & - \\
\hline $0,5 \%$ & 0,84 & 0,168 & 320 & 7,3 & 0,35 & 0,84 \\
\hline $2,0 \%$ & 3,3 & 0,18 & 260 & 7,3 & 0,40 & 0,78 \\
\hline $3,5 \%$ & 5,44 & 0,10 & 190 & 7,3 & 0,52 & 0,63 \\
\hline $4,0 \%$ & 6,2 & 0,115 & 170 & 7,3 & 0,55 & 0,58 \\
\hline $4,5 \%$ & 6,94 & 0,078 & 153 & 7,3 & 0,66 & 0,44 \\
\hline $\begin{array}{c}\text { Fluoraluminato } \\
\qquad 1,0 \%\end{array}$ & 3,1 & 0,23 & 520 & $-2,4^{(a)}$ & 0,41 & 0,76 \\
\hline $2,0 \%$ & 6,9 & 0,22 & 430 & $-2,4$ & 0,58 & 0,54 \\
\hline $\begin{array}{c}\text { Fluoroindato } \\
0,56 \%\end{array}$ & 2,4 & 0,24 & 421 & $-2,3^{(a)}$ & 0,23 & 0,94 \\
\hline $1,0 \%$ & 4,1 & 0,24 & 367 & $-2,3$ & 0,38 & 0,80 \\
\hline $2,0 \%$ & 7,7 & 0,24 & 248 & $-2,3$ & 0,48 & 0,67 \\
\hline $3,0 \%$ & 12,6 & 0,22 & 164 & $-2,3$ & 0,62 & 0,48 \\
\hline $\begin{array}{c}\text { Fluorozirconato } \\
0,5 \%\end{array}$ & 1,77 & 0,23 & 515 & $-8,9^{(a)}$ & 0,21 & 1,00 \\
\hline $1,0 \%$ & 2,8 & 0,23 & 460 & $-8,9$ & 0,28 & 0,93 \\
\hline $2,0 \%$ & 5,25 & 0,24 & 380 & $-8,9$ & 0,41 & 0,75 \\
\hline $3,0 \%$ & 7,57 & 0,23 & 260 & $-8,9$ & 0,57 & 0,56 \\
\hline $3,5 \%$ & 9,1 & 0,26 & 230 & $-8,9$ & 0,69 & 0,40 \\
\hline
\end{tabular}

Tabela 3.4 - Parâmetros termo óptico experimental dos vidros: Aluminato (LSCAS) com os resultados em $\lambda_{\text {exc }}=810 \mathrm{~nm}$; Fluoraluminato (YABC), Fluoroindato (PGIZCa) $e$ Fluorozirconato (ZBLAN) com resultados obtidos em $\lambda_{\text {exc }}=797 \mathrm{~nm} . t_{\text {exp }}$ representa o valor experimental do tempo de vida. Nos fluoretos $\left\langle ?_{e m}^{-1}\right\rangle=0,97 \mu m^{-1}$ [72]. (a) Dados obtidos da Ref. [7]. 


\subsection{Medida de LT em função do comprimento de onda de excitação.}

Neste item, descreveremos um método que permite a determinação absoluta de ? sem a necessidade de utilização de amostra de referência. Neste caso mede-se o parâmetro $\mathrm{T}$ em função do comprimento de onda de excitação ? exc [32]. Os sólidos dopados com $\mathrm{Nd}^{3+}$ são particularmente favoráveis para esta técnica porque toda a fluorescência vem do nível ${ }^{4} \mathrm{~F}_{3 / 2}$, tal que $\left\langle ?_{\mathrm{em}}^{-1}\right\rangle$ não varia. Portanto, ambos ? e $\left\langle\lambda_{e m}^{-1}\right\rangle$ não dependem de $?_{\text {exc }}$, para excitações em ressonância com o nível do $\mathrm{Nd}^{3+}$. Consequentemente, pela Eq. (3.4), T $\propto \varphi \propto\left[1-?\left(?_{\text {exc }}\left\langle ?_{\text {em }}^{-1}\right\rangle\right)\right]$ e o ajuste de $\Theta$ por $?_{\text {exc }}$ pode ser usado para determinar $\eta$.

A Figura 3.14 mostra o resultado de $\mathrm{T}_{\mathrm{Nd}}$ para o LSCAS dopado com $2 \%$ de $\mathrm{Nd}^{3+}$. A partir do ajuste com a Eq. (3.4) nós obtivemos $\left(\mathrm{k} ?_{\mathrm{p}}\right)^{-1} \mathrm{ds} / \mathrm{dT}=(12,3 \pm 0,5) \mathrm{W}^{-1}$ e $\eta=0,82 \pm 0,03$. Este valor de $\left(\mathrm{k}{ }_{\mathrm{p}}\right)^{-1} \mathrm{ds} / \mathrm{dT}$ está em razoável acordo com o valor $11,5 \mathrm{~W}^{-1}$ para a amostra referência (não dopada). $\mathrm{O}$ valor de $?=0,82 \pm 0,03$ é comparável com $?=0,78 \pm 0,08$ obtido na seção anterior (3.2). 


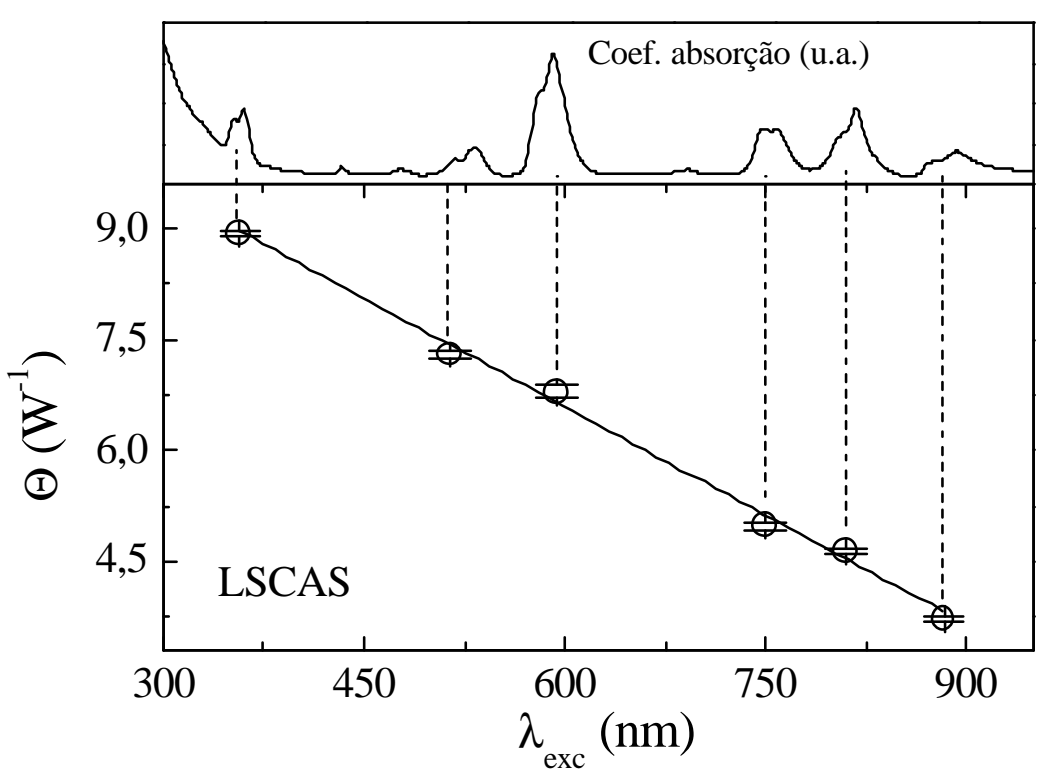

Figura 3.14 - Sinal de lente térmica normalizado versus o comprimento de onda de excitação no aluminato ( $L S C A S$ ) com 2,0\% $\mathrm{Nd}^{3+}$. Comprimento de onda de prova $?_{p}=632 \mathrm{~nm}$.

O mesmo procedimento foi utilizado no vidro ZBLAN dopado com $0,5 \%$ de $\mathrm{Nd}^{3+}$ (Figura 3.15) e no YAG dopado com $0,75 \%$ de $\mathrm{Nd}^{3+}$ (Figura 3.16). Os dados referentes a amostra de YAG encontram-se detalhados no Apêndice B Para o ZBLAN obtivemos $\left(\mathrm{k} ?_{\mathrm{p}}\right)^{-1} \mathrm{ds} / \mathrm{dT}=(13,7 \pm 0,9) \mathrm{W}^{-1}$ e $?=(0,99 \pm 0,03)$. Estes valores estão em bom acordo com $\left(\mathrm{k} ?_{\mathrm{p}}\right)^{-1} \mathrm{ds} / \mathrm{dT}=14,07 \mathrm{~W}^{-1}$ e $?=1,0$ apresentados na Tabela 3.4.

Para o YAG obtivemos $?=0,95 \pm 0,09$ e $\left(\mathrm{k}{ }_{\mathrm{p}}\right)^{-1} \mathrm{ds} / \mathrm{dT}=(1,67 \pm 0,02) \mathrm{W}^{-1}$. Nosso resultado de ? está em bom acordo com o valor $?=0,97 \pm 0,02$ obtido por Rosencwaig e Edward [66] (vide Figura 3.6). De fato, nosso valor de 0,95 é realmente um pouco maior do que $0,91-0,88$, valor que seria predito pelos cálculos de Judd-Ofelt e pelas medidas do tempo de vida da fluorescência. No entanto, nós acreditamos que esta pequena diferença nos resultados é conseqüência de erros 
cometidos nas medidas, que no nosso caso pode chegar a $\pm 10 \%$. Uma outra explicação para esta variação nos valores medidos, pode ser devido as diferentes concentrações de íons de $\mathrm{Nd}^{3+}$ nas amostras.

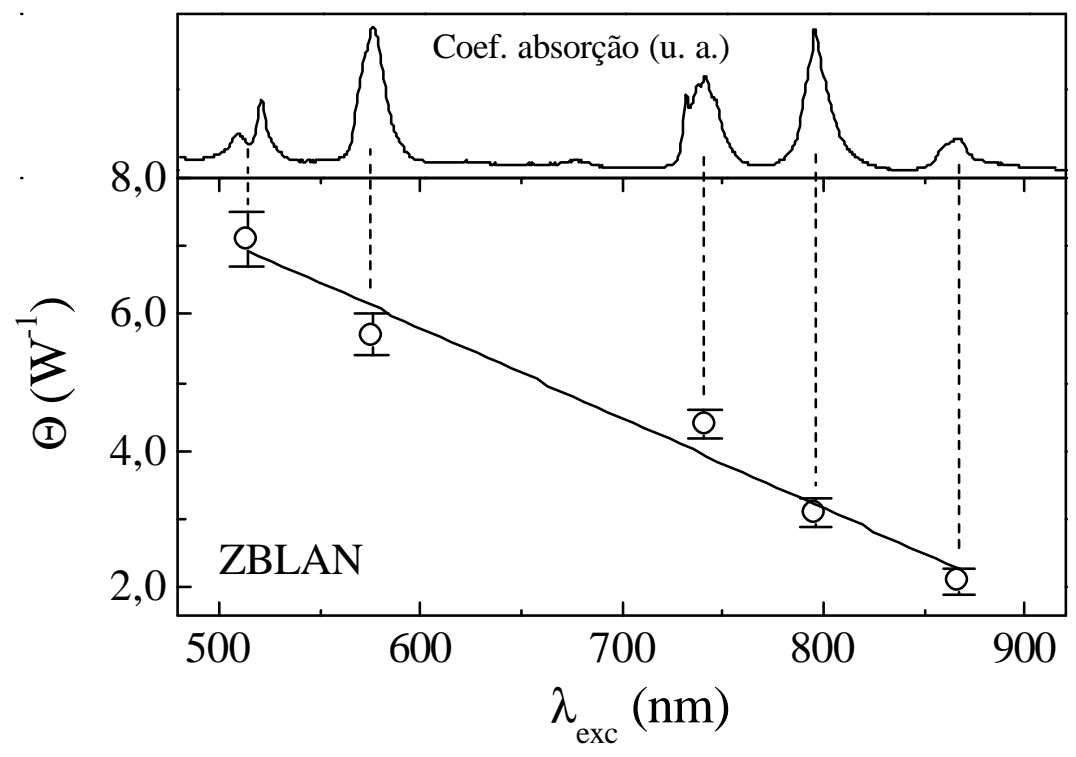

Figura 3.15 - Sinal de lente térmica normalizado versus o comprimento de onda de excitação, no fluorozirconato ( ZBLAN ) com 0,5\% $\mathrm{Nd}^{3+}$. Comprimento de onda de prova $?_{p}=632 \mathrm{~nm}$.

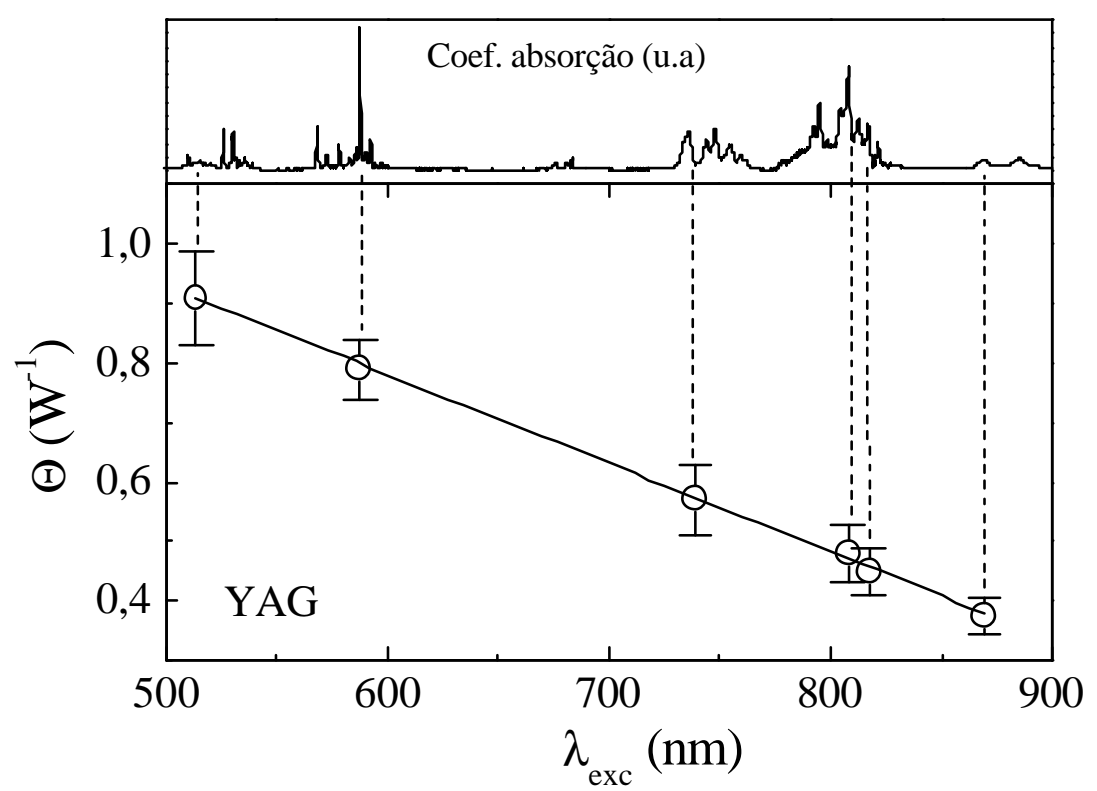

Figura 3.16 - Medidas de lente térmica em função do comprimento de onda no YAG. Espessura da amostra $L=2,1 \mathrm{~mm}$ e concentração de $0,75 \%$ de $\mathrm{Nd}^{3+}$. Comprimento de onda de prova $?_{p}=632 \mathrm{~nm}$ e $\left\langle ?_{e m}^{-1}\right\rangle=0,952 \mu \mathrm{m}^{-1}$ 


\subsubsection{Discussão.}

Para analisar a dependência da eficiência quântica com a concentração, podemos rescrever a Eq. (3.2) como $\mathrm{W}_{\mathrm{T}}=\mathrm{W}_{0}+\mathrm{W}_{\mathrm{rc}}$, onde $\mathrm{W}_{0}=\mathrm{W}_{\mathrm{R}}+\mathrm{W}_{\mathrm{mp}}+\mathrm{W}_{\mathrm{I}}$. Notamos ainda que $?^{-1}=\mathrm{W}_{\mathrm{T}} / \mathrm{W}_{\mathrm{R}}=\left(\mathrm{W}_{0}+\mathrm{W}_{\mathrm{rc}}\right) / \mathrm{W}_{\mathrm{R}}$ (Eq. (3.1)). Desta forma temos que:

$$
?^{-1}-?_{0}^{-1}=\frac{\mathrm{W}_{\mathrm{rc}}}{\mathrm{W}_{\mathrm{R}}}
$$

onde $?_{0}^{-1}=\mathrm{W}_{0} / \mathrm{W}_{\mathrm{R}}$. O termo $?^{-1}-?_{0}^{-1}$ representa apenas a contribuição da interação entre íons. Esta análise supõe que $\mathrm{W}_{0}$ não depende da concentração de $\mathrm{Nd}^{3+}$.

Convém ainda relacionar a Eq. (3.6) com a expressão empírica (Eq. (3.3)) que descreve a dependência do tempo de vida $\left(\mathrm{t}_{\exp }\right)$ com a concentração de íons. Lembrando que $?^{-1}=\mathrm{t}^{-1} \mathrm{t}_{\mathrm{R}}$, a Eq. (3.3) pode ser rescrita em termos da eficiência quântica:

$$
?^{-1}=?_{0}^{-1}\left[1+\left(\frac{\mathrm{N}_{0}}{\mathrm{~N}_{\mathrm{cq}}}\right)^{\mathrm{p}}\right]
$$

A Figura 3.17 mostra o gráfico de $?_{\mathrm{LT}}^{-1}$ (onde $?_{\mathrm{LT}}$ é a eficiência quântica obtida pelo método de LT) em função da concentração de íons de $\mathrm{Nd}^{3+}$ para os vidros LSCAS, ZBLAN e PGIZCa . As curvas foram ajustadas com a Eq. (3.7), de onde obtivemos o parâmetro $?_{0}^{-1}=1,18 ; 0,979 ; 1,03$, respectivamente. A Figura 3.18 mostra o gráfico (em escala logarítmica) de $\left(?_{0} / ?_{\mathrm{LT}}\right)-1$ (Eq. (3.7)) e $\left(\mathrm{t}_{0} / \mathrm{t}_{\text {exp }}\right)-1$ (Eq.(3.3)) em função da concentração de íons para as três amostras, respectivamente. 
Fazendo um ajuste linear dos dados experimentais, podemos determinar a concentração de "quenching" $\mathrm{N}_{\mathrm{cq}}$ (isto é, a concentração na qual a eficiência quântica e o tempo de vida caem pela metade) e o processo que determina a taxa de decaimento não radiativo. O valor da concentração de "quenching" corresponde ao valor da concentração na qual $\left(?_{0} / ?_{\mathrm{LT}}\right)-1=1$ e $\left(\mathrm{t}_{0} / \mathrm{t}_{\exp }\right)-1=1$. Os valores de $p$ obtidos dos ajustes do tempo de vida da fluorescência e da LT (vide Tabela 3.5) estão em bom acordo dentro do erro experimental.

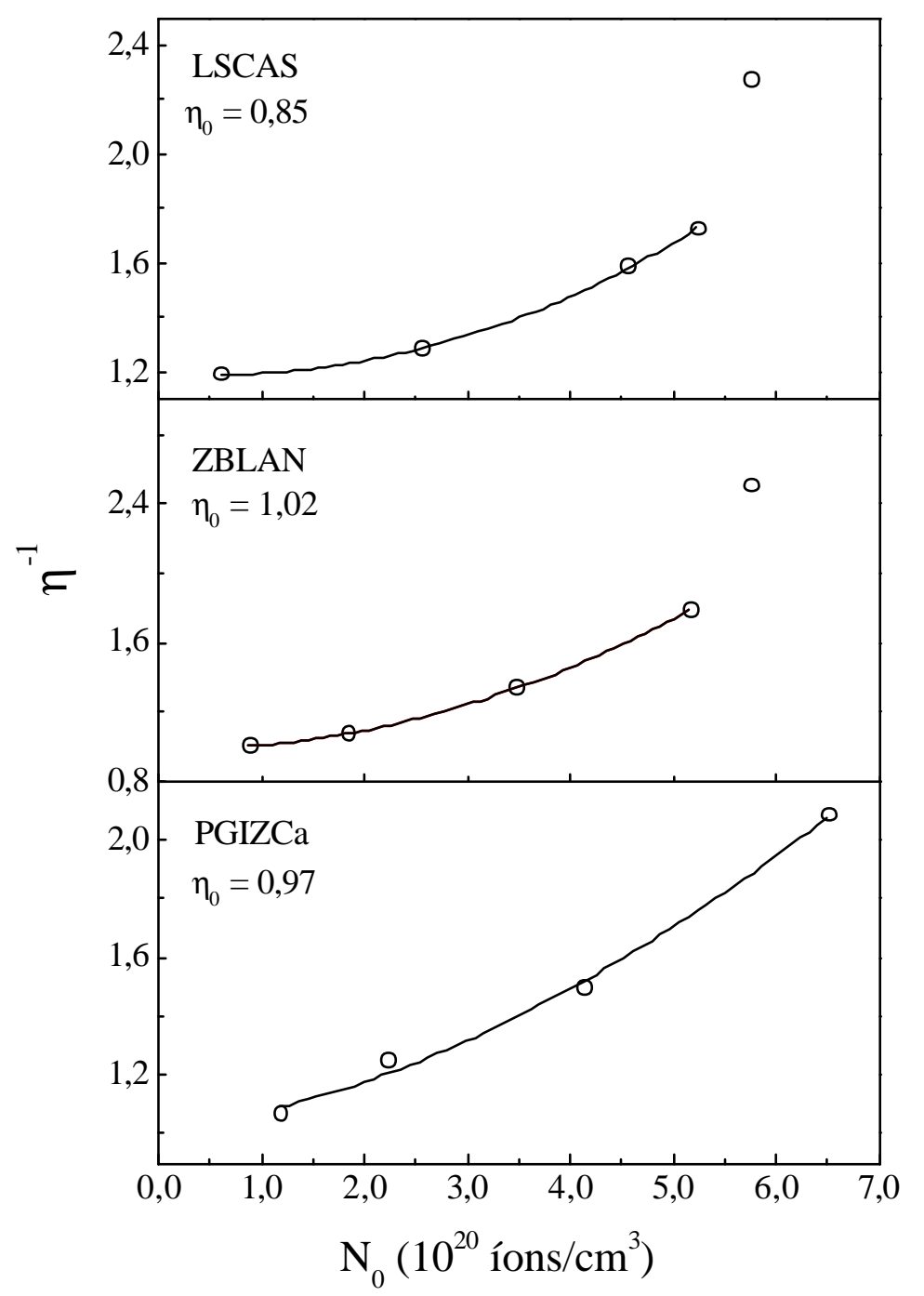

Figura 3.17 - Comportamento do inverso da eficiência quântica em função da concentração de $\mathrm{Nd}^{3+}$ para os vidros: LSCAS, ZBLAN e PGIZCa. Gráficos feitos com os dados da Tabela 3.4. A linha sólida representa o ajuste feito com a Eq. (3.7). 


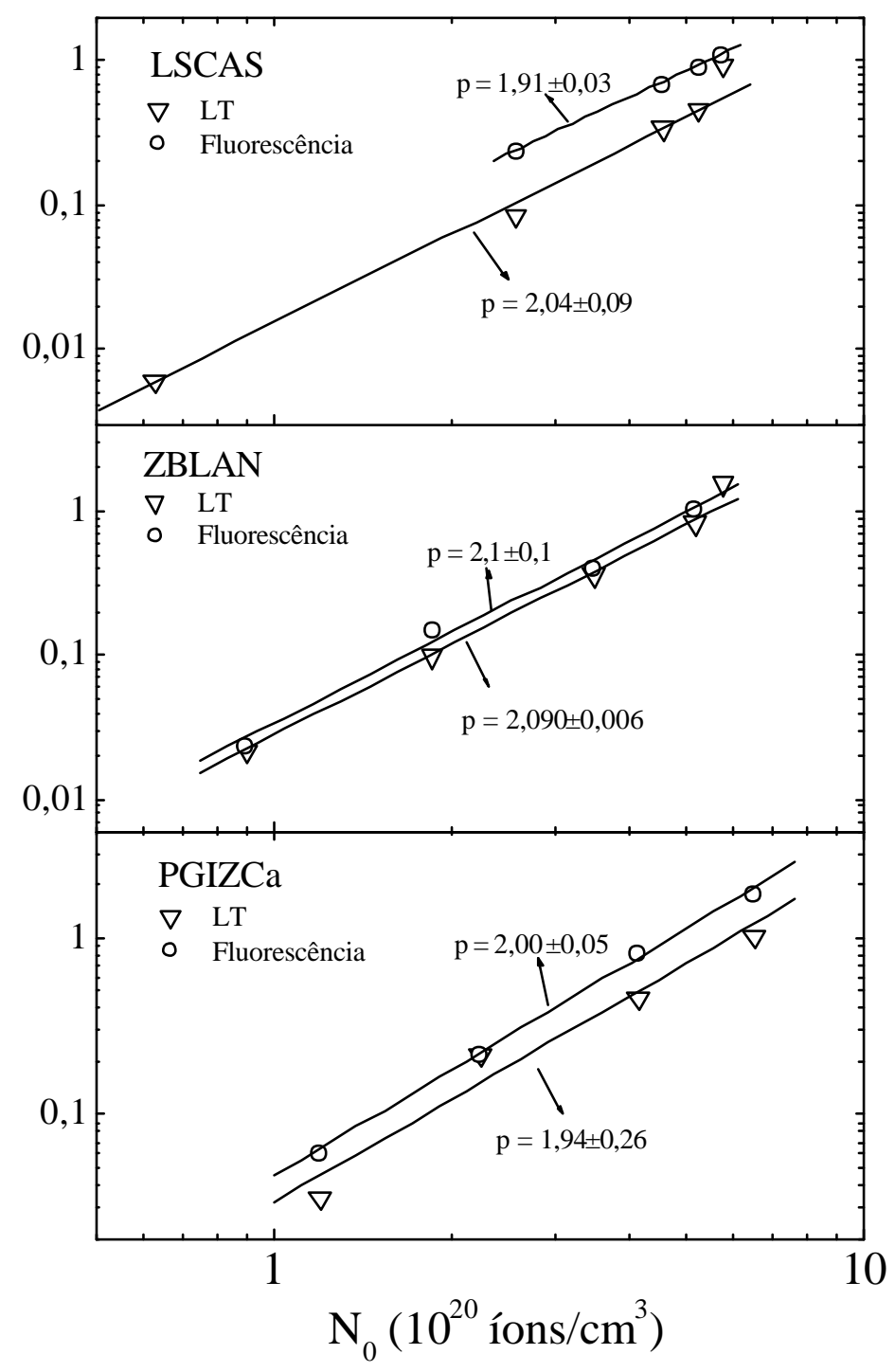

Figura 3.18 - O símbolo (o) representa o gráfico de $\left(t_{0} / t_{\text {exp }}\right)-1$ e $(\nabla)$ o gráfico de $\left(?_{0} / ?_{L T}\right)-1$, ambos em função da concentração de $\mathrm{Nd}^{3+}$. A linha sólida é o ajuste linear dos dados.

Para efeito de comparação com os resultados obtidos em nossas amostras, nós também analisamos os dados dos vidros calcogeneto, fluorozirconato (ZBLAN), fosfato (LG-750) e silicato (ED-2), cujos dados foram tirados da literatura. As respectivas referências encontram-se na legenda da Figura 3.19 e os resultados da análise podem ser vistos na Tabela 3.5. 
Os resultados do parâmetro $p$, obtidos pelos dois métodos Judd-Ofelt e LT, mostra que na maioria das amostras apresentadas na Tabela 3.5, a taxa de decaimento não radiativo pode ser atribuída a um processo de interação entre dois íons. A diferença entre os resultados de eficiência quântica, ? ${ }_{\text {JO }}$ e ? ${ }_{\text {LT }}$, pode ser entendida como sendo o erro cometido no cálculo do tempo de vida radiativo que varia entre $10-15 \%$ [46] e talvez por impurezas presentes nas amostras.
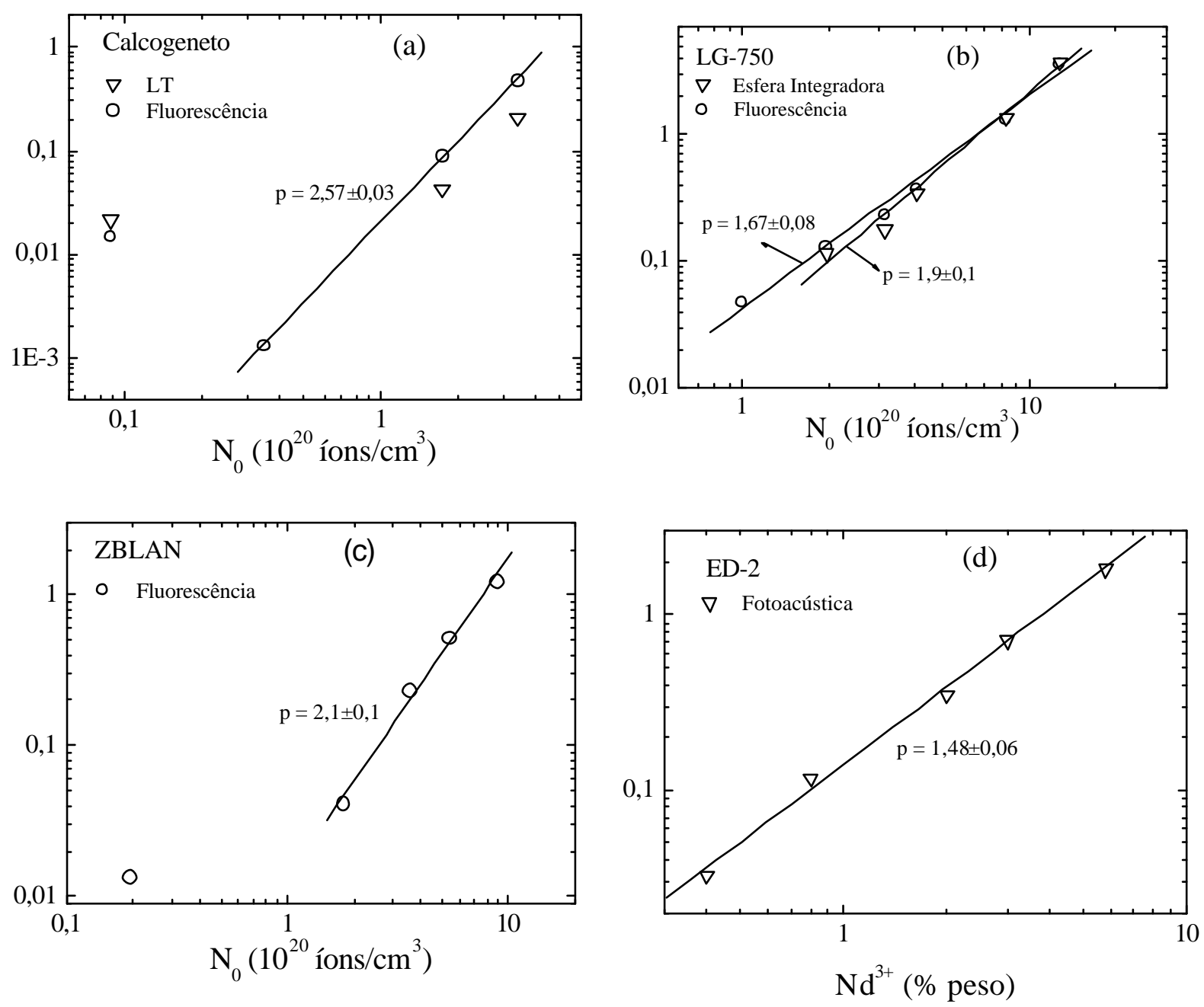

Figura 3.19 - O símbolo (o) representa o gráfico de $\left(t_{0} / t_{\text {exp }}\right)-1$ e $(\nabla)$ o gráfico de $\left(?_{0} /\right.$ ?) - 1. Os dados de (a) foram tirados da Ref. [43]; (b) da Ref. [85]; (c) da Ref. [72] e (d) da Ref. [66]. 


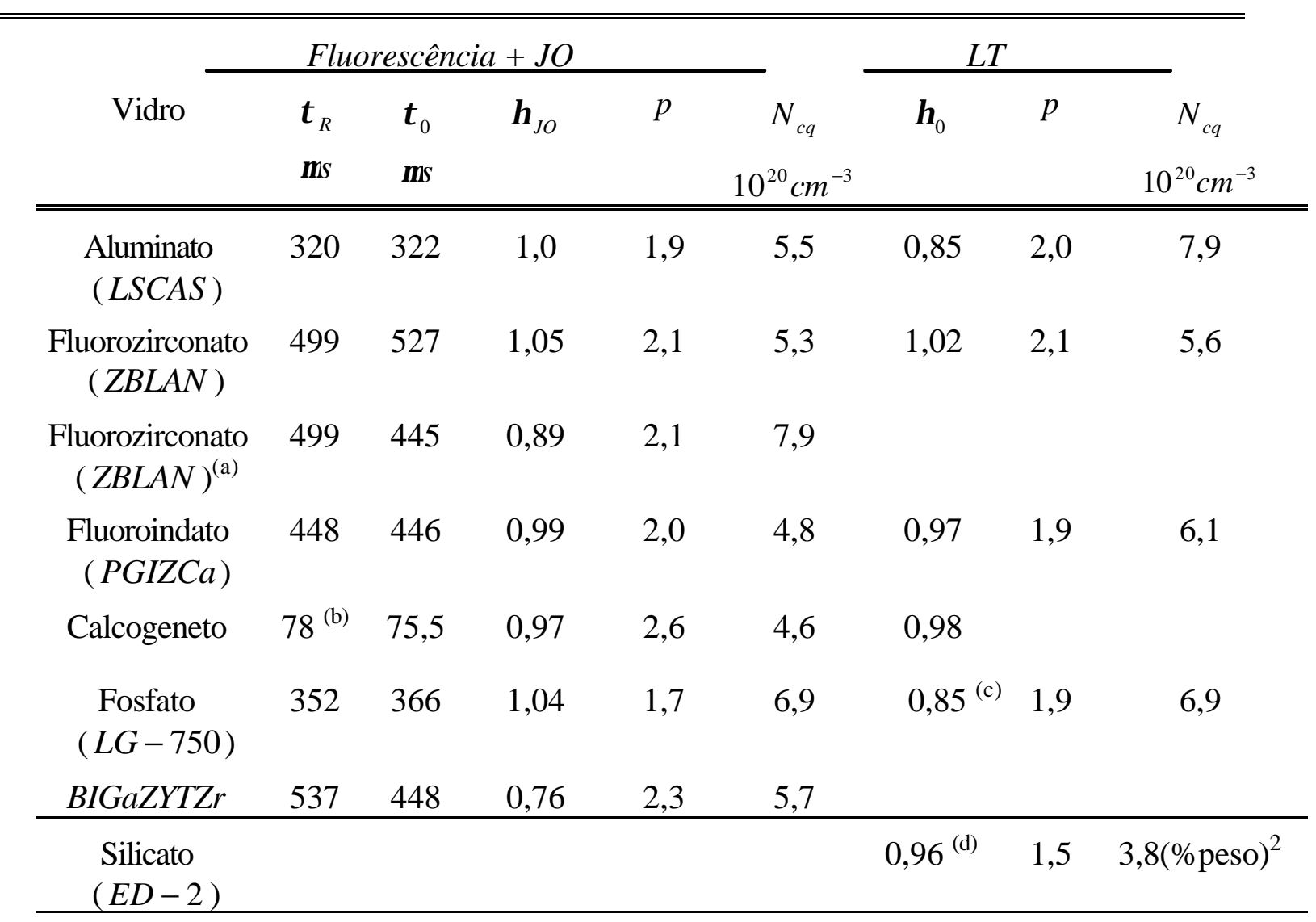

Tabela 3.5 - Tabela com os parâmetros obtidos através das medidas de LT e do tempo de vida. (a) Dados tirados da Ref. [72], (b) dado tirado da Ref. [30]; (c) obtido por esfera integradora [85]; (d) obtido por fotoacústica [66].

\subsection{Medidas de LT no filme de PPV (p-fenileno-vinileno).}

Os vidros e cristais dopados com $\mathrm{Nd}^{3+}$ têm sido muito estudados nas últimas três décadas. Agora, pretendemos mostrar que a aplicação do método de LT também pode ser aplicado a filmes poliméricos.

No item anterior mostramos que, quando toda fluorescência vem de um mesmo nível, como é o caso dos sólidos dopados particularmente com $\mathrm{Nd}^{3+}$ tal que $\left\langle ?_{\mathrm{em}}^{-1}\right\rangle$ não varia, o método de LT variando o comprimento de onda de excitação pode ser aplicado para determinar a eficiência quântica ?. Neste item, mostraremos que o

\footnotetext{
${ }^{2}$ Para esta amostra não encontramos o valor da concentração em íons $/ \mathrm{cm}^{3}$.
} 
filme de PPV (p-fenileno-vinileno), também é possível de ser estudado utilizando este método de LT. Isto é possível, como veremos a seguir, porque como nos sólidos dopados, o PPV também apresenta um intervalo cuja variação do comprimento de onda de excitação não altera o valor da eficiência quântica, de modo que a técnica de LT variando o comprimento de onda pode ser aplicada.

Inicialmente vamos analisar algumas características dos filmes de PPV (pfenileno-vinileno). A Figura 3.20 mostra espectros típicos de absorbância e luminescência de um filme PPV tratado a $110^{\circ} \mathrm{C}$.

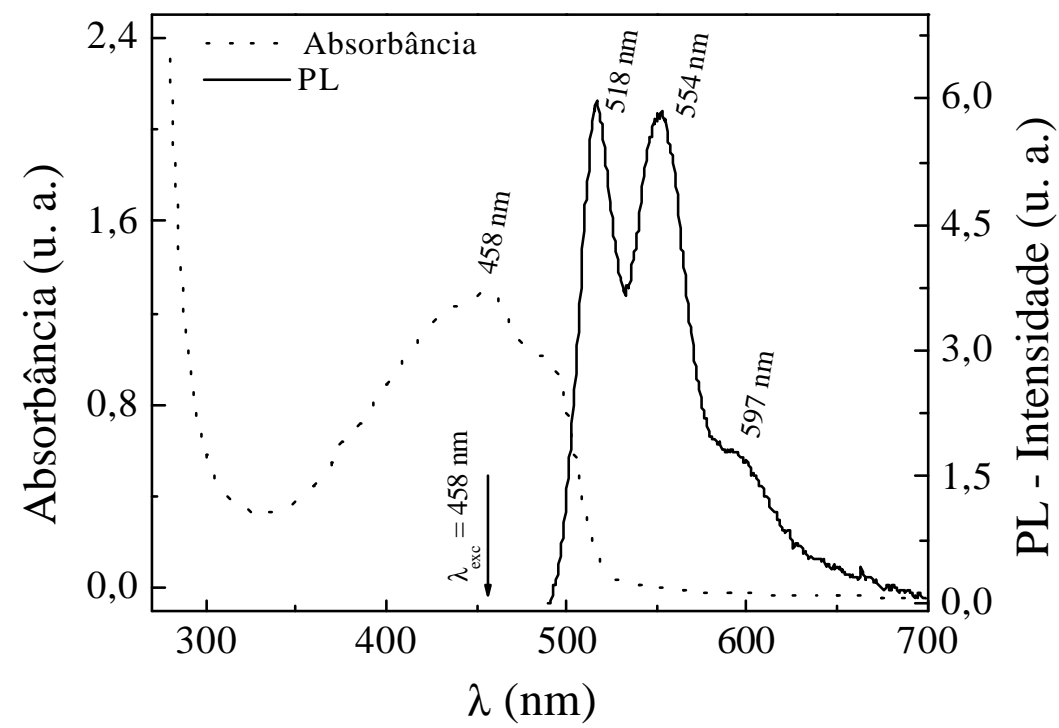

Figura 3.20 - Espectro de absorção ótica e fotoluminescência $\left(T=300^{\circ} \mathrm{K}\right)$ do filme PPV tratado a $110^{\circ} \mathrm{C}$. As medidas foram realizadas pelo Dr. A. Marlleta no grupo de polímeros do IFSC.

A medida de absorbância, Figura 3.20, no UV-Vis foi realizada em um espectrofotômetro Hitachi U-2001. O espectro é composto por uma banda larga centrada em $458 \mathrm{~nm}$. No caso da fotoluminescência, denominado PL (do inglês "photoluminescence"), o espectro foi obtido com a seguinte montagem experimental: um laser de $\mathrm{Ar}^{+}$excitando a amostra em 458nm, a emissão analisada por um monocromador DIGIKROM DK480 e detectada por uma fotomultiplicadora tipo 
Hamamatsu R446. A amostra foi condicionada em um criostato de circuito de hélio, fechado sob vácuo de $10^{-5}$ torr. O espectro apresenta três picos centrados em 518 , 554 e $597 \mathrm{~nm}$.

$\mathrm{Na}$ Figura 3.21, temos o espectro de excitação da fotoluminescência, usualmente denominado PLE, do inglês "photoluminescence excitation". O espectro foi obtido pelo monitoramento da intensidade da fotoluminescência (PL) em um comprimento de onda de detecção fixo em 500nm (vide Figura 3.20) e variando o comprimento de onda de excitação. As medidas foram realizada em um espctrômetro de luminescência Perkin Elmer LS50B no laboratório do Prof. Luis Antônio. Normalizando o sinal da PLE pela absorbância e pelo comprimento de onda de excitação podemos observar que no intervalo de $450-500 \mathrm{~nm}$ a eficiência quântica é aproximadamente constante, como pode ser visto na Figura 3.22. Os pontos da Figura 3.22 representam a intensidade da luminescência obtida durante o experimento de LT (excitando com o laser de $\mathrm{Ar}^{+}$). Como no caso da linha contínua, os pontos foram normalizados pela absorbância e pelo comprimento de onda de excitação.

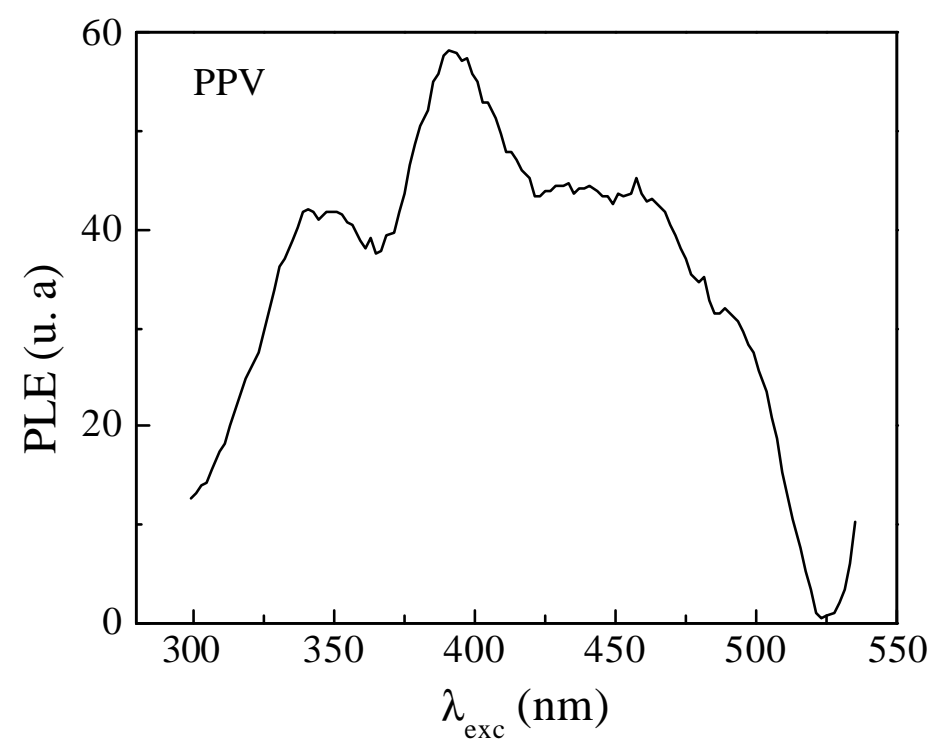

Figura 3.21 - Espectro de excitação da fotoluminescência (PLE). 


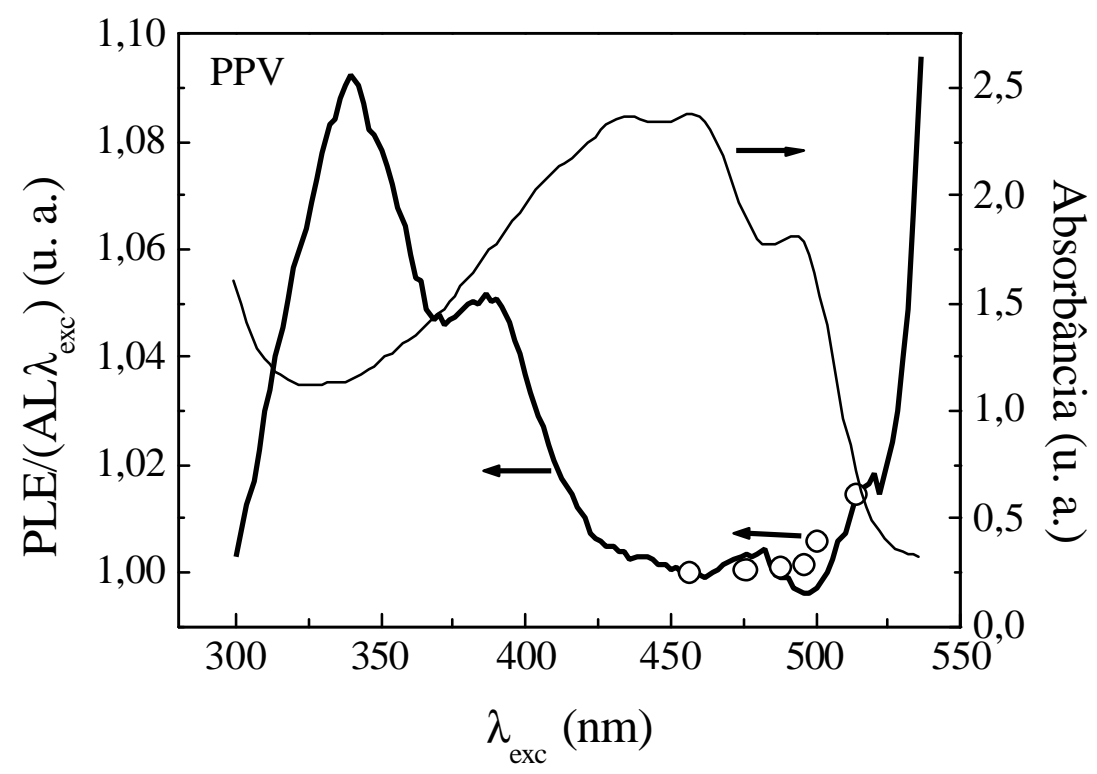

Figura 3.22 - Sinal da fotolumenescência (linha forte) e intensidade da luminescência (o). Ambos os sinais foram normalizados pela absorbância (linha fraca) e pelo comprimento de onda de excitação. Os resultados estão apresentados mesma escala.

Uma vez determinado o intervalo de comprimento de onda, no qual a eficiência quântica permanece constante, as medidas de LT foram realizadas. E para comprovar que eficiência quântica assume diferentes valores fora da região determinada, nós excitamos a amostra em 7 diferentes comprimentos de onda. A Figura 3.23 mostra que, no intervalo de 450 a $500 \mathrm{~nm}$, o parâmetro $\mathrm{T}=$ ? $/ \mathrm{PAL}_{\text {eff }}$ decresce linearmente com $?_{\text {exc }}$, o que comprova que neste intervalo a eficiência quântica é constante, como observado na Figura 3.22. Fazendo o ajuste com a Eq. (3.4) para os quatro pontos compreendidos no intervalo de 450 a 500nm, nós obtivemos $\left(\mathrm{k} ?_{\mathrm{p}}\right)^{-1} \mathrm{ds} / \mathrm{dT}=(42,9 \pm 2,3) \mathrm{W}^{-1}$ e $?=(0,85 \pm 0,15)$. Para os pontos que se encontram fora da curva (Figura 3.23), calculamos uma eficiência quântica relativa. $\mathrm{O}$ cálculo foi feito tomando o valor de $\mathrm{T}$ em cada ponto e assumindo como constante o valor de $\left(\mathrm{k}{ }_{\mathrm{p}}\right)^{-1} \mathrm{ds} / \mathrm{dT}$. Os resultados estão apresentados na Tabela 3.6. 


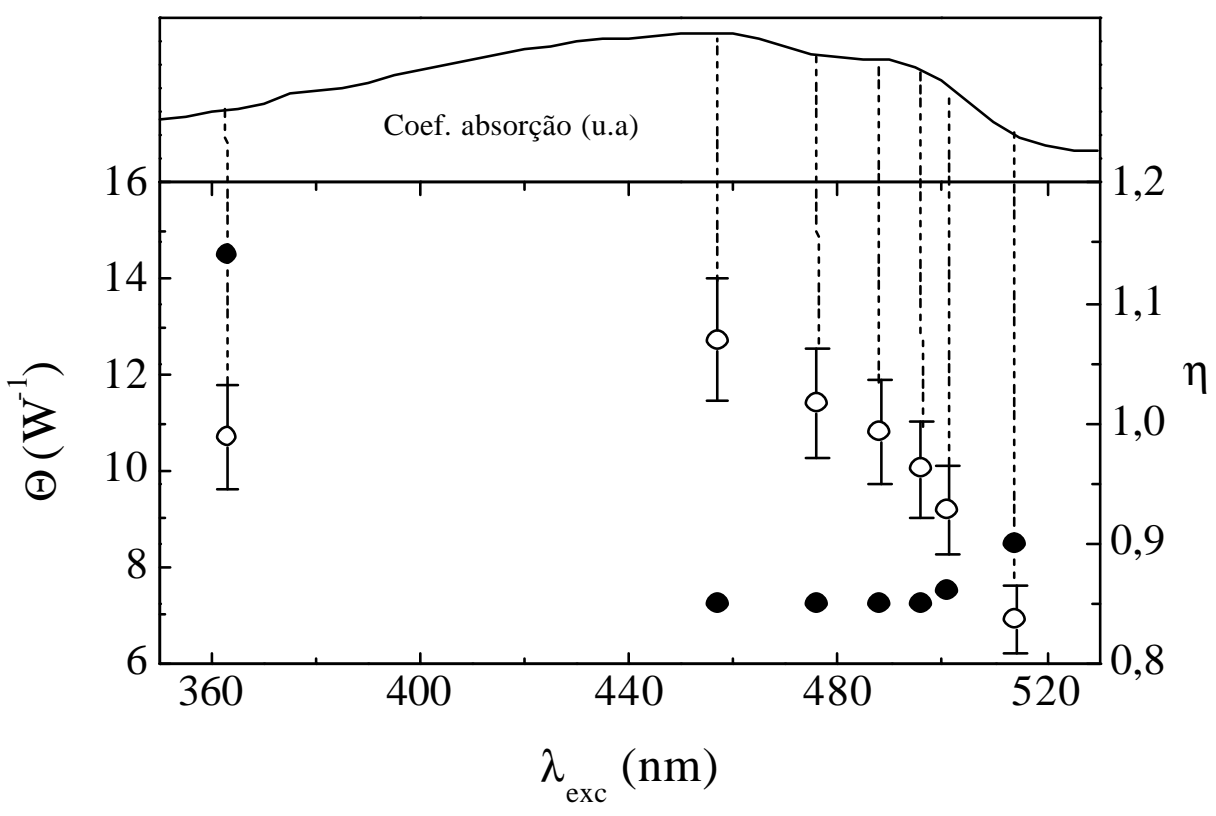

Figura 3.23 - Sinal de lente térmica normalizado versus o comprimento de onda de excitação para o PPV. Comprimento de onda de prova $?_{p}=632 \mathrm{~nm} e$ $\left\langle ?_{\text {em }}^{-1}\right\rangle=1,81 \mu \mathrm{m}^{-1}$.

\begin{tabular}{cc}
\hline \hline$\lambda(\mathrm{nm})$ & $\eta ? \pm 15 \%)$ \\
\hline \hline $450-500$ & 0,85 \\
363 & 1,14 \\
501 & 0,86 \\
514 & 0,90 \\
\hline
\end{tabular}

Tabela 3.6 - Eficiência quântica do PPV determinada pelo método de lente térmica.

Da literatura é esperado que a eficiência quântica, ?, seja maior do que $50 \%$ em filmes de PPV parcialmente conjugados [90, 91]. Nossos resultados indicam que o filme de PPV termicamente convertido a baixa temperatura apresenta poucos defeitos estruturais (relacionados com canais não radiativos, como grupos carbonila). 


\section{Capítulo 4}

\section{Medidas de $d s / d T$.}

Neste capítulo iremos apresentar os resultados do parâmetro ds/dT. Os resultados mostram a potencialidade do método de LT para obter o valor absoluto deste importante parâmetro ótico, o qual é crucial para quem trabalha no projeto de cavidade de laseres de estado sólido. Faremos também uma comparação entre os valores de ds/dT obtidos experimentalmente com a técnica de LT com os valores calculados através da Eq. (4.5). Aqui é importante lembrar que, para o obter o parâmetro ds/dT por meio de medidas de LT, é preciso conhecer não só a condutividade térmica do material em questão, mas também a sua eficiência quântica, ? , a qual já foi apresentada no Capítulo 3.

O parâmetro ds/dT, o qual descreve a distorção induzida termicamente por um feixe de laser durante sua passagem em um meio absorvedor, é uma das propriedades mais importantes de materiais óticos. Este parâmetro carrega informações sobre o coeficiente de expansão térmica da amostra, índice de refração e coeficiente de tensão ótica. É bem conhecido que a combinação destas propriedades podem ser usadas para minimizar o valor de ds/dT. Isto é feito no projeto de materiais óticos atérmico, isto é, materiais que apresentam $\mathrm{ds} / \mathrm{dT} \approx 0$, o que é muito importante para sistemas óticos que experimentam uma grande variação de temperatura, tal como bastão laser para sistemas de alta potência [16, 24, 32, 52]. Apesar de que vários métodos terem sido desenvolvidos para investigar ds/dT, uma determinação precisa de seu valor absoluto tem se mostrado muito difícil. Nas 
medidas de LT, ds/dT é um dos parâmetros que pode ser medido quantitativamente $[6,24]$.

Originalmente, a técnica de LT e sua teoria foram desenvolvidas principalmente considerando experimentos em amostras líquidas, onde $\mathrm{ds} / \mathrm{dT}=\mathrm{dn} / \mathrm{dT}$. No caso de amostras sólidas homogêneas, a teoria original pode ser aplicada simplesmente substituindo $\mathrm{dn} / \mathrm{dT}$ por ds/dT [6]. Para amostras homogêneas, o caminho ótico $\mathrm{S}(\mathrm{cm})$, é uma função da temperatura como $\mathrm{S}(\mathrm{T})=\mathrm{n}(\mathrm{T}) \mathrm{L}(\mathrm{T})$. O perfil de temperatura induzido pelo feixe de excitação radial, causa uma variação no caminho ótico [24]:

$$
? \mathrm{~S}=\left(\mathrm{n}_{0}-1\right) ? l+l_{0} ? \mathrm{n}
$$

onde ? l(r) está esquematizado na Figura 4.1. É importante lembrar que, ambos os fatores ? $l$ e $?$ n dependem da temperatura e da tensão (ou "stress") térmica [92]:

$$
\frac{? l}{l_{0}}=\mathrm{a} ? \mathrm{~T}+\frac{\mathrm{s}_{\mathrm{z}}}{\mathrm{Y}}-\frac{?}{\mathrm{Y}}\left(\mathrm{s}_{\mathrm{r}}+\mathrm{s}_{\mathrm{f}}\right)
$$

onde ? T é o aumento da temperatura induzida pelo laser, a $\left(\mathrm{K}^{-1}\right)$ o coeficiente de expansão térmica, Y o módulo de Young, ? a razão de Poisson, $\mathrm{s}_{\mathrm{r}}, \mathrm{s}_{\mathrm{z}}$ e $\mathrm{s}_{\mathrm{f}}$ são o tensor radial de tensão, as componentes axial e azimutal, respectivamente.

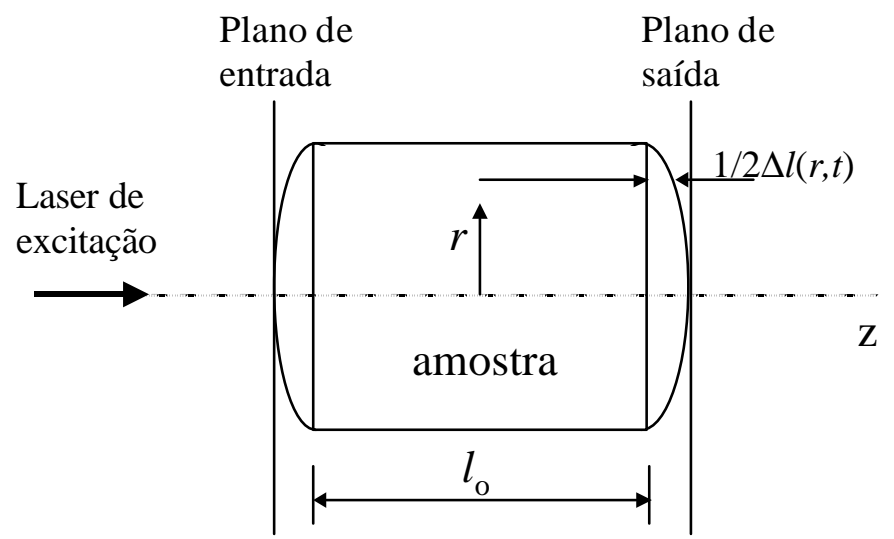

Figura 4.1 - Diagrama esquemático da variação do caminho ?l(r) induzida pelo laser. 
Com respeito a variação do índice de refração, nós consideramos $\left.? \mathrm{n}=? \mathrm{n})_{\text {tensão }}+\partial \mathrm{n} / \partial \mathrm{T}\right)_{\mathrm{s}=0} ? \mathrm{~T}$, onde $\left.\partial \mathrm{n} / \partial \mathrm{T}\right)_{\mathrm{s}=0}$ é o coeficiente termo ótico, $\mathrm{dn} / \mathrm{dT}$. ? n $)_{\text {tensão }}$ depende da direção de polarização $r$ ou $\phi$ [51].

No experimento de LT, o aquecimento da amostra pela radiação laser, induz uma variação de temperatura entre o centro e a borda, impedindo a expansão da parte central mais quente gerando a tensão (ou "stress"). No caso de geometria de disco fino (amostra com diâmetro $\mathrm{d}>>l_{\mathrm{o}}$ ) pode ser mostrado que [92]

$$
\begin{aligned}
& \frac{? l}{l}=\mathrm{a}(1+?) ? \mathrm{~T} \\
& ? \mathrm{n}=\left[\frac{\mathrm{dn}}{\mathrm{dT}}+\frac{\mathrm{n}_{0}^{3}}{4} \mathrm{Y}\left(\mathrm{q}_{/ /}+\mathrm{q}_{\perp}\right)\right] ? \mathrm{~T}
\end{aligned}
$$

onde $\mathrm{q}_{/ /}$e $\mathrm{q}_{\perp}$ são os coeficientes de tensão óptico para as orientações paralela $\mathrm{e}$ perpendicular relativa polarização do feixe de excitação. Substituindo (4.3) e (4.4) em (4.1) e chamando ds/dT $=l_{0}^{-1} \mathrm{dS} / \mathrm{dT}$, nós temos:

$$
\left.\frac{\mathrm{ds}}{\mathrm{dT}}=\frac{\mathrm{dn}}{\mathrm{dT}}+\mathrm{a}\left(\mathrm{n}_{0}-1\right)(1+?)+\frac{\mathrm{n}_{0}^{3}}{4} \mathrm{Ya}_{\left(\mathrm{q}_{/ /}\right.}+\mathrm{q}_{\perp}\right)
$$

Para facilitar a interpretação, vamos escrever a Eq. (4.5) como a soma de três termos:

$$
\frac{\mathrm{ds}}{\mathrm{dT}}=\frac{\mathrm{dn}}{\mathrm{dT}}+?_{\mathrm{a}}+?_{\mathrm{q}}
$$

Como mencionado no Capítulo 2, na medida de LT a área iluminada da amostra é aquecida e tende a se expandir. Como conseqüência das bordas estarem mais frias, a amostra impede a expansão gerando uma tensão ("stress"). Este efeito é calculado pelo terceiro termo da Eq. (4.6). Em líquidos, dn/dT geralmente é negativo porque o coeficiente de expansão térmica é o termo dominante na Eq. (4.6). 
Para muitos vidros óxidos e cristais, dependendo da estrutura, os três termos na Eq. (4.6) são positivos. Por outro lado, materiais com dn/dT negativo são desejáveis para minimizar ds/dT, e consequentemente, o efeito de lente térmica na ação laser.

Embora a expressão acima seja comumente usada, não temos conhecimento de nenhum experimento de LT em que ela tenha sido verificada experimentalmente.

\subsection{Resultados.}

Nós calculamos o parâmetro ds/dT para o YAG, tomando os dados experimentais obtidos no Capítulo 2 (vide Figura 2.10), onde do ajuste com a Eq. (2.3) foi obtido $?=(2,400 \pm 0,005) \times 10^{-2} \mathrm{rad}$. A Eq. (2.5) pode ser usada para obter ds/dT a partir de $\theta$ se $\varphi$ e outros parâmetros forem previamente determinados. Para o YAG a eficiência quântica é sabida para ser próximo da unidade (? 1), tal que usando $\lambda_{\text {exc }}=808 \mathrm{~nm}$ e $\left\langle ?_{\mathrm{em}}^{-1}\right\rangle=0,94 \mu \mathrm{m}^{-1}$ temos que $\varphi=0,24$. Portanto substituindo os valores de $\theta, \varphi$ e $\mathrm{k}=0,13 \mathrm{~W} / \mathrm{Kcm}$ na Eq. (2.5), obtivemos $\mathrm{ds} / \mathrm{dT})_{\mathrm{LT}}=14 \times 10^{-6} \mathrm{~K}^{-1}$. Nós comparamos este valor com o calculado pela Eq. (4.6). Usando os dados obtidos na literatura, calculamos $\mathrm{ds} / \mathrm{dT})_{\text {cal }}=(7,3+7,7+0,67) \times 10^{-6} \mathrm{~K}^{-1}=15,7 \times 10^{-6} \mathrm{~K}^{-1}$, onde os três termos estão escritos na mesma ordem como na Eq. (4.6). Portanto, para o YAG as contribuições dos termos dn/dT, ? ${ }_{a}$ e ? ${ }_{q}$ são $46 \%, 49 \%$ e $4 \%$, respectivamente. O ds/dT) $)_{\text {cal }}$ está em bom acordo com o obtido experimentalmente ds/dT) $)_{\mathrm{LT}}$. A discrepância de $\sim 10 \%$ é menor do que a soma de todas as incertezas experimentais envolvidas no cálculo de ds/dT) $)_{\text {cal }}$ e ds/dT) $)_{\mathrm{LT}}$. O mesmo processo foi usado para obter o valor de 
ds/dT) $)_{\text {LT }}$ para o LSCAS e o ZBLAN. Os resultados estão apresentados na Tabela 4.1.

\begin{tabular}{ccccccc}
\hline \hline Amostras & $\begin{array}{c}\mathrm{dn} / \mathrm{dT} \\
\left(10^{-6} K^{-1}\right)\end{array}$ & $\begin{array}{c}\mathrm{a} ? \\
\left(10^{-6} K^{-1}\right)\end{array}$ & $\begin{array}{c}\Delta_{\alpha} ? \\
\left(10^{-6} K^{-1}\right)\end{array}$ & $\begin{array}{c}\Delta_{q} \\
\left(10^{-6} K^{-1}\right)\end{array}$ & $\begin{array}{c}\mathrm{ds} / \mathrm{dT})_{\mathrm{cal}} \\
\left(10^{-6} K^{-1}\right)\end{array}$ & $\begin{array}{c}\mathrm{ds} / \mathrm{dT})_{\mathrm{LT}} \\
\left(10^{-6} K^{-1}\right)\end{array}$ \\
\hline \hline $\begin{array}{c}\text { YAG } \\
7,3^{(\mathrm{a})}\end{array}$ & $7,5^{(\mathrm{a})}$ & 7,7 & 0,67 & 15,7 & 14 \\
$\begin{array}{c}\text { Fluorozirconato } \\
(\text { ZBLAN })\end{array}$ & $-12^{\text {(b) }}$ & $14^{\text {(b) }}$ & 9,5 & 2,5 & 0,0 & $-6,3$ \\
$\begin{array}{c}\text { Aluminato } \\
(\text { LSCAS })\end{array}$ & $8,05^{\text {(c) }}$ & $8,2^{\text {(c) }}$ & 8,47 & 1,0 & 17,6 & 12 \\
\hline
\end{tabular}

Tabela 4.1 - Propriedade das amostras estudados. (b) Ref. [93]; (c) Ref. [94]; (a) Ref. [51]. $?_{a}=(n-1)(1+\mu) a$ e $?_{q}=n^{3} Y \alpha\left(q_{\perp}+q_{/ /}\right) / 4$.

$\mathrm{O}$ resultado obtido para o ZBLAN ds $/ \mathrm{dT})_{\mathrm{LT}}=-6,3 \times 10^{-6} \mathrm{~K}^{-1}$ discorda com $\mathrm{ds} / \mathrm{dT})_{\text {cal }}=(-12+9,5+2,5) \times 10^{-6} \mathrm{~K}^{-1} \sim 0 . \quad$ Existe muitas razões para esta discrepância. O $\mid \mathrm{ds} / \mathrm{dT})_{\text {cal }} \mid$ desprezível resulta de um cancelamento de contribuições positivas e negativas, como mencionadas no item anterior. Por outro lado, as incertezas experimentais de todas as contribuições sempre se somam, tal que estimando uma incerteza de $20 \%$ para cada termo, nós obtemos um erro em ds $/ \mathrm{dT})_{\text {cal }}$ da ordem de $\pm 5,0 \times 10^{-6} \mathrm{~K}^{-1}, \quad$ o qual se aproxima do valor $\mathrm{ds} / \mathrm{dT})_{\mathrm{LT}}=-(6,3 \pm 0,5) \times 10^{-6} \mathrm{~K}^{-1}$.

ZBLAN é um nome geral para vidros com muitos componentes fluorozirconatos e suas propriedades físicas variam muito com a composição [93]. Por exemplo, Izumitami e colaboradores mediram $\mathrm{dn} / \mathrm{dT}=-14,75 \times 10^{-6} \mathrm{~K}^{-1}$, $\mathrm{a}=17,2 \times 10^{-6} \mathrm{~K}^{-1}$ e $\mathrm{T}_{\mathrm{g}}=257^{\circ} \mathrm{C}$ [95]. Nossa amostra de ZBLAN apresenta relativamente um alto valor de $\mathrm{T}_{\mathrm{g}}\left(298^{\circ} \mathrm{C}\right)$, baixo coeficiente de expansão 
$\left(\mathrm{a}=14 \times 10^{-6} \mathrm{~K}^{-1}\right)$ e $\mathrm{dn} / \mathrm{dT}=-12 \times 10^{-6} \mathrm{~K}^{-1}$. A magnitude do termo ? ${ }_{\mathrm{q}}$ não foi calculada para a nossa especifica composição, o valor foi obtido do trabalho de Jewell e colaboradores [93], tal que a incerteza em $\Delta_{q}$ pode justificar a discrepância entre ds/dT) $)_{\text {cal }}$ e ds/dT) $)_{\mathrm{LT}}$. Também é interessante dizer que, para quatro diferentes composições de vidros fluoroindatos, com $\mathrm{T}_{\mathrm{g}} \sim 270-290^{\circ} \mathrm{C}$, apresenta ds/dT entre $-1,3$ e $-2,4 \times 10^{-6} \mathrm{~K}^{-1}$ [92], próximo ao valor calculado para o ZBLAN .

Nos cristais uniaxiais $\left(\mathrm{YVO}_{4}, \mathrm{YLF}, \mathrm{LiSAF}\right.$ e LiSGaF ) os parâmetros $\alpha, \mathrm{k}$ e $D$ dependem da orientação [63-65], as medidas de LT foram realizadas com o feixe de prova polarizado com o objetivo de verificar o comportamento da birrefringência de ds/dT. As respectivas orientações estão apresentadas na Figura 4.2. É importante lembrar que para o LiSAF e LiSGaF, $\alpha_{\perp c}$ é positivo e $\alpha_{/ / c}$ é negativo, conduzindo a uma grande tensão ("stress").

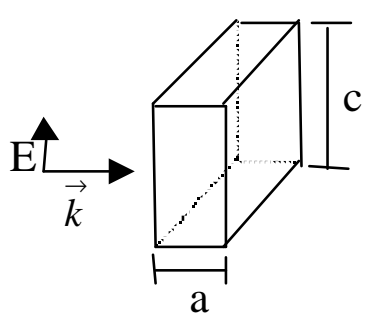

$\mathrm{E} / / \mathrm{c}$

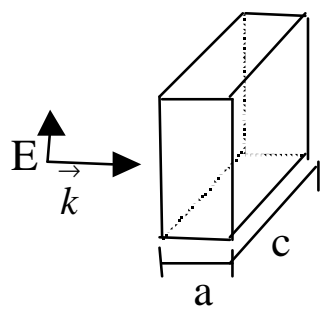

$\mathrm{E} \perp \mathrm{c}$

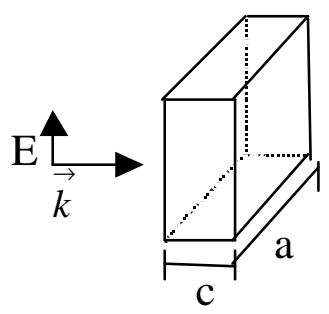

$\mathrm{E} \perp \mathrm{c}^{*}$

(a)

(b)

(c)

Figura 4.2 - Orientação das amostras investigadas em relação a polarização do feixe de prova. Onde $k$ é o vetor de onda.

Na Figura 4.3 é mostrado uma curva transiente para o cristal LiSAF dopado com $1 \%$ de $\mathrm{Cr}^{3+}$, cujo formato da curva é característico de ds/dT positivo. A excitação foi realizada utilizando um feixe laser de $\mathrm{Ar}^{+}$sintonizado em 488nm e 
fixo com polarização $E \perp c$. Os quadrados fechados representam a medida realizada na configuração (a), e círculos abertos na configuração (b).

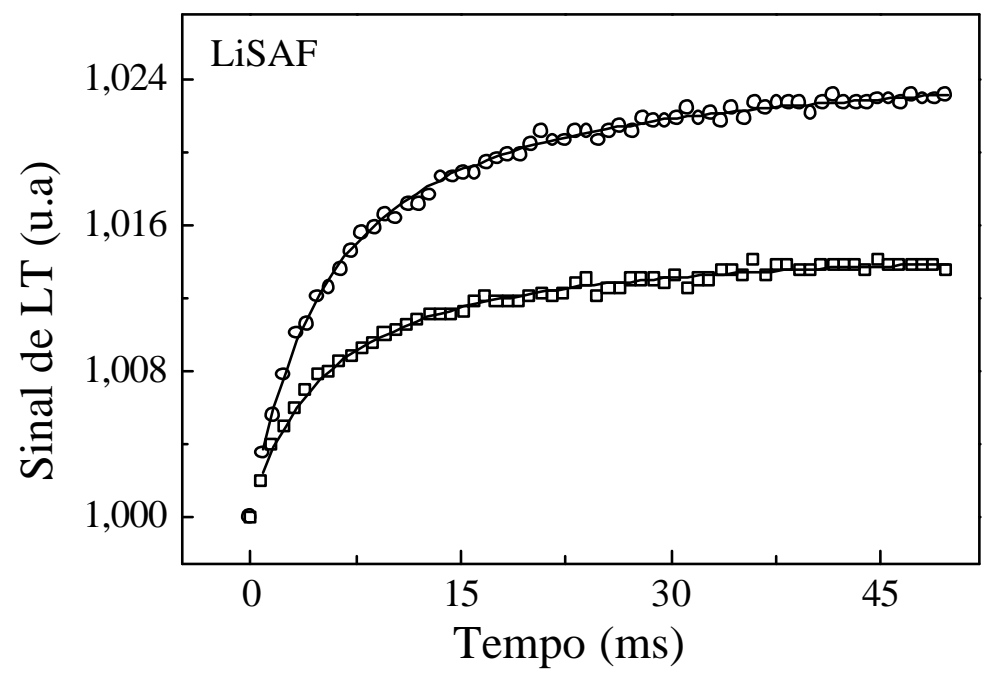

Figura 4.3 - Sinal de LT no cristal LiSAF com $1 \%$ de $\mathrm{Cr}^{3+}$. Feixe de excitação $\left(\lambda_{\text {exc }}=488 \mathrm{~nm}\right)$ com polarização $E \perp c$. (o) feixe de prova $\left(?_{p}=632 \mathrm{~nm}\right)$ polarizado de acordo com configuração $(a)$ e $(\cap)$ configuração $(b)$ da Figura 4.2, respectivamente. Ajuste teórico feito com a Eq. (2.3). Com $w=4,15 \times 10^{-3} \mathrm{~cm}^{2}, V=1,73, \quad m=19,2$ e $t_{c}=(0,58 \pm 0,01) \times 10^{-3} \mathrm{~s}$ para ambas as curvas.

Na Figura 4.4 é mostrado a linearidade da amplitude do sinal de LT com a potência do feixe de excitação, $P$. Os dados da Figura 4.4 foram ajustados com uma função linear, ?/AL $\mathrm{Aff}_{\mathrm{f}}=\mathrm{a}+\mathrm{bP}$, onde $\mathrm{a}=-0,008 \pm 0,007$ e $\mathrm{b}=0,39 \pm 0,01$ para a medida com o laser de prova $E \perp c$ (curva com círculos abertos) e a $=-0,02 \pm 0,007$ e $\mathrm{b}=0,27 \pm 0,01$ para $E / / c$ (curva com quadrados fechados). Como a difusão de calor ocorre em ambas as direções, paralela e perpendicular ao eixo $c$, então para obter ds/dT da Eq. (2.5), foi tomado o valor médio $\langle k\rangle=\left(\mathrm{k}_{\perp}+\mathrm{k}_{/ /}\right) / 2$. Desde que

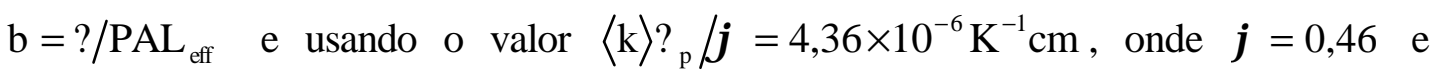
$\langle\mathrm{k}\rangle=31,5 \times 10^{-3} \mathrm{~W} / \mathrm{Kcm}$, nós calculamos $\mathrm{ds} / \mathrm{dT}_{\perp c}=(1,7 \pm 0,04) \times 10^{-6} \mathrm{~K}^{-1} \quad \mathrm{e}$ $\mathrm{ds} / \mathrm{dT}_{/ / \mathrm{c}}=(1,18 \pm 0,04) \times 10^{-6} \mathrm{~K}^{-1}$. 


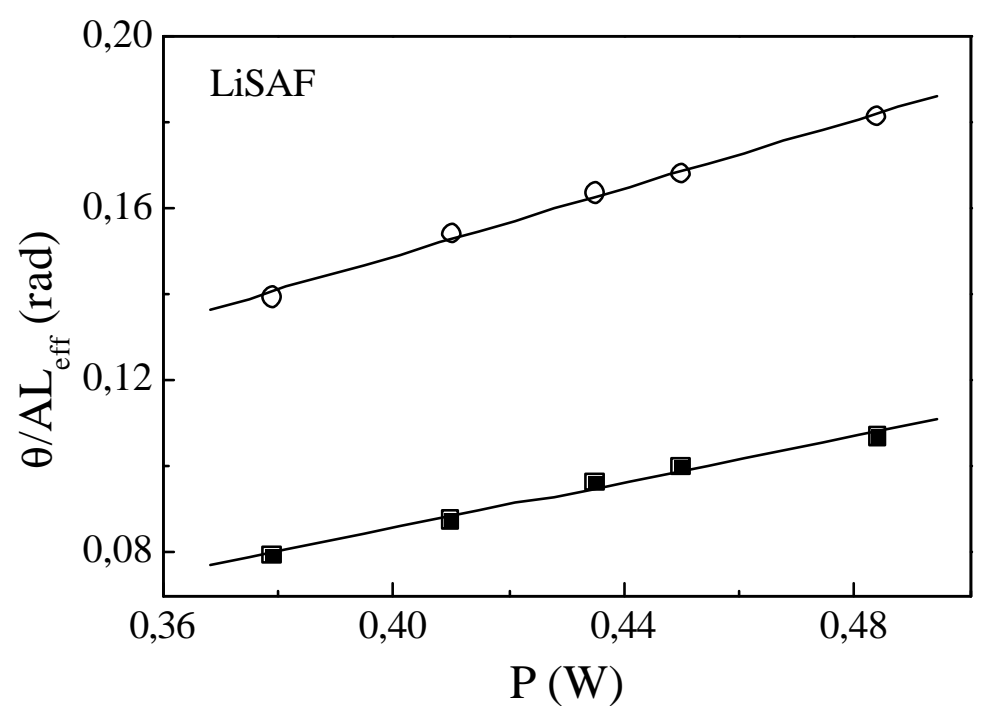

Figura 4.4 - Medidas de LT no cristal LiSAF com o feixe de prova $\left(?_{p}=632 \mathrm{~nm}\right)$ polarizado. (-) representa a medida feita com o laser de prova com polarização paralela ao eixo c da amostra e (o) com polarização perpendicular ao eixo $c$.

Quando a medida foi realizada de acordo com a configuração (c), observamos uma inversão no sinal de LT (Figura 4.5). Isto significa que neste configuração $\mathrm{ds} / \mathrm{dT}$ é negativo. $\mathrm{O}$ valor obtido foi $\mathrm{ds} / \mathrm{dT}_{\perp c^{*}}=-(1,86 \pm 0,06) \times 10^{-6} \mathrm{~K}^{-1}$.

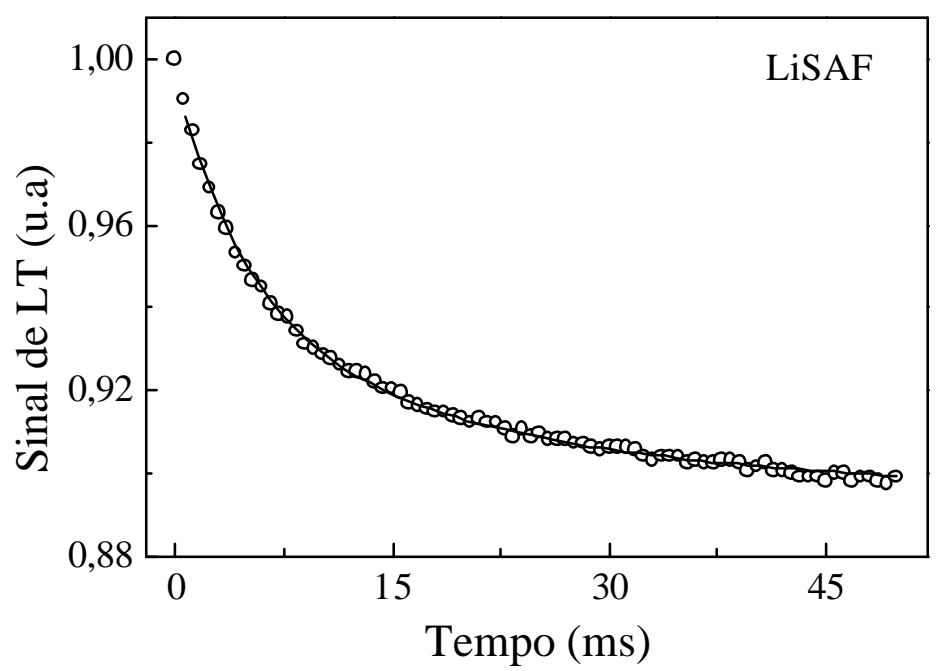

Figura 4.5 - Sinal de LT no cristal LiSAF com $1 \%$ de $\mathrm{Cr}^{3+}$. Feixe de excitação $\left(\lambda_{\text {exc }}=488 \mathrm{~nm}\right)$ com polarização $E \perp c$ e feixe de prova $\left(?_{p}=632 \mathrm{~nm}\right)$ polarizado de acordo com a configuração (a). Ajuste teórico feito com a Eq. (2.3). $w_{e}=4,15 \times 10^{-3} \mathrm{~cm}^{2}$, $t_{c}=(0,70 \pm 0,01) \times 10^{-3} \mathrm{~s} e ?=(11,23 \pm 0,02) \times 10^{-2}$. 
No nosso experimento o feixe de excitação sempre se propagou perpendicularmente ao eixo $c$, com exceção da direção representada na Figura 4.2 (c). Portanto, o termo $?_{\alpha}$ foi calculado usando $\alpha_{\perp}$, desde que este termo está relacionado a expansão érmica ao longo do eixo $c$. Similarmente a tensão ("stress") é devido ao perfil de temperatura radial, tal que no cálculo de $\Delta_{q}$ foi considerado $\langle\mathrm{a}\rangle=\left(\mathrm{a}_{\perp}+\mathrm{a}_{/ /}\right) / 2$. No caso da configuração (c), como o feixe de excitação se propaga ao longo do eixo, foi considerado o valor de $\alpha$ paralelo, $\alpha_{/ /}$e para o terceiro termo o valor de $\alpha$ perpendicular, $\alpha_{\perp}$. Os resultados obtidos para os cristais uniaxiais estão sumarizados na Tabela 4.2.

\begin{tabular}{|c|c|c|c|c|c|c|}
\hline Amostras & $\begin{array}{r}\mathrm{dn} / \mathrm{dT} \\
\left(10^{-6} K^{-1}\right)\end{array}$ & $\begin{array}{c}a \\
\left(10^{-6} K^{-1}\right) ?\end{array}$ & $\begin{array}{c}\Delta_{\alpha} ? \\
\left(10^{-6} K^{-1}\right)\end{array}$ & $\begin{array}{c}\Delta_{q} \\
\left(10^{-6} K^{-1}\right)\end{array}$ & $\begin{array}{l}\mathrm{ds} / \mathrm{dT})_{\text {cal }} \\
\left(10^{-6} K^{-1}\right)\end{array}$ & $\begin{array}{l}\mathrm{ds} / \mathrm{dT})_{\mathrm{TL}} \\
\left(10^{-6} K^{-1}\right)\end{array}$ \\
\hline \multirow[t]{2}{*}{$Y V O_{4}: N d$} & 3,0 & 11,37 & 5,7 & $1,1^{(\mathrm{d})}$ & 9,8 & 15,4 \\
\hline & $8,5^{(a)}$ & $4,43^{(a)}$ & 5,7 & $1,1^{(\mathrm{d})}$ & 15,3 & 12,7 \\
\hline \multirow[t]{3}{*}{$Y L F: N d$} & $-4,3^{(b)}$ & $8,3^{(b)}$ & 8,3 & $0,25^{(\mathrm{e})}$ & 4,3 & 18 \\
\hline & $-2,0^{(b)}$ & $13,3^{(b)}$ & 8,3 & $0,25^{(\mathrm{e})}$ & 6,5 & 20 \\
\hline & --- & --- & 5,2 & $0,32^{(\mathrm{e})}$ & 3,5 & \\
\hline \multirow[t]{3}{*}{$\operatorname{LiSAF}: C r$} & $-4,0^{(b)}$ & $-10^{(b)}$ & 13,2 & $0,28^{(\mathrm{e})}$ & 9,5 & 1,2 \\
\hline & $-2,5^{(b)}$ & $25^{(b)}$ & 13,2 & $0,28^{(\mathrm{e})}$ & 11,0 & 1,7 \\
\hline & --- & --- & $-5,3$ & $0,93^{(h)}$ & $-6,8$ & $-1,86$ \\
\hline \multirow[t]{3}{*}{$L i S G a F: C r / / c$} & $-4,0^{(c)}$ & $-6^{(b)}$ & 6,76 & $0,13^{(\mathrm{e})}$ & 2,9 & 2,7 \\
\hline & $-2,5^{(\mathrm{c})}$ & $13^{(b)}$ & 6,76 & $0,13^{(\mathrm{e})}$ & 4,4 & 4,0 \\
\hline & --- & --- & $-3,12$ & $0,47^{(\mathrm{h})}$ & $-5,1$ & $-0,2$ \\
\hline
\end{tabular}

Tabela 4.2 - Propriedade das amostras estudados. (a) Ref. [96]; (b) Ref. [61]; (c) dados do LiSAF ; (d) $\Delta_{q}$ calculado com os dados do YAG ; (e) calculado com os dados do CaF 2 . $?_{a}=(n-1)(1+\mu) a$ e $?_{q}=n^{3} Y \alpha\left(q_{\perp}+q_{/ /}\right) / 4$. 


\subsection{Discussão.}

Uma vez que, para alguns materiais, os parâmetros fotoelásticos não são encontrados na literatura, para estimar a contribuição relativa do termo devido ao efeito fotoelástico, os valores para os cristais fluoretos foram estimados usando os valores do $\mathrm{CaF}_{2}$ obtidos na Ref. [97]. Para o cristal $\mathrm{YVO}_{4}$ foi usado o valor do YAG. Embora, esta aproximação seja um tanto grosseira, ela serve para dar uma idéia da contribuição do efeito fotoelástico. Para os cristais LiSAF e YLF o parâmetro dn/dT exibe um valor negativo, com exceção do LiSGaF cujo valor de dn/dT não foi encontrado na literatura; isto é uma propriedade comum de muitos cristais fluoretos [65]. Desde que a contribuição negativa de dn/dT para a lente térmica, contribui para cancelar o efeito positivo devido ao termo da expansão térmica, a lente térmica tende a ser muito menor em cristais fluoretos, quando comparados por exemplo a cristais óxidos tal como o YAG : $\mathrm{Nd}^{3+}$, onde os termos da Eq. (4.5) se somam resultando em uma forte lente térmica.

Para o $\mathrm{YVO}_{4}$ um bom acordo foi obtido para ds/dT) $)_{\perp c}$, mas para ds/dT) $)_{/ / \mathrm{c}}$ existe uma discrepância de $\sim 100 \%$. No entanto, devemos lembrar que nem todos os dados necessários para o cálculo de ds/dT, para a maioria dos materiais estudados, são encontrados na literatura. Devemos lembrar também que, as propriedades anisotrópicas dos cristais não são propriamente levadas em conta no modelo teórico da LT que nós usamos. Nós achamos que isto é provavelmente a principal razão para a divergência entre $\mathrm{ds} / \mathrm{dT})_{\text {cal }}$ e $\left.\mathrm{ds} / \mathrm{dT}\right)_{\mathrm{LT}}$ observado nos cristais uniaxiais, mostrado na Tabela 4.2. 
Em conclusão, neste capítulo nós demonstramos a potencialidade da técnica de LT para medir o valor absoluto de ds/dT em materiais óticos. Foi observado que o coeficiente de expansão térmica tem uma alta contribuição para o valor de ds/dT medido.

A soma dos termos devido ao coeficiente de expansão térmica e ao efeito fotoelástico que contribuem para $\mathrm{ds} / \mathrm{dT}$, é positivo na maioria dos materiais. Portanto, materiais com dn/dT negativo são interessantes para minimizar $|\mathrm{ds} / \mathrm{dT}|$, o qual é altamente desejável em algumas aplicações tipo laseres de vidro de alta potência, janela para laser e em algumas fibras óticas. Este trabalho pode ajudar a encontrar composições de vidros de modo a minimizar ou controlar ds/dT para aplicações especiais.

\subsection{Espectroscopia de LT}

Nos materiais onde não conhecemos o valor do calor específico e nem a condutividade térmica, $\mathrm{k}$, de tal forma que não podemos determinar $\mathrm{o}$ valor de ds/dT, que é o caso do vidro calcohaleto, é interessante escrever a Eq. (2.5) como [7]:

$$
?=\left(\frac{\mathrm{PAL}_{\text {eff }}}{\mathrm{D} ?}\right)\left(-\frac{\mathrm{ds}}{\mathrm{dQ}}\right) ; \frac{\mathrm{ds}}{\mathrm{dQ}}=\frac{1}{? \mathrm{C}} \frac{\mathrm{ds}}{\mathrm{dT}}
$$

onde ds/dQ $\left(\mathrm{cm}^{3} \mathrm{~J}^{-1}\right)$ representa a variação do caminho ótico com o calor depositado por unidade de volume.

Nós estudamos uma amostra de vidro calcohaleto $\left(40 \% \mathrm{PbI}_{2}-30 \% \mathrm{Sb}_{2} \mathrm{~S}_{3}-30 \% \mathrm{As}_{2} \mathrm{~S}_{3}\right)$ [31] que é similar ao de GaLaS com a 
diferença que sua absorção (ou "UV edge") ocorre em torno de 720nm. Ou seja, o vidro absorve totalmente $633 \mathrm{~nm}$, como pode ser visto na Figura 4.5 . Este vidro é interessante por apresentar larga janela de transparência no infravermelho $(\sim 13 \mu \mathrm{m})$ e baixa energia de fônon. Estas são características de vidros com fraca ligação entre seus constituintes (baixo $\mathrm{T}_{\mathrm{g}}$ ) que apresentam a desvantagem de terem baixa condutividade e difusividade térmicas e alto coeficiente de expansão.

Nesta amostra, devido sua alta absorção em 633nm, as medidas de LT foram realizadas na configuração de feixe único, sem a utilização do laser de $\mathrm{HeNe}$ como feixe de prova. As medidas foram realizadas usando um laser de Ti-safira, com o objetivo de investigar a dependência de ds/dQ com o comprimento de onda no intervalo de 730 a $840 \mathrm{~nm}$.

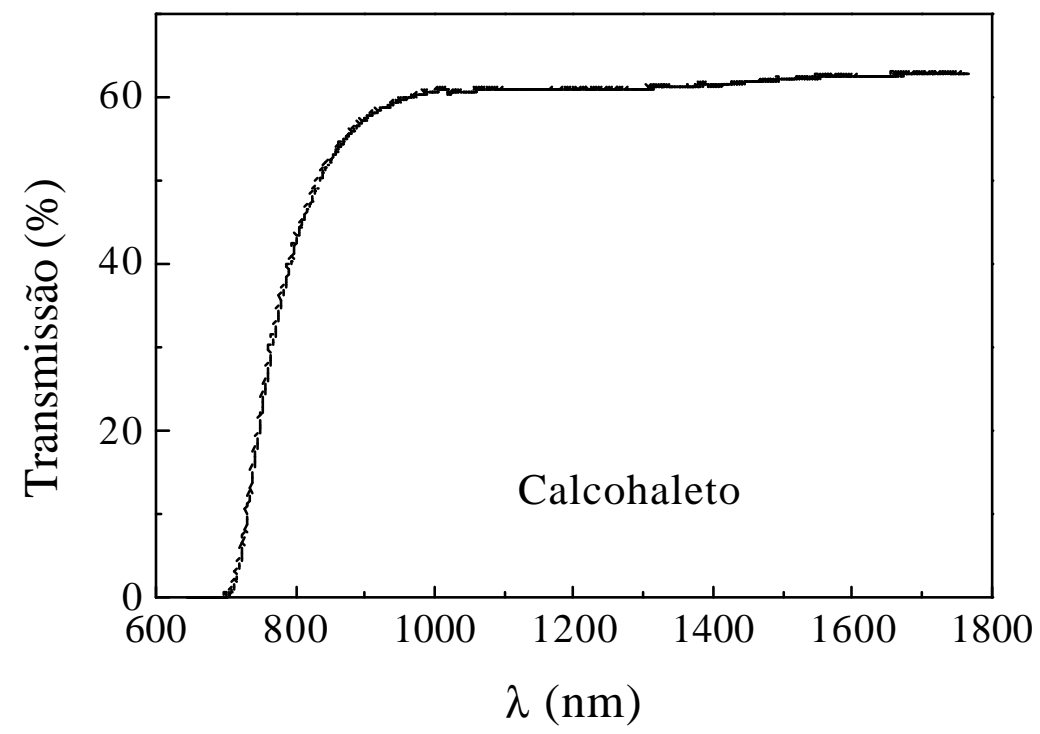

Figura 4.6 - Espectro de transmitância do vidro calcohaleto no infravermelho próximo.

A Figura 4.7 mostra o comportamento de ds/dQ e do coeficiente de absorção, $A$, em função do comprimento de onda entre 730 e 840nm (devido a alta absorção não conseguimos fazer medidas para ?<730nm). Podemos notar que 
ds/dQ e o coeficiente de absorção, A, cresce por um fator de 9,3 e 1,7, respectivamente, quando o comprimento de onda decresce de 840 para $730 \mathrm{~nm}$.

O decréscimo de ds/dQ era esperado, uma vez que os três termos na Eq. (4.5) dependem do comprimento de onda. No entanto, medidas da intensidade do laser incidente e refletido, nos possibilitou determinar a refletância, R, do vidro e através da relação $\mathrm{R}=(\mathrm{n}-2)^{2} /(\mathrm{n}+1)^{2}$, o índice de refração. Nós observamos que $n$ permanece aproximadamente constante $(\mathrm{n} \sim 2,5)$. Portanto, a variação de ds/dT foi atribuída a dependência de $\mathrm{dn} / \mathrm{dT}$ ao comprimento de onda e ou aos coeficientes de tensão, $q$. Este estudo espectrocópico é interessante, uma vez que pode ajudar a distinguir as diferentes contribuições para ds/dT .

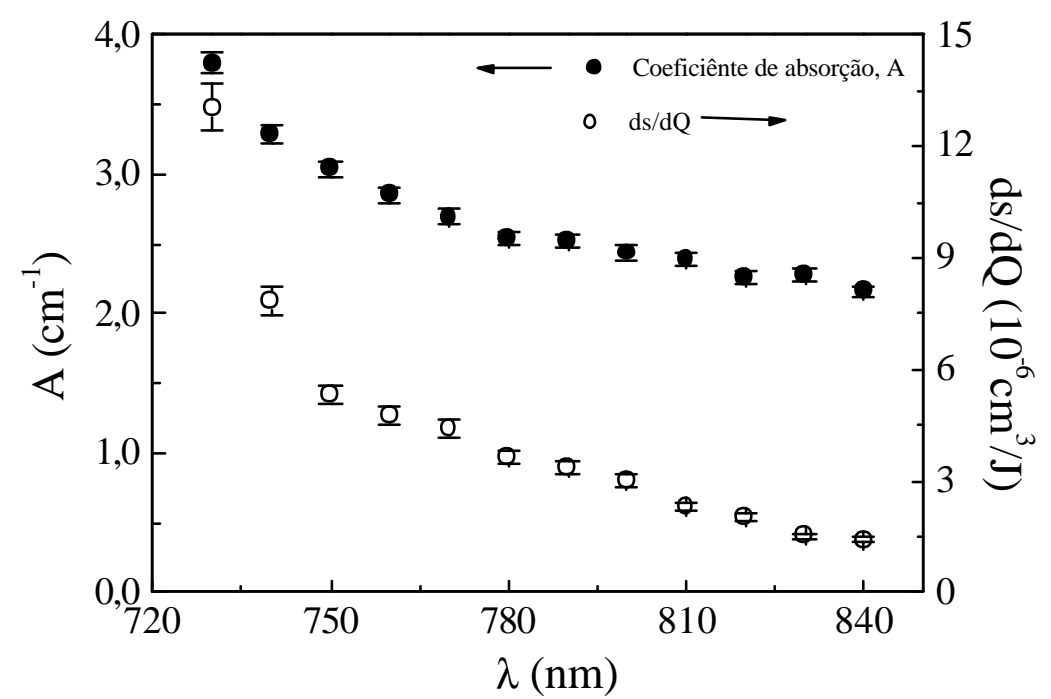

Figura 4.7 - Variação do caminho ótico com o calor depositado por unidade de volume, $d s / d Q$, (círculo aberto) e coeficiente de absorção, A, (círculo fechado) versus o comprimento de onda, $\lambda$. 


\section{Capítulo 5}

\section{Transferência de energia.}

Neste capítulo iremos apresentar a técnica de LT como uma ferramenta alternativa para medir os parâmetros de transferencia de energia em sólidos dopados, que podem ocorrer por "upconversion Auger" e relaxação cruzada. Como já discutido no Capítulo 3, o conhecimento destes parâmetros é extremamente importante, uma vez que eles são responsáveis pela diminuição da eficiência quântica em sólidos com altas concentrações de íons dopantes.

\subsection{Estudo dos mecanismos de transferência de energia nos sólidos dopados com $\mathrm{Cr}^{3+}$ e $\mathrm{Nd}^{3+}$ utilizando a técnica de LT.}

No Capítulo 3 mostramos que vários fatores podem contribuir para $\mathrm{o}$ decaimento não radiativo. Aqui iremos mostrar que, um outro processo, extremamente importante, também contribui para o decaimento não radiativo, o processo de conversão ascendente de energia "upconversion Auger" [48-50]. Neste processo ocorre uma interação entre dois íons no nível excitado $\left({ }^{4} \mathrm{~F}_{3 / 2}\right)$, como ilustrado na Figura 5.1. Estes íons interagem de tal forma que um íon $\mathrm{Nd}^{3+}$ é transferido para um nível de maior energia, enquanto o outro retorna para um estado de menor energia. O resultado final desta transferência de energia é o aumento de calor dentro da matriz que pode ser determinado pela técnica de LT. 
Doualan e colaboradores [98] calcularam o parâmetro de "upconversion" em vidros silicatos e fosfatos dopados com $\mathrm{Nd}^{3+}$, diretamente dos microparâmetros de transferência de energia obtidos pelo modelo de Dexter [99]. Payne e colaboradores [50] também mediram o parâmetro de "upconversion" em vários vidros dopados com $\mathrm{Nd}^{3+}$.

Apesar do grande número de trabalhos a respeito do parâmetro de transferência de energia em sólidos dopados, ainda não se encontra na literatura resultados experimentais destes parâmetros em função da concentração para um mesmo conjunto de amostras.

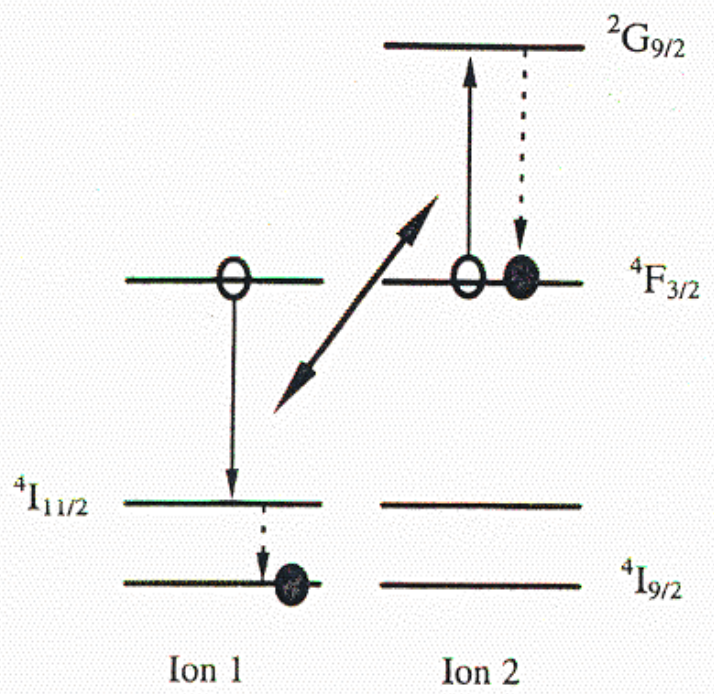

(a)

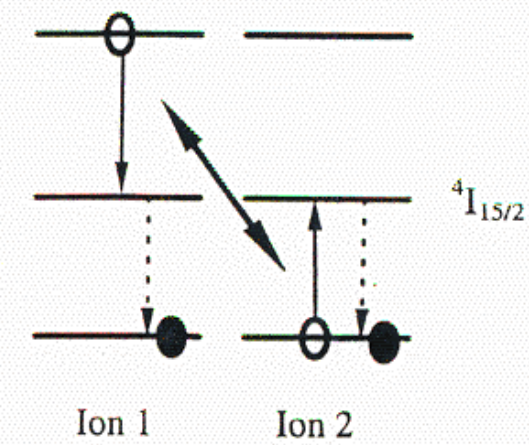

(b)

Figura 5.1 - Diagrama esquemático dos processos de transferência de energia no $\mathrm{Nd}^{3+}$ (a) "Upconversion Auger" (b) Relaxação cruzada. Figura tirada da Ref. [49].

A dinâmica do nível ${ }^{4} \mathrm{~F}_{3 / 2}$, considerado os processos "upconversion Auger" e relaxação cruzada, pode ser descrita pela equação de taxa

$$
\frac{\mathrm{dN}}{\mathrm{dt}}=\frac{\mathrm{s} I}{\mathrm{~h} ?} \mathrm{~N}_{\mathrm{g}}-\frac{\mathrm{N}_{\mathrm{ex}}}{\mathrm{t}_{0}}-?_{\text {up }} \mathrm{N}_{\mathrm{ex}}^{2}-?_{\mathrm{rc}} \mathrm{N}_{\mathrm{ex}} \mathrm{N}_{\mathrm{g}}
$$


onde $\mathrm{N}_{\mathrm{ex}}$ é a população do nível excitado ${ }^{4} \mathrm{~F}_{3 / 2}, \quad \mathrm{~N}_{\mathrm{g}}$ a população do nível fundamental ${ }^{4} \mathrm{I}_{9 / 2}, \sigma$ a seção de choque de absorção, I a intensidade do laser de excitação, $h$ ? a energia do fóton de excitação, $\mathrm{t}_{0}$ é o tempo de vida medido na amostra de baixa concentração. Os parâmetros $?_{\text {up }}$ e $?_{\text {rc }}$ são os parâmetros de "upconversion Auger" e relaxação cruzada, respectivamente. Assumindo que a população total é $\mathrm{N}_{0}=\mathrm{N}_{\mathrm{g}}+\mathrm{N}_{\mathrm{ex}}$ e $\mathrm{n}_{\mathrm{ex}}=\mathrm{N}_{\mathrm{ex}} / \mathrm{N}_{0}$, a Eq. (5.1) pode ser rescrita

$$
\frac{\mathrm{dn}}{\mathrm{ex}}=\frac{\mathrm{s} I}{\mathrm{~h} ?}\left(1-\mathrm{n}_{\mathrm{ex}}\right)-\mathrm{n}_{\mathrm{ex}}\left(\frac{1}{\mathrm{t}_{0}}+?_{\mathrm{rc}} \mathrm{N}_{0}\right)-\mathrm{n}_{\mathrm{ex}}^{2} \mathrm{~N}_{\mathrm{o}}\left(?_{\mathrm{up}}-?_{\mathrm{rc}}\right)
$$

$\mathrm{ou}$

com

$$
\frac{\mathrm{dn}_{\mathrm{ex}}}{\mathrm{dt}}=\frac{\mathrm{S}}{\mathrm{t}}-\frac{\mathrm{n}_{\mathrm{ex}}}{\mathrm{t}}(1+\mathrm{S})-\frac{\mathrm{n}_{\mathrm{ex}}^{2}}{\mathrm{t}} \beta
$$

$$
\mathrm{t}^{-1}=\left(\mathrm{t}_{0}^{-1}+?_{\mathrm{rc}} \mathrm{N}_{0}\right)
$$

$\mathrm{e}$

$$
\beta=\mathrm{N}_{0} \mathrm{t}\left(?_{\text {up }}-?_{\text {rc }}\right)
$$

onde $S=I(r) / I_{s}$ é o parâmetro de saturação, $I_{s}=h$ ?/st a intensidade de saturação. Para obter o parâmetro $\mathrm{S}$, devemos considerar a intensidade média ao longo da amostra e da direção radial, tal que $\mathrm{S}_{0}=\langle\mathrm{I}\rangle / \mathrm{I}_{\mathrm{s}}$, sendo $\langle\mathrm{I}\rangle=\mathrm{I}_{0} \mathrm{~L}_{\text {eff }} / 2 \mathrm{~L}$, $\mathrm{I}_{0}=2 \mathrm{P} / \pi \mathrm{w}^{2}$. É importante lembrar também que, no cálculo da intensidade devemos levar em conta a reflexão da amostra (R). De tal forma que teremos uma intensidade média dada por $\langle\mathrm{I}\rangle=\mathrm{P}(1-\mathrm{R}) \mathrm{L}_{\text {eff }} /\left(\mathrm{Lp} \mathrm{w}^{2}\right)$.

Nota-se que a Eq. (5.4) é similar a Eq. (3.3) com

$$
?_{\mathrm{rc}}=\frac{\mathrm{N}_{0}^{\mathrm{p}-1}}{\mathrm{t}_{0} \mathrm{~N}_{\mathrm{cq}}^{\mathrm{p}}}
$$


ou seja, para os nossos vidros, como obtivemos $\mathrm{p} \approx 2$ (vide Capítulo 3) $?_{\mathrm{rc}}=\mathrm{N}_{0} \mathrm{t}_{0}^{-1} \mathrm{~N}_{\mathrm{cq}}^{-2}$.

A Eq. (5.3) pode ser usada para estudar a evolução de $\mathrm{n}_{\mathrm{ex}}(\mathrm{t})$ tanto na excitação quanto no decaimento. No caso de decaimento, $\mathrm{S}=0$, a solução da Eq. (5.3) fica

$$
\mathrm{n}_{\mathrm{ex}}(\mathrm{t})=\frac{\mathrm{n}_{\mathrm{ex}}(0) \mathrm{e}^{-\mathrm{t} / \mathrm{t}}}{1+\beta \mathrm{n}_{\mathrm{ex}}(0)\left(1-\mathrm{e}^{-\mathrm{t} / \mathrm{t}}\right)}
$$

onde em $\mathrm{t}=0 \mathrm{n}_{\mathrm{ex}}(\mathrm{t})=\mathrm{n}_{\mathrm{ex}}(0)$.

No caso de excitação, $S \neq 0$, a solução para a Eq. (5.3) no estado estacionário é dada por:

$$
\mathrm{n}_{\mathrm{ex}}=\frac{\mathrm{N}_{\mathrm{ex}}}{\mathrm{N}_{\mathrm{o}}}=\frac{-(\mathrm{S}+1) \pm \sqrt{(\mathrm{S}+1)^{2}+4 \mathrm{~S} \beta}}{2 \beta}
$$

A Figura 5.2 mostra uma simulação do comportamento da população do estado excitado considerando os processos "upconversion Auger" e relaxação cruzada, em função do parâmetro de saturação S. Podemos observar que, para altos valores do parâmetro B (vide Eq. (5.5)), há uma forte depopulação do estado excitado. No caso de não haver perdas devido aos processos de "upconversion Auger" e relaxação cruzada $(\beta=0)$, a depopulação do estado excitado é devido somente a saturação. Esta simulação nos mostra a grande importância do conhecimento destes processos de transferência de energia, pois eles limitam o ganho e a potência de saída de laseres e amplificadores. 


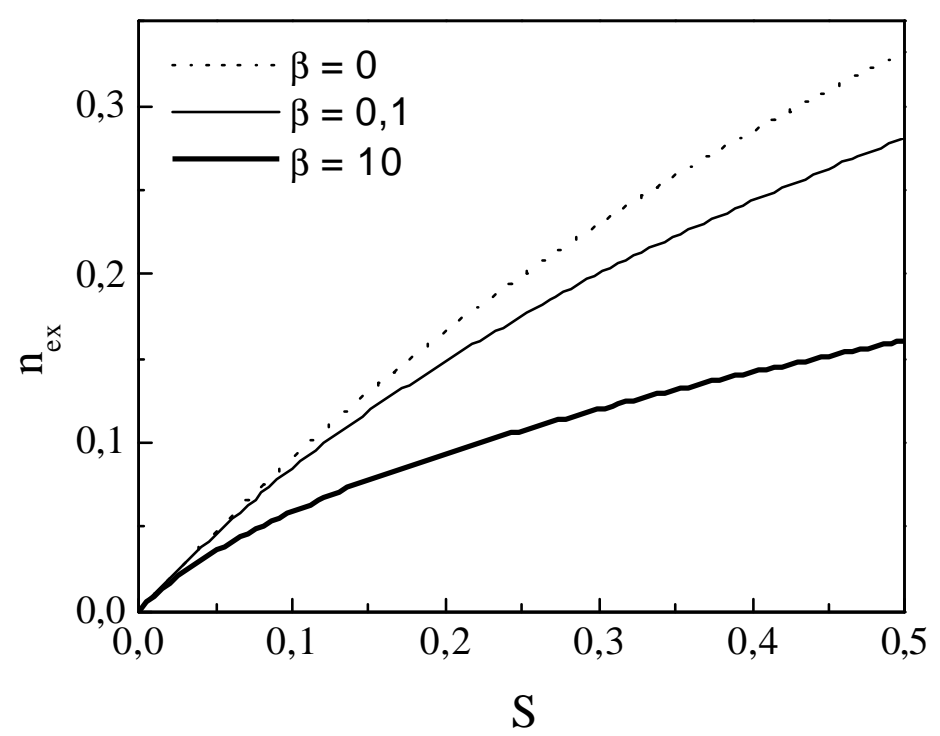

Figura 5.2 - Simulação da fração de população do estado excitado em função do parâmetro do saturação $S$, para vários valores do parâmetro de perda $\beta$.

No Capítulo 3 mostramos que a eficiência quântica de um dado nível de energia é definida por $?=\mathrm{t}_{\exp } / \mathrm{t}_{\mathrm{R}}$ (vide Eq. (3.1)). Na ausência de qualquer processo de transferência de energia (amostra de baixa concentração), nós definimos a eficiência quântica como sendo $?_{0}=\mathrm{t}_{0} / \mathrm{t}_{\mathrm{R}}$ (vide Eq. (3.6)). Na presença dos processos de "upconversion Auger" e relaxação cruzada, a expressão da eficiência quântica deve ser mudada devido as novas perdas e nós temos que:

$$
?=\frac{\mathrm{t}_{\mathrm{R}}^{-1}}{\mathrm{t}_{0}^{-1}+?_{\mathrm{up}} \mathrm{N}_{\mathrm{ex}}+?_{\mathrm{rc}} \mathrm{N}_{\mathrm{g}}}=\frac{?_{\mathrm{lp}}}{1+\beta \mathrm{n}_{\mathrm{ex}}}
$$

onde $?_{{ }_{\mathrm{p}}}=\mathrm{t} / \mathrm{t}_{\mathrm{R}}$ é a eficiência quântica medida em baixa potência de bombeio $\left(\mathrm{n}_{\mathrm{ex}} \rightarrow 0\right)$ e $\mathrm{n}_{\mathrm{ex}}$ é dado pela Eq. (5.8). Como podemos observar na Figura 5.2, com o aumento da potência de excitação, ocorre um aumento da população do estado excitado e esta deixa de ser desprezível, de forma que a eficiência quântica é reduzia devido ao fator $1+\beta_{\text {ex }}$ na Eq. (5.9). Esta redução na eficiência quântica, resulta no aumento no calor gerado dentro da matriz hospedeira. A expressão para o sinal de LT 
T (Eq.(3.4)) na presença dos processo "upconversion Auger" e relaxação cruzada, pode ser agora escrita como:

$$
\mathrm{T}=-\frac{1}{?_{\mathrm{p}} \mathrm{K}} \frac{\mathrm{ds}}{\mathrm{dT}}\left[1-\frac{?_{\mathrm{lp}}\left\langle ?_{\mathrm{em}}^{-1}\right\rangle ?_{\mathrm{exc}}}{1+\frac{1}{2}\left(\sqrt{(1+\mathrm{S})^{2}+4 \mathrm{~S} \beta}-(1+\mathrm{S})\right)}\right]
$$

Esta equação mostra que o sinal de $\mathrm{LT} \mathrm{T}$ em função do parâmetro de saturação S, tem uma forte dependência com os parâmetros de "upconversion Auger" e relaxação cruzada. Podemos observar que com o aumento do parâmetro ß há um significativo aumento no sinal de LT, tal como mostrado na Figura 5.3.

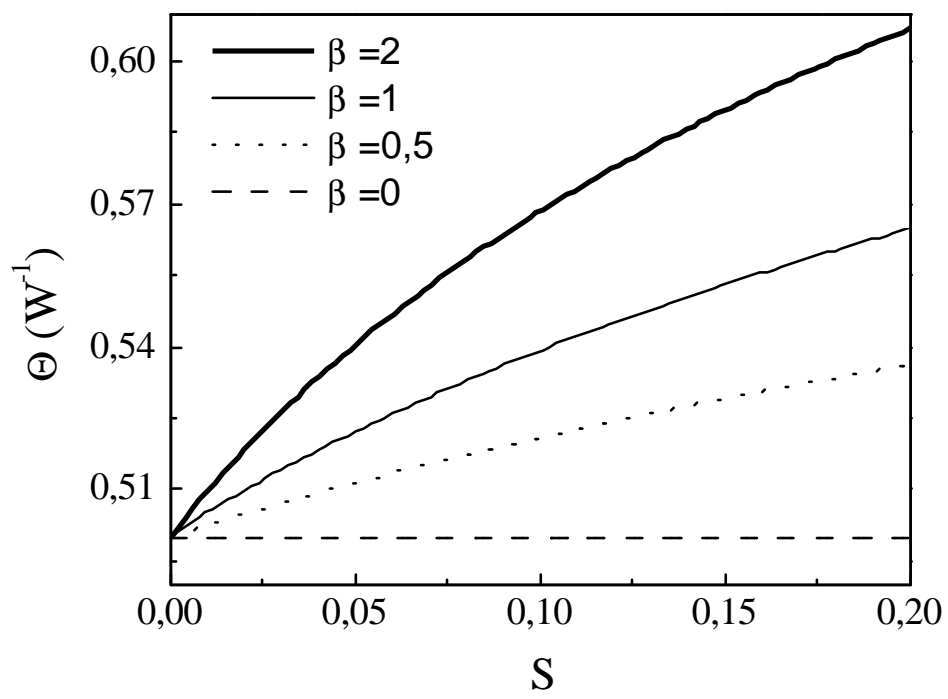

Figura 5.3 - Simulações teórica do sinal de LT normalizado versus o parâmetro de saturação $\mathrm{S}$ para vários valores de $ß$ onde $T=$ ?/PAL $L_{\text {eff }}$. Para estas simulações foram adotados $\left(1 / k ?_{p}\right) d s / d T=1$ e $?_{\text {exc }}\left\langle ?_{\text {em }}^{-1}\right\rangle=0,5$.

\subsection{Medidas de "upconversion Auger" no cristal LiSAF dopado com $\mathrm{Cr}^{3+}$.}

O primeiro estudo do efeito da transferência de energia por "upconversion Auger" utilizando a técnica de LT, foi feito recentemente no nosso grupo em cristais fluoretos dopados com $\mathrm{Cr}^{3+}$ [100]. $\mathrm{O} \mathrm{Cr}^{3+}$ no cristal de LiSAF funciona como um 
sistema de três níveis, de tal modo que não existe o processo de relaxação cruzada, ou seja, $?_{\mathrm{rc}}=0$, como pode ser visto na Figura 5.4. Neste caso, a depopulação do estado excitado é devido somente ao processo de "upconversion Auger", de modo que $B=?{ }_{\text {up }} \mathrm{N}_{0}$ t (vide Eq. (5.5)).

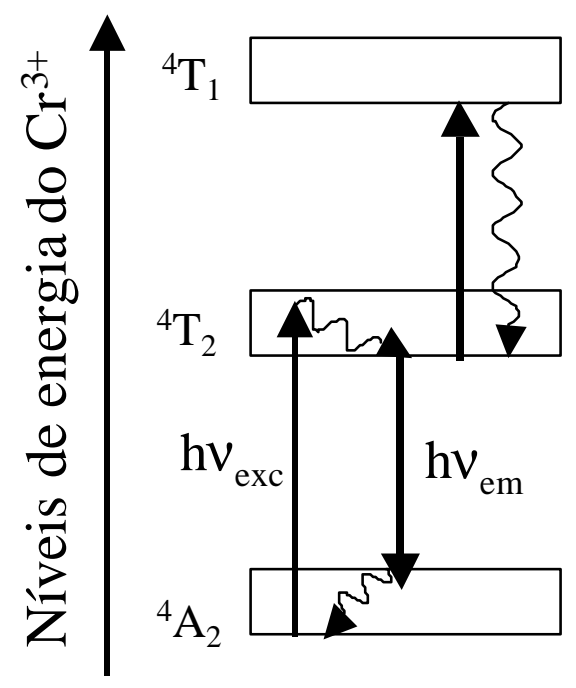

Figura 5.4 - Níveis de energia do $\mathrm{Cr}^{3+}$. Sendo $h ?_{\text {exc }}$ a energia do fóton de excitação e $h ?_{\text {em }}$ a energia do fóton emitido.

Para determinar o parâmetro $?_{\text {up }}$ do LiSAF, nós realizamos medidas de LT em função da potência de bombeio. Como fonte de bombeio nós utilizamos um laser de $\mathrm{Ar}^{+}$sintonizado em 488nm e como feixe de prova, um laser $\mathrm{HeNe}$ de baixa intensidade sintonizado em $632 \mathrm{~nm}$ [6]. A montagem experimental é a mesma apresentada no Capítulo 2. Como o cristal apresenta eixo de simetria, as medidas foram realizadas na direção perpendicular ao eixo c. Nesta direção o cristal apresenta uma maior absorção (vide Figura 5.5), o que facilita a obtenção do sinal de LT e minimiza erro nas medidas relacionados com a determinação do coeficiente de absorção. 


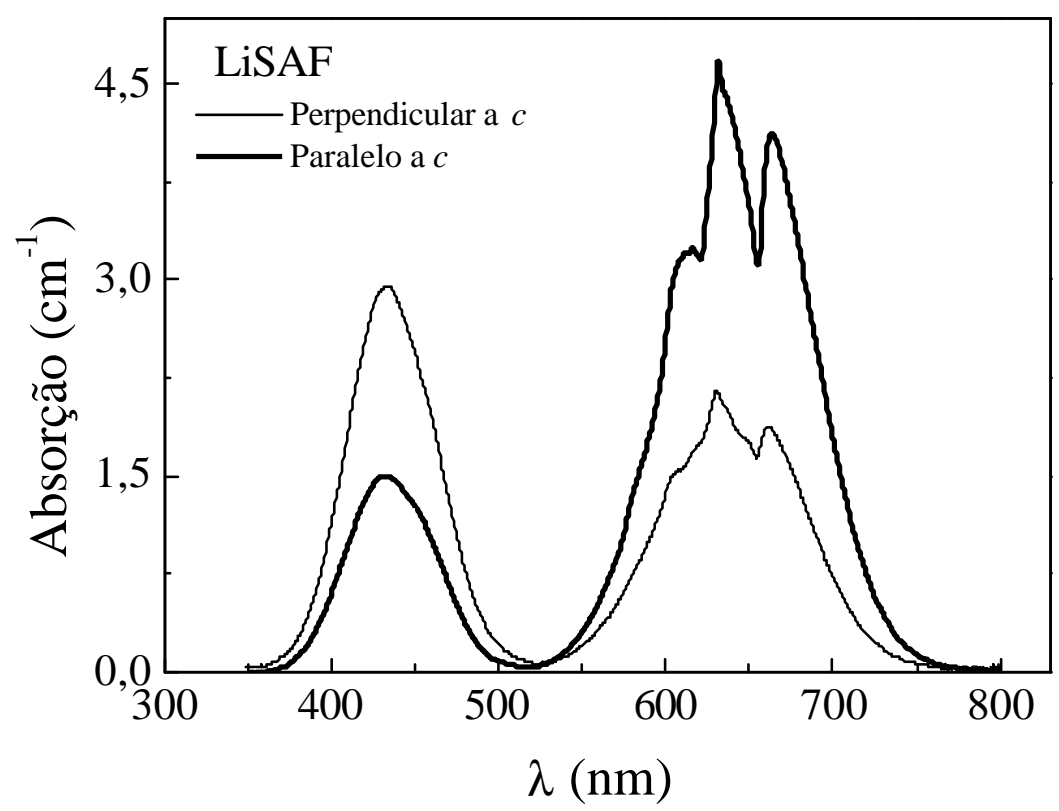

Figura 5.5 - Espectro de absorção do LiSAF com $1 \% \mathrm{Cr}^{3+}$. O espectro mostra a absorção nas direções paralela e perpendicular ao eixo $c$. $L=3,2 \mathrm{~mm}$.

Na Figura 5.6 são apresentadas as amplitudes do sinal de LT, proporcionais a ?, em função da potência do laser de bombeio. As medidas foram realizadas em duas amostras dopadas com 1 e $7 \%$ de $\mathrm{Cr}^{3+}$. Lembramos que o parâmetro ? é obtido a partir do ajuste com a Eq.(2.3). O comportamento não linear de ? $\times$ P observado particularmente para a amostra mais dopada, indica um aumento do calor gerado na amostra, que é causado pelo processo de decaimento não-radiativo devido ao efeito de "upconversion Auger".

Para interpretação destes resultados, é mais conveniente usar o parâmetro

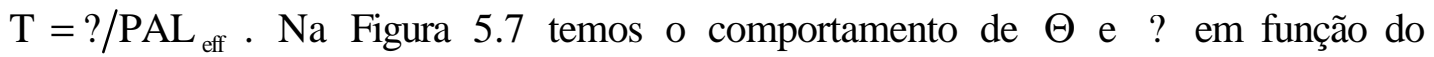
parâmetro de saturação $S$. Os dados experimentais da Figura 5.7 (a ) foram ajustados com a Eq. (5.10), de onde obtivemos os parâmetros $\beta$ e $T_{0}$, onde $T_{0}$ é o valor de $\mathrm{T}$ na ausência de transferência de energia $(\beta S \rightarrow 0)$. Os valores de $\beta$ 
obtidos foram $\beta=6,04$ e $\beta=76,3$ para as amostras de 1 e $7 \%$, respectivamente. A Figura 5.7 (b) foi obtida a partir dos valores de $\beta$ e fazendo uso da Eq. (5.9).

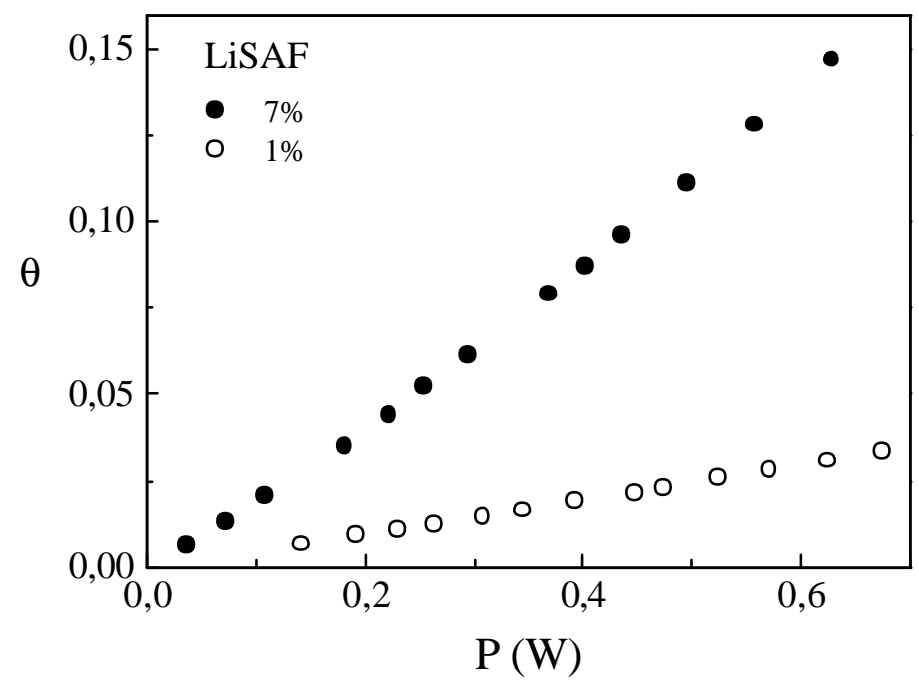

Figura 5.6 - Sinal de LT para diferentes potências do feixe de excitação em $\lambda_{\text {exc }}=488 \mathrm{~nm}$, para o LiSAF com $7 \%\left(L=1,4 \mathrm{~mm}\right.$ e $\left.A=3,37 \mathrm{~cm}^{-1}\right)$ e com $1 \%$ $\left(L=2,4 m m\right.$ e $\left.A=0,66 \mathrm{~cm}^{-1}\right)$.
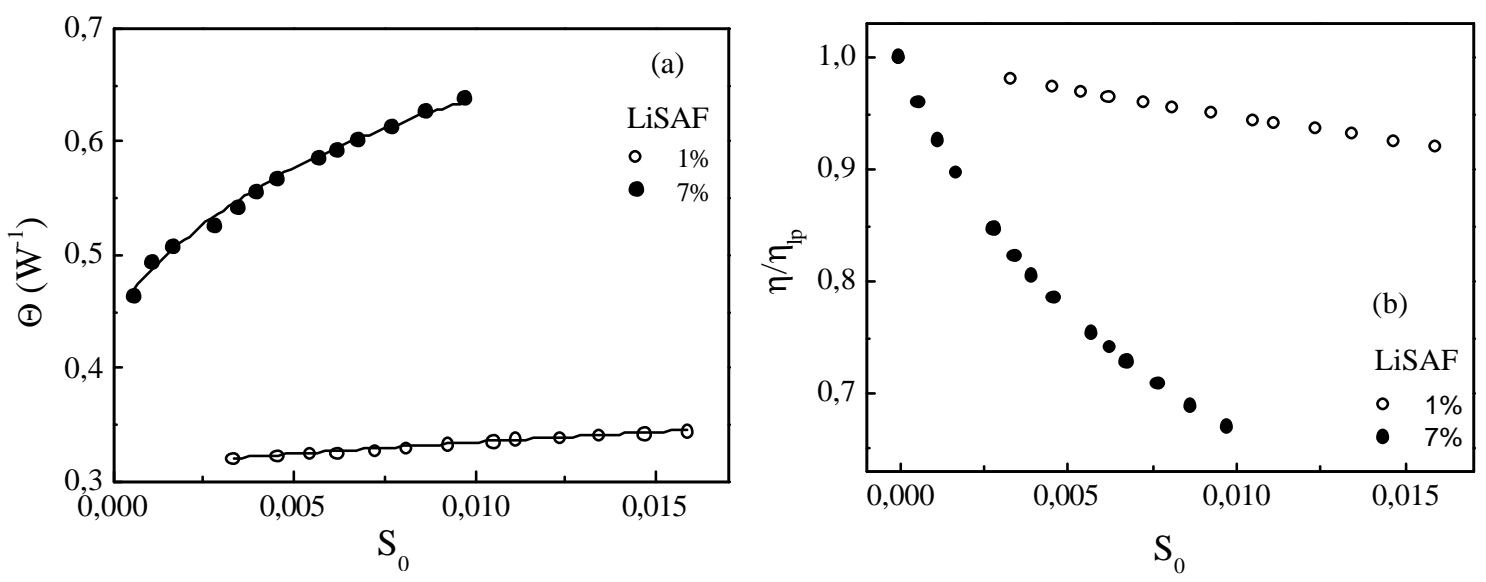

Figura 5.7 - (a) $\Theta$ em função do parâmetro de saturação $S_{0}$. Os valores de $T_{0}$ são $0,31 \pm 0,01$ e 0,44 0,01 para 1 e 7\%, respectivamente. Ajuste realizado com a Eq.(5.10). (b) Eficiência quântica em função de $S_{0}$.

O comportamento mostrado na Figura 5.7 (a) pode ser quantitativamente explicado considerando o limite para baixa excitação $(S \rightarrow 0)$, onde pela Eq. (5.8) $\mathrm{n}_{\mathrm{ex}} \sim \mathrm{S}(1-\beta \mathrm{S})$ tal que pela Eq.(5.9) ? ? ${ }_{0}\left[1-\beta S+(\beta S)^{2}\right]$. Portanto para $\beta \mathrm{S}<<1$, ? decresce linearmente com $S$, como observado para a amostra com $1 \% \mathrm{Cr}^{3+}$. No 
entanto, para a amostra com $7 \% \mathrm{Cr}^{3+}, \beta$ é aproximadamente 13 vezes maior do que da amostra com $1 \% \mathrm{Cr}^{3+}$, tal que o comportamento de $\mathrm{T}$ é parabólico com concavidade positiva, como previsto pelas simulações teórica, Figura 5.3.

Na Tabela 5.1 são comparados os valores de $?_{\text {up }}$ obtidos neste trabalho, com os valores obtidos pela primeira vez em nosso grupo por Pilla [100].

\begin{tabular}{ccccc}
\hline \hline Cristal & $\mathrm{Cr}^{3+}$ & $?_{\text {up }}$ & Método & Ref. \\
LiSAF & $(\%)$ & $\left(10^{-16} \mathrm{~cm}^{3} / \mathrm{s}\right)$ & & \\
\hline \hline & 1 & 10 & LT & Este trabalho \\
& 1 & 19,4 & LT & \\
\hline 1 & 10,8 & LT & Pilla [100] \\
& 1 & 19,2 & LT & Pilla [100] \\
\hline
\end{tabular}

Tabela 5.1 - Resultados obtidos de ? up para o LiSAF.

\subsection{Medidas de transferência de energia em sólidos dopados com $\mathrm{Nd}^{3+}$.}

De maneira análoga as medidas realizadas no item anterior, nós estudamos o efeito dos processos de transferencia de energia nos fluoretos: fluorozirconato (ZBLAN) e fluoroindato (PGIZCa ) com diferentes concentrações de $\mathrm{Nd}^{3+}$ e nos cristais: YLF e YAG .

Na Figura 5.8 temos as amplitudes do sinal de LT normalizada pela espessura efetiva da amostra, $\mathrm{L}_{\text {eff }}$, em função da potência de bombeio para um conjunto de amostras de ZBLAN dopadas com 0,5, 1,0, 2,0 e 3,0\% de $\mathrm{Nd}^{3+}$. As medidas foram realizadas com um laser de Ti:safira sintonizado em $?_{\text {exc }}=797 \mathrm{~nm}$.Na Figura 5.9 temos o comportamento $\mathrm{T}$ em função do parâmetro de saturação $\mathrm{S}$. Os valores 
dos parâmetros $\beta$ e $\mathrm{T}_{0}$ determinados a partir do ajuste teórico com a Eq.(5.10), estão apresentados na Tabela 5.3.

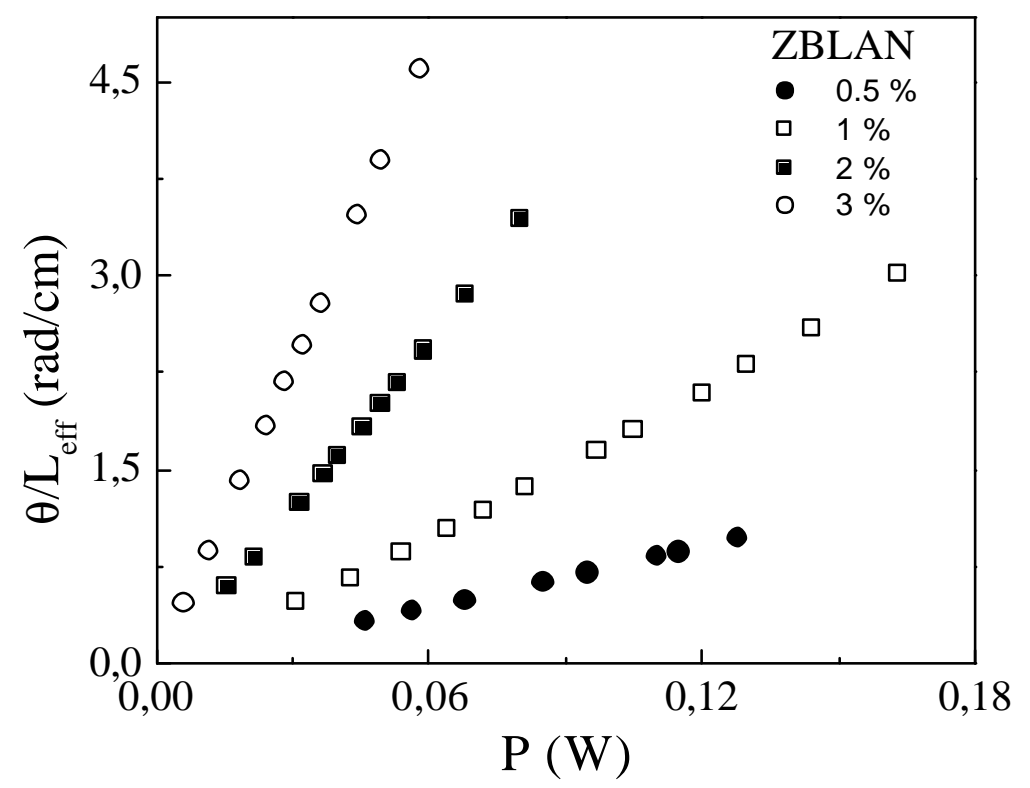

Figura 5.8 - Sinal de LT normalizado por $L_{\text {eff }}$ em função da potência do feixe de excitação em $?_{\text {exc }}=797 \mathrm{~nm}$, no vidro fluorozirconato dopado com $\mathrm{Nd}^{3+}$ (ZBLAN).

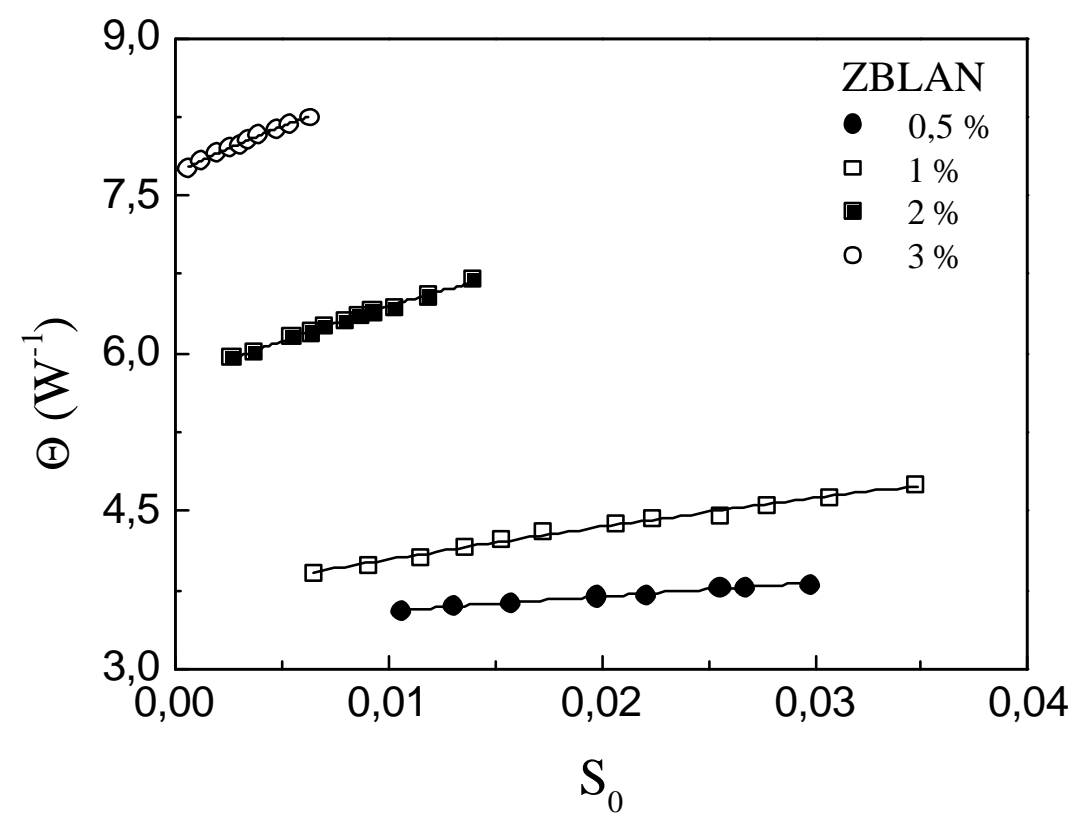

Figura 5.9 - $T$ em função do parâmetro de saturação $S_{0}$. Ajuste realizado com a Eq.(5.10). 


\begin{tabular}{ccccccc}
\hline \hline $\begin{array}{c}\text { Fluorozirconato } \\
(\text { ZBLAN })\end{array}$ & $\begin{array}{c}\mathrm{N}_{0} \\
\left(10^{20} \mathrm{~cm}^{-3}\right)\end{array}$ & $\begin{array}{c}\tau \\
(\mu s)\end{array}$ & $\begin{array}{c}\mathrm{L} \\
(\mathrm{cm})\end{array}$ & $\begin{array}{c}\mathrm{I}_{\mathrm{s}} \\
\left(\mathrm{KW} / \mathrm{cm}^{2}\right)\end{array}$ & $\mathrm{T}_{0}$ & $\beta$ \\
\hline \hline $0,5 \%$ & 0,9 & 515 & 0,23 & 24,0 & 3,4 & 1,4 \\
$1,0 \%$ & 1,9 & 460 & 0,076 & 27,0 & 3,7 & 3,7 \\
$2,0 \%$ & 3,5 & 380 & 0,076 & 32,4 & 5,7 & 10,2 \\
$3,0 \%$ & 5,2 & 260 & 0,08 & 48,0 & 7,8 & 15,9
\end{tabular}

Tabela 5.2 - Resultados do processo de transferência de energia em função da concentração de $\mathrm{Nd}^{3+}$ no vidro fluorozirconato (ZBLAN).

Usando os valores do parâmetro $B$ e a Eq. (5.9), nós graficamos o comportamento da eficiência quântica ? em função do parâmetro de saturação S, cujo resultado está apresentado na Figura 5.10. As curvas da eficiência quântica foram normalizadas pelo valor da eficiência quântica $\left(?_{\mathrm{lp}}\right)$ medida em baixa potência de bombeio $\left(\mathrm{n}_{\mathrm{ex}} \rightarrow 0\right)$.

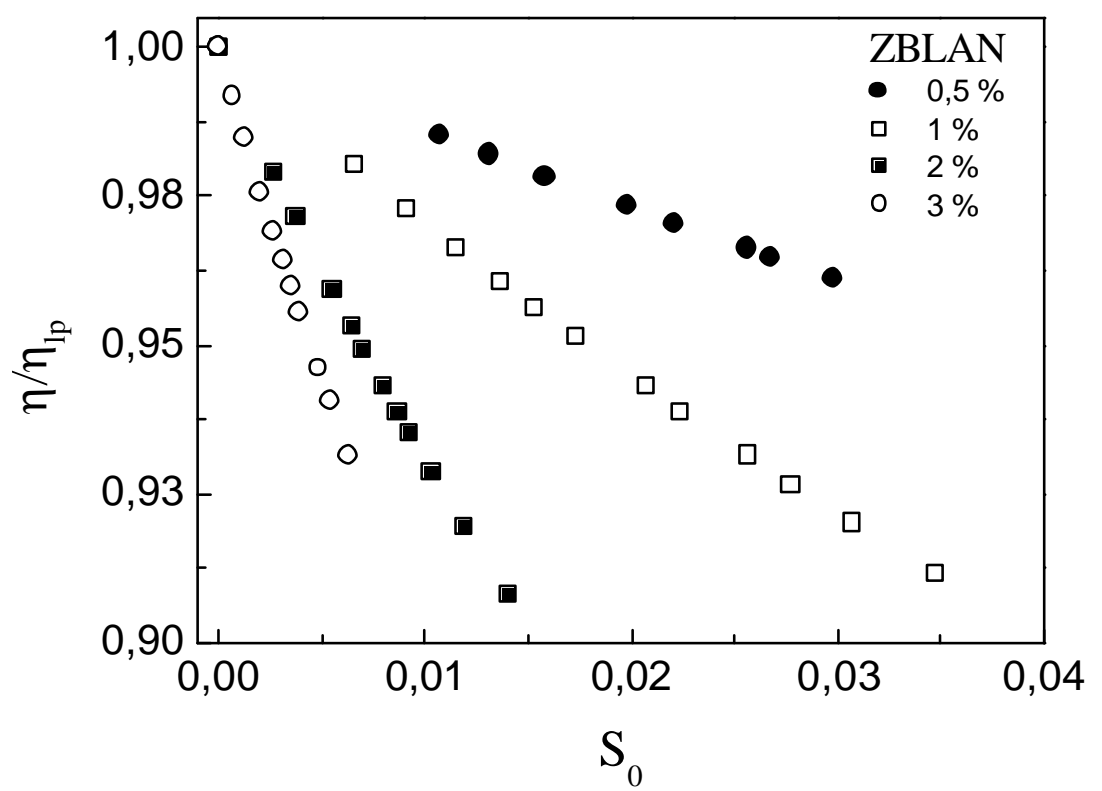

Figura 5.10 - Comportamento da eficiência quântica da fluorescência em função do parâmetro $S_{0}$. 
Após determinar o parâmetro $\beta$, o próximo passo foi determinar o parâmetro de relaxação cruzada $\left(?_{\text {rc }}\right)$ e isolar o parâmetro de "upconversion Auger" $\left(\gamma_{\text {up }}\right)$ dos resultados experimentais. Para isto, usamos $\quad ?_{\mathrm{rc}}=\mathrm{N}_{0} / \mathrm{t}_{0} \mathrm{~N}_{\mathrm{cq}}^{2} \quad$ (Eq. ((5.6)) com $\mathrm{t}_{0}=(527 \pm 11) 10^{-6} \mu \mathrm{s}$ e $\mathrm{N}_{\mathrm{cq}}=(5,3 \pm 0,2) \times 10^{20}$ ions $/ \mathrm{cm}^{3}$ obtidos no Capítulo 3 (vide Tabela 3.5), de modo que $?_{\mathrm{rc}}=\left[(6,8 \pm 0,6) \times 10^{-39} \mathrm{~cm}^{6} / \mathrm{s}\right] \mathrm{N}_{0}$. Usando a Eq. (5.5) e o valores de $\mathrm{N}_{0}, \mathrm{t}_{0}$ e $\mathrm{B}$ apresentados na Tabela 5.3, determinamos a dferença entre os parâmetros $?_{\text {up }}-?_{\text {rc }}$. Observamos uma dependência linear de $?_{\text {up }}-?_{\text {rc }}$ com a concentração de íons $\left(\mathrm{N}_{0} \sim 1-5 \times 10^{20}\right.$ íns $\left./ \mathrm{cm}^{3}\right)$ como mostra a Figura 5.11. Fazendo um ajuste linear dos dados encontramos que

$$
\left(?_{\text {up }}-?_{\text {rc }}\right)=(0,8 \pm 0,5) \times 10^{-17} \mathrm{~cm}^{3} / \mathrm{s}+\left[(2,1 \pm 0,1) \times 10^{-37} \mathrm{~cm}^{6} / \mathrm{s}\right] \mathrm{N}_{0}
$$

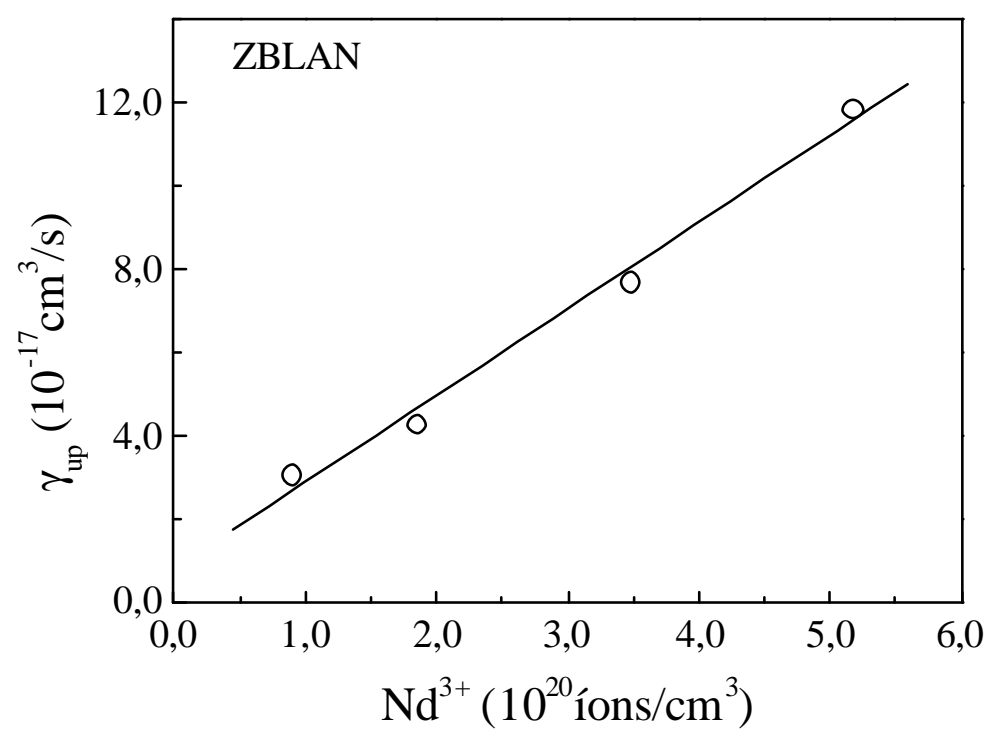

Figura 5.11 - Valores de ? ${ }_{u p}$ do fluorozirconato ( ZBLAN ) em função da concentração de $N d^{3+}$.

Usando o resultado $?_{\mathrm{rc}}=\left(6,8 \times 10^{-39} \mathrm{~cm}^{6} / \mathrm{s}\right) \mathrm{N}_{0}$ na Eq. (5.11), podemos observar que $?_{\text {up }}$ é aproximadamente duas ordens de magnitude maior do que $?_{\mathrm{rc}}$. 
Consequentemente, a aproximação $?_{\text {up }}-?_{\text {rc }} \sim ?_{\text {up }}$ pode ser feita, pois o efeito de $?_{\text {rc }}$ é muito menor que a incerteza experimental de ?

De acordo com Payne e colaboradores [50], o aumento linear de ? up com $\mathrm{N}_{0}$ caracteriza uma transferência de energia assistida por migração (conhecida como processo Burshstein ou "B") enquanto que a parte residual $(0,8 \pm 0,5) \times 10^{-17} \mathrm{~cm}^{3} / \mathrm{s}$ na Eq. (5.11) é devida a transferência estática (de Forster-Dexter ou F-D), onde os íons excitados permanecem fixos no espaço (não há migração). Consequentemente, a taxa total de "upconversion" é o resultado desses dois mecanismos, ou seja,

$$
\mathrm{W}_{\text {up }}=\mathrm{W}_{\mathrm{F}-\mathrm{D}}+\mathrm{W}_{\mathrm{B}}
$$

Podemos também escrever $\mathrm{W}_{\text {up }}=?_{\text {up }} \mathrm{N}_{\mathrm{ex}}$, onde $?_{\text {up }}=?_{\mathrm{F}-\mathrm{D}}+?_{\mathrm{B}}$ é $\mathrm{o}$ parâmetro de "upconversion" e $\mathrm{N}_{\mathrm{ex}}$ a população do estado excitado. Segundo Payne [50] $\mathrm{W}_{\mathrm{F}-\mathrm{D}} \propto \mathrm{N}_{\mathrm{ex}}$ e $\mathrm{W}_{\mathrm{B}} \propto \mathrm{N}_{\mathrm{ex}} \mathrm{N}_{0}, \log \mathrm{?_{ \textrm {F } - \mathrm { D } }}$ não depende da concentração e $?_{\mathrm{B}} \propto \mathrm{N}_{0}$. Usando um valor típico de $\mathrm{N}_{0}$ de vidros comerciais podemos ter uma idéia da grandeza $\gamma_{B}$. Por exemplo, usando o valor $\mathrm{N}_{0} \approx 1,8 \times 10^{20}$ ín $/ \mathrm{cm}^{3}$ (concentração típica usada em laser) na Eq. (5.11), obtemos $?_{\text {up }}=(0,8+3,8) \times 10^{-17} \mathrm{~cm}^{3} / \mathrm{s}$, ou seja, $?_{\mathrm{B}} \sim 4,8 ?_{\mathrm{F}-\mathrm{D}}$, o processo B é o dominante.

O valor de $?_{\text {up }}$ medido por Payne e colaboradores [50], por meio de medidas transientes para uma amostra ZBLAN com $2,5 \times 10^{20}$ íns $/ \mathrm{cm}^{3}$ de $\mathrm{Nd}^{3+}$, foi de $(4,6 \pm 1,4) \times 10^{-17} \mathrm{~cm}^{3} / \mathrm{s}$. Vale dizer que suas medidas foram realizadas próximo da potência de "threshold" da amostra, com um $\mathrm{N}_{\mathrm{ex}}$ ?t $=ß \mathrm{~S} \sim 0,63$. No entanto, nós medimos um vidro ZBLAN similar ao medido por Payne com um $N_{e x}$ muito menor, correspondendo a BS no intervalo de 0,015-0,03. Isto indica que a sensibilidade do 
nosso método é $\approx 20$ vezes maior. Dos valores apresentados na Figura 5.11, nós calculamos $?_{\text {up }} \sim 6,0 \times 10^{-17} \mathrm{~cm}^{3} / \mathrm{s}$ para uma mesma concentração de $\mathrm{Nd}^{3+}$ $\left(\mathrm{N}_{0}=2,5 \times 10^{20}\right.$ íns $\left./ \mathrm{cm}^{3}\right)$. A discrepância entre os resultados pode ser atribuída aos diferentes métodos utilizados na determinação do parâmetro "upconversion" e considerando todos os erros envolvidos, estes números podem ser considerados em bom acordo.

O mesmo procedimento foi utilizado para estudar os processos de transferência de energia no vidro fluoroindato (PGIZCa ), cujos resultados podem ser vistos na Figura 5.12, Figura 5.13, Figura 5.14 e Figura 5.15. Fazendo um ajuste linear nos dados experimentais apresentados na Figura 5.15, obtivemos $?_{\text {up }}=(0,8 \pm 0,5) \times 10^{-17} \mathrm{~cm}^{3} / \mathrm{s}+\left[(2,85 \pm 0,10) \times 10^{-37} \mathrm{~cm}^{6} / \mathrm{s}\right] \mathrm{N}_{0}$. Usando o valor do tempo de vida no limite de baixa concentração $t_{0}=(446 \pm 8) \times 10^{-6} \mu$ s e o valor de $\mathrm{N}_{\mathrm{cq}}=(4,8 \pm 0,2) \times 10^{20}$ íns $/ \mathrm{cm}^{3} \quad$ (vide valores apresentados na Tabela 3.5), determinamos $?_{\mathrm{rc}}=\left[(9,7 \pm 1) \times 10^{-39} \mathrm{~cm}^{6} / \mathrm{s}\right] \mathrm{N}_{0}$. Novamente podemos observar que $?_{\mathrm{rc}}$ é muito menor do que $?_{\text {up }}$.

Para o cristal YLF (com $\mathrm{N}_{0}=1,7 \times 10^{20}{ }_{10}$ ions $\left./ \mathrm{cm}^{3}\right)$ obtivemos $?_{\text {up }}=(1,9 \pm 0,6) \times 10^{-16} \mathrm{~cm}^{3} / \mathrm{s}$. Este valor está em bom acordo com o valor determinado por Guyot e colaboradores [101]. Eles determinaram através do método da fluorescência um $?_{\text {up }}=1,7 \times 10^{-16} \mathrm{~cm}^{3} / \mathrm{s}$ em uma amostra com $\mathrm{N}_{0}=2 \times 10^{20}$ íns $/ \mathrm{cm}^{3}$. 


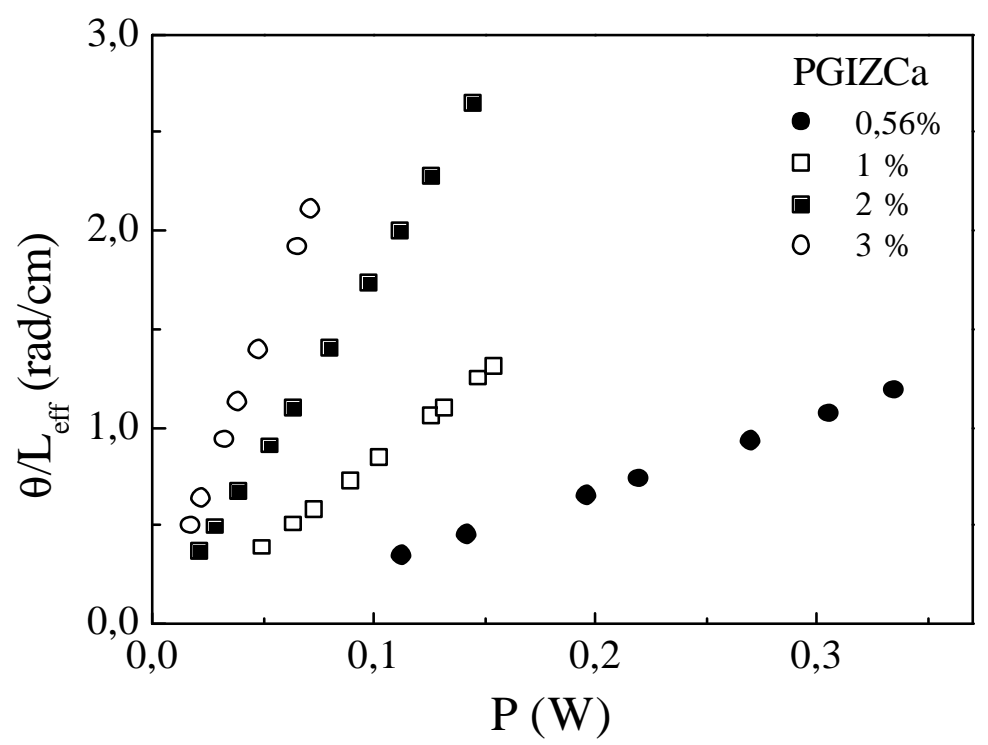

Figura 5.12 - Sinal de LT normalizado por $L_{\text {eff }}$ em função da potência do feixe de excitação em $?_{\mathrm{exc}}=797 \mathrm{~nm}$, no vidro fluoroindato $(P G I Z C a)$ dopado com $\mathrm{Nd}^{3+}$.

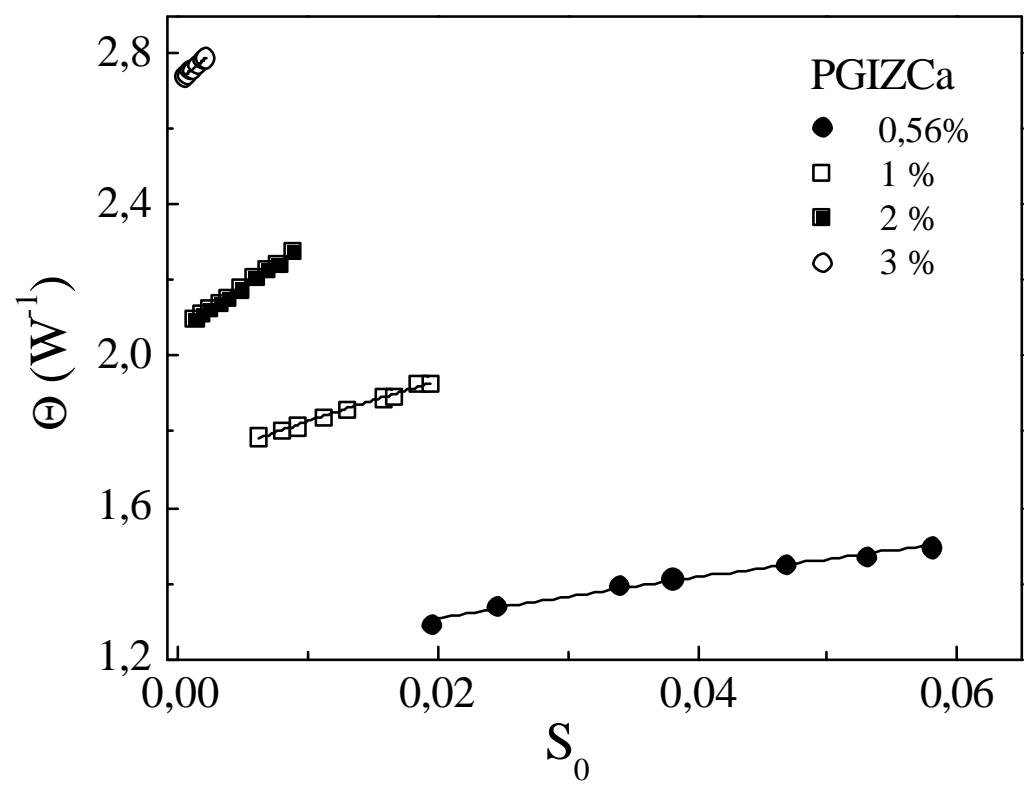

Figura 5.13 - $T$ em função do parâmetro de saturação $S_{0}$. Ajuste realizado com a Eq.(5.10). 


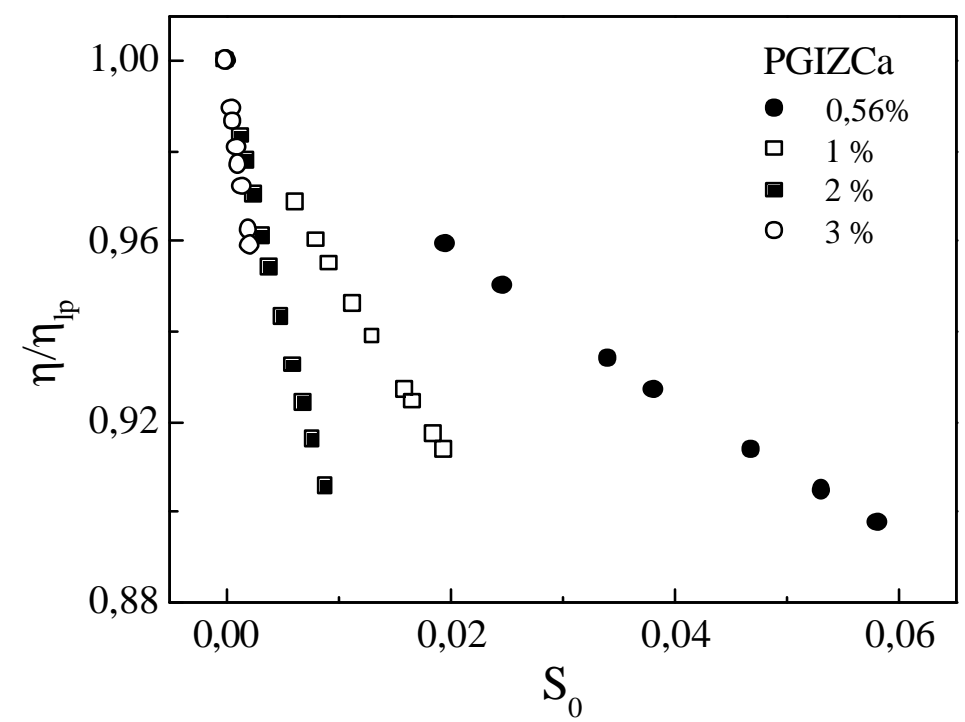

Figura 5.14 - Eficiência quântica da fluorescência em função do parâmetro $S_{0}$.

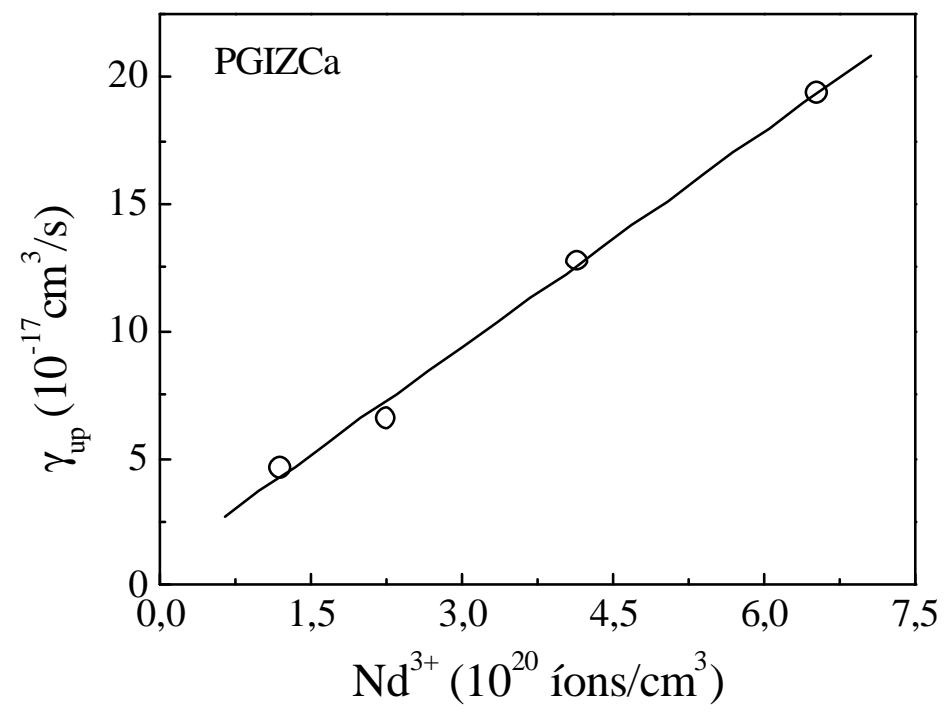

Figura 5.15 - Valores de ? em função $N_{0}$ para o PGIZCa.

\begin{tabular}{ccccccc}
\hline \hline Amostra & $\begin{array}{c}\mathrm{N}_{0} \\
\left(10^{20} \mathrm{~cm}^{-3}\right)\end{array}$ & $\begin{array}{c}\tau \\
(\mu s)\end{array}$ & $\begin{array}{c}\mathrm{L} \\
(\mathrm{cm})\end{array}$ & $\begin{array}{c}\mathrm{I}_{\mathrm{s}} \\
\left(\mathrm{KW} / \mathrm{cm}^{2}\right)\end{array}$ & $\mathrm{T}_{0}$ & $B$ \\
\hline \hline $\begin{array}{c}\text { Fluoroindato } \\
0,56 \%\end{array}$ & 1,2 & 421 & 0,24 & 29,6 & 1,2 & 2,3 \\
$1,0 \%$ & 2,3 & 367 & 0,24 & 34,0 & 1,7 & 5,4 \\
$2,0 \%$ & 4,2 & 248 & 0,24 & 50,3 & 2,1 & 13,1 \\
$3,0 \%$ & 6,5 & 164 & 0,22 & 76,0 & 2,7 & 20,7 \\
\hline$Y L F$ & 1,7 & 520 & 0,2 & 115 & 2,4 & 16,8 \\
\hline
\end{tabular}

Tabela 5.3 - Resultados do processo de transferência de energia, em função da concentração de $\mathrm{Nd}^{3+}$ no vidro fluoroindato ( PGIZCa) e no cristal YLF . 
No cristal de YAG, não foi possível determinar o parâmetro de transferencia de energia através das medidas de LT. Na Figura 5.16 temos o comportamento do sinal de LT normalizado pela absorção e pela espessura efetiva da amostra em função da potência de bombeio com excitação em $?_{\text {exc }}=514 \mathrm{~nm}$. Como mostramos para as outras amostras aqui estudadas, esperava-mos que houve-se um aumento no sinal de LT com a potência de bombeio. No entanto, observamos no YAG um efeito contrário (vide Figura 5.17). Houve um aumento no sinal até a potência de aproximadamente $200 \mathrm{~mW}$ que corresponde a um $\mathrm{S} \approx 0,03$. Um comportamento similar foi observado quando excitamos o cristal em $?_{\text {exc }}=869 \mathrm{~nm}$ (Figura 5.18 e Figura 5.19) e $?_{\mathrm{exc}}=808 \mathrm{~nm}$ (Figura 5.20 e Figura 5.21). Para $?_{\mathrm{exc}}=514 \mathrm{~nm}$ e $?_{\mathrm{exc}}=869 \mathrm{~nm}, \mathrm{~T}$ decai com $S>0,04$. Da Eq. (5.8), assumindo que não há contribuição do parâmetro $\beta$, temos que $\mathrm{N}_{\mathrm{ex}} \sim \mathrm{N}_{0} \mathrm{~S}$, usando o valor de $\mathrm{N}_{0}=1,03 \times 10^{20}$ íns $/ \mathrm{cm}^{3}$ (vide Tabela B 2), podemos estimar a população do estado excitado $\mathrm{N}_{\mathrm{ex}} \sim 3 \times 10^{18}$ íns $/ \mathrm{cm}^{3}$, que é cerca de 34 vezes menor do que a população total. Após esta potência o sinal sofreu uma forte queda, como pode ser visto na Figura 5.17 e Figura 5.19. Neste caso, nós ainda não temos uma explicação para o fenômeno que está ocorrendo. De modo que, para obter os parâmetros de LT, devemos considerar apenas os pontos onde o sinal é linear com a potência.

Mostraremos no Capítulo 6, que o parâmetro de transferência de energia também pode ser medido através da absorção saturada do estado excitado. A medida é feita utilizando a técnica conhecida como Z-scan (varredura-z). 


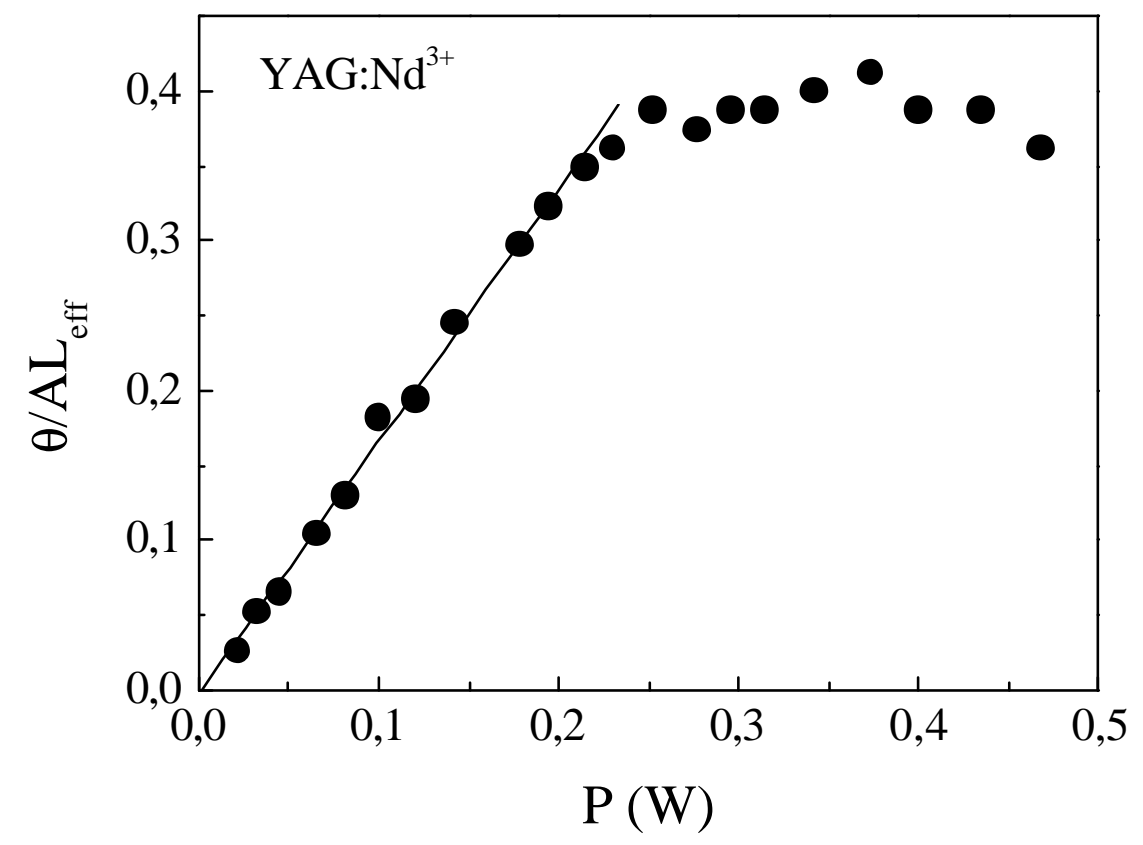

Figura 5.16 - Sinal de LT normalizado por $A L_{\text {eff }}$ em função da potência do feixe de excitação em $\lambda_{\text {exc }}=514 \mathrm{~nm}$.

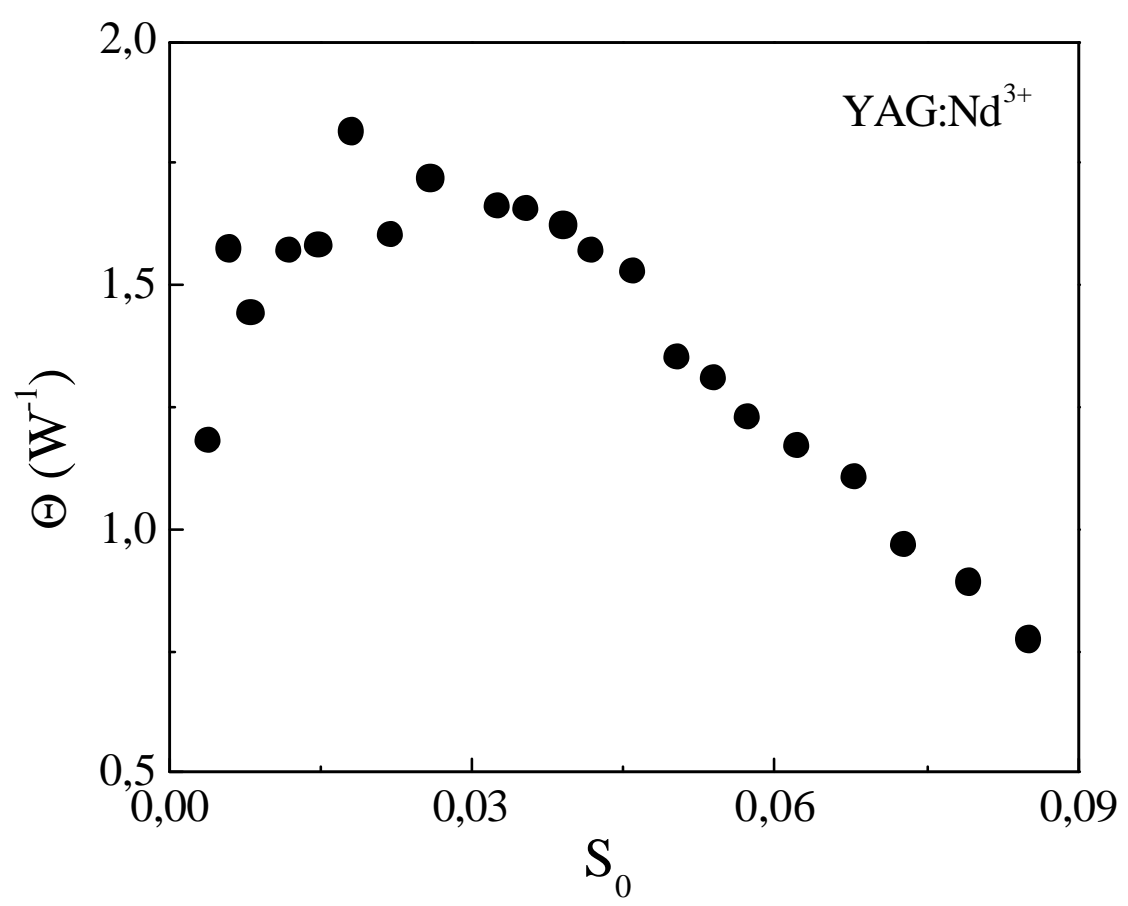

Figura 5.17 - T em função do parâmetro de saturação $S_{0}$, com $\lambda_{\text {exc }}=514 \mathrm{~nm}$. 


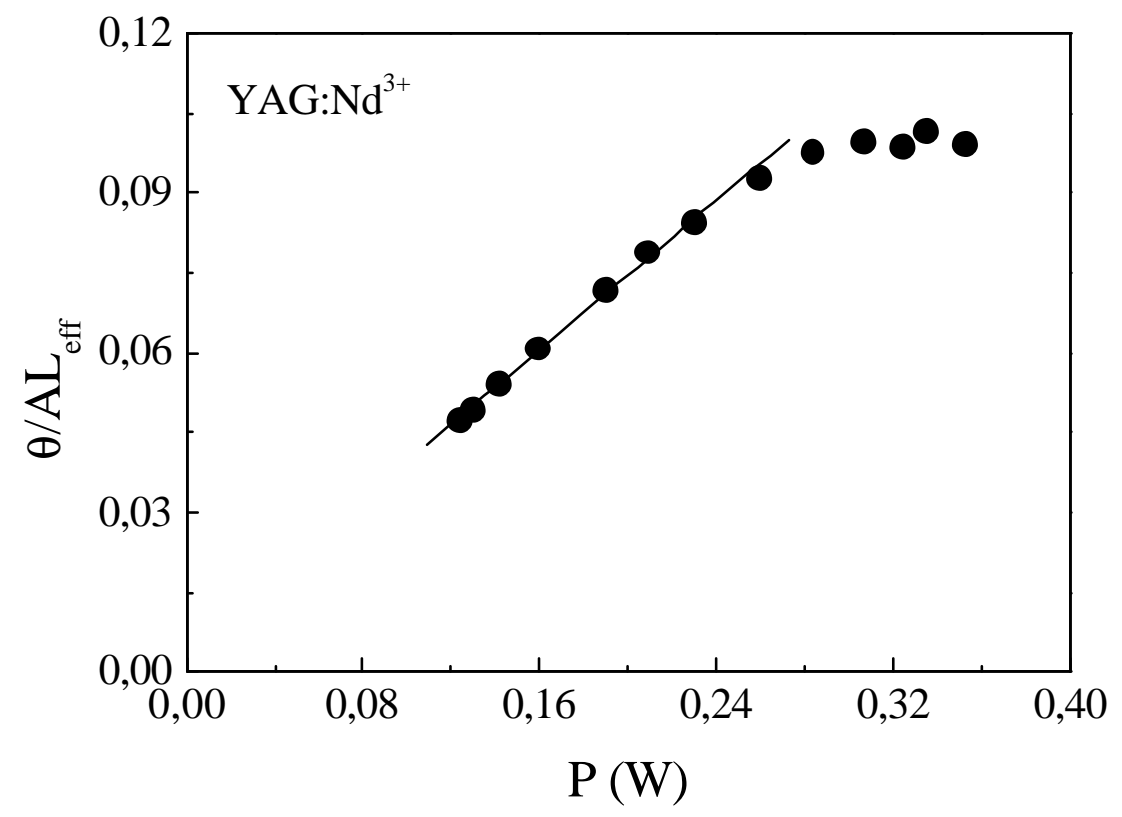

Figura 5.18 - Sinal de $L T$ normalizado por $A L_{\text {eff }}$ em função da potência do feixe de excitação em $\lambda_{\text {exc }}=869 \mathrm{~nm}$.

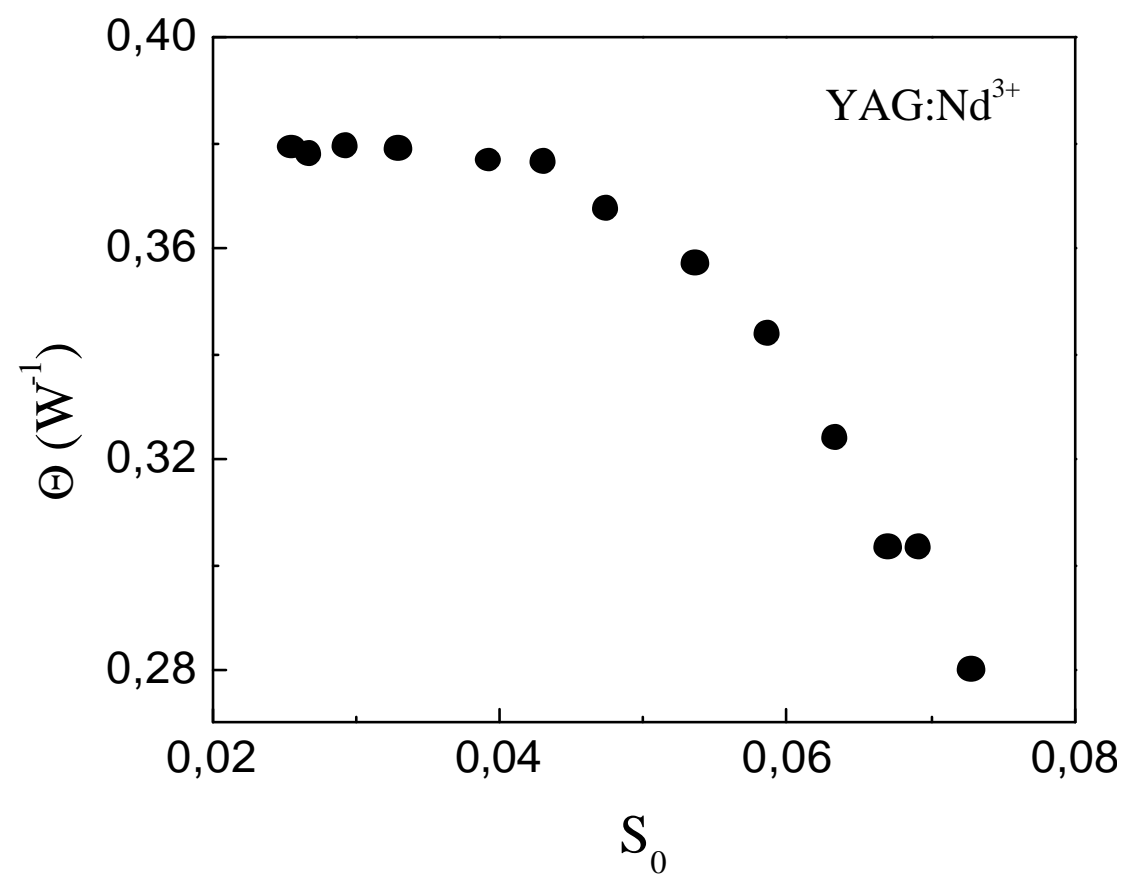

Figura 5.19 - T em função do parâmetro de saturação $S_{0}$, com $\lambda_{\text {exc }}=869 \mathrm{~nm}$. 


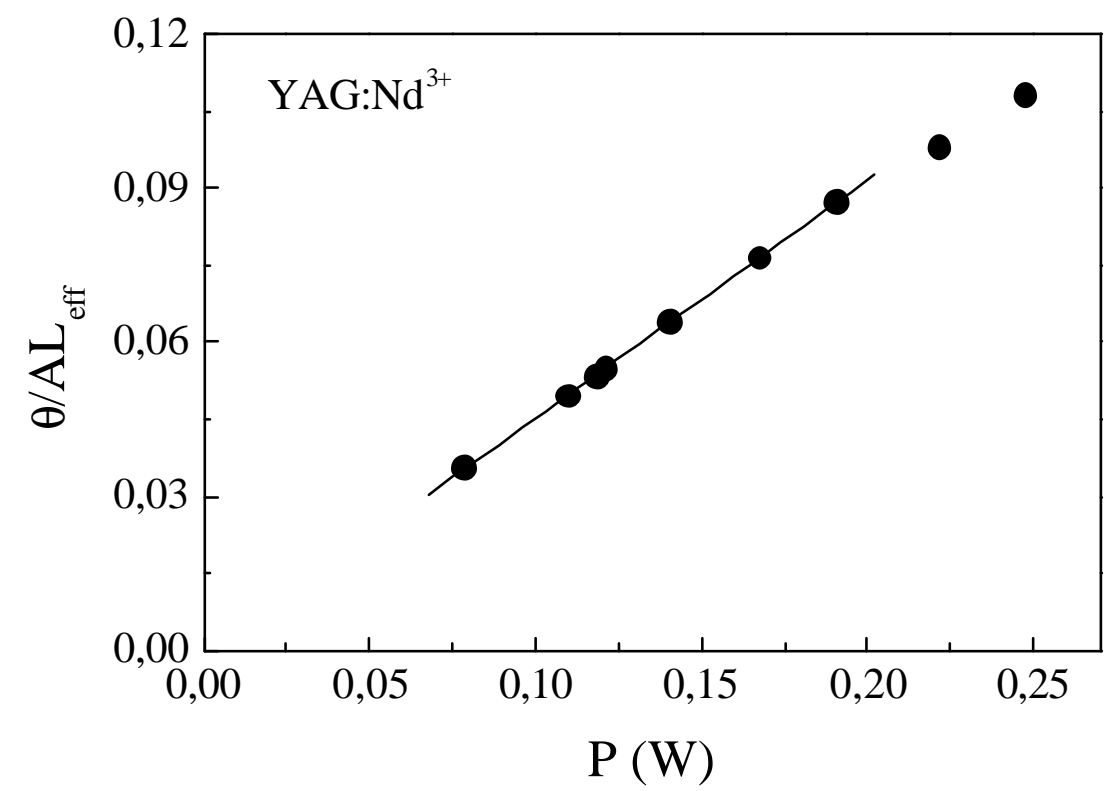

Figura 5.20 - Sinal de LT normalizado por $A L_{\text {eff }}$ em função da potência do feixe de excitação em $\lambda_{\text {exc }}=808 \mathrm{~nm}$.

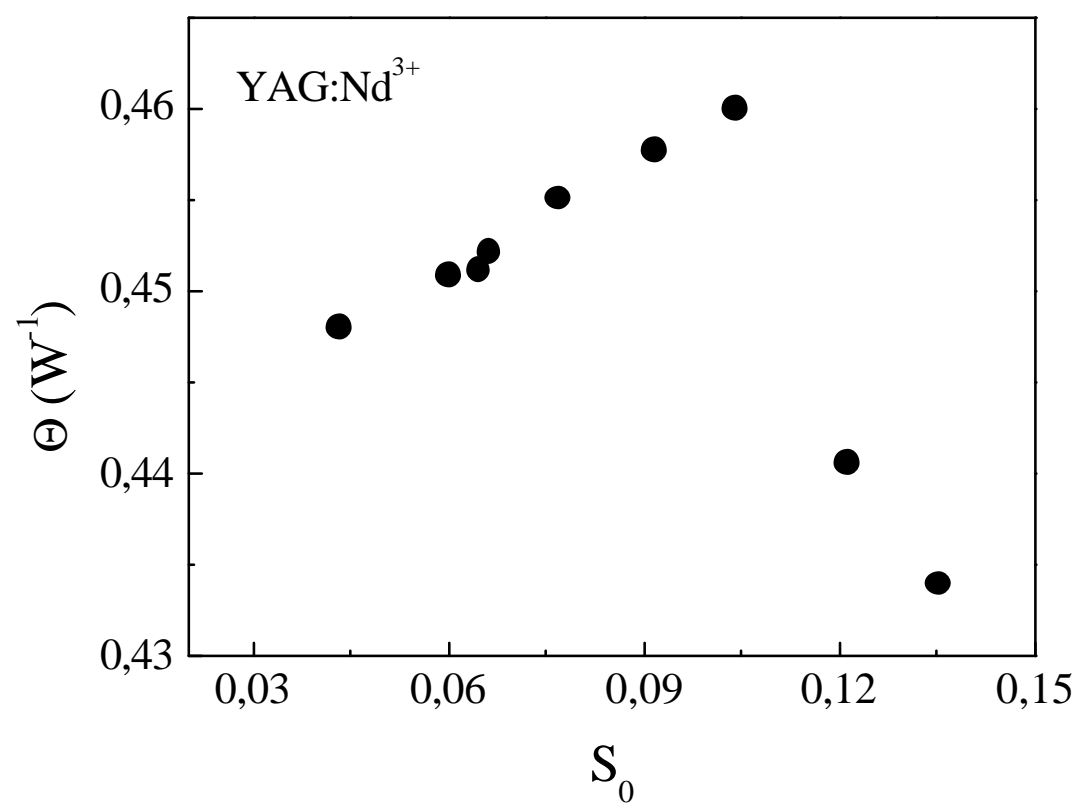

Figura 5.21 - T em função do parâmetro de saturação $S_{0}$, com $\lambda_{\text {exc }}=808 \mathrm{~nm}$. 


\section{Capítulo 6}

\section{Estudo dos efeitos de LP e LT através da técnica de Z-scan.}

Além das propriedades espectrocópicas lineares, as propriedades não-lineares são particularmente importantes no meio ativo para laser. E estas propriedades têm sido estudadas usando várias técnicas tais como interferométrica [1], mistura de onda [2-4], modulação espacial de fase [53], lente térmica [5, 6, 19] e Z-scan [5, 8-12, 22].

Neste capítulo iremos apresentar os resultados das medidas de Z-scan nos vidros fluoretos e nos cristais: YAG e YLF, dopados com $\mathrm{Nd}^{3+}$. Nós utilizamos a técnica de Z-scan para discriminar as não-linearidades eletrônica e térmica. Como apresentamos no Capítulo 1, a não-linearidade eletrônica aparece devido a diferença de polarizabilidade (? $\alpha$ ) entre o estado excitado e o fundamental, chamado efeito de Lente de População (LP). A não-linearidade térmica ocorre devido a conversão de energia absorvida em calor, chamado efeito de Lente Térmica (LT).

\subsection{Técnica de Z-scan.}

A técnica conhecida como Z-scan, foi desenvolvida em 1989 por SheikBahae e colaboradores [14] e é a mais popular para medidas de índice de refração não linear $\mathrm{n}_{2}$. Ela tem sido utilizada para estudos de diversos materiais assim como sólidos dopados com $\mathrm{Cr}^{3+}$ [102], vidros [9], semicondutores [103], materiais orgânicos [104], etc. A técnica de Z-scan permite determinar o sinal e a magnitude do índice de refração não-linear, o qual está relacionado com a susceptibilidade de 
terceira ordem $\left(?^{3}\right)$. A idéia básica da técnica, consiste em relacionar a variação da intensidade no centro do feixe no campo distante com a variação do índice de refração da amostra. Esta técnica se destaca pela simplicidade e alta sensibilidade, comparada com técnicas como: mistura de ondas e de interferometria não linear, que embora sensíveis, necessitam de um aparato experimental mais complexo.

A Figura 6.1 mostra o aparato experimental da técnica de varredura $z$. Um feixe laser que se propaga na direção $z$ é focalizado no plano $z=0$. A amostra é deslocada ao longo do eixo $z$, varrendo a região focal do feixe. A variável que se mede nesta varredura $z$, é a potência transmitida através de uma íris que é posicionada em frente ao detetor como função da posição $z$ da amostra. O resultado de uma medida é expresso em termos da transmitância normalizada, T(z), definida como a razão entre $\mathrm{P}(\mathrm{z})$ e a potência transmitida com a amostra longe do foco, onde a intensidade é suficientemente pequena para que se possa desprezar qualquer efeito não linear:

$$
\mathrm{T}(\mathrm{z})=\frac{\mathrm{P}(\mathrm{z})}{\mathrm{P}\left(|\mathrm{z}|>>\mathrm{z}_{\mathrm{c}}\right)}
$$

O seguinte exemplo qualitativo explica como a curva de Z-scan está relacionada com o índice de refração não-linear da amostra. Consideremos um material não-linear fino, isto é, com espessura menor do que o comprimento focal do feixe, tendo $\mathrm{n}_{2}>0$ e que pode ser considerado como uma lente fina de comprimento focal variável. Começando a varredura de uma distância longe do foco ( $z$ negativo) onde a intensidade do laser é fraca para que se possa observar efeitos não-lineares, a transmitância medida permanece relativamente constante $\mathrm{T}(\mathrm{z})=1$ (Figura 6.1). Como a amostra é movida através do foco, o aumento da intensidade conduz a um efeito de lente positiva que tende a colimar o feixe, conduzindo a uma expansão do 
feixe no plano da íris e um decréscimo na transmitância. Para $z$ positivo, a autofocalização aumenta a convergência do feixe, conduzindo a um estreitamento no plano da íris, o qual resulta em uma diminuição da transmitância medida [14]. Finalmente em $\quad \mathrm{z}>>\mathrm{z}_{\mathrm{c}}$ a transmitância novamente volta a tender ao valor 1. Esta análise mostra que a transmitância em função de $z$ possui um mínimo em $z<0$ e um máximo em $\mathrm{z}>0$ (vide Figura 6.1). De maneira análoga, para o caso em que o meio apresenta $\mathrm{n}_{2}<0$, podemos verificar que o comportamento será o oposto ao caso $\mathrm{n}_{2}>0$, ou seja, o meio se comporta como uma lente divergente e as posições do máximo e do mínimo de transmitância estarão invertidas em relação ao caso anterior $\left(\mathrm{n}_{2}>0\right)$.

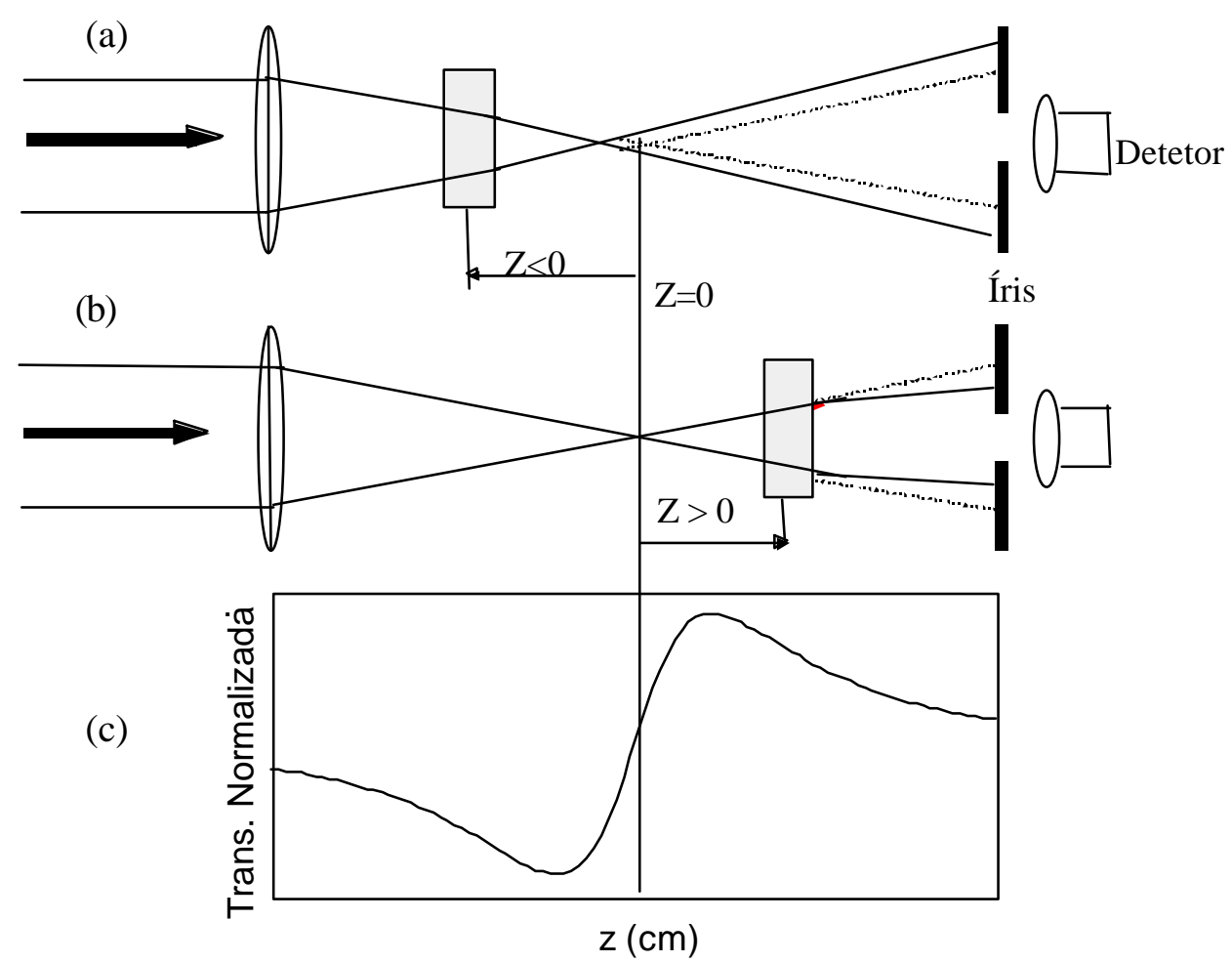

Figura 6.1 - Efeito de lente na técnica de Z-scan para o caso em que $n_{2}>0$. Em (a) temos o caso em que a amostra está situada antes do foco $(z<0)$ e o feixe aparece expandido na posição da íris. Em (b) a amostra se encontra após o foco $(z<0)$ e o feixe tende a ser colimado. Em (c) temos a curva característica de Z-scan, com $n_{2}>0$. A linha pontilhada representa o feixe quando o efeito não-linear é nulo $\left(n_{2}=0\right)$. 
O modelo teórico desenvolvido por Sheik-Bahae e colaboradores [105], fornece a expressão que descreve a curva obtida experimentalmente, isto é:

$$
\mathrm{T}\left(\mathrm{V}, ? \mathrm{~F}_{0}\right)=1+\frac{4 \mathrm{~V} ? \mathrm{~F}_{0}}{\left(9+\mathrm{V}^{2}\right)\left(1+\mathrm{V}^{2}\right)}
$$

sendo ? $\mathrm{F}_{0}=(2 \mathrm{p} / ?) \mathrm{L}_{\text {eff }} \mathrm{I}_{0} \mathrm{n}_{2}, \mathrm{I}_{0}=2 \mathrm{P} / \pi \mathrm{w}^{2}$ e $\mathrm{V}=\mathrm{z} / \mathrm{z}_{\mathrm{c}}$ (vide Eq. (2.4)). A expressão (6.2) tem a forma característica mostrada na Figura 6.1 com dois extremos, um máximo (posição de pico) e um mínimo (posição de vale) dados por $z= \pm 0,86 z_{c}$, de tal forma que a separação entre o pico e o vale seja:

$$
? \mathrm{Z}_{\mathrm{pv}} \sim 1,7 \mathrm{z}_{c}
$$

É importante observar que a medida de Zscan é feita no campo distante com uma abertura (íris) circular de raio $r_{a}$ (íris - Figura 6.1) posicionada em frente ao detetor. Assim sendo, é necessário que se leve em consideração o tamanho da abertura da fenda com a qual é realizado o experimento. Logo, a potência não é medida somente em $r=0$, o que corresponde a utilizar o raio da fenda $\left(r_{a}\right)$ infinitesimal quando comparado ao raio do feixe, w. Do ponto de vista experimental, é importante considerar o caso em que o raio da fenda $\left(r_{a}\right)$ e o tamanho do feixe sejam comparáveis, já que uma abertura muito pequena tende a ser muito sensível às flutuações do modo do laser e imperfeições da amostra. Logo, sendo w o valor da cintura do feixe no regime linear, a transmissão linear da fenda (sem a amostra) é dada por: $\mathrm{A}_{\mathrm{b}}=1-\exp \left[-2 \mathrm{r}_{\mathrm{a}}^{2} / \mathrm{w}^{2}\right]$. Baseando-se em resultados de simulações numéricas [105] verifica-se que quando o tamanho da abertura da fenda é levado em consideração, a expressão (6.2) precisa ser corrigida por um fator $\left(1-\mathrm{A}_{\mathrm{b}}\right)^{0,25}$. Desta maneira a variação de transmissão entre o pico e o vale fica dada por: 


$$
? \mathrm{~T}_{\mathrm{pv}} \sim 0,406\left(1-\mathrm{A}_{\mathrm{b}}\right)^{0,25} ? \mathrm{~F}_{0}
$$

No caso da fenda totalmente aberta $\left(\mathrm{A}_{\mathrm{b}}=100 \%\right)$ tem-se a seguinte relação:

$$
? \mathrm{~T}(\mathrm{~V})=\frac{4 \pi \mathrm{L}_{\text {eff }}}{?} \mathrm{n}_{2}=\frac{\mathrm{I}}{\left(1+\mathrm{V}^{2}\right)}
$$

As Eqs. (6.3), (6.4) e (6.5) são expressões fundamentais na determinação de $n_{2}$ de uma medida de Z-scan. Através dos dados experimentais de T(z) usa-se a variação de transmissão ? T para encontrar $\mathrm{n}_{2}$.

Entretanto, o modelo teórico desenvolvido por Sheik-Bahae e colaboradores só é válido somente para perfis de fase gaussiano [105]. Na ótica não-linear é muito importante o caso de não-linearidade saturável. Neste caso a dependência do índice de refração com a intensidade é dada por (Eq. (1.18)):

$$
\mathrm{n}=\mathrm{n}_{0}+\frac{\mathrm{n}_{2} \mathrm{I}}{1+\mathrm{I} / \mathrm{I}_{\mathrm{s}}}
$$

Uma vez que o laser tem um perfil de intensidade gaussiano I(r), pela Eq. (6.6) vemos que o perfil de índice de refração é gaussiano somente quando $\mathrm{I}<<\mathrm{I}_{\mathrm{s}}$. Como a teoria de Sheik-Bahae só é valida para perfil gaussiano, neste caso ela pode ser aplicada. Entretanto, a Figura 6.2 mostra que para $\mathrm{I}>\mathrm{I}_{\mathrm{s}}$ o perfil de índice de refração deixa de ser gaussiano e consequentemente a teoria de Sheik-Bahae deixa de ser aplicável.

Através de cálculos numéricos, Oliveira e colaboradores [11], obtiveram uma expressão empírica que relaciona a variação da transmitância normalizada ? $\mathrm{T}_{\mathrm{pv}}$ a $? \mathrm{~F}_{0}$ e $\mathrm{S}$ : 


$$
? \mathrm{~T}_{\mathrm{pv}} \approx 0.406\left(1-\mathrm{A}_{\mathrm{b}}\right)^{0.25(1+\mathrm{S})^{\left(1-\mathrm{A}_{\mathrm{b}}\right)}} \frac{? \mathrm{~F}_{0}}{(1+\mathrm{S})^{0.525}}
$$

onde $\mathrm{S}=\mathrm{I} / \mathrm{I}_{\mathrm{s}}$ é o parâmetro de saturação, ? $\mathrm{T}_{\mathrm{pv}}=\mathrm{T}_{\mathrm{p}}-\mathrm{T}_{\mathrm{v}}$ a diferença entre o pico e o vale de uma curva de Zscan. A equação acima pode ser considerada uma extensão da Eq. (6.4) para o caso de meios saturáveis. Foi obtido também uma expressão empírica para o caso do experimento com a fenda totalmente aberta $\left(\mathrm{A}_{\mathrm{b}}=1\right)$ :

$$
\mathrm{T}_{\mathrm{p}}-1 \approx \frac{2 \mathrm{p}}{?} \mathrm{n}_{2}^{\prime \prime} \mathrm{L}_{\text {eff }} \frac{\mathrm{I}_{0}}{(1+\mathrm{S})^{0.76}}
$$

sendo $\mathrm{T}_{\mathrm{p}}$ a transmissão normalizada em $\mathrm{z}=0$.

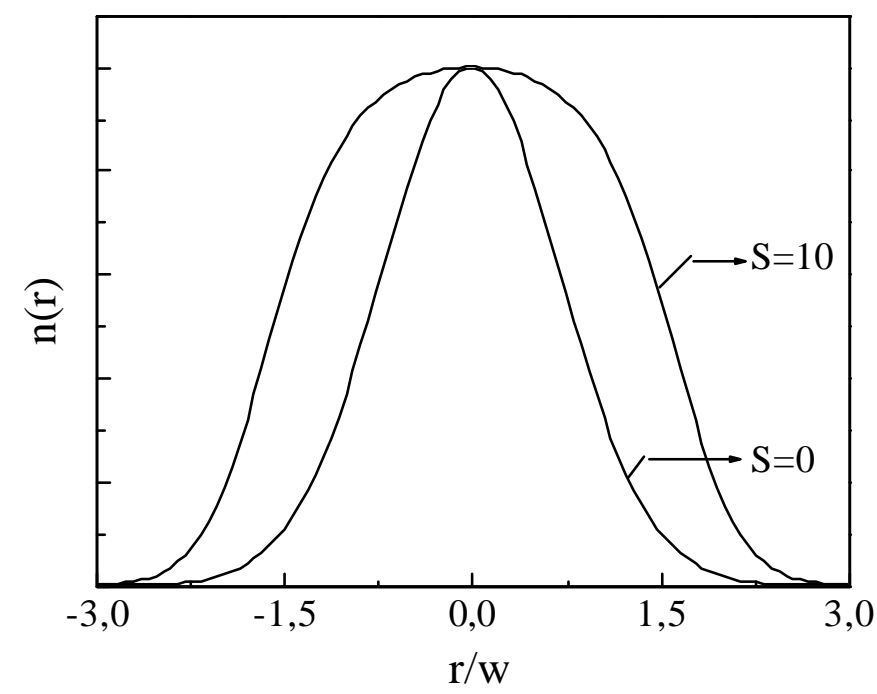

Figura 6.2 - Perfil do índice de refração $n(r)$ num meio não-linear saturável para $S=0$ e $S=10$.

\subsection{Montagem experimental.}

As medidas de Z-scan foram realizadas utilizando o aparato experimental mostrado na Figura 6.3. As lentes L-1 e L-2 foram dispostas da forma apresentada na figura para diminuir o tamanho do diâmetro do feixe de laser na posição do “chopper", pois com isto é possível diminuir o tempo que a pá do "chopper" leva 
para interromper o feixe. A amostra é deslocada ao longo da direção de propagação do feixe laser, próximo à posição focal da L-3 da figura. Um divisor de feixe Dv é utilizado para que se possa medir simultaneamente a parte real e a parte imaginária do índice de refração não-linear da amostra. A parte real devido à refração é medida pelo detetor D2 colocado atrás de uma íris (fenda) regulada para transmitir apenas a parte central do feixe (ela é ajustada para transmitir 50\%). A parte imaginária, devido à absorção, é captada pelo detetor D-1. Os sinais lidos pelos detetores são armazenados no sistema de aquisição [106].

Grande parte dos efeitos lineares provenientes do mau polimento ou não paralelismo das faces da amostra, podem ser eliminados normalizando uma medida realizada com alta intensidade por outra realizada com baixa intensidade. Imediatamente após a abertura da pá do "chopper", em $t_{i}=0$, os estados excitados metaestáveis com tempos de vida longos $(\sim$ ms) ainda não foram populados (no caso de Lente de População, LP) e/ou a amostra não recebeu energia suficiente para aumentar sua temperatura (no caso de Lente Térmica, LT). Quando o tempo evolui, os estados excitados são populados e/ou a amostra se aquece o suficiente para que os efeitos não lineares e/ou térmico comecem a se manifestar. Para $t_{f}>\mathrm{t}$ a população do estado excitado atinge seu estado estacionário e a transmitância apresenta contribuições lineares e não-lineares. O sistema de aquisição sincronizado com o sinal do "chopper", realiza uma medida de transmissão $\mathrm{T}\left(\mathrm{t}_{\mathrm{i}}\right)$ em $t=t_{i}$ e uma segunda $\mathrm{T}\left(\mathrm{t}_{\mathrm{f}}\right)$ em $t=t_{f}$ como mostra a Figura 6.4. Como imediatamente após a abertura do "chopper", em um tempo inicial $t=t_{i} \sim 0$, o laser não induz variação no índice de refração, de tal forma que a medida em $t_{f}$ pode ser normalizada pela medida em $t_{i}$. Este método permite também o estudo da evolução temporal do índice 
de refração o que é muito importante para distinguir os efeitos de LT e de LP. No Apêndice $\mathrm{C}$ é discutido em detalhes o fator de correção do tempo que deve ser levado em conta na análise dos resultados de uma varredura $\mathrm{z}$.

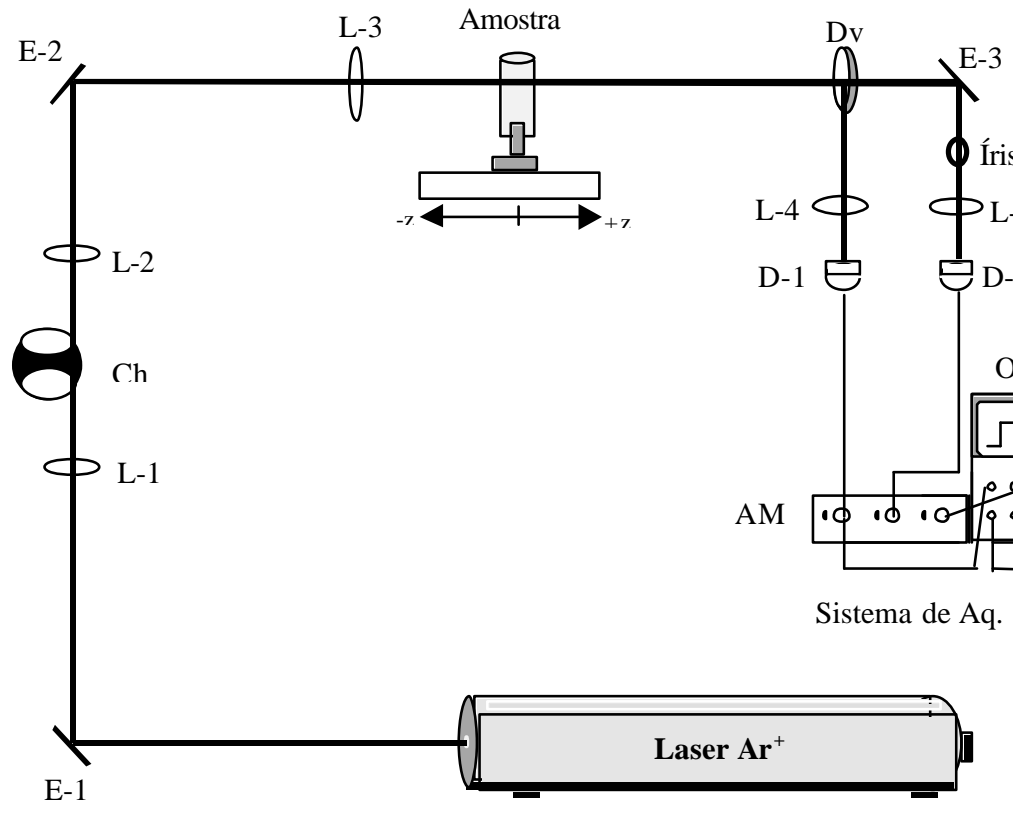

Figura 6.3 - Aparato experimental utilizado nas medidas de Z-scan. L representa lentes convergentes, E espelhos, D detetores, Dv divisor de feixe e Ch "chopper". Os sistema de aquisição é constituído por um microcomputador (M), um osciloscópio (OS) e um amplificador (AM).

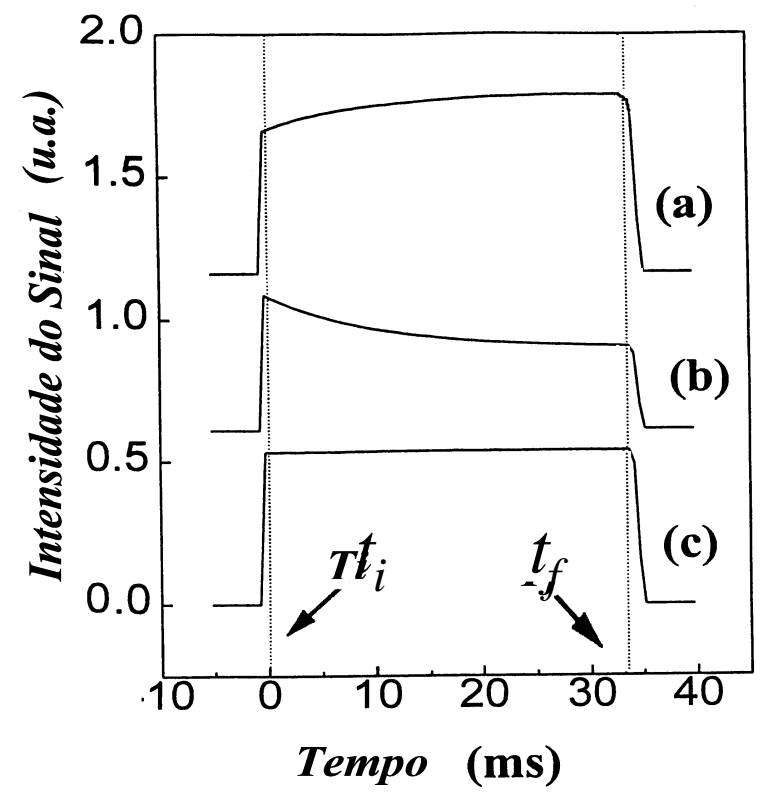

Figura 6.4 - Evolução temporal do sinal de Zscan da amostra de $\mathrm{GdAlO}_{3}: \mathrm{Cr}^{3+}$ $\left(n_{2}>0\right)$, colocada em (a) $z=0.85 z_{c}$, (b) $z=-0.85 z_{c}$ e (c) $z=7 z_{c}$ [11]. 


\subsection{Medidas nos cristais de YAG e YLF.}

As medidas de Z-scan, tanto no YAG quanto no YLF, foram realizadas com a frequência do chopper igual $820 \mathrm{~Hz}$. O tempo inicial escolhido foi $t_{i} \sim 20 \mu \mathrm{s}$ e o tempo final $t_{f} \sim 400 \mu s$. O propósito desta medida é escolher $\tau_{0}<t_{f}<t_{c}$ de maneira a termos em $t=t_{f}$, uma maior contribuição do sinal de Lente de População (LP) do que do sinal de Lente Térmica (LT).

Primeiramente, iremos apresentar os resultados obtidos no YAG. Nós estudamos duas amostras uma com $0,44 \%$ de $\mathrm{Nd}^{3+}$ e outra com 0,75\%. Mais detalhes sobres estas amostras podem ser vistos no Apêndice B As medidas foram realizadas excitando em $?=514 \mathrm{~nm}$ e $?=808 \mathrm{~nm}$.

A Figura 6.5 mostra os resultados de uma varredura $\mathrm{z}$ em $?=514 \mathrm{~nm}$ na amostra dopada com $0,44 \%$ de $\mathrm{Nd}^{3+}$. As medidas de Z-scan foram realizadas com a fenda em uma abertura de $\mathrm{A}_{\mathrm{b} 1}=50 \%$ e normalizada pela medida realizada com a fenda em abertura de $\mathrm{A}_{\mathrm{b} 2}=100 \%$, de modo a eliminar o efeito da absorção nãolinear. Podemos observar que a parte real do índice de refração não-linear $\mathrm{n}_{2}$ é positivo (fenda fechada), caracterizando um efeito de origem não-linear. Para este comprimento de onda $(?=514 \mathrm{~nm})$ estimamos uma intensidade de saturação $\mathrm{I}_{\mathrm{s}}=\mathrm{h} ? / \mathrm{st}=400 \mathrm{KW} / \mathrm{cm}^{2}$. A intensidade de bombeio que utilizamos foi de $42 \mathrm{KW} / \mathrm{cm}^{2}$, lembrando que $\mathrm{I}_{0}=2 \mathrm{P} / \mathrm{pw}_{0}^{2}$, onde no nosso experimento $\mathrm{P}=190 \mathrm{~mW}$ e $\mathrm{w}_{0}=17 \mu \mathrm{m}$. No experimento foi utlizado um lente com comprimento focal $f \sim 10 \mathrm{~cm}$. A partir da análise das medidas encontramos $\mathrm{n}_{2}{ }^{\prime}=1,4 \times 10^{-10} \mathrm{~cm}^{2} / \mathrm{W}$ e 
fazendo uso da Eq. (1.20), calculamos $? \mathrm{a}=8,0 \times 10^{-26} \mathrm{~cm}^{3}$. Para o YAG com $0,75 \%$ de $\mathrm{Nd}^{3+}$, encontramos $\mathrm{n}_{2}{ }^{\prime}=1,8 \times 10^{-10} \mathrm{~cm}^{2} / \mathrm{W}$ e $? \mathrm{a}=6,5 \times 10^{-26} \mathrm{~cm}^{3}$.

$\mathrm{O}$ resultado da medida feita com abertura da fenda em $\mathrm{A}_{\mathrm{b} 2}=100 \%$, Figura 6.5, nos possibilitou estimar, pela Eq. (1.21), apenas um limite superior para a parte imaginária $\mathrm{n}_{2} "<2,5 \times 10^{-12} \mathrm{~cm}^{2} / \mathrm{W}$.

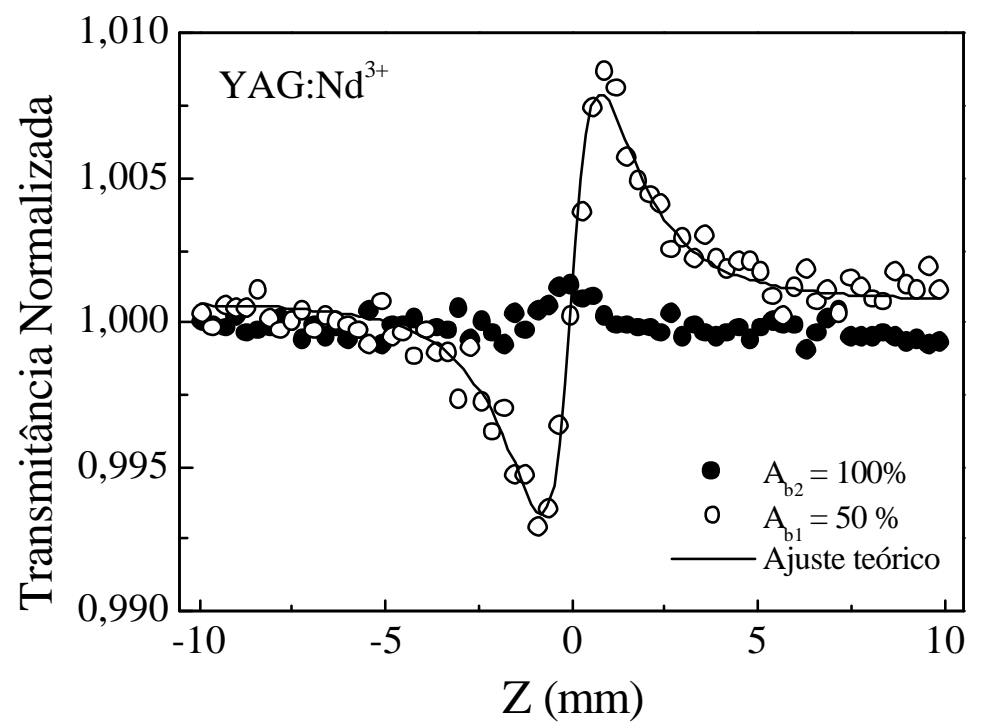

Figura 6.5 - Medida de Z-scan resolvido no tempo no cristal de YAG:Nd ${ }^{3+}$, com $0,44 \%$ de $\mathrm{Nd}^{3+}(L=1,4 \mathrm{~mm})$. Freqüência do chopper $(f=820 \mathrm{~Hz}), \lambda_{\text {exc }}=514 \mathrm{~nm}$ e potência $(P=190 \mathrm{~mW})$. Ajuste teórico feito com a expressão de Z-scan, Eq. (6.2) [107]. $A_{b 1}$ e $A_{b 2}$ representam a abertura da fenda.

$\mathrm{Na}$ Figura 6.6 temos o resultado da varredura $\mathrm{z}$ na amostra dopada com $0,44 \%$ de $\mathrm{Nd}^{3+}$, excitando em $808 \mathrm{~nm}$. Uma das vantagens de realizar as medidas excitando em 808nm, é que a energia de radiação incidente convertida em calor por fóton $\left(\sim 2970 \mathrm{~cm}^{-1}\right)$ é muito menor que no caso de excitação em $514 \mathrm{~nm}$, onde cada fóton converte $\sim 10600 \mathrm{~cm}^{-1}$ em calor, ou seja, em $808 \mathrm{~nm}$ temos $\varphi \approx 0,24$ e em 514nm $\varphi \approx 0,50$ (vide Eq. (2.6)). Sendo assim, o efeito de LT gerado pelas transições não radiativas podem ser minimizados. Neste comprimento de onda 
( $808 \mathrm{~nm})$ determinamos $\quad \mathrm{n}_{2}{ }^{\prime}=(2,7+1,5 \mathrm{i}) \times 10^{-9} \mathrm{~cm}^{2} / \mathrm{W}, \quad ? \mathrm{a}=6,2 \times 10^{-26} \mathrm{~cm}^{3} \quad$ e ? $\mathrm{s}=-5,8 \times 10^{-20} \mathrm{~cm}^{2}$. Para a amostra com $0,75 \%$ de $\mathrm{Nd}^{3+}$ obtivemos $\mathrm{n}_{2}{ }^{\prime}=(5,6+2,2 \mathrm{i}) \times 10^{-9} \mathrm{~cm}^{2} / \mathrm{W}$. Os resultados obtidos para as duas amostra de YAG estão apresentados na Tabela 6.1.
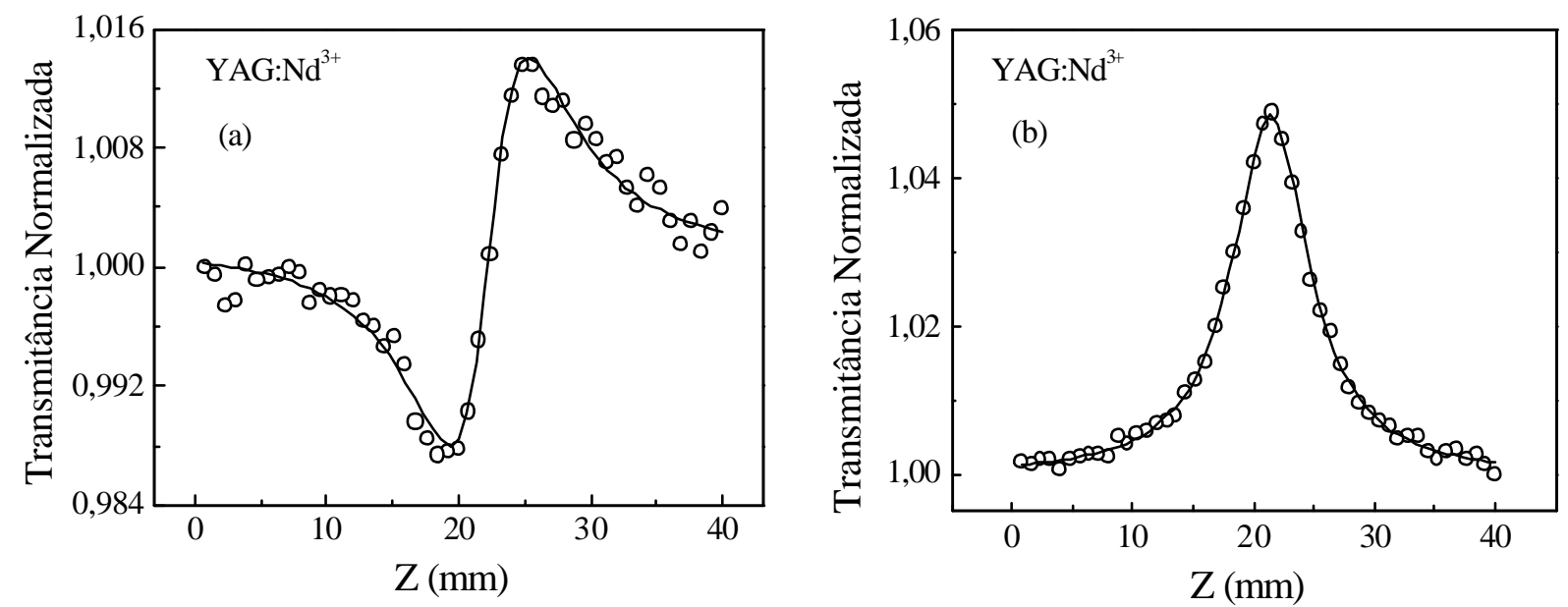

Figura 6.6 - Medida de Z-scan resolvido no tempo no cristal de YAG:Nd ${ }^{3+}(0,44 \%$ de $\mathrm{Nd}^{3+} \quad$ e $\left.L=0,14 \mathrm{~cm}\right)$, com $\lambda=808 \mathrm{~nm}, \quad P=102 \mathrm{~mW} \quad\left(I_{0}=7,2 \mathrm{KW} / \mathrm{cm}^{2}\right) \quad e$ $f=820 \mathrm{~Hz}$. (a) fenda fechada $A_{b 1}=50 \%$ (ajuste teórico realizado com a Eq. (6.2)); (b) fenda aberta $A_{b 2}=100 \%$ (ajuste realizado com a Eq. (6.5)) [107].

\begin{tabular}{|c|c|c|c|c|c|c|}
\hline YAG & $\begin{array}{c}\lambda \\
(\mathrm{nm})\end{array}$ & $\begin{array}{c}\sigma \\
\left(10^{-20} \mathrm{~cm}^{2}\right)\end{array}$ & $\begin{array}{c}\mathrm{I}_{\mathrm{s}} \\
\left(\mathrm{KW} / \mathrm{cm}^{2}\right)\end{array}$ & $\begin{array}{c}\mathrm{N}_{0} \\
\left(10^{19} \mathrm{~cm}^{-3}\right)\end{array}$ & $\begin{array}{c}\Delta \alpha \\
\left(10^{-26} \mathrm{~cm}^{3}\right)\end{array}$ & $\begin{array}{c}\Delta \sigma \\
\left(10^{-20} \mathrm{~cm}^{2}\right)\end{array}$ \\
\hline \multirow[t]{2}{*}{$0,44 \%$} & 514 & 0,42 & 400 & 6,1 & 8,0 & \\
\hline & 808 & & 15,3 & & 6,2 & $-5,8$ \\
\hline \multirow[t]{2}{*}{$0,75 \%$} & 514 & $7,0^{\text {(a) }}$ & & 10,3 & 6,5 & \\
\hline & 808 & & & & 6,2 & $-5,1$ \\
\hline
\end{tabular}

Tabela 6.1 - Valores dos parâmetros obtidos no YAG. (a) valor obtido da Ref. [108]. O valor de $I_{s}$ foi obtido através da relação $I_{s}=h$ ?/?st. 
Comparando os resultados das duas amostras estudadas, podemos observar que em 514nm, a amostra mais dopada apresenta ?a 1,2 vezes menor que a amostra menos dopada. Esta diferença pode ser entendida pelo provável erro na determinação do coeficiente de absorção em 514nm na amostra com menor concentração. A Figura B 2 do Apêndice B mostra o espectro de absorção da amostra com $0,44 \%$, e nele podemos observar que existe uma banda larga de absorção, na região entre $400-700 \mathrm{~nm}$, provavelmente devido a algum tipo de impureza. Isto dificulta uma obtenção precisa do valor do coeficiente de absorção interferindo no resultado final de ? a

Os resultados de ?a, tanto em 514nm quanto em $808 \mathrm{~nm}$, estão em razoável acordo o resultado obtido por Powell [109], ? a $=4,9 \times 10^{-26} \mathrm{~cm}^{3} \mathrm{em} 514 \mathrm{~nm}$ (vide Figura 1.4 do Capítulo 1), através de mistura de quatro ondas. Há uma diferença de aproximadamente $30 \%$ entre os dois resultados. Entretanto, acreditamos que nosso resultado seja mais confiável devido a técnica experimental utilizada e ao fato de que fizemos medidas em $514 \mathrm{~nm}$ e $808 \mathrm{~nm}$ (não é esperado que $\Delta \alpha$ varie muito com $\lambda$ nesta região).

Uma outra maneira de se determinar $\mathrm{n}_{2}{ }^{\prime}$ e $\mathrm{I}_{\mathrm{s}}$, é analisar o comportamento da variação do snal $\left(\right.$ ? $\left.\mathrm{T}_{\mathrm{pv}}\right)$ da fenda fechada em função da intensidade de bombeio, $\mathrm{I}_{0}$, como pode ser visto na Figura 6.7. Fazendo o ajuste dos dados experimentais com a Eq. (6.7), obtivemos $\quad \mathrm{n}_{2}{ }^{\prime}=(5,7 \pm 0,1) \times 10^{-9} \mathrm{~cm}^{2} / \mathrm{W} \quad$ e $\quad I_{\mathrm{s}}=(17,6 \pm 1,1) \mathrm{KW} / \mathrm{cm}^{2}$.

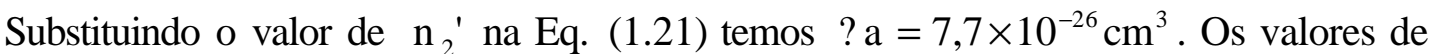
$I_{s}$ e ? a obtidos pelo ajuste da Figura 6.7, é cerca de $15 \%$ e $25 \%$, respectivamente, maior do que os valores apresentados na Tabela 6.1. Esta diferença pode ser devida ao fato de que, na equação de ajuste (Eq. (6.7)), não é considerado o efeito da 
transferência de energia (discutido no Capítulo 5), mas apenas o efeito de saturação S.

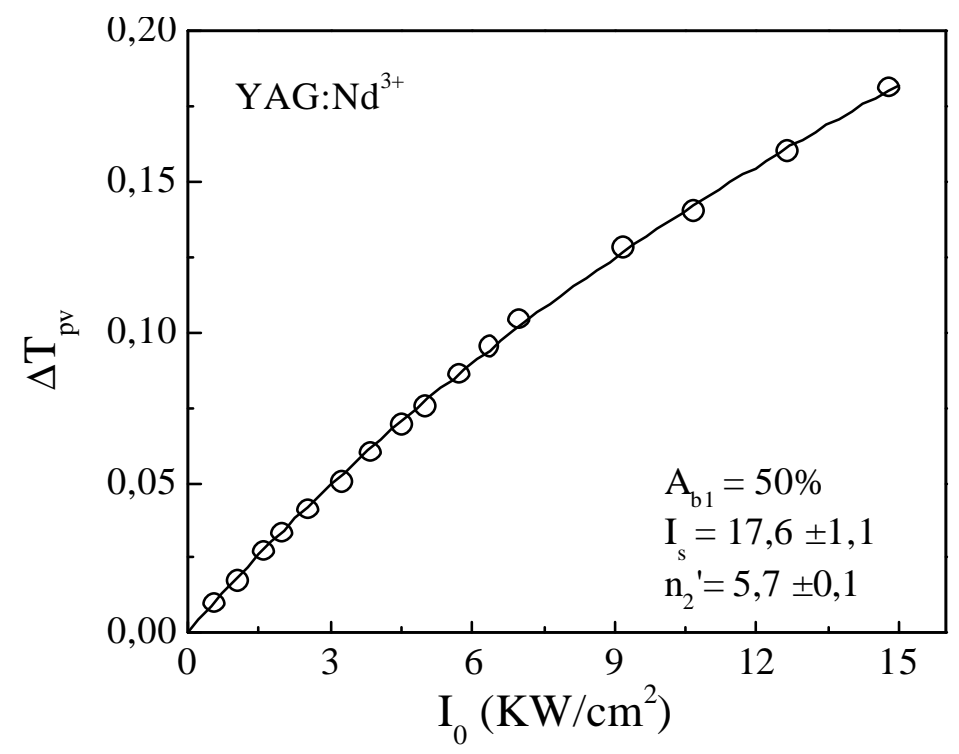

Figura 6.7 - Variação de ? $T_{\mathrm{pv}}\left(Y A G\right.$ com $0,75 \%$ de $\left.\mathrm{Nd}^{3+}\right)$ em função da intensidade, $I_{0}$, com $A_{b 1}=50 \%$ e $\lambda=808 \mathrm{~nm}$.

Veremos agora que no caso da fenda aberta encontramos uma expressão que considera o efeito da transferência de energia, de modo que podemos determinar, através do sinal da amplitude da fenda aberta, o parâmetro $\beta$ (Eq. (5.5)) não está incluído na Eq. (6.8).

Antes vamos escrever a expressão para a transmitância normalizada, ?T(r) , para o caso onde não é considerado os processos de transferência de energia. Para isto vamos considerar que o coeficiente de absorção seja dado por:

$$
\mathrm{A}=\mathrm{N}_{\mathrm{g}} \sigma_{\mathrm{g}}+\mathrm{N}_{\mathrm{ex}} \sigma_{\mathrm{ex}}
$$

como $\mathrm{N}_{\mathrm{g}}=\mathrm{N}_{0}-\mathrm{N}_{\mathrm{ex}}$, temos que $\mathrm{A}=\mathrm{A}_{0}+\mathrm{N}_{\mathrm{ex}}$ ? $\mathrm{s}$, onde ? $\mathrm{s}$ é a diferença da seção de choque de absorção entre os estados excitado $\sigma_{e x}$ e fundamental $\mathrm{s}_{\mathrm{g}}$. Usando a Lei de Lambert-Beer $\mathrm{T}=\mathrm{e}^{-\mathrm{AL}}$ podemos escrever 


$$
\mathrm{T}=\mathrm{e}^{-\mathrm{A}_{0} \mathrm{~L}}\left[1-\mathrm{N}_{\mathrm{ex}} \Delta \sigma \mathrm{L}\right]
$$

$\mathrm{Ou}$

$$
\mathrm{T}=\mathrm{T}_{0}(1-? \mathrm{~T})
$$

com

$$
\mathrm{T}_{0}=\mathrm{e}^{-\mathrm{A}_{0} \mathrm{~L}}
$$

e

$$
? \mathrm{~T}=\mathrm{N}_{\mathrm{ex}} \Delta \sigma \mathrm{L}
$$

Da Eq. (5.1) temos que:

$$
\mathrm{N}_{\mathrm{ex}} \sim \mathrm{N}_{0} \frac{\mathrm{S}}{1+\mathrm{S}}
$$

sendo $S=I(r) / I_{s}$ o parâmetro de saturação. Substituindo a Eq. (6.14) na Eq. (6.13) temos:

$$
? \mathrm{~T}(\mathrm{r})=? \mathrm{sLN}_{0} \frac{\mathrm{S}}{1+\mathrm{S}}
$$

Como em nosso modelo teórico assumimos que o feixe laser tem um perfil gaussiano de intensidade de modo TEM $_{00}$ (que na posição de foco é dado pela Eq. (2.6)), a Eq. (6.15) deve ser integrada em toda a área da amostra. Como estamos tratando do caso da fenda totalmente aberta, isto é, com $r>>w$, a integral deve ser feita de 0 a $\infty$, de modo que a solução para a Eq. (6.15) é dada por:

$$
? \mathrm{~T}=\frac{\mathrm{S}_{0}-\ln \left[1+\mathrm{S}_{0}\right]}{\mathrm{S}_{0}} ? \mathrm{~s} \mathrm{~N}_{0} \mathrm{~L}
$$

onde $\mathrm{S}_{0}=\mathrm{I}_{0} / \mathrm{I}_{\mathrm{s}}$.

Considerando agora os processos de transferência de energia, devemos escrever a expressão para a transmitância normalizada ?T(r), utilizando $\mathrm{N}_{\mathrm{ex}}$ (Eq. (5.8)) dado por: 


$$
\mathrm{N}_{\mathrm{ex}}(\mathrm{S})=\frac{-(\mathrm{S}+1) \pm \sqrt{(\mathrm{S}+1)^{2}+4 \mathrm{~S} \beta}}{2 \beta} \mathrm{N}_{\mathrm{o}}
$$

de modo que

$$
? \mathrm{~T}(\mathrm{r})=\left[\frac{-(1+\mathrm{S})+\sqrt{(1+\mathrm{S})^{2}+4 \beta \mathrm{S}}}{2 \beta}\right] ? \mathrm{sLN}_{0}
$$

sendo $\beta=\mathrm{t}$ ? ${ }_{\text {up }} \mathrm{N}_{0}$ (Eq. (5.5)). Porém, esta equação não tem uma solução analítica, tal que é possível obter apenas uma solução numérica. Na Figura 6.8 temos simulações teóricas comparando o resultado da Eq. (6.18), integrando e sem integrar. Podemos notar que para o parâmetro de saturação $S_{0}$ até aproximadamente 1 , existe uma diferença de aproximadamente $8 \%$ entre as curvas. De modo que em uma primeira aproximação, a Eq. (6.18) pode ser utilizada para ajustar os dados experimentais, pois em nossas medidas os máximos valores de $S_{0}$ usados foram até $S_{0} \sim 0,5$. A Figura 6.8 também mostra a simulação de ? T utilizando a Eq. (6.16), caso onde $\beta=0$.

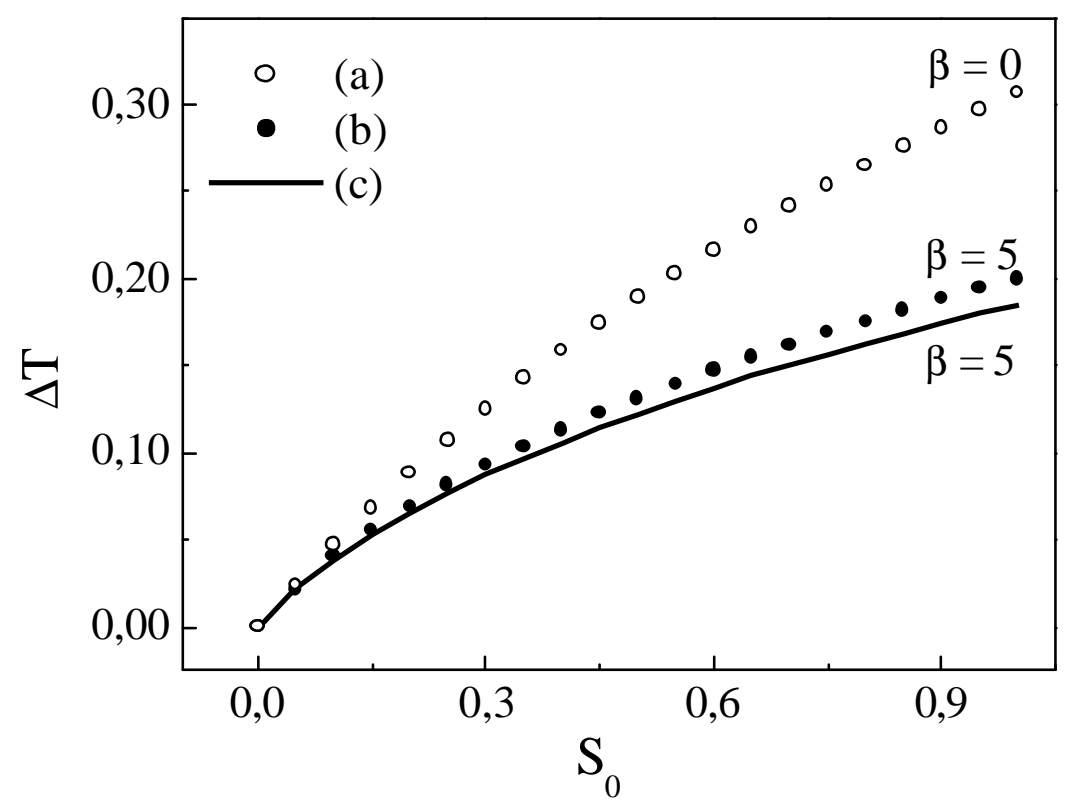

Figura 6.8 - Simulações teóricas de ? T versus $S_{0}$. (a) $\beta=0$ com a Eq. (6.16), (b) $\beta=5$ com a Eq. (6.18) integrada e (c) $\beta=5$ com a Eq. (6.18) sem integrar. 
A Figura 6.9 mostra a variação da amplitude $\left(T_{p}-1\right)$ do sinal da fenda aberta em função do parâmetro de saturação $\mathrm{S}_{0}$. Usando a Eq. (6.18), ajustamos os dados experimentais obtendo $\quad \beta=(5,7 \pm 1,1)$ e $? \mathrm{~s}=-(4,5 \pm 2) \times 10^{-20} \mathrm{~cm}^{2}$. Usando a Eq.(5.5) determinamos $?_{\text {up }}=(2,4 \pm 0,5) \times 10^{-16} \mathrm{~cm}^{3} / \mathrm{s}$. O valor de $?_{\text {up }}$ está próximo do valor $?_{\text {up }}=(2,8 \pm 1,0) \times 10^{-16} \mathrm{~cm}^{3} / \mathrm{s}$ reportado por Guyot e colaboradores [101] enquanto Guy e colaboradores obtiveram $?_{\text {up }}=0,5 \times 10^{-16} \mathrm{~cm}^{3} / \mathrm{s}$ [49]. O valor ?s obtido deste ajuste, é cerca de $15 \%$ menor do que o valor apresentado na Tabela 6.1. Como os valores apresentados na Tabela 6.1 foram obtidos com medidas realizadas com baixa potência, onde o efeito de "upconversion" não contribui, e considerando os erros experimentais envolvidos, podemos considerar que os resultados estão em bom acordo. Mostramos também na Figura 6.9 a curva teórica considerando o caso $\beta=0$.

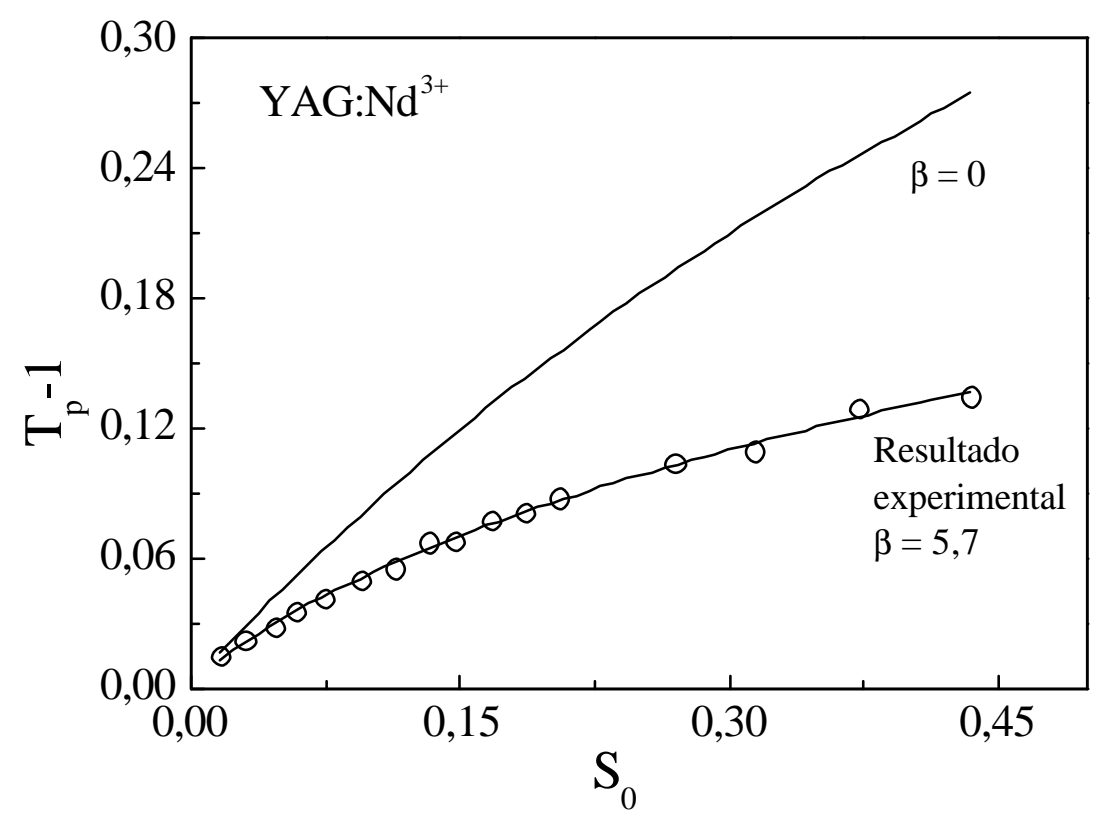

Figura 6.9 - Variação da amplitude do sinal da fenda aberta em função do parâmetro de saturação $S_{0}$. YAG com $0,75 \%$ de $\mathrm{Nd}^{3+}$. Ajuste realizado com a Eq. (6.18) usando $I_{s}=17,6 \mathrm{KW} / \mathrm{cm}^{2}$. Do ajuste obtivemos $\beta=5,7 \pm 1,1$ e $? \mathrm{~s}=-(4,5 \pm 2) \times 10^{-20} \mathrm{~cm}^{2}$. 
Estudamos também a evolução temporal do sinal de Zscan, tal como mostra a Figura 6.10. Este estudo foi primeiramente realizado por Pilla [12], estudando o comportamento transiente do sinal de Z-scan no regime de saturação em cristais dopados com $\mathrm{Cr}^{3+}$. Foi observado uma resposta exponencial proporcional $\left(1-\mathrm{e}^{-\mathrm{t} / \mathrm{t}}\right)$ com tempo de resposta, $\tau$, dependendo da intensidade como $\mathrm{t}^{-1}=\mathrm{t}_{0}^{-1}\left(1+0,85 \mathrm{I}_{\mathrm{p}} / \mathrm{I}_{\mathrm{s}}\right)$ [110]. O fator 0,85 foi encontrado através de cálculos numéricos da integral de difração de Kirchhoff [110]. A dependência linear de $\mathrm{t}^{-1}$ com $\mathrm{I} / \mathrm{I}_{\mathrm{s}}$ é esperada a partir das equações de taxa [106]. Deve-se notar, que no experimento I(r) tem um perfil gaussiano sendo $I_{p}$ a intensidade axial no ponto de pico $\left(z=0,86 z_{c}\right.$ e $\left.\mathrm{I}_{\mathrm{p}}=0,575 \mathrm{I}_{0}\right)$.

A Figura 6.10 (a) mostra um resultado de transiente típico com intensidade baixa $\left(\mathrm{I}_{\mathrm{p}}=2,7 \mathrm{KWcm}^{-2} \approx 0,17 \mathrm{I}_{\mathrm{s}}\right)$. O comportamento do sinal obtido é similar ao comportamento $\mathrm{N}_{\mathrm{ex}}(\mathrm{t})$ dado pela Eq. (1.7), como é esperado para uma absorção não linear que é devido ao efeito de LP. A medida foi realizada posicionando a amostra na posição de pico (posição de transmissão máxima) da Figura 6.6. As curvas $\mathrm{A}_{\mathrm{b} 1}=50 \%$ e $\mathrm{A}_{\mathrm{b} 2}=100 \%$ foram ajustados com um decaimento exponencial onde encontramos $t_{0}=(230 \pm 8) \mu$ s e $t_{0}=(250 \pm 10) \mu s$, respectivamente. Como esperado, para baixa potência, este resultado é igual ao tempo de vida $t=230 \mu$ s [51]. A Figura 6.10 (b) mostra a dependência linear de $\mathrm{t}^{-1}$ versus $\mathrm{I}_{\mathrm{p}}$. Ajustando com $\mathrm{t}^{-1}=\mathrm{t}_{0}^{-1}\left(1+0,85 \mathrm{I}_{\mathrm{p}} / \mathrm{I}_{\mathrm{s}}\right)$ obtivemos $\mathrm{t}_{0}$ e $\mathrm{I}_{\mathrm{s}} \quad$ (vide Tabela 6.2) 

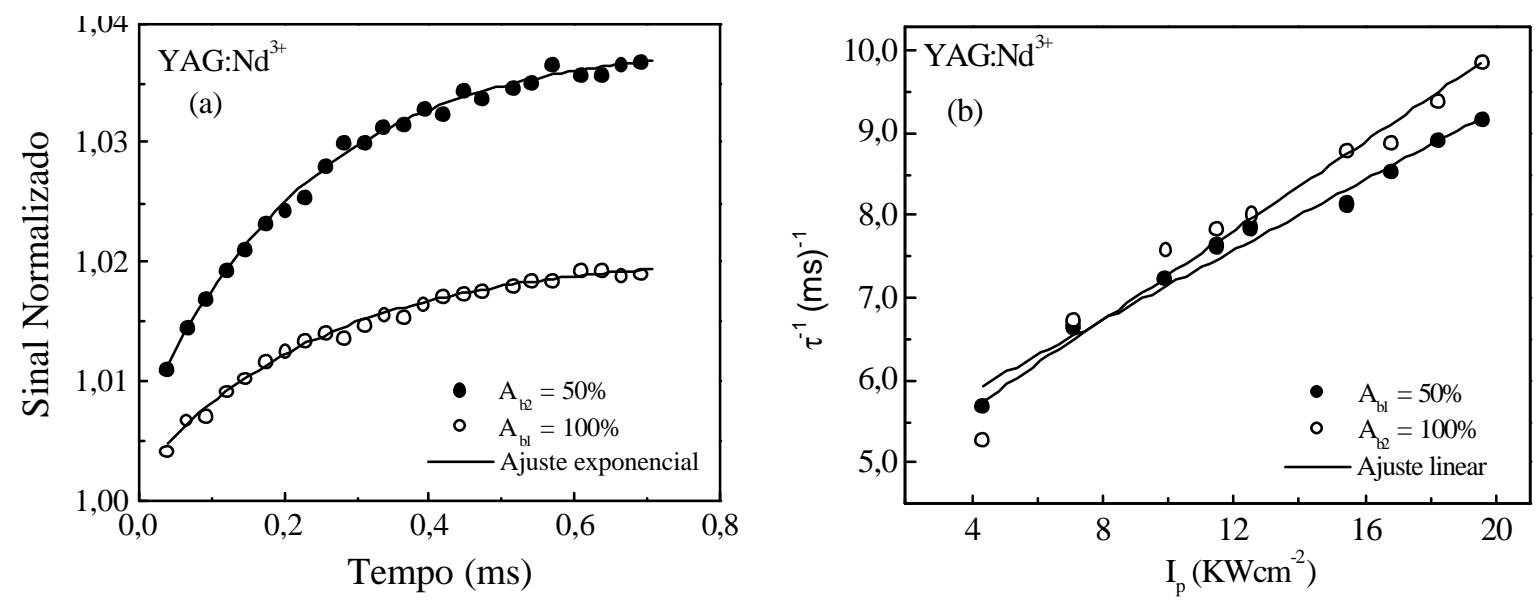

Figura 6.10 - (a) Dependência temporal do sinal de Zscan no YAG excitando em $\lambda=808 n m .(b)$ - valores de $\tau^{-1}$ versus $I_{p}$.

\begin{tabular}{ccccc}
\hline \hline YAG & $\begin{array}{c}\tau_{0} \mu s \\
\text { (medido) }\end{array}$ & $\begin{array}{c}I_{s} K W c m^{-2} \\
\text { (medido) }\end{array}$ & $\begin{array}{c}I_{s} K W c c^{-2} \\
\text { (calculado) }\end{array}$ & $\begin{array}{c}\tau_{0} \mu s \\
\text { (literatura) }\end{array}$ \\
\hline \hline $\mathrm{A}_{\mathrm{b} 1}=50 \%$ & $200 \pm 6$ & $20 \pm 2$ & 15,3 & 230 [51] \\
$\mathrm{A}_{\mathrm{b} 2}=100 \%$ & $220 \pm 12$ & $14,4 \pm 2$ & & \\
\hline
\end{tabular}

Tabela 6.2 - Tempo de vida da fluorescência, $\tau_{0}$, e intensidade de saturação, $I_{s}$ do $Y A G$.

Para as medidas no cristal de YLF, foi adotado o mesmo procedimento realizado no YAG. As medidas foram realizadas excitando com o laser de Ti:safira sintonizado em $802 \mathrm{~nm}$. Na Figura 6.11 temos as curvas da medida realizada com a abertura da fenda $\mathrm{A}_{\mathrm{b} 1}=50 \%$ (a) e $\mathrm{A}_{\mathrm{b} 2}=100 \%$ (b). A partir da análise dos resultados experimentais determinamos, $\mathrm{z}_{\mathrm{c}}=(0,50 \pm 0,01) \mathrm{cm}, \mathrm{n}_{2}=(10,5+\mathrm{i} 1,1) \times 10^{-10} \mathrm{~cm}^{2} / \mathrm{W}$, $? \mathrm{a}=(3,5 \pm 0,3) \times 10^{-26} \mathrm{~cm}^{3}$ e $? \mathrm{~s}=-(4,8 \pm 0,4) \times 10^{-21} \mathrm{~cm}^{2}$. No entanto o valor de $\Delta \alpha$ não está em concordância com o valor obtido por mistura de ondas (vide Figura 1.4 no Capítulo 1) ? a = $1,4 \times 10^{-26} \mathrm{~cm}^{3}$ [109]. 

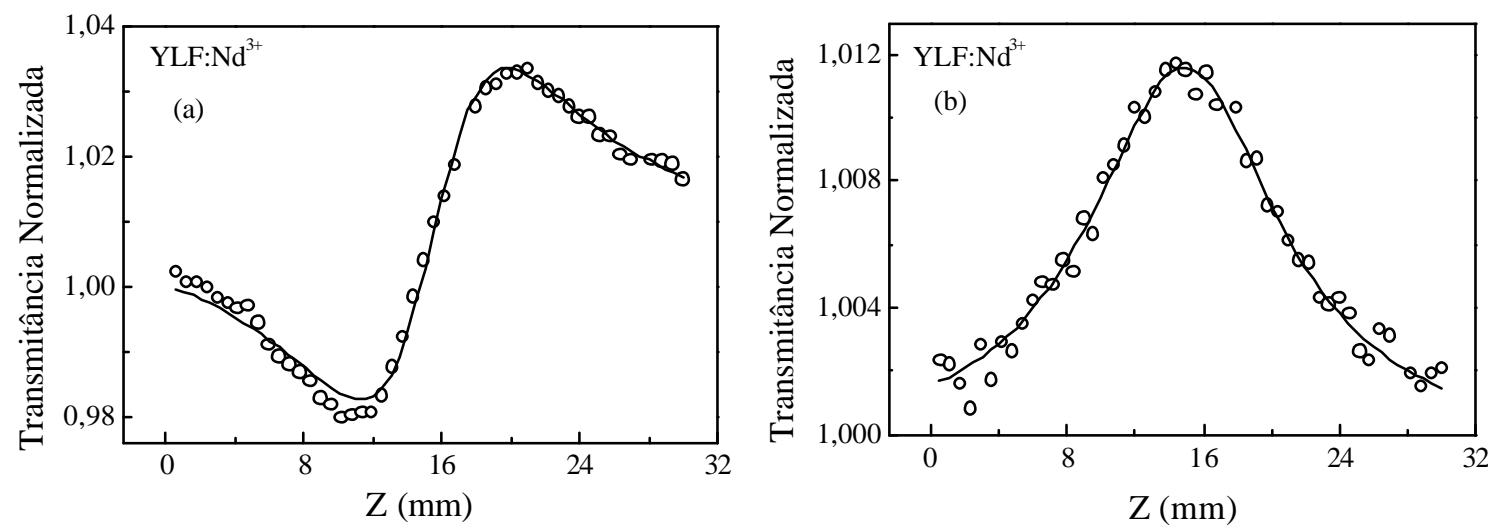

Figura 6.11 - Z-scan resolvido no tempo para o YLF . (a) fenda fechada $A_{b 1}=50 \%$, (b) fenda fechada $A_{b 2}=100 \%, f=820 \mathrm{~Hz}, P=250 \mathrm{~mW}$, linha contínua ajuste teórico feito com as expressões de Z-scan.

A Figura 6.12 mostra a variação do sinal da fenda fechada $\left(\right.$ ? $\left.\mathrm{T}_{\mathrm{p}-\mathrm{v}}\right)$ em função da intensidade, $I_{0}$. A partir do ajuste com a Eq. (6.7), foi obtido o parâmetro de saturação $I_{\mathrm{s}}=(43,4 \pm 8,0) \mathrm{KW} / \mathrm{cm}^{2}$. Este valor está em bom acordo com o calculado $\mathrm{I}_{\mathrm{s}}=41,8 \mathrm{KW} / \mathrm{cm}^{2}, \mathrm{com} \mathrm{s}=1,14 \times 10^{-20} \mathrm{~cm}^{2}[101]$ e $\mathrm{t}=520 \mu \mathrm{s}$ [111]. Na Figura 6.13 temos a variação da amplitude do sinal da fenda aberta em função do parâmetro de saturação $\mathrm{S}_{0}$. Ajustando os dados experimentais com a Eq. (6.18), obtivemos $?_{\text {up }}=(1,8 \pm 0,5) \times 10^{-16} \mathrm{~cm}^{3} / \mathrm{s}$. Este valor está em bom acordo com $?_{\text {up }}=(1,9 \pm 0,6) \times 10^{-16} \mathrm{~cm}^{3} / \mathrm{s}$ obtido com a técnica de LT (vide Tabela 5.3) e com $?_{\text {up }}=1,7 \times 10^{-16} \mathrm{~cm}^{3} / \mathrm{s}$ obtido por Guyot e colaboradores [101].

Na Tabela 6.3 apresentamos os resultados de $?_{\text {up }}$ comparando com os valores obtidos com a LT e os valores encontrados na literatura. 


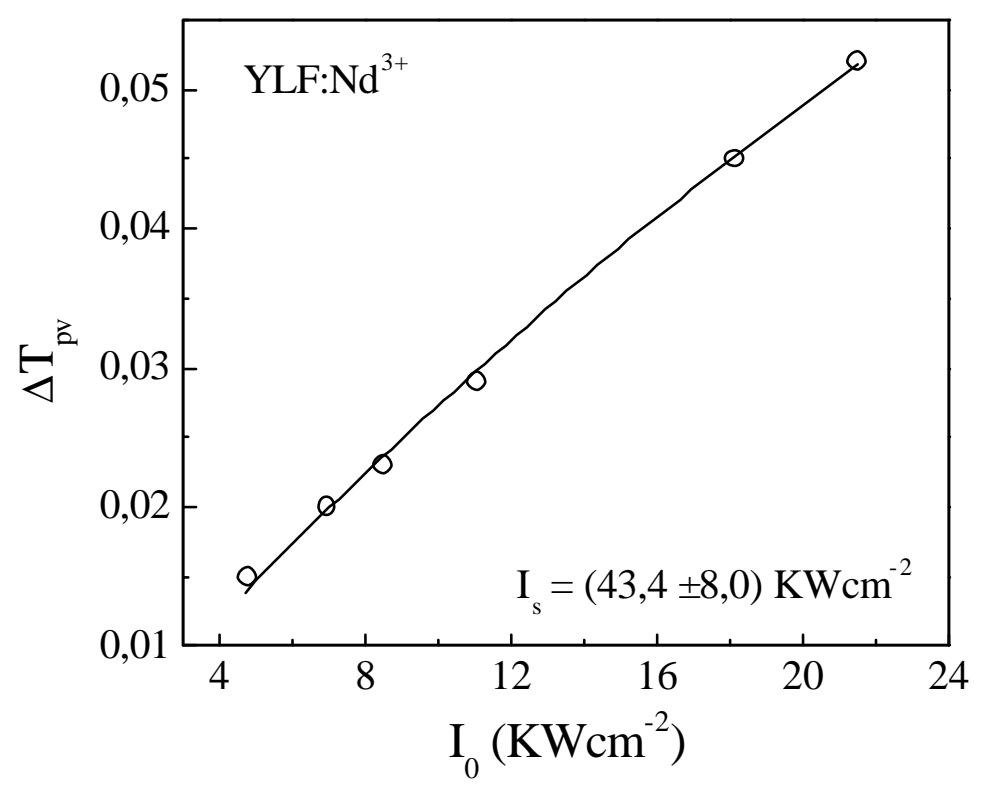

Figura 6.12 - Variação de ? $\mathrm{T}_{\mathrm{pv}}$ do cristal YLF em função da intensidade, $I_{0}$, com $A_{b 1}=50 \%$ e $\lambda=802 \mathrm{~nm}$. A linha sólida representa o ajuste feito com a Eq.(6.18).

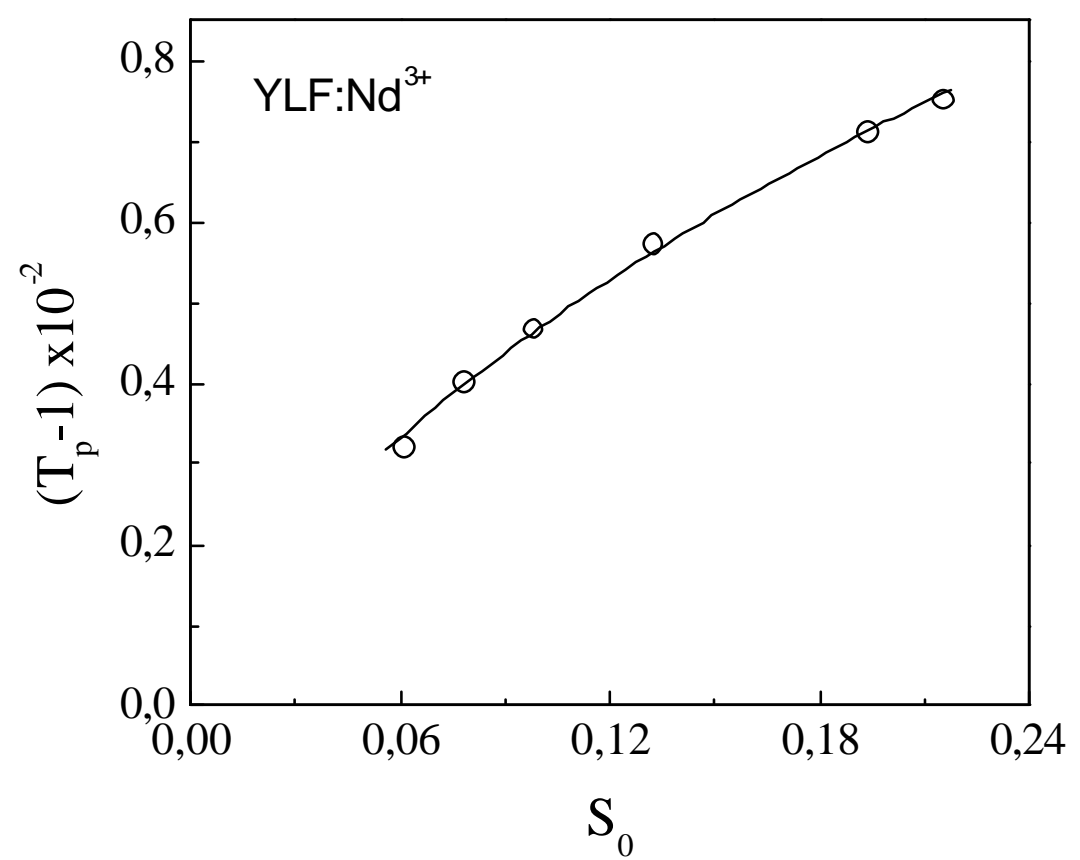

Figura 6.13 - Variação da amplitude do sinal da fenda aberta em função do parâmetro de saturação $S_{0}$, para o cristal YLF . Ajuste com a Eq.(6.18). $\beta=15,7 \pm 4,6$. 


\begin{tabular}{cccccc}
\hline \hline Amostra & $\%$ & $N_{0}$ & $\begin{array}{c}\gamma \\
\left(10^{20} \mathrm{~cm}^{-3}\right)\end{array}$ & $\begin{array}{c}\text { Método } \\
\left(10^{-16} \mathrm{~cm}^{3} / \mathrm{s}\right)\end{array}$ & \\
\hline \hline \multirow{2}{*}{$Y A G$} & 0,75 & 1,03 & 2,4 & Ref. & \\
& 1 & 1,38 & 2,8 & Fluorescência & Guyot e col. [101] \\
& $/ /$ & $/ /$ & 1,8 & & Chen e col. [112] \\
& $/ /$ & $/ /$ & 0,5 & Fluorescência & Guy e col. [49] \\
\hline \multirow{2}{*}{ YLF } & 1,2 & 1,7 & 1,9 & LT & \\
& $/ /$ & $/ /$ & 1,8 & Z-scan & \\
& 1,5 & 2,1 & 1,7 & Fluorescência & Guyot e col. [101] \\
\hline \multirow{2}{*}{ PGIZCa } & 3 & 6,5 & 1,9 & LT & \\
& $/ /$ & $/ /$ & 2,0 & Z-scan & \\
& 3 & 5,2 & 1,2 & LT & \\
& $/ /$ & $/ /$ & 1,1 & Z-scan & \\
& 1,5 & 2,5 & 0,46 & Fluorescência & Payne e col. [50] \\
\hline
\end{tabular}

Tabela 6.3 - Resultados do parâmetro de transferência de energia $\gamma$.

\subsection{Contribuições de Lente Térmica (LT) e Lente de População (LP) em vidros fluoretos.}

Os vidros fluoretos geralmente apresentam diferença de polarizabilidade ?a e condutividade térmica $K$ muito pequenos o que diminui o efeito de Lente de População (LP) e aumenta a Lente Térmica (LT), respectivamente. Consequentemente, o efeito de LP e LT podem dar contribuições de mesma magnitude ao sinal de Z-scan e para determinar ?a é preciso discriminar estes dois efeitos. Uma maneira de discriminar a contribuição dos efeitos de LP e LT, é realizar medidas de Zscan variando a frequência do chopper [8,9]. No entanto, no fluoretos observamos que a técnica de Zssan, variando a frequência do chopper, não pode por si só separar as contribuições dos efeitos de LP e LT. Nós introduzimos então um novo metodo para separar estas duas contribuições. Este metodo consiste em medir 
uma amostra referência onde supomos que exista apenas a contribuição de um dos efeitos.

Nos vidros fluoretos, as medidas de Zscan foram realizadas com um laser de $\mathrm{Ar}^{+}$sintonizado em $514 \mathrm{~nm}$, em ressonância com a linha de absorção ${ }^{4} \mathrm{G}_{7 / 2}+{ }^{2} \mathrm{G}_{9 / 2}$ do $\mathrm{Nd}^{3+}$ (vide Figura 3.7), para bombear o nível metaestável ${ }^{4} \mathrm{~F}_{3 / 2}$.

A Figura 6.14 mostra o resultado de uma medida de Z-scan com a fenda aberta $\left(\mathrm{A}_{\mathrm{b} 2}=100 \%\right)$ no vidro fluoroindato $(\operatorname{InSBZnGdN})$. Podemos notar que a transmitância aumenta no foco indicando que a transmissão aumenta (absorção decresce) quando o estado excitado é populado, ou seja, o material é um absorvedor saturável. Ajustando os dados experimentais com a Eq. (6.8), obtivemos $\mathrm{n}_{2}{ }^{\prime \prime}=-4,3 \times 10^{-12} \mathrm{~cm}^{2} \mathrm{~W}^{-1}$ e $? \mathrm{~s}=-1,4 \times 10^{-21} \mathrm{~cm}^{2}$.

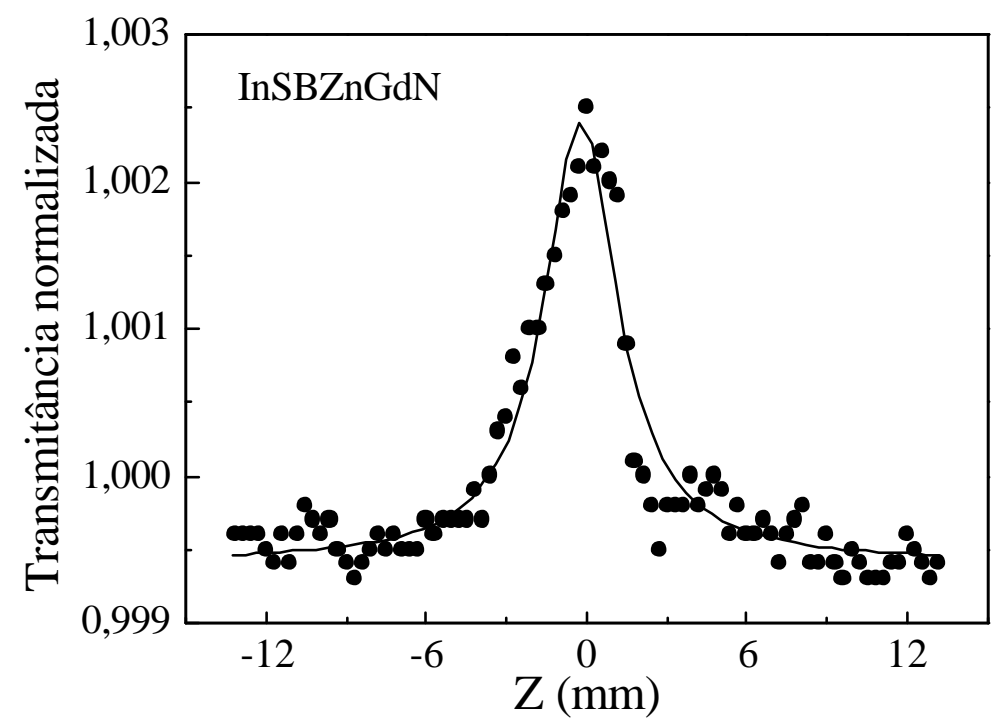

Figura 6.14 - Medida de Z-scan resolvido no tempo com abertura, $A_{b 2}=100 \%$, para o InSBZnGdN dopado com $\mathrm{Nd}^{3+}$, com $f=824 \mathrm{~Hz}, P=187 \mathrm{~mW}$ e $\lambda=514 \mathrm{~nm}$. A linha cheia indica o ajuste feito com a Eq. (6.8).

A Figura 6.15 mostra a medida de Z-scan resolvido no tempo, com $\mathrm{A}_{\mathrm{b} 1}=50 \%$, realizada com duas diferentes freqüências do "chopper" com a 
finalidade de separar os efeitos de LP e LT. Neste experimento o efeito de LP é mais rápido do que o de $\mathrm{LT}$, tal que na alta freqüência do "chopper" a curva indica uma não linearidade positiva, indicando que o efeito de LP predomina. Seguindo o procedimento usual da técnica de Z-scan, a curva de LP mostrada, foi normalizada pela medida realizada com abertura $\mathrm{A}_{\mathrm{b} 2}=100 \%$, devido a absorção do estado excitado [12, 14]. Em nossa medida, a intensidade axial de pico foi $\mathrm{I}_{0}=4,0 \times 10^{4} \mathrm{~W} / \mathrm{cm}^{2}$, a qual é muito menor do que a intensidade de saturação $I_{s}=2,5 \times 10^{5} \mathrm{~W} / \mathrm{cm}^{2}$, tal que o efeito de saturação pode ser desprezado.

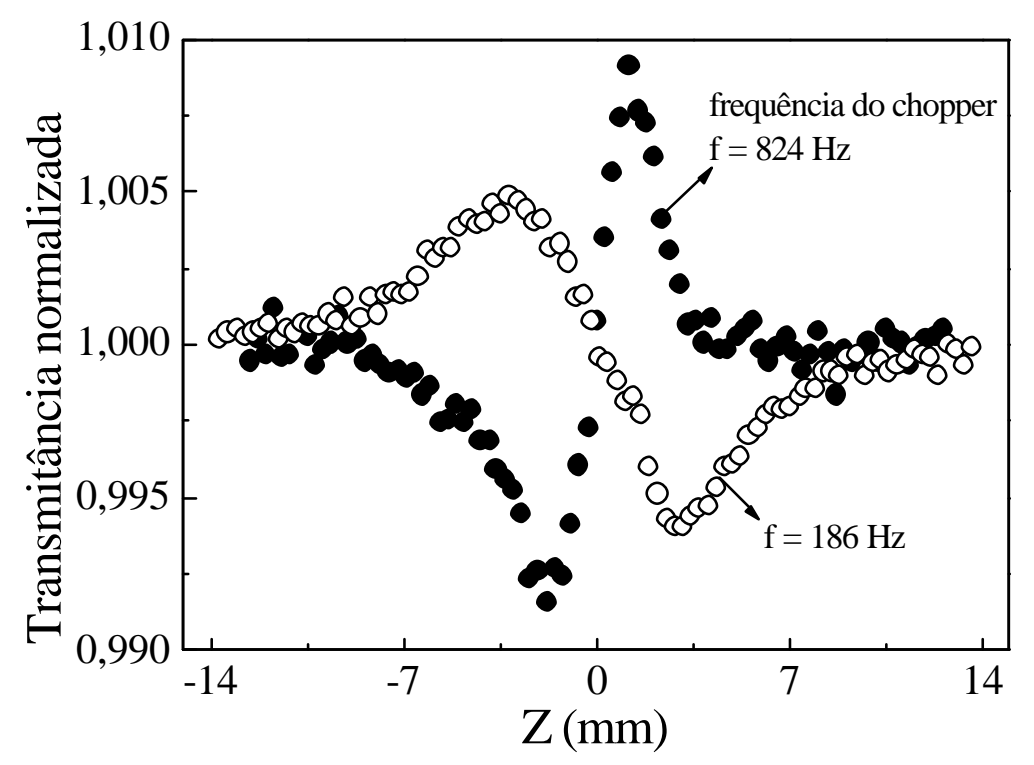

Figura 6.15 - Z-scan resolvido no tempo com $\lambda=514 \mathrm{~nm}$ e abertura $A_{b 1}=50 \%$, para o InSBZnGdN dopado com $\mathrm{Nd}^{3+}$. (•?) freqüência do "chopper" $f=180 \mathrm{~Hz}$, $P=230 \mathrm{~mW}$, (o) $f=824 \mathrm{~Hz}$ e $P=187 \mathrm{~mW}$.

Para baixa frequiência do "chopper" (indicado por $\mathrm{f}=186 \mathrm{~Hz}$ na Figura 6.15), a curva é invertida, indicando que $? \mathrm{~s}<0$ (sendo ? s a variação do caminho ótico). Este resultado indica que neste regime observamos o efeito de LT, o qual é sabido ser negativo em vidros fluoretos, que apresentam ds $/ \mathrm{dT}<0[5,7]$. No caso do efeito 
de LT, a distância entre o pico e o vale é $? \mathrm{Z}_{\mathrm{pv}} \sim 3,4 \mathrm{z}_{0}$, o qual é duas vezes maior do que o devido ao efeito de LP $[14,57]$. Este aumento em ? $\mathrm{Z}_{\mathrm{pv}}$ é uma conseqüência do aumento no perfil do índice de refração, ?n(r), na LT devido a difusão de calor.

Nos vidros fluoretos ZBLAN, YABC, InSBZnGaN e PGIZCa, não observamos inversão na curva quando variamos a freqüência do "chopper". A Figura 6.16 mostra o resultado no ZBLAN o qual indica que a contribuição do efeito térmico é maior (em módulo) do que a contribuição eletrônica em ambas frequiências. Provavelmente as contribuições térmica e eletrônica têm magnitude similar e sinais opostos, tal que eles quase se cancelam.

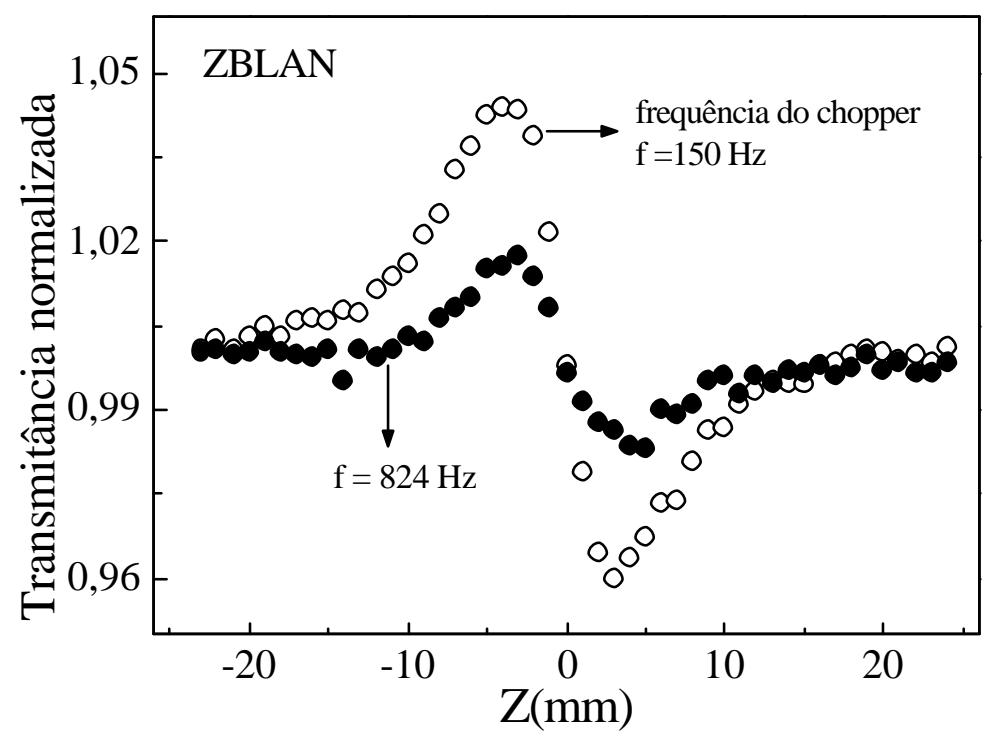

Figura 6.16 - Z-scan resolvido no tempo $\operatorname{com} \lambda=514 \mathrm{~nm}$ e abertura $A_{b 1}=50 \%$, para o vidro ZBLAN dopado com $\mathrm{Nd}^{3+}$. (O) freqüência do “chopper" $f=150 \mathrm{~Hz}$, $P=145 \mathrm{~mW},(\bullet \rho)=824 \mathrm{~Hz}$, e $P=245 \mathrm{~mW}$.

Para uma melhor compreensão deste problema, realizamos medidas com amostras dopadas com $\mathrm{Co}^{2+}$, uma vez que neste material é conhecido [7] que praticamente toda a energia absorvida pela amostra é convertida em calor, tal que $\varphi=1$. Nos vidros dopados com $\mathrm{Co}^{2+}$, não existe população no estado metaestável, e 
portanto não há contribuição eletrônica $\mathrm{n}_{2}$ (no regime $\mathrm{cw}$ ). Amostras dopadas com baixa concentrações de $\mathrm{Co}^{2+}$ e $\mathrm{Nd}^{3+}$ apresentam as mesmas propriedades termoóticas (particularmente ds/dT e difusividade térmica, $D$ ), de tal forma que podemos

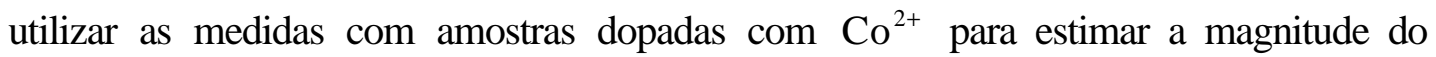
efeito térmico nas amostras dopadas com $\mathrm{Nd}^{3+}$.

A Figura 6.17 mostra a curva de Z-scan para o vidro fluoroindato (InSBZnGdN ) dopado com $0,2 \%$ de $\mathrm{Co}^{2+}$, realizada nas mesmas condições experimentais como na Figura $6.15(\mathrm{f}=824 \mathrm{~Hz})$, onde para $\mathrm{P}=100 \mathrm{~mW}$ e $\mathrm{A}=0,8 \mathrm{~cm}^{-1}$ nós encontramos $? \mathrm{~T}_{\mathrm{pv}}=-7,0 \times 10^{-3}$ (o sinal negativo indica não linearidade negativa).

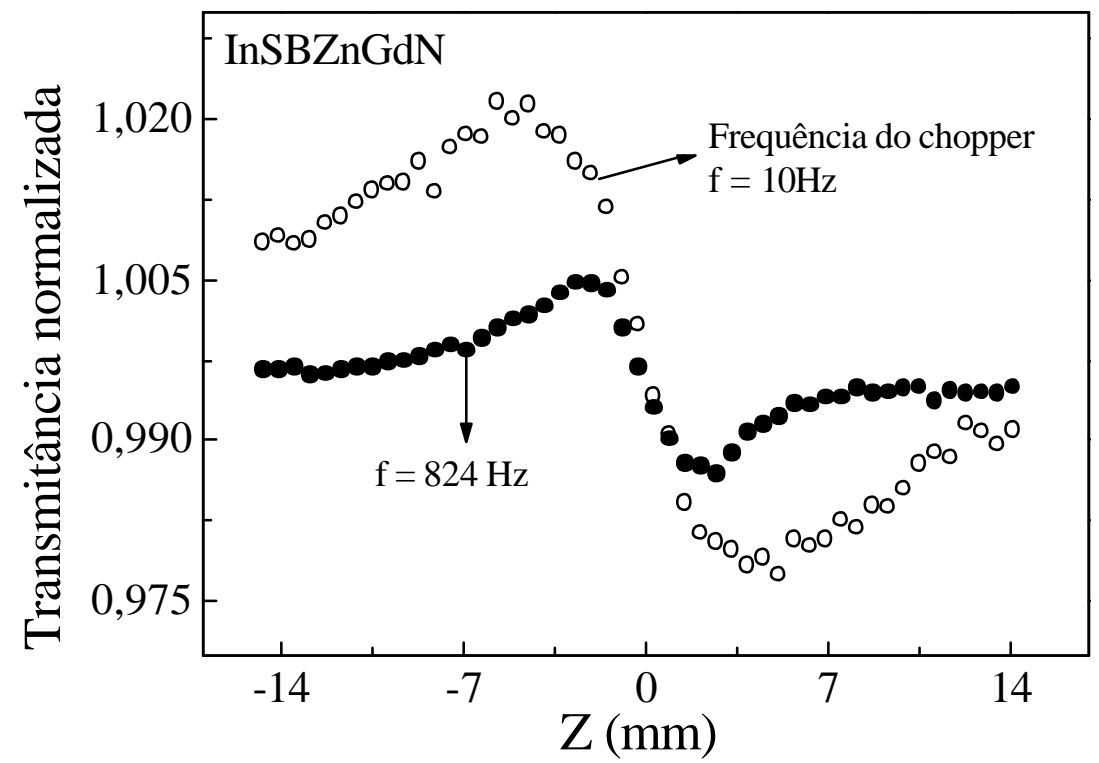

Figura 6.17 - Z-scan resolvido no tempo com $\lambda=514 \mathrm{~nm}$ e abertura $A_{b 1}=50 \%$, para o InSBZnGdN dopado com CoF . $_{2}(\bullet$ P Freqüencia do "chopper" $f=824 \mathrm{~Hz}$, $P=100 \mathrm{~mW}$, (o) $f=10 \mathrm{~Hz}$ e $P=100 \mathrm{~mW}$.

Relembrando que o sinal devido a não-linearidade térmica é proporcional a $\operatorname{PA} \varphi$, a contribuição térmica para a Figura $6.15(f=824 \mathrm{~Hz})$ foi estimada sendo igual a $\left.? \mathrm{~T}_{\mathrm{pv}}\right)_{\mathrm{th}}=-1,3 \times 10^{-2}$. A curva de Z-scan da Figura $6.15(f=824 \mathrm{~Hz})$ pode 
ser imaginada como uma superposição de duas curvas, ou seja, a curva medida ? $\left.\mathrm{T}_{\mathrm{pv}}\right)_{\mathrm{exp}}$, é dada por

$$
\left.\left.\left.? \mathrm{~T}_{\mathrm{pv}}\right)_{\exp }=? \mathrm{~T}_{\mathrm{pv}}\right)_{\mathrm{pop}}+? \mathrm{~T}_{\mathrm{pv}}\right)_{\mathrm{th}}
$$

sendo ? $\mathrm{T}_{\mathrm{pv}}$ a diferença de transmissão entre o pico e o vale e o subíndice se refere as contribuições de LP ( $p o p$ ) e de LT $(t h)$. Os subíndices exp, pop e th, referem-se ao valor experimental, a contribuição de LP e a contribuição de LT, respectivamente.

Desde que na Figura $6.15(f=824 \mathrm{~Hz})$ ? $\left.\mathrm{T}_{\mathrm{pv}}\right)_{\exp }=+1,7 \times 10^{-2}$, pela Eq. $\begin{array}{llllll}\text { é } & \text { possível } & \text { obter efeito } & \text { como }\end{array}$ $\left.? \mathrm{~T}_{\mathrm{pv}}\right)_{\mathrm{pop}}=(1,7+1,3) \times 10^{-2}=3,0 \times 10^{-2}$, onde $\left.? \mathrm{~T}_{\mathrm{pv}}\right)_{\mathrm{th}}=-1,3 \times 10^{-2}$. Utilizando as Eqs. (6.7) e (1.20) os valores de $\mathrm{n}_{2}$ e ? a podem ser obtidos.

O mesmo procedimento foi utilizado para estimar ? $\left.\mathrm{T}_{\mathrm{pv}}\right)_{\text {pop }}$ para as outras amostras, cujos resultados estão mostrados na Tabela 6.4. Por exemplo, na Figura $6.16\left(\text { ZBLAN ) nós obtivemos } ? \mathrm{~T}_{\mathrm{pv}}\right)_{\exp }=-3,5 \times 10^{-2}$. A partir da medida com uma amostra de ZBLAN dopada com $\mathrm{Co}^{2+}$ (similar a Figura 6.17 com $824 \mathrm{~Hz}$ ), nós estimamos $\left.? \mathrm{~T}_{\mathrm{pv}}\right)_{\mathrm{th}}=-7,0 \times 10^{-2}$, tal que pela Eq. (6.19), nós obtivemos $\left.? \mathrm{~T}_{\mathrm{pv}}\right)_{\mathrm{pop}}=3,5 \times 10^{-2}$. O mesmo procedimento foi utilizado para o PGIZCa, YABC e InSBZnGaN .

A principal dificuldade em medir $? \mathrm{a}<10^{-25} \mathrm{~cm}^{3}$ é discriminar as contribuições dos efeitos de LT de LP. Para analisar os resultados, nós definimos o fator $\mathrm{Q}=$ ? $\left.\mathrm{T}_{\mathrm{pv}}\right)_{\mathrm{pop}} /$ ? $\left.\mathrm{T}_{\mathrm{pv}}\right)_{\mathrm{th}}$. Podemos notar que $\mathrm{Q}$ depende da freqüência do "chopper" que define o tempo final, $t_{f}$, onde a medida é feita. Esta definição é mostrada na Figura 6.15 onde para baixa frequiência $\left.(186 \mathrm{~Hz}) \quad ? \mathrm{~T}_{\mathrm{pv}}\right)_{\mathrm{pop}}<$ ? $\left.\mathrm{T}_{\mathrm{pv}}\right)_{\mathrm{th}}$ ou 
$\mathrm{Q}<1$ e para alta freqüência $(824 \mathrm{~Hz}) \mathrm{Q}>1$. Este aumento de $\mathrm{Q}$ com a freqüência depende de $t_{c}$ e $\tau$, que determinam os tempos de resposta térmica e eletrônica, respectivamente. No regime estacionário (para $t>t_{c}$ e $\tau$ ) ? $\left.\mathrm{T}_{\mathrm{pv}}\right)_{\mathrm{pop}} \sim 0,406$ ?, 0 $[12,14]$ e $\left.? \mathrm{~T}_{\mathrm{pv}}\right)_{\mathrm{th}} \sim 1,06$ ? [57]. Portanto chamando $\mathrm{Q}(\infty)$ o valor de $\mathrm{Q}$ no estado estacionário (para $t \gg t_{c}$ e $\tau$ ), das Eqs. (2.5) e (6.7) $\mathrm{Q}(\infty) \propto \mathrm{t}$ ? a k/( $\mathrm{w}_{0}^{2} \mathrm{ds} / \mathrm{dT}$ ). Podemos notar que este fator tem uma forte dependência com matriz e com a configuração experimental devido ao parâmetro $\mathrm{w}_{0}$. Por exemplo, $\mathrm{Q}(\infty)=0,97$ e 0,11 pode ser estimado para o InSBZnGdN e ZBLAN, respectivamente.

O InSBZnGdN foi a única amostra onde observamos a inversão da curva de Z-scan (Figura 6.15). Para esta amostra nós obtivemos ? $\left.\mathrm{T}_{\mathrm{pv}}\right)_{\mathrm{pop}} \sim 1,8$ ? $\left.\mathrm{T}_{\mathrm{pv}}\right)_{\exp }$ $(\mathrm{Q}=2,2)$ para $824 \mathrm{~Hz}$, de maneira que $\mathrm{Q}$ é o dobro comparado a $\mathrm{Q}(\infty)$ Neste caso, embora tenhamos Q $>1$, a contribuição térmica não pode ser desprezada. Ou seja, a técnica de discriminação temporal não é suficiente para eliminar a contribuição térmica.. A Tabela 6.4 mostra a razão $\left.\mathrm{Q}=? \mathrm{~T}_{\mathrm{pv}}\right)_{\mathrm{pop}} /$ ? $\left.\mathrm{T}_{\mathrm{pv}}\right)_{\text {th }}$ obtido para todos os vidros estudados com frequiência $f=824 \mathrm{~Hz}$. Pode ser observado que $\theta$ é proporcional ao produto $\mathrm{k}^{-1} \mathrm{ds} / \mathrm{dT}$ (também listados na Tabela 6.4). O ZBLAN apresenta o maior $\mathrm{k}^{-1} \mathrm{ds} / \mathrm{dT}$ e o menor fator $\mathrm{Q}$.

Nestes experimentos nós notamos que a forma da curva de Z-scan depende da escala de tempo experimental, a qual é relacionada à freqüência do "chopper", mesmo quando somente o efeito térmico está presente como na Figura 6.17 para ZBLAN $: \mathrm{Co}^{2+}$. Nós observamos que para freqüência alta $(824 H z)$ ? $\mathrm{Z}_{\mathrm{pv}}$ é aproximadamente a metade do valor da curva de baixa freqüência $(10 \mathrm{~Hz})$. Esta 
diferença na forma da curva do Zscan é devido ao efeito da difusão de calor. Para frequiência alta (medidas realizadas com $t<t_{c}$ ) o perfil de temperatura ?T(r, t) deve ser próximo do perfil de intensidade gaussiano dado por $I(r)=I(0) \exp \left[-2 \mathrm{r}^{2} / \mathrm{pw}_{0}^{2}\right]$ que conduz a $? \mathrm{Z}_{\mathrm{pv}}=1,7 \mathrm{z}_{0}$ de acordo com Sheik-Bahae e colaboradores [14]. No caso estacionário o perfil de temperatura ?T(r,t) é muito mais largo que o perfil de intensidade. A curva de baixa frequiência se assemelha ao caso estacionário onde $? \mathrm{Z}_{\mathrm{pv}}=3,4 \mathrm{z}_{0}$ como mostrado por Sheldon e colaboradores [57].

Nosso resultado de $? \mathrm{a}=(2,7 \pm 0,8) \times 10^{-26} \mathrm{~cm}^{3}$ para o vidro fluoroindato (InSBZnGdN ) e $1 \times 10^{-26} \leq ? \mathrm{a} \leq 3 \times 10^{-26} \mathrm{~cm}^{3}$ para os vidros fluoretos, é similar aos valores de ? a para cristais fluoretos e vidros fluorofosfatos, obtidos por Powell e colaboradores usando a técnica de mistura de quatro ondas [3]. 



\begin{tabular}{|c|c|c|c|c|c|c|c|c|c|}
\hline Amostras & $\begin{array}{c}\tau \\
(\mu \mathrm{s}) \\
\pm 5 \% \\
\end{array}$ & $\begin{array}{c}\mathrm{A} \\
\left(\mathrm{cm}^{-1}\right) \\
\pm 4 \% \\
\end{array}$ & $\begin{array}{c}\mathrm{k}^{-1} \mathrm{ds} / \mathrm{dT} \\
\left(10^{-4} \mathrm{cmK}^{-1}\right) \\
\pm 15 \%[7] \\
\end{array}$ & $\begin{array}{c}\mathrm{Q} \\
(f=824 H z) \\
\pm 30 \% \\
\end{array}$ & $\begin{array}{c}\mathrm{s} \\
\left(10^{-21} \mathrm{~cm}^{2}\right) \\
\pm 5 \% \\
\end{array}$ & $\begin{array}{c}\mathrm{n}_{2}{ }^{\prime} \\
\left(10^{-10} \mathrm{~cm}^{2} / \mathrm{W}\right) \\
\pm 30 \% \\
\end{array}$ & $\begin{array}{c}\mathrm{n}_{2} " \\
\left(10^{-12} \mathrm{~cm}^{2} / \mathrm{W}\right) \\
\pm 10 \% \\
\end{array}$ & $\begin{array}{c}? \mathrm{~s} \\
\left(10^{-21} \mathrm{~cm}^{2}\right) \\
\pm 15 \% \\
\end{array}$ & $\begin{array}{c}? \mathrm{a} \\
\left(10^{-26} \mathrm{~cm}^{3}\right) \\
\pm 30 \% \\
\end{array}$ \\
\hline 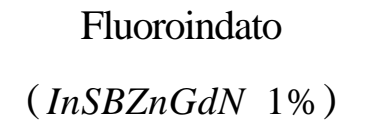 & 470 & 0,64 & 2,1 & 2,2 & 3,3 & 1,8 & $-4,3$ & $-1,4$ & 2,7 \\
\hline $\begin{array}{l}\text { Fluoroaluminato } \\
\left(\begin{array}{ll}Y A B C & 2 \%\end{array}\right)\end{array}$ & 430 & 1,24 & 2,4 & 0,64 & 3,2 & 1,4 & 10,0 & 1,8 & 1,3 \\
\hline $\begin{array}{c}\text { Fluoroindato } \\
\left(\begin{array}{ll}P G I Z C a & 2 \%\end{array}\right)\end{array}$ & 285 & 1,36 & 2,3 & 0,74 & 3,4 & 2,0 & 3,6 & 0,9 & 2,3 \\
\hline
\end{tabular}

Tabela 6.4 - Principais parâmetros e resultados de Z-scan em vidros fluoretos dopados com $\mathrm{Nd}^{3+} \lambda=514 \mathrm{~nm}$. 


\section{Capítulo 7}

\section{Conclusão}

Nesta tese apresentamos uma série de resultados envolvendo o efeito térmico e/ou o efeito não-linear em sólidos dopados, onde nosso objetivo principal era estudar:

1) A difusividade térmica.

2) Eficiência quântica em sólidos dopados com diferentes concentrações de $\mathrm{Nd}^{3+}$

3) Variação do caminho ótico com a temperatura (ds/dT).

4) Processos de transferência de energia por "upconversion Auger" e relação cruzada.

5) Separação entre as propriedades não lineares eletrônica e térmica.

Com relação ao primeiro item, nós determinamos através da técnica de LT a difusividade térmica dos cristais: YAG, $\mathrm{YVO}_{4}, \mathrm{BTO}, \mathrm{BSO}$; do vidro calcohaleto e do filme fino PPV. Para o YAG nós determinamos $\mathrm{D}=(4,80 \pm 0,05) \times 10^{-2} \mathrm{~cm}^{2} \mathrm{~s}^{-1}$, estando em bom acordo com o valor encontrado na literatura $\mathrm{D}=4,6 \times 10^{-2} \mathrm{~cm}^{2} \mathrm{~s}^{-1}$ [51]. No vidro calcohaleto, a difusividade térmica obtida $\mathrm{D}=1,3 \times 10^{-3} \mathrm{~cm}^{2} \mathrm{~s}^{-1}$ é aproximadamente a metade da difusividade dos vidros fluoretos $\left(\sim 2,5 \times 10^{-3} \mathrm{~cm}^{2} \mathrm{~s}^{-1}\right)$ [7] e um quarto do vidro aluminato (LSCAS ) $\sim 5,5 \times 10^{-3} \mathrm{~cm}^{2} \mathrm{~s}^{-1}$ [113]. Nos cristais BTO e BSO, apesar pertencerem a mesma família dos silenitas, observamos uma diferença de $\sim 17 \%$ entre os valores obtidos para a difusividade térmica. No cristal $\mathrm{YVO}_{4}$ observamos uma pequena diferença no valor da difusividade com a 
orientação. No entanto, esta diferença já era esperada, uma vez que, neste tipo de material o valor da difusividade depende da orientação cristalográfica [63-65].

Com relação ao segundo item, medida de eficiência quântica em sólidos dopados com diferentes concentrações de $\mathrm{Nd}^{3+}$, apresentamos um estudo de eficiência quântica em sólidos dopados com $\mathrm{Nd}^{3+}$. Analisamos o comportamento da eficiência quântica com o aumento da concentração de íons de $\mathrm{Nd}^{3+}$ e comparamos os resultados obtidos através da técnica de LT com os obtidos por outras técnicas, tais como: fotoacústica, esfera integradora, luminescência e calorimetria.

Para medir a eficiência quântica, nós utilizamos duas variações do método de LT. Primeiramente usamos uma amostra referência, onde supomos que toda luz absorvida era convertida em calor, tal que a eficiência quântica era zero, isto nos permitiu determinar o parâmetro $\left(1 / \mathrm{k} ?_{\mathrm{p}}\right) \mathrm{ds} / \mathrm{dT}$. No segundo método determinamos a eficiência quântica, $\eta$, medindo o parâmetro $\mathrm{T}$ variando o comprimento de onda de excitação. O bom acordo entre os resultados obtidos através dois métodos, indica que o método variando o comprimento de onda, é um método confiável e tem a vantagem de fornecer o valor absoluto da eficiência quântica da fluorescência sem a necessidade de uma amostra referência. Portanto, este método parece ser superior em relação ao primeiro, no estudo de materiais altamente transparente e com baixo efeito de LT.

Mostramos ainda que a técnica de LT também pode ser utilizada para medir a eficiência quântica de filmes poliméricos. Nós medimos o filme PPV (p-fenilenovinileno), termicamente convertido a baixa temperatura, cujo resultado foi $?=0,85 \pm 0,15$. Da literatura é esperado que a eficiência quântica seja maior do que $50 \%$ em filmes parcialmente conjugados $[90,91]$. Este nosso resultado indica que o 
PPV estudado apresenta poucos defeitos estruturais (relacionados com canais não radiativos, como grupos carbonila).

Quanto ao terceiro item, estuda da variação do caminho ótico com a temperatura (ds/dT), determinamos ds/dT em vários materiais (vidros e cristais) e comparamos os resultados obtidos com o valor calculado através da Eq. (4.5), que considera a contribuição de $\mathrm{dn} / \mathrm{dT}$, do coeficiente de expansão térmica e o efeito fotoelástico. Para alguns materiais aqui estudados, os parâmetros fotoelásticos não foram encontrados na literatura, de forma que para estimar a contribuição do termo devido ao efeito fotoelástico, fizemos algumas aproximações. Para os cristais fluoretos usamos os valores do $\mathrm{CaF}_{2}$ e para o cristal $\mathrm{YVO}_{4}$ usamos o valor do YAG . Apesar desta aproximação ser um tanto grosseira, ela serviu para dar uma idéia da contribuição do efeito fotoelástico. Notamos que, a maior contribuição para o parâmetro ds/dT se deve ao termo da expansão térmica ? ${ }_{\mathrm{a}}$, sendo este muito maior do que o termo devido ao efeito fotoelástico $?_{\mathrm{q}}$. Nos cristais $\mathrm{YVO}_{4}, \mathrm{YLF}$, LiSAF e LiSGaF, por serem uniaxiais, tal que os parâmetros $\alpha, k$ e $D$ assumem diferentes valores de acordo com a orientação [63-65], as medidas de LT foram realizadas com o feixe de prova polarizado com o objetivo de verificar-mos o comportamento do parâmetro ds/dT com a orientação do cristal.

Devido a forte absorção em 632nm no vidro calcohalet o (comprimento de onda utilizado como feixe de prova) as medidas de LT foram realizadas com a configuração de feixe único. Neste material como não temos conhecimento do valor do calor específico e nem da condutividade térmica, $\mathrm{k}$. consequentemente não conseguimos determinar o parâmetro ds/dT, mas apenas ds/dQ, que representa a variação do caminho ótico com o calor depositado por unidade de volume. 
No quarto item, estudo dos processos de transferência de energia por "upconversion Auger" e relação cruzada, mostramos que a técnica de LT também pode ser utilizada como um método alternativo para estudar os processos de transferência de energia "upconversion Auger" $\left(?_{\text {up }}\right)$ e relaxação cruzada $\left(?_{\text {rc }}\right)$ em sólidos dopados. Apesar do grande número de trabalhos que tratam dos processos de transferência de energia em sólidos dopados, ainda não se encontra na literatura resultados experimentais dos parâmetros que descrevem estes processos, em função da concentração para um mesmo conjunto de amostras. Neste trabalho, nós estudamos cristais dopados com $\mathrm{Cr}^{3+}$ e $\mathrm{Nd}^{3+}$ e os vidros fluorozirconato (ZBLAN) e fluoroindato (PGIZCa ) com diferentes concentrações de $\mathrm{Nd}^{3+}$. Por exemplo, em uma amostra de ZBLAN (com concentração $2,5 \times 10^{20}$ íns $/ \mathrm{cm}^{3}$ de $\mathrm{Nd}^{3+}$ ), Payne e colaboradores [50] obtiveram por meio de medidas transientes um $?_{\text {up }}=(4,6 \pm 1,4) \times 10^{-17} \mathrm{~cm}^{3} / \mathrm{s}$. Para isto eles usaram um $\mathrm{d}=0,63$, onde $\mathrm{d}=\mathfrak{B S}$ $\left(\mathrm{d}=\mathrm{N}_{\mathrm{ex}}\right.$ ? up $\mathrm{t}$ ). No entanto, nós medimos uma amostra de ZBLAN similar ao medido por Payne com um $\mathrm{N}_{\mathrm{ex}}$ muito menor, correspondendo a um $\mathrm{d} \sim 0,015-0,03$, que é um valor muito menor que o utilizado pelos autores. Isto mostra que o nosso método é muito mais sensível ( 20 vezes). Nós determinamos um $?_{\text {up }}=6,0 \times 10^{-17} \mathrm{~cm}^{3} / \mathrm{s}$ para uma amostra de ZBLAN com $\mathrm{N}_{0}=2,5 \times 10^{20}$ íns $/ \mathrm{cm}^{3}$, a mesma concentração usada por Payne e colaboradores. A discrepância entre os resultados pode ser atribuída aos diferentes métodos utilizados na determinação do parâmetro "upconversion" e considerando todos os erros envolvidos, estes números podem ser considerados em bom acordo.

Para realizar o quinto item, estudo da separação entre as propriedades não lineares eletrônica e térmica, nós utilizamos a técnica de Z-scan resolvida no tempo. 
Esta técnica nos possibilitou discriminar temporalmente, através da freqüência do "chopper", a contribuição do efeito de LP e do efeito de LT, uma vez que o tempo de resposta característico da LP é $\mathrm{t}$ e o tempo do efeito térmico é $\mathrm{t}_{\mathrm{c}}=\mathrm{w}_{0}^{2} / 4 \mathrm{D}$. Determinamos também a diferença de polarizabilidade ?a, a diferença de seção de choque de absorção ?s e a intensidade de saturação $I_{s}$ dos cristais YAG e YLF . Mostramos ainda que, a técnica de Zscan também pode ser utilizada para determinar os parâmetros de transferência de energia, através do monitoramento do sinal da amplitude da fenda aberta em função da potência de bombeio. Para isto, nós encontramos uma expressão, para a fenda aberta, que considera o efeito da transferência de energia.

Nos vidros fluoretos, observamos que a técnica de Z-scan resolvida no tempo não pode por si só separar as contribuições dos efeitos de LP e LT. No entanto, nós introduzimos uma nova metodologia para separar estas duas contribuições. Nós realizamos medidas em amostras dopadas com $\mathrm{Co}^{2+}$, onde é conhecido que toda energia absorvida é convertida em calor, tal que $\varphi=1$, e além disso não existe população no estado metaestável, fazendo com que não haja contribuição do efeito de LP. Comparando as medidas as amostras de $\mathrm{Co}^{2+}$ com as de $\mathrm{Nd}^{3+}$, conseguimos obter uma estimativa da contribuição do efeito de LT. Os valores de ?a's obtidos variaram entre 1 e $3 \times 10^{-26} \mathrm{~cm}^{3}$. No entanto, a necessidade de uma amostra de referência faz com que o método seja uma medida indireta e haja um aumento na sua incerteza experimental. 


\subsection{Perspectivas de Trabalhos futuros.}

Como possibilidades de trabalhos futuros, podemos destacar os seguintes tópicos:

$>$ Aplicar a técnica de Lente Térmica para determinar a eficiência quântica da fluorescência em filmes fino.

Nas últimas décadas uma nova classe de materiais denominados semicondutores orgânico emissores de luz tem sido pesquisada por apresentar propriedades óticas e eletrônicas similares às dos semicondutores inorgânicos. A grande vantagem destes novos materiais é que podem ser produzidos na forma de filmes finos e ultrafinos a um custo extremamente baixo. Isto os torna objeto de interesse tanto do ponto de vista tecnológico (dispositivos otoeletrônicos e eletrônicos), quanto do ponto de vista da física fundamental e da ciência dos materiais (estrutura eletrônica, excitações elementares, transporte e transferência de energia, bem como as propriedades e formação de filmes finos). Dentre os materiais orgânicos destacam-se os polímeros conjugados e macromoléculas, por apresentarem propriedades particulares de emissão de luz.

Uma característica fundamental destes materiais orgânicos do ponto de vista físico e químico é sua a habilidade de transferir energia absorvida de um sítio específico para outro induzindo processos de transporte de portadores, luminescência, reações fotoquímicas e outros. Do mesmo modo, os polímeros semicondutores também apresentam esta riqueza de fenômenos. O estudo detalhado dos processos radiativos e não radiativos nesses polímeros, bem como a habilidade de se fazer predições e estimativas, são de fundamental importância para elucidar a natureza e os mecanismos das transições óticas nesses materiais. 
Investigar o efeito da transferência de energia nos cristais YAG YLF, variando a concentração de íons de $\mathrm{Nd}^{3+}$. No YAG tentar compreender o fenômeno que ocorre com o aumento da potência de excitação, como apresentado no Capítulo 5.

Realizar medidas de Z-scan no fluoretos e outros materiais dopados com $\mathrm{Nd}^{3+}$ excitando em 800nm, para determinar o índice de refração nãolinear $\left(\mathrm{n}_{2}\right)$ e a diferença de polarizabilidade (? a )

Estas medidas em ? 800nm são importantes na determinação de $\mathrm{n}_{2}$ e ?a para estes materiais, uma vez que, no infravermelho em ressonância com o nível ${ }^{4} \mathrm{~F}_{3 / 2}$, é possível diminuir a contribuição do efeito térmico, já que a fração de energia que é absorvida e convertida em calor pela amostra, $\varphi$, diminui com o comprimento de onda de excitação. 


\section{Referências}

[1] M. M. Bubnov, A. B.Grudinin, E. M. Dianov and A. M. Prokhorov, "Deformation of the resonator of a neodymium glass laser due to a change in the polarizability of excited neodymium ions," Sov. J. Quantum Electron., vol. 8, pp. 275-278, 1978.

[2] U. Hömmerich, H. Eilers, E. Strauss and W. M. Yen, "Optically induced lensing effects in $\mathrm{Nd}^{3+}$ doped laser glass measured by photothermal beamdeflection spectroscopy,” Opt. Lett., vol. 17, pp. 213-214, 1992.

[3] R. C. Powell, S. Payne, L. Chase and G. Wilke, "Four-wave mixing of $\mathrm{Nd}^{3+}$ doped crystals and glasses," Phys. Rev. B, vol. 41, pp. 8593-8602, 1990.

[4] S. C. Weaver, S. Payne, "Determination of excited-state polarizabilities of $\mathrm{Cr}^{3+}$ doped materials by degenerate four-wave mixing," Phys. Rev. B, vol. 40, pp. 10727, 1989.

[5] T. Catunda, M. L. Baesso, Y. Messaddeq and M. Aegerter, "Time-resolved Z scan and thermal lens measurements in $\mathrm{Er}^{3+} \operatorname{smf~} \mathrm{Nd}^{3+}$ doped fluoroindate glasses," J. Non-Cryst. Solids, vol. 213\&214, pp. 225-230, 1997.

[6] M. L. Baesso, J. Shen and R. D. Snook, "Mode Mismatched Thermal Lens Determination of temperature coefficient of Optical Path Length in Soda Lime Glass at Different Wavelengths," J. Appl. Phys., vol. 75, pp. 3733, 1994.

[7] S. M. Lima, T. Catunda, R. Lebullenger, A. C. Hernandes, M. L. Baesso, A. C. Bento and L. C. M. Miranda, "Temperature dependence of thermo-optical properties of fluoride glasses determined by thermal lens spectrometry," Phys. Rev. B, vol. 60, pp. 15173-15178, 1999.

[8] A. A. Andrade, E.Tenório, T. Catunda, M. L. Baesso, A. Cassanho and H. P. Jenssen, "Discrimination between electronic and thermal contributions to the nonlinear refractive index of SrAlF5:Cr ${ }^{3+}$, J. Opt. Soc. Am B., vol. 16, pp. 395-400, 1999.

[9] A. A. Andrade, T. Catunda, R. Lebullenger, A. C. Hernandes and M. L. Baesso, "Time-resolved study of thermal and electronic nonlinearities in $\mathrm{Nd}^{3+}$ doped fluoride glasses,” Electron. Lett., vol. 38, pp. 117-119, 1998. 
[10] L. C. Oliveira, S. C. Zílio, "Single-beam time-resolved Z-scan measurements of slow absorbers," Appl. Phys. Lett., vol. 65, pp. 1-3, 1994.

[11] L. C. Oliveira, T. Catunda and S. C. Zílio, "Saturation effects in Z-scan measurements," Jnp. J. appl. Phys., vol. 35, pp. 25-28, 1996.

[12] V. Pilla, P. R. Impinnisi and T. Catunda, "Measurement of Saturation Intensity in Ion Doped Solids by Transient Nonlinear Refraction," Appl. Phys. Lett., vol. 70, pp. 817, 1997.

[13] M. L. Baesso, J. Shen and R. D. Snook, "Time-resolved thermal lens measurement of thermal diffusivity of soda-lime glass," Chem. Phys. Lett., vol. 197, pp. 255, 1992.

[14] M. Sheik-Bahae, A. A. Said and E. W. V. Stryland, "High sensitivity single beam n 2 measuremente," Opt. Lett., vol. 14, pp. 955-957, 1989.

[15] M. L. Baesso, A. C. Bento, A. R. Duarte, A. M. Neto, L. C. Miranda, J. A. Sampaio, T. Catunda, S. Gama, and F. C. G. Gandra, "Nd2O3 doped low silica calcium aluminosilicate glasses: thermomechanical properties," $J$. Appl. Phys., vol. 85, pp. 8112-8118, 1999.

[16] M. L. Baesso, A. C. Bento, A. A. Andrade, T. Catunda, E. Pecoraro, L. A. O. Nunes, J. A. Sampaio and S. Gama, "Absolute thermal lens to determine fluorescence quantum efficiency and concentration quenching of solids," Phys. Rev. B., vol. 57, pp. 10545-10549, 1998.

[17] M. L. Baesso, J. R. D. Pereira, A. C. Bento, A. J. Palangana, A. M. Mansanares, L. R. Evangelista, "Thermal lens spectrometry to study complex fluids," Braz. J. of Phys., vol. 28, pp. 359-368, 1998.

[18] S. M. Lima, T. Catunda, M. L., Baesso, L. D. Vila, Y. Messaddeq, E.B. Stucchi and S. J. L. Ribeiro, "Thermal-optical properties of Ga:La:S glasses measured by thermal lens technique," J. Non-Cryst. Solids, vol. 247, pp. 222226, 1999.

[19] S. M. Lima, J. A. Sampaio, T. Catunda, R. Lebulleger, A. C. Hernandes, M. L. Baesso, A. C. Bento and F. C. G. Gandra, "Time-resolved thermal lens measurements of thermo-optical properties of fluoride glasses," J. Non-Cryst. Solids, vol. 256\&257, pp. 337-342, 1999.

[20] J. R. D. Pereira, A. M. mansanares, A. J. Palangana, M. L. Baesso, "Molecular crystals and liquid crystals science and technology section a- 
molecular crystals and liquid crystals," Mol. Cryst. and Liq. Cryst. Science and Tech. Section A - Mol. Cryst. and Liq. Cryst., vol. 332, pp. 3079-3085, 1999.

[21] J. R. D. Pereira, A. J. Palanagan, A. M. Mansanares, E. C. da Silva, A. C. Bento and M. L. Baesso, "Inversion in the change of the refractive index and memory effect near the nematic isotropic phase transition in a lyotropic liquid crystal," Phys. Rev. E, vol. 61, pp. 5410-5413, 2000.

[22] A. A. Andrade, T. Catunda,R. Lebullenger, A. C. Hernander, M. L. Baesso, "Electronic and thermal contributions to the non-linear refractive index of $\mathrm{Nd}^{3+}$ ion-doped fluoride glasses," J. Non-Cryst. Solids, vol. 273, pp. 257265, 2000.

[23] J. A. Sampaio, T. Catunda, A. A. Coelho, S. Gama, A. C. Bento, L. C. M. Miranda, and M. L. Baesso, "Thermo-mechanical and optical properties of calcium aluminosilicate glasses doped with $\mathrm{Er}^{3+}$ and $\mathrm{Yb}^{3+}, "$ J. Non-Cryst. Solids, vol. 273, pp. 239-245, 2000.

[24] S. M. Lima, J.A. Sampaio, T. Catunda, A.C. Bento, L.C.M. Miranda, and M.L. Baesso, "Mode-mismatched thermal lens spectrometry for thermooptical properties measurements in optical glasses: a review," J. Non-Cryst. Solids, vol. 273, pp. 215-227, 2000.

[25] J. H. Rohling, A. M. F. Caldeira, J. R. Pereira, A. M. Medina, A. C. Bento, M. L. Baesso and L. C. M. Miranda, "Thermal lens scanning of the glass transition in polymers," J. Appl. Phys., vol. 89, pp. 2220-2226, 2001.

[26] J. H. Rohling, A. N. Medina, A. C. Bento, J. R. D. Pereira, A. F. Rubira, M. L. Baesso and L. C. M. Miranda, "Differential thermal lens temperature scanning approach to glass transition analysis in polymers: application to polycarbonate," J. Phys. D: Appl. Phys., vol. 34, pp. 407-412, 2001.

[27] J. H. Rohling, A. N. Medina, J. R. D. Pereira, A. F. Rubira, A. C. Bento, L. C. M. Miranda, M. L. Baesso, "Thermal lens versus DSC measurements for glass transition analysis of polymer," Anal. Sci., vol. 17, pp. S103-105, 2001.

[28] A. S. Fontes, A. C. Bento, L. C. M. Miranda, M. L. Baesso, "Thermal lens evaluation of the presence of adulterants in brewed coffee," Anal. Sci., vol. 17, pp. S526-529, 2001. 
[29] J. A. Sampaio, T. Catunda, S. Gama, M. L. Baesso, "Thermal-optical properties of $\mathrm{OH}$-free erbium-doped low silica calcium aluminoilicate glasses measured by thermal lens technique," J. Non-Cryst. Solids, vol. 284, pp. 210216, 2001.

[30] S. M. Lima, J.A. Sampaio, T. Catunda, A.S.S. de Camargo, L.A.O. Nunes, M.L. Baesso, and D.W. Hewak, "Spectroscopy, Thermal and Optical Properties of $\mathrm{Nd}^{3+}$ Doped Chalcogenide Glasses," J. Non-Cryst. Solids, vol. 284, pp. 274-281, 2001.

[31] S. M. Lima, A. A. Andrade, T. Catunda, R. Lebullenger, F. Smektala, Y. Jestin and M. L. Baesso, "Thermal and optical properties of chalcohalide glass," J. Non-Cryst. Solids, vol. 284, pp. 203-209, 2001.

[32] S. M. Lima, A. A. Andrade, R. Lebullenger, A. C. Hernandes, T. Catunda and M. L. Baesso, "Multiwavelength thermal lens determination of fluorescence quantum efficiency of solids: Application to $\mathrm{Nd}^{3+}$ - doped fluoride glass," Appl. Phys. Lett., vol. 78, pp. 3220-3222, 2001.

[33] A. A. Andrade, T. Catunda, R. Lebullenger, A. C. Hernandes and M. L. Baesso, "Thermal lens measurements of fluorescence quantum efficiency in

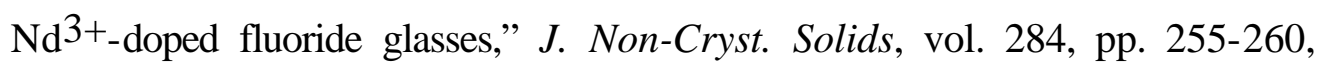
2001.

[34] J. R. D. Pereira, A. M. Mansanares, A. J. Palangana, M. L. Baesso, "Temperature dependence of the refractive index near the reentrant-isotropiccalamitic-nematic phase transition," Phys. Rev. E, vol. 64, 2001.

[35] J. R. D. Pereira, A. M. Mansanares, A. J. Palangana, M. L. Baesso, A. A. Barbosa, P. R. G. Fernandes, "Temperature dependence of the coefficient of electronic polarizability in calamitic nematic liquid crystals," Phys. Rev. E, vol. 64, 2001.

[36] J. A. Sampaio, S. M. Lima, T. Catunda, A. M. Medina, A. C. Bento, M. L. Baesso, "Thermal lens versus DTA measurements for glass transition analysis of fluoride glasses," J. Non-Cryst. Solids, vol. 304, pp. 315-321, 2002.

[37] E. Peliçon, J. H. Rohling, A. N. Medina, A. C. Bento, M. L. Baesso, D. F. de Souza, S. L. Oliveria, J. A. Sampaio, S. M. Lima, L. A. O. Nunes, T. Catunda, "Temperature dependence of fluorescence quantum efficiency of 
optical glasses determined by thermal lens spectrometry," J. Non-Cryst. Solids, vol. 304, pp. 244-250, 2002.

[38] J. H. Rohling, J. Mura, J. R. D. Pereira, A. J. Palangana, A. N. Medina, A. C. Bento, M. L. Bento, L. C. M. Miranda, "Thermal lens temperature scanning for quantitative measurements in complex fluids," Braz. J. Phys., vol. 32, pp. 575-583, 2002.

[39] S. M. Lima, H. Jiao, L. A. O. Nunes and T. Catunda, "Nonlinear refration spectroscopy in resonance with laser lines in solids," Opt. Lett., vol. 27, pp. 845-847, 2002.

[40] S. M. Lima, A. S. S. de Camargo, L. A. O. Nunes, T. Catunda and D. W. Hewak, "Fluorescence quantum efficiency measurements of excitation and nonradiative deexcitation processes of rare earth $4 \mathrm{f}$-states in chalcogenide glasses," Appl. Phys. Lett., vol. 81, pp. 589-591, 2002.

[41] V. Pilla, A. A. Andrade, S. M. Lima, T. Catunda, D. A. Donatti, D. R. Vollet and A. I. Ruiz, "Spectroscopic and thermal characterization in Poly (PPhenylene Vinylene)/Sol-Gel silica sample," Optical Materials (Submetido), 2002.

[42] V. Pilla, T. Catunda, D. T. Balogh, R. M. Faria and S. C. Zilio, "Thermal lensing in poly(vinyl alcohol)/polyaniline blends," J. Pol. Scien. Part B: Pol. Phys., vol. 40, pp. 1949-1956, 2002.

[43] A. A. Andrade, S. M. Lima, V. Plla, J. A. Sampaio, T. Catunda and M. L. Baesso, "Fluorescence quantum efficiency measurements using the thermal lens technique," Review of Scien. Instrum. (Aceito para puplicação), 2002.

[44] A. A. Andrade, T. Catunda, I. Bodnar, J. Mura, M. L. Baesso, "Thermal lens determination of the temperature coefficient of optical path length in optical materials," Review of Scien. Instrum. (Aceito para publicação), 2002.

[45] J. Mura, L. C. M. Miranda, M. L. Baesso, A. C. Bento, A. F. Rubira, "Piezoand pyroelectric photothermal characterization of polymers as a function of temperature," J. Appl. Polym. Sci., vol. 82, pp. 2669-2678, 2001.

[46] W. J. Miniscalco, "Optical and Electronic Properties of Rare Earth ions in Glasses," in Rare Earth doped fiber lasers and amplifiers, D. M.J.F., Ed. Stanford, California: Stanford University, 1993, pp. 19-133. 
[47] D. C. Brown, High-Peak-Power Nd:Glass Laser Systems, vol. 25: Springer Series in Optical Sciences, Springer-Verlag, 1981.

[48] F. Balembois, F. Druon, F. Falcoz, P. Georges and A. Brun, "Performances of Cr:LiSrAlF 6 and $\mathrm{Cr}_{6} \mathrm{LiSrGaF}_{6}$ for continuous-wave diode-pumped Qswitched operation," Opt. Lett., vol. 22, pp. 387-389, 1997.

[49] S. Guy, C. L. Bonner, D. P. Shepherd, D. C. Hanna, A. C. Tropper and B. Ferrand, "High-Inversion Densities in Nd:YAG: Upconversion and Bleaching," IEEE J. Qauntum Electron., vol. 34, pp. 900-909, 1998.

[50] S. A. Payne, G. D. Wilke, L. K. Smith, W. F. Krupke, "Auger upconversion losses in Nd-doped laser glasses," Opt. Commun., vol. 111, pp. 263-268, 1994.

[51] W. Koechner, Solide-State Laser engineering, vol. 01. New York: Spring Verbag, 1988.

[52] T. Catunda, M. L. Baesso, "Glasses and Rare Earth-Doped Glasses for Optical Fibres," Ed. D. Hewak, (INSPEC London, United Kingdom), vol. cp C 14, pp. 180-185, 1998.

[53] T. Catunda, L. A. Cury, "Transverse self-phase modulation in Ruby and $\mathrm{GdAlO}_{3}: \mathrm{Cr}^{3+}$ crystals," J. Opt. Soc. Am. B, vol. 7, pp. 1445-1455, 1990.

[54] H. J. Eichler, P. Gunter and D. W. Pohl, "Laser-Induced Dynamic Gratings," Spring Series in Optical Sciences, vol. 50, 1986.

[55] C. R. Mendonça, B. J. Costa, Y., Messaddeq and S. C. Zílio, "Optical properties of chromium-doped fluoroindate glasses," Phys. Rev. B, vol. 56, pp. 2483-2487, 1997.

[56] R. Powell, S. Payne, "Dispersion Effects in Four-Wave Mixing Measurements of Ion in Solids," Opt. Lett., vol. 15, pp. 1233-1235, 1990.

[57] S. J. Sheldon, L. V. Knight and J. M. Thorne, "Laser-induced thermal lens effect: a new theoretical model," Appl. Opt., vol. 21, pp. 1663-1669, 1982.

[58] R. D. Snook, and R.D. Lowe, Analyst, vol. 120, pp. 2051, 1995.

[59] J. Shen, R.D. Lowe, and R.D. Snook, "A model for cw laser induced modemismatched dual-beam thermal lens spectrometry," Chem. Phys., vol. 165, pp. 385-396, 1992.

[60] S. M. Lima, "Aplicações da Técnica de Lente Térmica em Materiais Ópticos," in Tese de Mestrado. São Carlos: USP, 1999. 
[61] VOLOC, http://www.vloc.com/ncatalog/vloccat.pdf.

[62] L. Arizmendi, J. M. Cabrera and F. Agulló-López, "Material properties and photorefractive bechaviour of BSO family crystals," Int. J. of Optoelectronics, vol. 7, pp. 149-180, 1992.

[63] S. A. Payne, L. K. Smith, R. J. Beach, B. H. T. Chai, J. H. Tassano, L. D. Deloach, W. L. Kway, R. W. Solarz and W. F. Krupke, "Properties of Cr:LiSrAlF 6 crystals for laser operation," Appl. Opt., vol. 33, 1994.

[64] A. Cassanho, H. Jenssen, "LisGaF offers performance edge over LiSAF," Laser Focus World, pp. 169-174, 1997.

[65] B. W. Woods, S. A. Payne, J. E. Marion, R. S. Hugles and L. E. Davis, "Thermomechanical and thermo-optical properties of the $\mathrm{LiCaAlF}_{6}: \mathrm{Cr}^{3+}$ laser material," J. Opt. Soc. Am. B, vol. 8, pp. 970-977, 1991.

[66] A. Rosencwaig, and E.A. Hildum, " $\mathrm{Nd}^{3+}$ fluorescence quantum efficiency measurements with photo-acoustics," Phys. Rev. B, vol. 23, pp. 3301-3307, 1981.

[67] J. N. Demas, and G.A. Crosby, "The Measurment of Photoluminescence Quantum Yields,” J. of Phys. Chem., vol. 75, pp. 991-1024, 1971.

[68] W. M. Yen, and M. D. Levenson, Lasers, Spectroscopy and New Ideas, vol. 54. New York: Springer-Verlag, 1987.

[69] S. E. Stokowski, R. A. Saroyan and M. J. Weber, "Nd-doped laser glass spectrocopic and physical properties," Lawrence Livermore National Laboratory, vol. M-095, November 1981.

[70] J. Fernández, R. Balda and M. A. Arriandiaga, "Spectroscopic and laser properties of $\mathrm{Nd}^{3+}$ in fluoride glasses," Opt. Mat., vol. 4, pp. 91-97, 1994.

[71] E. Pecoraro, "Tese de Doutrorado: Estudo espectroscópico de vidros a base de aluminato de cálcio dopados com $\mathrm{Nd}^{3+}$ e de vidros a base de fluoreto de chumbo dopados com $\operatorname{Pr}^{3+}$ e $\operatorname{Pr}^{3+} / \mathrm{Yb}^{3+}, "$. São Carlos - SP: USP University of São Paulo, 1999.

[72] R. Balda, J. Fernández, A. Mendioroz, J. I. Adam and B. Boulard, "Temperature-dependence concentration quenching of $\mathrm{Nd}^{3+}$ fluorescence in fluoride glasses," J. Phys. Condens. Mater., vol. 6, pp. 913-924, 1994. 
[73] J. C. Murphy, and L.C. Aamodt, "Photoacoustic spectroscopy of luminescent solids: Ruby,” J. Appl. Phys., vol. 48, pp. 3502-3509, 1977.

[74] J. Shen, and R.D. Snook, "Thermal lens measurement of absolute quantum yield using quenched fluorescent samples as references," Chem. Phys. Lett., vol. 155, pp. 583-586, 1989.

[75] M. L. Lesieki, and J.M. Drake, "Use of the thermal lens technique to measure the luminescent quantum yields of dyes in pmma for luminescent solar concentrators," Appl. Opt., vol. 21, pp. 557-560, 1982.

[76] I. M. Thomas, S. A. Payne and G.D. Wilke, "Optical properties and laser demonstration of Nd-doped sol-gel silica glasses," J. Non-Cryst. Solids, vol. 151, pp. 183-194, 1992.

[77] J. A. Muñoz, J.O. Tocho and F. Cussó, "Experimental determination of the luminescent quantum efficiency of the laser emitting level of $\mathrm{Nd}^{3+}$ ions in lithium niobate," Europhysics Lett., vol. 43, pp. 159-169, 1998.

[78] J. H. Brannon, J. Magde, "Absolute quantum yield determination by thermal blooming fluorescein,” J. Phys. Chem., vol. 82, pp. 705-709, 1979.

[79] W. F. Krupke, "Radiative Transition Probabilities Within the $4 \mathrm{f}^{3}$ Ground Configuration of Nd:YAG," IEEE J. Quant. Electron., vol. QE-7, pp. 153$159,1971$.

[80] E. M. Dianov, A.Ya. Karasik, V.B. Neustruev, A.M. Prokhorov, and I.A. Shcherbakov, Sov. Phys. Dokl., vol. 20, pp. 622, 1976.

[81] T. Kushida, and J. E. Geusic, "Optical refrigeration in Nd-doped yttrium aluminum garnet," Phys. Rev. Lett., vol. 21, pp. 1172-1175, 1968.

[82] P. F. Liao, and H. P. Weber, "Fluorescence quenching of the ${ }^{4} F_{3 / 2}$ state in Nd-doped yttrium aluminum garnet (YAG) by multiphonon relaxation," $J$. Appl. Phys., vol. 45, pp. 2931-2934, 1974.

[83] G. J. Linford, R. A. Saroyan, J. B. Trenholme and M. J. Weber, "Measurements and Modeling of Gain Coefficientes for Neodymium Laser Glasses," IEEE J. Quant. Electron., vol. QE-15, pp. 510-523, 1979.

[84] W. F. Krupke, "Induced-Emission Cross Sections in Neodymium Laser Glasses,” IEEE J. Quant. Electron., vol. QE-10, pp. 450-457, 1974. 
[85] J. A. Caird, A. J. Ramponi and P. R. Staver, "Quantum effciency and excitedstate relaxation dynamics in neodymium-doped phosphate laser glasses," $J$. Opt. Soc. Am. B, vol. 8, pp. 1391-1403, 1991.

[86] A. J. Ramponi, and J. A. Caird, "Fluorescence quantum efficiency and optical heating effciency in laser crystals and glasses by laser calorimetry," J. Appl. Phys., vol. 63, pp. 5476-5484, 1998.

[87] R. C. Powell, D. P. Neikirk and D. Sardar, "Radiationless decay processes of $\mathrm{Nd}^{3+}$ ions in solids," J. Opt. Soc. Am., vol. 70, pp. 486-490, 1980.

[88] S. Singh, R. G. Smith and L. G. Van Uitert, "Stimulated-emission cross section and fluorescent quantum efficiency of $\mathrm{Nd}^{3+}$ in yttrium aluminum garnet at room temperature," Phys. Rev. B, vol. 10, pp. 2566-2572, 1974.

[89] F. Batalioto, "Dissertação de Mestrado: Caracterização espectroscópica de vidros fluoroindogalato dopados com $\mathrm{Nd}^{3+}$ e com $\mathrm{Nd}^{3+}$ e $\mathrm{Yb}^{3+}$," . São Carlos: IFSC - USP, 2000, pp. 127.

[90] C. L. Gettinger, A. J. Heeger, J. M. Drake and D. J. Pine, "A photoluminescence study of poly(phenylene vinylene) derivatives: The effect of intrinsic persistence length," J. Chem. Phys., vol. 101, pp. 1673-1678, 1994.

[91] L. Smilowitz, A. Hays, A. J. Heeger, G. Wang and J. E. Bowers, "Timeresolved photoluminescence from poly[2-methoxy, 5-(2'-ethyl-hexyloxy)-pphenylene-vinilene]: Solutions, gels, films, and blends," J. Chem. Phys., vol. 98, pp. 6504-6509, 1993.

[92] M. Sparks, "Optical Distortion by Heated Windows in High-Power Laser Systems," J. Appl. Phys., vol. 42, pp. 5029-5046, 1971.

[93] J. M. Jewell, and I.D. Aggarwal, "Thermal lensing in heavy metal fluoride glasses," J. Non-Cryst. Solids, vol. 142, pp. 260-268, 1992.

[94] J. A. Sampaio, “Tese de doutorado: Preparação e caracterização de vidros aluminato de cálcio com baixa concentração de sílica dopados com $\mathrm{Nd}_{2} \mathrm{O}_{3} \mathrm{e}$ $\mathrm{Er}_{2} \mathrm{O}_{3}$," . São Carlos - SP: USP - University of São Paulo, 2001.

[95] T. Izumitani, T. Yamashita, M. Tokida, K. Miura and H. Tajima, Halide Glasses for Infrared Fiberoptics: Nato Scientific Affairs Division, 1986.

[96] casix, JDS Uniphase Company-http://www.casix.com. 
[97] W. Hayes, Crystals with the Fluorite Structure: Clarendon, Oxford, 1974.

[98] J. L. Doualan, C. Maunier, D. Descamps, J. Landais and R. Moncorgé, "Excited-state absorption and up-conversion losses in the Nd-doped glasses for high-power lasers," Phys. Rev. B, vol. 62, pp. 4459-4463, 2000.

[99] D. L. Dexter, "A theory of Sensitized Luminescence in Solids," J. Chem. Phys., vol. 21, pp. 836-850, 1953.

[100] V. Pilla, "Tese de doutorado: Espectroscopia não linear em cristais fluoretos e polianilina," . São Carlos: Instituto de Física de São Carlos da Universidade de São Paulo - USP, 2001, pp. 213.

[101] Y. Guyot, H. Manaa, J. Y. Rivoire and R. Moncorgé, "Excited-stateabsorption and upconversion studies of $\mathrm{Nd}^{3+}$ - doped single crystals $\mathrm{Y}_{3} \mathrm{Al}_{5} \mathrm{O}_{12}, \mathrm{YLiF}_{4}$, and $\mathrm{LaMgAl}_{11} \mathrm{O}_{19}$," Phys. Rev. B, vol. 51, pp. 784-799, 1995.

[102] V. Pilla, "Dissertação de Mestrado: Medidas de intensidades de saturação por refração não-linear transiente,” . São Carlos: IFSC-USP, 1996.

[103] C. H. Kwak, Y. L. Lee and S. G. Kim, "Analysis of asymmetric Z-scan measurement for large optical nonlinearities in an amorphous $\mathrm{As}_{2} \mathrm{~S}_{3}$ thin film,” J. Opt. Soc. Am. B, vol. 16, pp. 600-604, 1999.

[104] T.-H. Wei, Tzer-Hsiang Huang and Mu-Shil Lin, "Sings of nonlinear refraction in chloroaluminum phthalocyanine solution," Appl. Phys. Lett., vol. 72, pp. 2505-2507, 1998.

[105] M. Sheik-Bahae, A. A. Said, T. Wei, D. Hagan and E. W. V. Etryland, "Sensitive Measurement of Optical Nonlinearities Using a Single Beam," IEEE J. Quantum Electron., vol. 26, pp. 760-769, 1990.

[106] A. A. Andrade, "Dissertação de Mestrado: Estudo do Índice de Refração nãolinear em Cristais e Vidros Fluoretos Dopados," . São Carlos: IFSC - USP, 1998, pp. 99.

[107] H. L. Fragnito, E. Palange, "Medidas de $\mathrm{n}_{2}$ com a técnica de Z-Scan," presented at Escola de Verão Jorge André Swieca de Óptica Quântica e Óptica Não-linear, IFGW- UNICAMP, Campinas, 1994. 
[108] T. Jensen, V. G. Ostroumov, J. P.,Meyn, G. Huber, A. I. Zazumennyi and I. A. Shecherbakov, "Spectrosopic Characterization and Laser Performance of Diode-Laser-Pumped Nd:GdVO," Appl. Phys. B, vol. 58, pp. 373-379, 1994.

[109] R. C. Powell, S. A. Payne, L. L. Chase and G. D. Wilke, "Index of Refraction Change in Optically Pumped Solid Laser Materials," Opt. Lett., vol. 14, pp. $1204-1207,1989$.

[110] E. Tenório, "Dissertação de Mestrado: Estudo de Efeitos de Saturação no Índice de Refração não-liner de Sólidos Dopados," IFSC - USP, São Carlos 1999.

[111] M. Pollnau, P. L. Hardman, M. A. Kern, W. A. Clarkson, and D. C. Hanna, "Upconversion-induced heat generation and thermal lensing in Nd:YLF and Nd:YAG," Phys. Rev. B, vol. 58, pp. 16076-16092, 1998.

[112] Y. F. Chen, C. C. Liao, Y. P. Lan, S. C. Wang, "Determination of the Auger upconversion rate in fiber-coupled diode end-pumped $\mathrm{Nd}: \mathrm{YAG}$ and Nd:YVO 4 crystals," Appl. Phys. B, vol. 70, pp. 487-490, 2000.

[113] M. L. Baesso, A. C. Bento, A. A. Andrade, T. Catunda, J. A. Sampaio and S. Gama, "Neodymium concentration dependence of the thermo-optical properties in low silica calcium aluminate glasses," J. Non-Cryst. Solids, vol. 219, pp. 165-169, 1997.

[114] H. P. Jenssen, S. T. Lai, "Tunable-laser characteristics and spectroscopic properties of SrAlF5:Cr ${ }^{3+}$," J. Opt. Soc. Am. B, vol. 3, pp. 115, 1986.

[115] D. P. Devor, L. G. Deshazer and R. C. Pastor, "Nd:YAG Quantum Efficiency and Related Radiative Properties," IEEE J. Quant. Electron., vol. 25, pp. 1863-1873, 1989.

[116] S. Kuck, L. Fornasiereo, E. Mix, G. Huber, "Excited State Absorption and Stimulated Emission of $\mathrm{Nd}^{3+}$ in Crystals. Part I: $\mathrm{Y}_{3} \mathrm{Al}_{5} \mathrm{O}_{12}, \mathrm{YAlO}_{3}$ and $\mathrm{Y}_{2} \mathrm{O}_{3}$," Appl. Phys. B, vol. 67, pp. 151-156, 1998. 


\section{Apendice A}

\section{A1 Trabalhos publicados.}

1) A. A. Andrade, T. Catunda,R. Lebullenger, A. C. Hernander, M. L. Baesso, "Electronic and thermal contributions to the non-linear refractive index of $\mathrm{Nd}^{3+}$ ion-doped fluoride glasses," J. Non-Cryst. Solids, vol. 273, pp. 257-265, 2000.

2) S. M. Lima, A. A. Andrade, R. Lebullenger, A. C. Hernandes, T. Catunda and M. L. Baesso, "Multiwavelength thermal lens determination of fluorescence quantum efficiency of solids: Application to $\mathrm{Nd}^{3+}$ - doped fluoride glass," Appl. Phys. Lett., vol. 78, pp. 3220-3222, 2001.

3) S. M. Lima, A. A. Andrade, T. Catunda, R. Lebullenger, F. Smektala, Y. Jestin and M. L. Baesso, "Thermal and optical properties of chalcohalide glass," $J$. Non-Cryst. Solids, vol. 284, pp. 203-209, 2001.

4) A. A. Andrade, T. Catunda, R. Lebullenger, A. C. Hernandes and M. L . Baesso, "Thermal lens measurements of fluorescence quantum efficiency in $\mathrm{Nd}^{3+}$-doped fluoride glasses," J. Non-Cryst. Solids, vol. 284, pp. 255-260, 2001.

5) A. A. Andrade, S. M. Lima, V. Pilla, J. A. Sampaio, T. Catunda and M. L. Baesso, "Fluorescence quantum efficiency measurements using the thermal lens technique," Review of Scien. Instrum. (Aceito para puplicação), 2002.

6) A. A. Andrade, T. Catunda, I. Bodnar, J. Mura, M. L. Baesso, "Thermal lens determination of the temperature coefficient of optical path length in optical materials," Review of Scien. Instrum. (Aceito para publicação), 2002. 
7) V. Pilla, A. A. Andrade, S. M. Lima, T. Catunda, D. A. Donatti, D. R. Vollet and A. I. Ruiz, "Spectroscopic and thermal characterization in Poly (P-Phenylene Vinylene)/Sol-Gel silica sample,” Optical Materials (Submetido), 2002 


\section{Apêndice B}

\section{B1 Amostras estudadas}

Durante o desenvolvimento deste trabalho foram realizadas medidas em vidros fluoretos e vidros aluminato de cálcio dopados com $\mathrm{Nd}^{3+}$; em cristais (YAG e YLF ) dopados com $\mathrm{Nd}^{3+}$ e em cristais (LiSGaF e LiSAF ) dopados com $\mathrm{Cr}^{3+}$, crescidos pela equipe do Dr. H. Jenssen [114]. Estudamos também o vidro calcohaleto $\left(40 \% \mathrm{PbI}_{2}-30 \% \mathrm{Sb}_{2} \mathrm{~S}_{3}-30 \% \mathrm{As}_{2} \mathrm{~S}_{3}\right)$, os cristais BTO $\left(\mathrm{Bi}_{12} \mathrm{TiO}_{20}\right)$ e BSO $\left(\mathrm{Bi}_{12} \mathrm{SiO}_{20}\right)$, materiais fotorefrativos pertencentes a família dos silenitas e o filme fino PPV (p-fenileno-vinileno) preparado pelo método "self assemble" (SA) e convertido a $110^{\circ} \mathrm{C}$.

Os vidros fluoretos foram preparados pelo Prof. Dr. R. Lebullenger no laboratório de vidros fluoretos do grupo de materiais do IFSC. O processo de preparação do vidro, após a pesagem dos vários componentes utilizados, consiste em fundir o material em cadinho de platina e verter o vidro líquido em um molde préaquecido. Em seguida, o conjunto "molde-vidro" sofre um recozimento a uma temperatura de $15^{\circ} \mathrm{C}$ abaixo da temperatura de transição vítrea $\left(T_{g}\right)$ da amostra afim de eliminar as tensões internas geradas durante a sua solidificação. Entretanto, no caso dos fluoretos existem muitas condições para a vitrificação ocorrer resultando um vidro de boa qualidade óptica: a água dever ser removida em cada estágio, os materiais de partida devem ser extremamente puros e a contaminação posterior proveniente de cadinhos ou inclusões externas devem ser prevenidas. Para evitar a contaminação por partículas do cadinho utiliza-se cadinho de platina ou de carbono 
vítreo. Ainda assim, pode ocorrer absorção de espécies químicas indesejáveis que serão liberadas durante a fusão. A presença de água e de outras impurezas aniônicas, tais como oxigênio, sulfatos e carbonatos podem influenciar drasticamente a formação vítrea ou provocar absorções indesejáveis no infravermelho. A produção de vidros fluoretos de qualidade óptica requer assim materiais de partida de alto grau de pureza, com fundição realizada em atmosfera seca e limpa.

Os vidros aluminato de cálcio foram preparados por J. Sampaio [94], com a colaboração do Prof. M. Baesso e Prof. S. Gama nos laboratórios do IFGWUNICAMP. Estes vidros foram preparados à vácuo. Neste processo a diminuição da influência da água na estrutura do vidro obtido faz com que este material apresente maior transmissão na região do infravermelho. Este comportamento indica que este vidro é um candidato para aplicações em sistemas ópticos nesta região espectral, tais como janela óptica, filtro de absorção óptica e como meio ativo para laseres de estado sólido, este último, através da introdução de íons de terras raras em sua composição. No caso dos vidros dopados com terras raras é essencial que o vidro base apresente absorção óptica muito baixa na região de comprimentos de onda onde haverá a emissão do laser, obtendo-se uma melhor eficiência no processo de conversão de luz para luz reduzindo assim o efeito de lente térmica ou seja, a interferência dos efeitos térmicos durante a operação do laser.

Na Figura B 1, apresentamos o espectro de absorção do vidro Fluorozirconato (ZBLAN) e do vidro aluminato (LSCAS) e na Tabela B 1 temos a composição dos vidros estudados neste trabalho. 


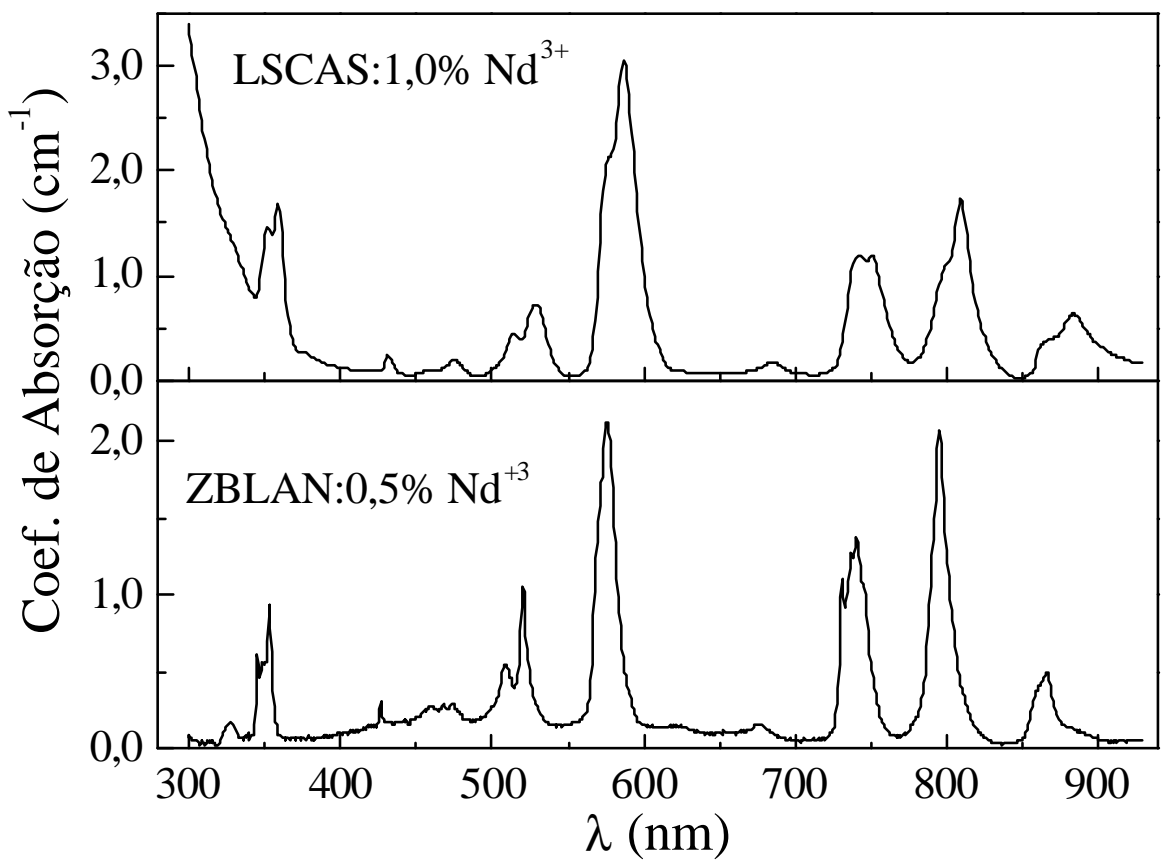

Figura B 1 - Espectro de absorção dos vidros fluorozirconato (ZBLAN) e aluminato (LSCAS).

\begin{tabular}{|c|c|c|c|c|c|c|c|}
\hline \multirow{3}{*}{$\begin{array}{l}\text { Amostra } \\
\text { Aluminato } \\
(L S C A S)\end{array}$} & \multicolumn{7}{|c|}{ Composição $\left(\% N d^{3+}\right)$} \\
\hline & $\mathrm{AlO}_{3}$ & $\mathrm{CaO}$ & $\mathrm{SiO}_{2}$ & $\mathrm{MgO}$ & $\mathrm{Nd}_{2} \mathrm{O}_{3}$ & & \\
\hline & 41,5 & 47,4 & 7 & 4,1 & $x$ & & \\
\hline \multirow{2}{*}{$\begin{array}{l}\text { Fluoroindato } \\
(\operatorname{InSBZnGdN})\end{array}$} & $G d F_{3}$ & $\operatorname{InF} F_{3}$ & $\mathrm{SrF}_{2}$ & $B a F_{2}$ & $Z n F_{2}$ & $N a F$ & $\mathrm{NdF}_{3}$ \\
\hline & 2 & 40 & 20 & 16 & 20 & 2 & 1 \\
\hline \multirow{2}{*}{$\begin{array}{l}\text { Fluorozirconato } \\
(Z B L A N)\end{array}$} & $\mathrm{ZrF}_{4}$ & $\mathrm{BaF}_{2}$ & $\mathrm{LaF}_{3}$ & $\mathrm{AlF}_{3}$ & $N a F$ & $\mathrm{NdF}_{3}$ & \\
\hline & 53 & 29 & 4,5 & 3,5 & 10 & $x$ & \\
\hline \multirow{2}{*}{$\begin{array}{l}\text { Fluoroindato } \\
(P G I Z C a)\end{array}$} & $\mathrm{PbF}_{2}$ & $\mathrm{GaF}_{3}$ & $\mathrm{CaF}_{2}$ & $\operatorname{InF}_{3}$ & $Z n F_{2}$ & $N d F_{3}$ & \\
\hline & 30 & 20 & 20 & 15 & 15 & $x$ & \\
\hline \multirow{2}{*}{$\begin{array}{l}\text { Fluoroaluminato } \\
\qquad(Y A B C)\end{array}$} & $Y F_{3}$ & $\mathrm{AlF}_{3}$ & $\mathrm{BaF}_{2}$ & $\mathrm{CaF}_{2}$ & $\mathrm{NdF}_{3}$ & & \\
\hline & 20 & 40 & 20 & 20 & $x$ & & \\
\hline
\end{tabular}

Tabela B 1- Tabela com a composição dos vidros estudados neste trabalho. Para o aluminato $x=0,5 ; 1,0 ; 2,0 ; 2,5 ; 3,0 ; 4,0$ e 4,5 mol \%. Para o ZBLAN $x=0,5 ; 1,0 ; 2,0 e$ 3,0 mol\%. Para o PGIZCa $x=0,5 ; 1,0 ; 2,0$, e 3,0 mol \%. Para o YABC $x=1,0$ e 2,0 mol \%. 
Os vidros calcohaletos $\quad\left(40 \% \mathrm{PbI}_{2}-30 \% \mathrm{Sb}_{2} \mathrm{~S}_{3}-30 \% \mathrm{As}_{2} \mathrm{~S}_{3}\right)$ são interessantes por apresentarem larga janela de transparência no infravermelho ( 13mm ) e baixa energia de fônon. Estas são característica de vidros com fraca ligação entre seus constituintes (baixo $T_{g}$ ) que apresentam a vantagem de terem baixa condutividade e difusividade térmica.

O filme PPV (p-fenileno-vinileno) foi preparado por meio da técnica "selfassemble" (SA) e submetido a um tratamento térmico a $110^{\circ} \mathrm{C}$ por duas horas sob vácuo. O filme foi preparado pelo Prof. A. Marletta no grupo de polímeros do IFSC.

Os cristais de YAG : $\mathrm{Nd}^{3+}$ utilizados neste trabalho foram de procedências diferentes. Nós utilizamos duas amostras, uma delas, a qual denominaremos de amostra (1), nos foi enviada da Rússia pelo Dr. O. L. Antipov e a outra, denominada (2), foi crescida na Espanha. Estas amostras nos foram fornecidas sem os valores de suas concentrações, o que dificultou muito o nosso trabalho de análise comparativa dos resultados. Para determinar as suas concentrações, fizemos uso da relação $\mathrm{N}=\mathrm{A} / \mathrm{s}$, onde $\mathrm{N}$ é o número de íons por $\mathrm{cm}^{3}, A$ é o coeficiente de absorção e $\sigma$ é a seção de choque de absorção da amostra.

Fizemos experimentos com um laser de $\mathrm{Ar}^{3+}$ em 514,5nm e um laser de $\mathrm{Ti}^{+3}$-safira em $808 \mathrm{~nm}$. Para o cálculo da concentração de $\mathrm{Nd}^{3+}$ nos cristais de YAG, as medidas de absorção em ? 808nm são muito mais convenientes pelo fato do cristal apresentar maior absorção e um pico bem definido neste comprimento de onda tal como mostrado nos espectros de absorção da Figura B 2 e Figura B 3. 


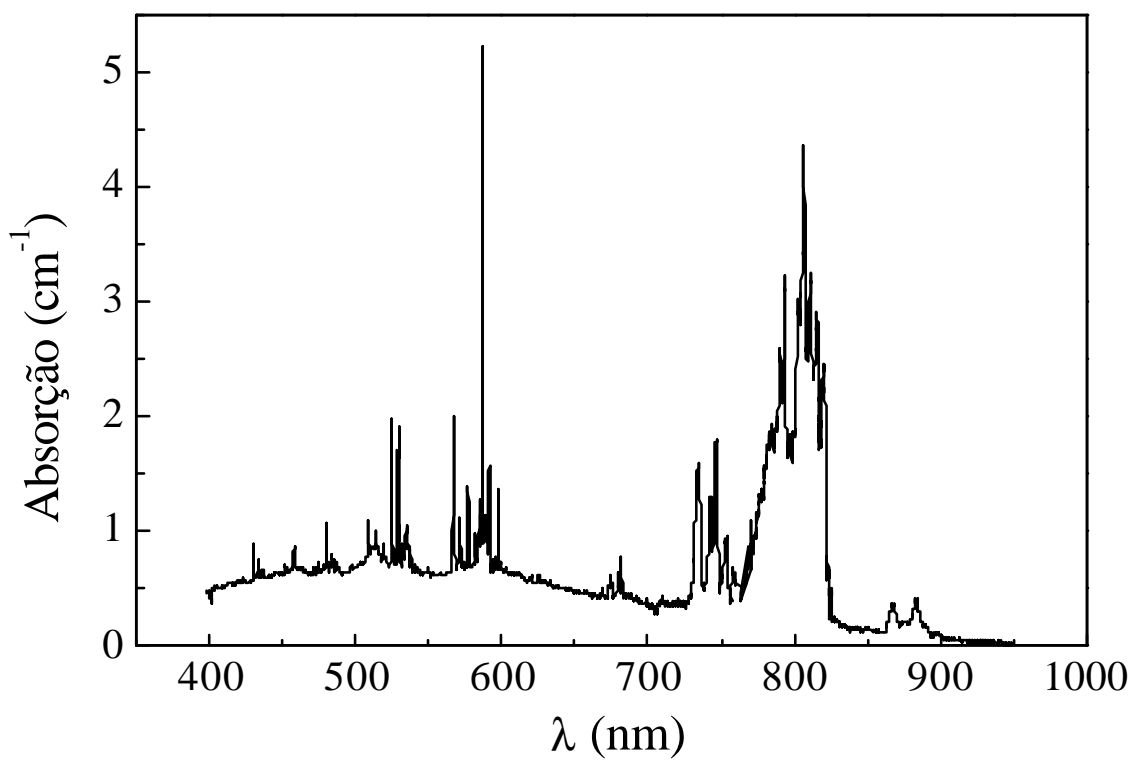

Figura B 2- Espectro de absorção do $Y A G: \mathrm{Nd}^{3+}$, amostra (1). Espessura da amostra $L=1,4 \mathrm{~mm}$.

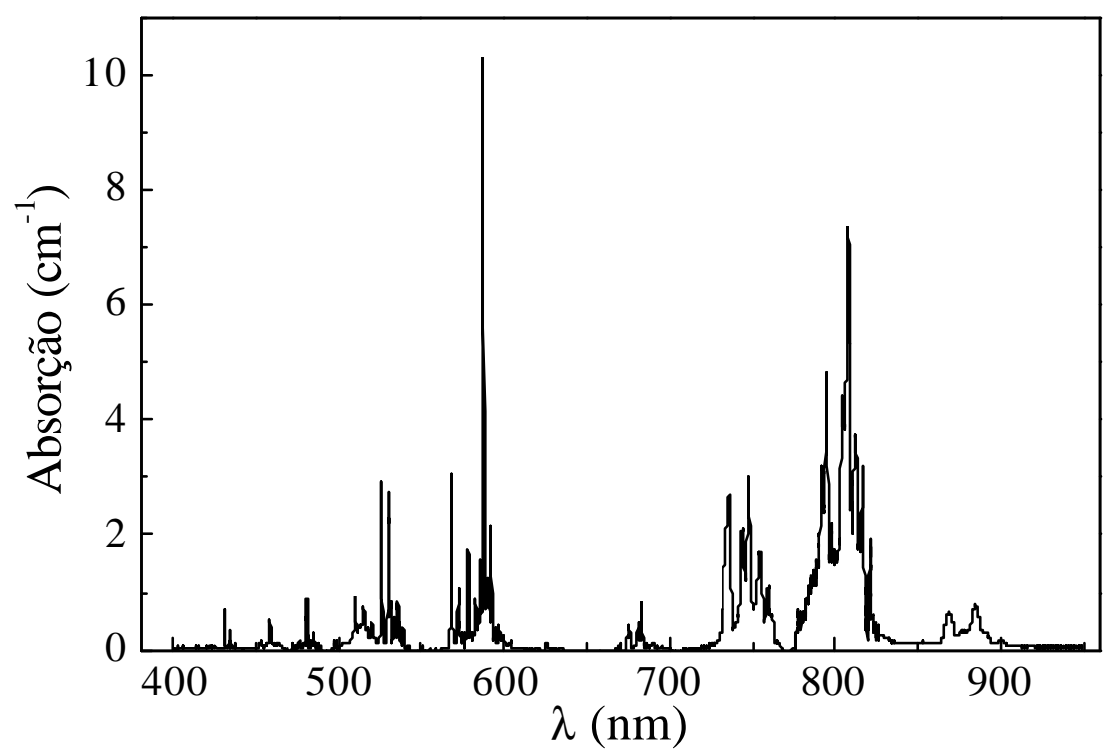

Figura B 3- Espectro de absorção do $Y A G: N d^{3+}$, amostra (2). Espessura da amostra $L=2,1 \mathrm{~mm}$.

Os valores das absorções das amostras, foram obtidos por dois métodos e o melhor resultado foi obtido através das medidas realizadas com o espectrofotômetro Cary Modelo $\mathrm{n}^{0}$ 17. Para a amostra (1) encontramos em 808nm um coeficiente de absorção $\mathrm{A}=4,25 \mathrm{~cm}^{-1}$. Com este valor e o de $\mathrm{s}=0,7 \times 10^{-19} \mathrm{~cm}^{2}$, pudemos estimar 
o seu número de íons $/ \mathrm{cm}^{3} \mathrm{~N}=0,61 \times 10^{20}$ íns $/ \mathrm{cm}^{3}$, o que eqüivale uma concentração de $0,44 \%$ de $\mathrm{Nd}^{3+}$. Da mesma forma para a amostra (2), estimamos $\mathrm{N}=1,03 \times 10^{20}$ íons $/ \mathrm{cm}^{3}$ e uma concentração de $0,75 \%$ de $\mathrm{Nd}^{3+}$.

Utilizando o valor de $N$ encontrado para a amostra (2) e o seu coeficiente de absorção em 514nm extraído do espectro, encontramos a seção de choque de absorção que foi de $\mathrm{s} \sim 0,42 \times 10^{-20} \mathrm{~cm}^{2}$. Este valor está em bom acordo com o encontrado por Devor et al. [115] $\mathrm{s}=0,5 \times 10^{-20} \mathrm{~cm}^{2}$. Para a amostra (1), devido a grande dificuldade em determinar a linha de base, principalmente na região entre $400-700 \mathrm{~nm}$, onde apresenta uma banda larga de absorção, achamos conveniente utilizar o valor da seção de choque encontrado pela amostra (2), $\mathrm{s}=0,42 \times 10^{-20} \mathrm{~cm}^{2}$, que como sabemos independe da concentração de $\mathrm{Nd}^{3+}$, e o valor de $\mathrm{N}=0,61 \times 10^{20}$ íns $/ \mathrm{cm}^{3}$, para estimar o seu coeficiente de absorção em $514 \mathrm{~nm}$, que foi de $0,26 \mathrm{~cm}^{-1}$. Este valor é coerente ao encontrado, quando descontamos a banda de absorção da matriz no intervalo de interesse (430 620). A banda larga de absorção que aparece na amostra (1), certamente deve-se a algum tipo de impureza, já que quando comparado com a amostra (2), não se observa esta absorção.

Para efeito de comparação, determinamos o coeficiente de absorção utilizando a Lei de Lambert-Beer. De acordo com essa lei podemos prever um erro que varia de acordo com a expressão ? $\mathrm{T}=(1-\mathrm{R})^{2} ?(\mathrm{AL}) \exp [-\mathrm{AL}]$, ou seja, ? A $=(1 / \mathrm{L})$ ? T/T . O erro na medida do coeficiente de absorção (? ? A é dado pelo produto do erro relativo na medida da transmitância $(\Delta \mathrm{T} / \mathrm{T})$ vezes o fator $1 / L$. Supondo, por exemplo, ? $\mathrm{T} / \mathrm{T}=0,03$ (ou 3\%) e $\mathrm{L}=1,4 \mathrm{~mm}$ (amostra 1), temos 
? A $=0,21 \mathrm{~cm}^{-1}$ que é um valor alto quando comparado com o coeficiente de absorção em $514 \mathrm{~nm}$, que é menor do que $0,6 \mathrm{~cm}^{-1}$. Por este motivo, em nossos cálculos anteriores utilizamos o coeficiente de absorção obtido através do espectrofotômetro Cary. Para efeito de comparação, na Tabela B 2 encontram-se listados os valores das absorções obtidos pelos dois métodos (em 514 e 808nm) onde podemos comparar as discrepâncias.

\begin{tabular}{ccccc}
\hline \hline Amostra & $\lambda$ & $\begin{array}{c}\text { Espectofotômetro } \\
A\left(\mathrm{~cm}^{-1}\right)\end{array}$ & $\begin{array}{c}\text { Laser } \\
\left(\mathrm{cm}^{-1}\right)\end{array}$ & $\begin{array}{c}N \\
\left(10^{20} \mathrm{~cm}^{-3}\right)\end{array}$ \\
\hline \hline \multirow{2}{*}{$(\mathbf{1})$} & 808 & 4,25 & 3,98 & 0,61 \\
& 514 & 0,26 & 0,52 & \\
$(2)$ & 808 & 7,15 & 7,20 & 1,03 \\
& 514 & 0,43 & 0,60 & \\
\hline
\end{tabular}

Tabela B 2 - Valores do coeficiente de absorção para o YAG em 514 e 808nm.

A Tabela B 3, apresenta valores de coeficientes de absorção e seção de choque de absorção encontrados na literatura para amostras de YAG dopados com diferentes concentrações de $\mathrm{Nd}^{3+}$. Como podemos observar, em 514nm onde o coeficiente de absorção é menor, há uma certa discrepância entre os valores apresentados. Isto certamente deve-se ao fato de que, em $514 \mathrm{~nm}$ a absorção é menor e o pico não é bem definido. 


\begin{tabular}{cccccc}
\hline \hline$\lambda$ & $\% N d^{3+}$ & $\begin{array}{c}A \\
\left(\mathrm{~cm}^{-1}\right)\end{array}$ & $\begin{array}{c}\sigma \\
\left(10^{-19} \mathrm{~cm}^{2}\right)\end{array}$ & $\begin{array}{c}N \\
\left(10^{20} \mathrm{~cm}^{-3}\right)\end{array}$ & Ref. \\
\hline \hline 808 & 1,1 & 11 & 0,70 & 1,57 & Jensen[108] \\
808 & & & 0,70 & & Kuck[116] \\
808 & 1,7 & 14,7 & 0,62 & 2,37 & Guy[49] \\
808 & 3,3 & 28,6 & 0,62 & 4,61 & Guy[49] \\
\hline 514 & 0,7 & 0,47 & 0,050 & 0,97 & Devor[115] \\
514 & & 0,691 & 0,14 & 0,50 & Powell[109] \\
514 & 0,98 & 0,43 & 0,030 & 1,35 & Singh[88] \\
\hline
\end{tabular}

Tabela B 3 - Tabela com os valores de absorção e de seção de choque encontrados na literatura para amostras de YAG com diferentes concentrações e nos comprimentos de onda 514 e $808 \mathrm{~nm}$. 


\section{Apêndice C}

\section{C1 Fator de correção}

Em qualquer medida experimental usando um "chopper" a resolução temporal está limitada ao tempo de subida e descida, ou seja, o tempo que a pá do chopper demora para interromper o feixe de luz. Isto provoca um arredondamento do sinal tal como mostra a Figura C 1 que compara o caso de um feixe com diâmetro grande (partes a e c) com o caso de diâmetro pequeno (partes b e d). Neste apêndice vou comentar como este tempo pode ser minimizado e como corrigir seu efeito nos cálculos.

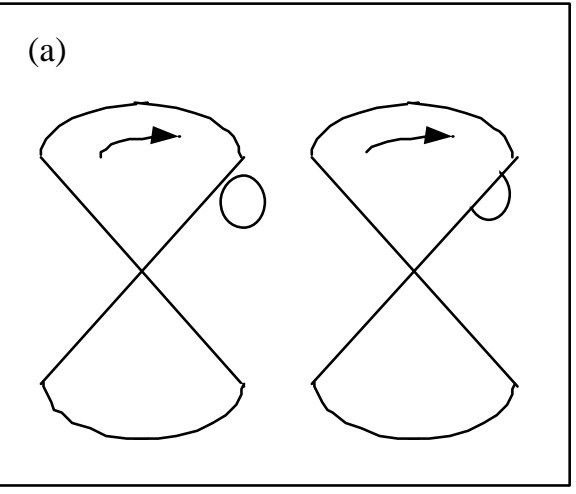

(c)

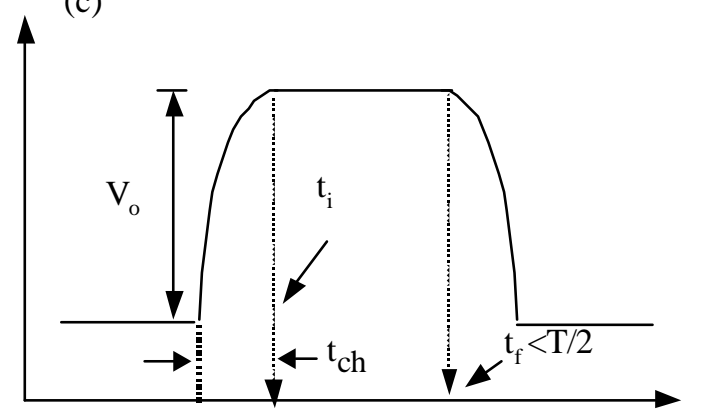

Tempo (b)

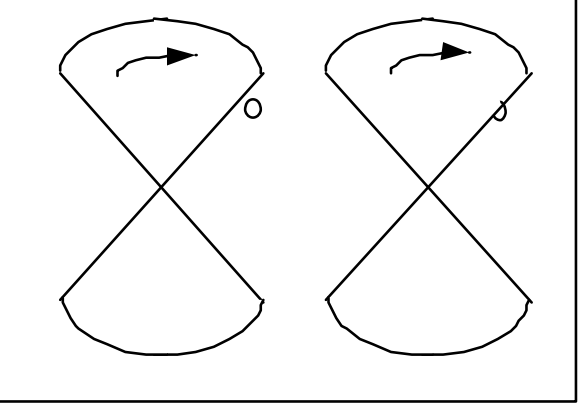

(d)

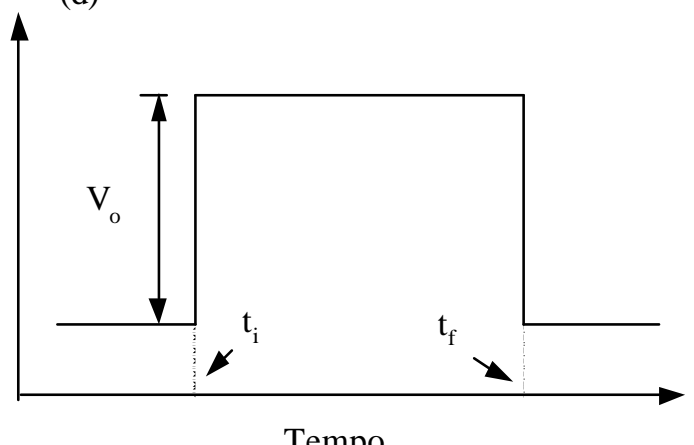

Tempo

Figura C 1 - A Figura (a) e (c) onde há a deformação na forma do sinal. A Figura $(b) e$ (d) apresenta um caso ideal, sem deformação do sinal. 
Este tempo é dado por $\mathrm{t}_{\mathrm{ch}}=\mathrm{v} / 2 \mathrm{w}$, onde $2 \mathrm{w}$ é o diâmetro do feixe do laser, $\mathrm{v}=$ ? R é a velocidade linear da pá do "chopper" na posição do centro do feixe a uma distância $\mathrm{R}$ do centro, e ? a velocidade angular da pá (do disco do "chopper"). Se o disco tem n aberturas, a freqüência do sinal é $f=2 \mathrm{pp} / ?$, logo:

$$
\mathrm{t}_{\mathrm{ch}}=\frac{\mathrm{w}}{\mathrm{pR}} \frac{\mathrm{n}}{\mathrm{f}}
$$

onde podemos observar que $\mathrm{t}_{\mathrm{ch}} \mathrm{e} \mathrm{W}$ são grandezas diretamente proporcionais.

$\mathrm{Na}$ maioria dos nossos experimentos usamos o "chopper" com $n=6$ e $\mathrm{R}=45 \mathrm{~mm}$. Sendo o raio do feixe do laser $(w)$ na saída do laser igual a $750 \mu \mathrm{s}$ e usando uma frequiência de $826 \mathrm{~Hz}$ (o valor mais alto utilizado) da Eq. (C 1) temos um $\mathrm{t}_{\mathrm{ch}}=39 \mu \mathrm{s}$. Este valor representa uma resolução temporal muito baixa em alguns experimentos, por exemplo no caso do $\mathrm{SrAlF}_{5}: \mathrm{Cr}^{3+}$, onde o tempo de vida $\mathrm{t}_{0} \sim 90 \mu \mathrm{s}$. Para obter um bom sinal da evolução temporal de ?n(t) é preciso ter $\mathrm{t}_{\mathrm{ch}}<<\mathrm{t}_{0}$. Isto pode ser feito diminuindo o valor de $\mathrm{w}$ na posição da pá do chopper através de um par de lentes de distância focal curta. Através de experimentos de Z scan sabemos que estas lentes focalizam o feixe com $\mathrm{w} \sim 17 \mu \mathrm{m}, 44$ vezes menor que o valor original, o que nos dá então $\mathrm{t}_{\mathrm{ch}} \sim 1 \mu s$.

Consideraremos agora o cálculo da amplitude ? V do sinal quando a medida é feita em $t_{i}$ e $t_{f}$ (vide Figura C 1). Supondo-se que o sinal é uma exponencial crescente com a amplitude ? $\mathrm{V}$ e tempo $\tau$ temos: 


$$
\begin{aligned}
& \mathrm{V}\left(\mathrm{t}_{\mathrm{f}}\right)=\mathrm{V}_{0}+? \mathrm{~V}\left(1-\exp \left[-\mathrm{t}_{\mathrm{f}} / \mathrm{t}\right]\right) \\
& \mathrm{V}\left(\mathrm{t}_{\mathrm{i}}\right)=\mathrm{V}_{0}+? \mathrm{~V}\left(1-\exp \left[-\mathrm{t}_{\mathrm{i}} / \mathrm{t}\right]\right)
\end{aligned}
$$

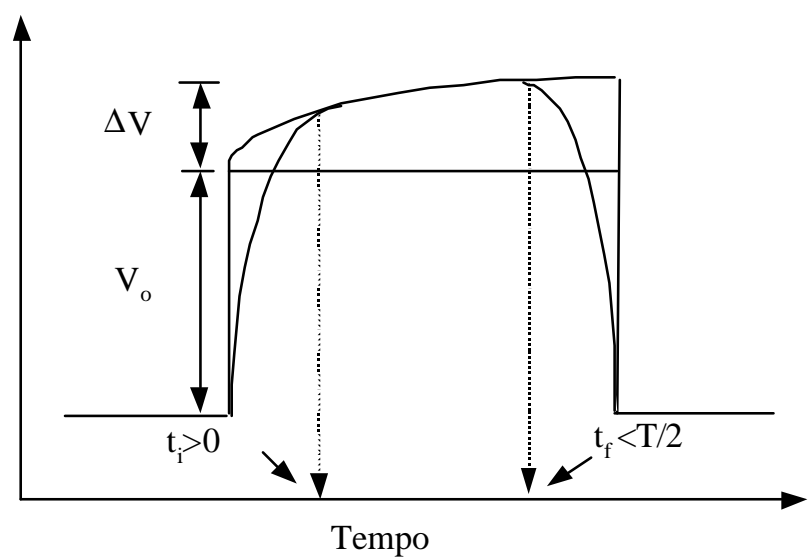

Figura C 2 - Forma do sinal com uma deformação devido ao tempo de abertura do chopper.

Uma vez que no $\mathrm{Z}$-scan trabalha-se com amplitudes pequenas ? $\mathrm{V}<<\mathrm{V}_{0}$, logo de (C 2) e (C 3), temos:

$$
\frac{\mathrm{V}\left(\mathrm{t}_{\mathrm{f}}\right)}{\mathrm{V}\left(\mathrm{t}_{\mathrm{i}}\right)} \approx 1+\frac{\Delta \mathrm{V}}{\mathrm{V}_{0}}-\frac{\Delta \mathrm{V}}{\mathrm{V}_{0}} \exp \left[-\mathrm{t}_{\mathrm{f}} / \tau\right]-\frac{\Delta \mathrm{V}}{\mathrm{V}_{0}}+\frac{\Delta \mathrm{V}}{\mathrm{V}_{0}} \exp \left[-\mathrm{t}_{\mathrm{i}} / \tau\right]-\cdots
$$

portanto teremos:

$$
\frac{\Delta \mathrm{V}}{\mathrm{V}_{0}} \approx \frac{\frac{\mathrm{V}\left(\mathrm{t}_{\mathrm{f}}\right)}{\mathrm{V}\left(\mathrm{t}_{\mathrm{i}}\right)}-1}{\left(\exp \left[-\mathrm{t}_{\mathrm{i}} / \tau\right]-\exp \left[-\mathrm{t}_{\mathrm{f}} / \tau\right]\right)}
$$

onde

$$
\frac{\mathrm{V}\left(\mathrm{t}_{\mathrm{f}}\right)}{\mathrm{V}\left(\mathrm{t}_{\mathrm{i}}\right)}-1=? \mathrm{~T}
$$

